\title{
Mechanochemical Synthesis of Tripodal Tris[4-(1,2,3-triazol-5- ylidene)methyl]amine Mesoionic Carbene Ligands and Their Complexation With Silver(I)
}

Zhi Zhong Ang, ${ }^{a}$ Shoba Laxmi, ${ }^{a}$ Félix León, ${ }^{a}$ Josephine E. M. Kooij,, ${ }^{a}$ Felipe García, ${ }^{\text {*a Jason }}$ England*a

a Division of Chemistry and Biological Chemistry, School of Physical and Mathematical Sciences, Nanyang Technological University, 21 Nanyang Link, Singapore 637371. Email: fgarcia@ntu.edu.sg; jengland@ntu.edu.sg.

Table of Contents Page

General Methods S2

Synthesis and Characterization S3

Aryl Azides 2b- d

Tris[4-(1-aryl-1,2,3-triazol-5-yl)methyl]amines $\mathbf{3 a - d}$ S4

Salts $[4 a-d]\left(B F_{4}\right)_{3}$ S5

Salts $[\mathbf{4 a}-\mathbf{d}](\mathrm{OTf})_{3}$

Silver Complexes, $\left[\mathrm{Ag}_{3}(\mathbf{1} \mathbf{a}-\mathbf{d})_{2}\right][\mathrm{X}]_{3}\left(\mathrm{X}=\mathrm{OTf}\right.$ and $\left.\mathrm{BF}_{4}\right)$ S9

Synthesis of Cobalt Complexes

NMR Spectra

Solution State vs Ball Milling

Mass Spectrometry

X-Ray Crystallography

Density Functional Theory (DFT) Calculations 


\section{General Methods}

Unless otherwise stated, all reactions were performed in the presence of air. Dichloromethane was distilled over $\mathrm{CaH}_{2}$ prior to use. Aryl azides $\mathbf{2} \mathbf{a}^{[1]}, \mathbf{2} \mathbf{b}^{[2]}$ and $\mathbf{2} \mathbf{d}^{[3]}$ were prepared via published procedures. Compound $\mathbf{2 c}$ is prone to self-decay, so was used in the synthesis of $\mathbf{3 c}$ within 2 days of preparation. Silver complexes were stored in the dark, in an $\operatorname{Ar}$ atmosphere glovebox. ${ }^{1} \mathrm{H},{ }^{19} \mathrm{~F}$, ${ }^{13} \mathrm{C}\left\{{ }^{1} \mathrm{H}\right\}$ and DEPT-135 NMR spectra were recorded on either Bruker Avance or JEOL ECA $400 \mathrm{MHz}$ spectrometers. The chemical shift values are reported in parts per million (ppm) and are referenced against residual protic solvent peaks. Mass spectra were recorded using a JEOLT100LP electrospray ionization mass spectrometer (ESI-MS). Whereas respective ion source desolvating chamber and orifice 1 temperatures of 250 and $80{ }^{\circ} \mathrm{C}$ were used for the neutral compounds 3a-d, ion source desolvating chamber and orifice 1 temperatures of 40 and $35{ }^{\circ} \mathrm{C}$, respectively, were used for the ionic species derived from them. Solid-state reactions were conducted in $10 \mathrm{~mL}$ stainless steel milling jars containing a single $10 \mathrm{~mm}$ stainless steel milling ball. Subsequent to addition of reactants, these jars were placed onto a Retsch Mixer Mill MM400 and subjected to $25 \mathrm{~Hz}$ milling for the times specified below.

Caution: Aryl azides are potentially explosive. Although no safety problems were encountered during our work, precautions against possible explosion should be implemented when handling aryl azides dry or suspended in solution.

\section{Solution Magnetic Susceptibility Measurements}

The solution state molar magnetic susceptibility $\left(\chi_{m}\right)$ of $[\mathrm{Co}(\mathbf{1 a})(\mathrm{MeCN})](\mathrm{OTf})_{2}$ was measured using the Evans' NMR method. ${ }^{[4-7]}$ This involved placement of a coaxial insert containing a solution of complex into a standard $5 \mathrm{~mm} \mathrm{NMR}$ tube containing only solvent. $\mathrm{CD}_{3} \mathrm{CN}$ containing one drop of benzene was used as the solvent throughout. Based upon the chemical shift difference of the benzene internal reference between inner and outer tubes, molar magnetic susceptibility $\left(\chi_{m}, \mathrm{~m}^{3} \mathrm{~mol}^{-1}\right)$ and magnetic moment $\left(\mu_{\text {eff }}\right)$ values were calculated using the following equations: ${ }^{[8]}$

$$
\begin{gathered}
\chi_{m}=\frac{3 \cdot \Delta f}{1000 \cdot f \cdot c} \\
\mu_{e f f}=798 \sqrt{T \cdot \chi_{m}}
\end{gathered}
$$


$\Delta f=$ difference in the chemical shift $(\mathrm{Hz})$ of the internal reference between the inner and outer tubes; $f=$ operating frequency of the NMR spectrometer $(\mathrm{Hz}) ; c=$ concentration of the sample $\left(\mathrm{mol} \mathrm{dm} \mathrm{dm}^{-3}\right) ; T=$ temperature of the measurement $(\mathrm{K})$.

\section{Synthesis and Characterization}<smiles>Nc1cc(C(F)(F)F)ccc1C(F)(F)F</smiles>

1-azido-2,5-bis(trifluoromethyl)benzene (2c): 2,5-bis(trifluoromethyl)aniline (10.2 $\mathrm{mL}, 65.5$ $\mathrm{mmol}$ ) was added to an ice bath cooled round-bottom flask containing $130 \mathrm{~mL}$ of $6 \mathrm{M} \mathrm{HCl}$. After stirring for 5 minutes, 1.2 equiv of $\mathrm{NaNO}_{2}(5.42 \mathrm{~g}, 78.0 \mathrm{mmol})$ dissolved in water $(65 \mathrm{~mL})$ was added dropwise to it. Subsequent to stirring at $0{ }^{\circ} \mathrm{C}$ for $2 \mathrm{~h}$, a solution of 1.5 equiv $\mathrm{NaN}_{3}(6.38 \mathrm{~g}$, $98.3 \mathrm{mmol}$ ) in water $(33 \mathrm{~mL})$ was added dropwise. The resulting reaction mixture was allowed to stir at $0{ }^{\circ} \mathrm{C}$ for a further $2 \mathrm{~h}$, before allowing it to warm to room temperature. Next, the aqueous layer was extracted thrice with hexane*. The combined organic layers were washed with brine, dried over $\mathrm{Na}_{2} \mathrm{SO}_{4}$, filtered, and all volatiles removed from the filtrate, using a rotary evaporator, to give the pure $2 \mathrm{c}^{* *}$ an orange-yellow oil $\left.(12.3 \mathrm{~g}, 74 \%) .{ }^{1} \mathrm{H} \mathrm{NMR}_{(\mathrm{CDCl}}, 400 \mathrm{MHz}\right): \delta 7.48$ (d, $1 \mathrm{H}, J=8 \mathrm{~Hz}), 7.51(\mathrm{~s}, 1 \mathrm{H}) 7.78(\mathrm{~d}, 1 \mathrm{H}, J=8 \mathrm{~Hz}) \cdot{ }^{13} \mathrm{C}\left\{{ }^{1} \mathrm{H}\right\} \quad \mathrm{NMR}\left(\mathrm{CDCl}_{3}, 100 \mathrm{MHz}\right): \delta 116.7(\mathrm{q}, J=4$ $\mathrm{Hz}$, aryl CH), $121.3\left(\mathrm{q}, J=4 \mathrm{~Hz}\right.$, aryl CH), $122.5\left(\mathrm{q}, J=273 \mathrm{~Hz}, \mathrm{CF}_{3}\right), 123.1\left(\mathrm{q}, J=273 \mathrm{~Hz}, \mathrm{CF}_{3}\right), 124.5$ $\left(q, J=32 \mathrm{~Hz}, \mathrm{C}\left(\mathrm{CF}_{3}\right)\right), 128.6\left(\mathrm{q}, J=5 \mathrm{~Hz}\right.$, aryl CH), $135.7\left(\mathrm{q}, J=33 \mathrm{~Hz}, \mathrm{C}\left(\mathrm{CF}_{3}\right)\right), 140.1\left(\mathrm{~s}, \mathrm{C}\left(\mathrm{N}_{3}\right)\right) .{ }^{19} \mathrm{~F}$ $\operatorname{NMR}\left(\mathrm{CDCl}_{3}, 376 \mathrm{MHz}\right): \delta-62.9,-63.9$.

*Extraction with hexane yields significant amounts of white precipitate, which is primarily the corresponding triazene. It is removed during the subsequent filtration step.

${ }^{* *} 2 c$ is unstable with respect to formation of the 1,3-[2,5-bis(trifluoromethyl)phenyl]triazene and, prior to use, it may prove necessary to remove white solids by filtration. To minimize decomposition, it should be stored in a freezer. 
General synthetic procedure for the tris[4-(1-aryl-1,2,3-triazol-5-yl)methyl]amines, 3a-d

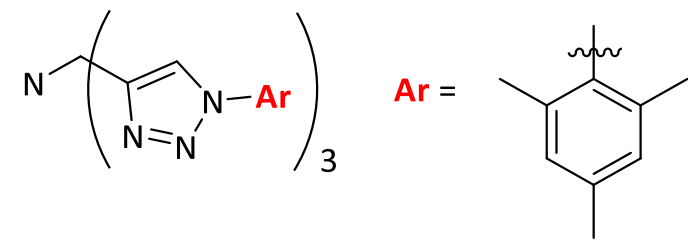

$3 a$<smiles>CC(C)(C)c1ccc(C(F)(F)F)cc1</smiles>

3b<smiles>CC(C)(C)c1ccc(C(F)(F)F)cc1C(F)(F)F</smiles>

3c<smiles>CN(C)c1cc(C(F)(F)F)cc(C(F)(F)F)c1</smiles>

3d

To a stirring solution of $\mathrm{Cu}(\mathrm{OAc})_{2}$ (0.022 equiv) in acetonitrile $(80-130 \mathrm{~mL})$, under an $\mathrm{Ar}$ atmosphere, 1.8/1.9* equiv of 2 (5.00 g), 1 equiv tripropargylamine, and an aqueous solution (5 $\mathrm{mL}$ ) of sodium ascorbate (0.022 equiv) were added. After stirring at room temperature for 30 min, the mixture was heated at $50^{\circ} \mathrm{C}$ for 5 hours. Subsequently, a second batch of $1.8 / 1.9^{*}$ equiv 2 (5.00 g) was added and heating was continued overnight**. Upon cooling, all volatiles were removed from the reaction mixture. The resulting residue was extracted by vigorously stirring for $1 \mathrm{~h}$ with a mixture comprised of an equal volume of dichloromethane (100-170 mL,) and 28 $-30 \% \mathrm{NH}_{4} \mathrm{OH}$ solution (100 - $170 \mathrm{~mL}$ ). The organic layer was separated, and the aqueous layer was extracted twice more using dichloromethane. The organic layers were combined, washed once with sat. $\mathrm{NH}_{4} \mathrm{OH}$ solution $\left(50 \mathrm{~mL}\right.$ ) and then twice with water, dried over $\mathrm{Na}_{2} \mathrm{SO}_{4}$, and filtered. The filtrate was reduced to dryness in vacuo and the solid obtained triturated with a minimal amount of cold diethyl ether. This gave the product as a white/off-white powder, which was isolated by filtration and dried under vacuum.

* $2 a$ and $2 b$ : 1.8 equiv; $2 c$ and $2 d$ : 1.9 equiv.

** in the case of $\mathbf{2 c}$, heating for 2 days was required

3a was obtained as a white solid $(9.74 \mathrm{~g}, 92 \%) .{ }^{1} \mathrm{H}$ NMR $\left(\mathrm{CDCl}_{3}, 400 \mathrm{MHz}\right): \delta 1.99(\mathrm{~s}, 18 \mathrm{H}), 2.35$ (s, 9H), 3.95 (s, 6H), $6.99(\mathrm{~s}, 6 \mathrm{H}), 7.95(\mathrm{~s}, 3 \mathrm{H}) .{ }^{13} \mathrm{C} \mathrm{NMR}\left(\mathrm{CDCl}_{3}, 100 \mathrm{MHz}\right): \delta 17.4\left(\mathrm{o}-\mathrm{CH}_{3}\right), 21.2$ (p$\left.\mathrm{CH}_{3}\right), 47.0\left(\mathrm{CH}_{2}\right), 126.2(\operatorname{Trz} \mathrm{CH}), 129.1(\mathrm{~m}-\mathrm{CH}), 133.6\left(\mathrm{C}\left(\mathrm{p}-\mathrm{CH}_{3}\right)\right), 135.1\left(\mathrm{C}\left(\mathrm{o}-\mathrm{CH}_{3}\right)\right), 140.0(\mathrm{C}), 143.4$ (C). ESI-MS (523 K): $m / z 615.38[3 a+H]^{+}$(calculated = 615.36).

3b was obtained as a white solid (8.32 g, $81 \%) .{ }^{1} \mathrm{H}$ NMR $\left(\mathrm{CDCl}_{3}, 400 \mathrm{MHz}\right): \delta 3.98(\mathrm{~s}, 6 \mathrm{H}), 7.80$ (d, $J=9 \mathrm{~Hz}, 6 \mathrm{H}), 7.93(\mathrm{~d}, J=8 \mathrm{~Hz}, 6 \mathrm{H}), 8.33(\mathrm{~s}, 3 \mathrm{H}) .{ }^{13} \mathrm{C} \mathrm{NMR}\left(\mathrm{CDCl}_{3}, 100 \mathrm{MHz}\right): \delta 47.5\left(\mathrm{~s}, \mathrm{CH}_{2}\right), 120.4$ (s, o-CH), 122.0 (s, Trz CH), 123.7 (q, J = 270 Hz, CF $)_{3}, 127.2$ (q, J = 4 Hz, m-CH), 130.8 (q, J = $33 \mathrm{~Hz}$, $\left.\mathrm{C}\left(\mathrm{CF}_{3}\right)\right), 139.5$ (s, C), 145.2 (s, C). $\left.{ }^{19} \mathrm{~F} \mathrm{NMR} \mathrm{(CDCl}, 376 \mathrm{MHz}\right): \delta$-62.6. ESI-MS (523 K): $\mathrm{m} / z 693.22$ $[3 \mathbf{b}+\mathrm{H}]^{+}($calculated $=693.18)$. 
3c was obtained as an off-white solid (5.18 g, $79 \%) .{ }^{1} \mathrm{H} \mathrm{NMR}\left(\mathrm{CDCl}_{3}, 400 \mathrm{MHz}\right): \delta 3.99,(\mathrm{~s}, 6 \mathrm{H})$, $7.90(\mathrm{~s}, 3 \mathrm{H}), 7.94$ - $7.99(\mathrm{~m}, 3 \mathrm{H}), 8.01$ - $8.06(\mathrm{~m}, 3 \mathrm{H}), 8.16(\mathrm{~s}, 3 \mathrm{H}) .{ }^{13} \mathrm{C} \mathrm{NMR}\left(\mathrm{CDCl}_{3}, 100 \mathrm{MHz}\right): \delta$ $47.1\left(\mathrm{~s}, \mathrm{CH}_{2}\right), 122.1$ (q, J = 273 Hz, CF $), 122.5$ (q, J = $\left.273 \mathrm{~Hz}, \mathrm{CF}_{3}\right), 126.4$ (q, J = $4 \mathrm{~Hz}$, aryl CH), 126.7 (s, $\operatorname{Trz} \mathrm{CH}), 127.5\left(\mathrm{q}, J=4 \mathrm{~Hz}\right.$, aryl CH), $128.6(\mathrm{q}, J=5 \mathrm{~Hz}, \operatorname{aryl} \mathrm{CH}), 129.5\left(\mathrm{q}, J=33 \mathrm{~Hz}, \mathrm{C}\left(\mathrm{CF}_{3}\right)\right.$ ), $135.6\left(\mathrm{q}, J=34 \mathrm{~Hz}, \mathrm{C}\left(\mathrm{CF}_{3}\right)\right), 135.8$ (s, C), $144.4(\mathrm{~s}, \mathrm{C}) .{ }^{19} \mathrm{~F} \mathrm{NMR}\left(\mathrm{CDCl}_{3}, 376 \mathrm{MHz}\right): \delta$-59.7, -63.4. ESI-MS (523 K): $m / z 897.18[3 c+H]^{+}$(calculated $\left.=897.15\right)$.

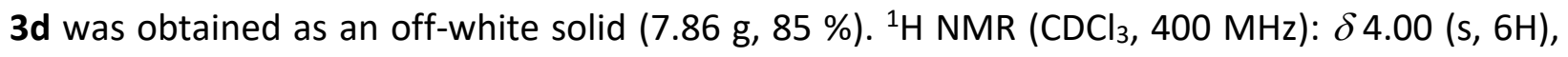
$7.96(\mathrm{~s}, 3 \mathrm{H}), 8.29$ (s, 6H), 8.38 (s, 3H). ${ }^{13} \mathrm{C} \mathrm{NMR}\left(\mathrm{CDCl}_{3}, 100 \mathrm{MHz}\right): \delta 47.4$ (s, $\left.\mathrm{CH}_{2}\right), 120.4$ (s, o- $\left.\mathrm{CH}\right)$, 122.1 (s, Trz CH), 122.2-122.5 (m, p-CH), 122.7 (q, J = $\left.273 \mathrm{~Hz}, \mathrm{CF}_{3}\right), 133.8$ (q, J = $34 \mathrm{~Hz}, \mathrm{C}\left(\mathrm{CF}_{3}\right)$ ), 138.1 (s, C), $145.7(\mathrm{~s}, \mathrm{C}) .{ }^{19} \mathrm{~F} \mathrm{NMR}\left(\mathrm{CDCl}_{3}, 376 \mathrm{MHz}\right): \delta$-63.0. ESI-MS $(523 \mathrm{~K}): \mathrm{m} / \mathrm{z} 897.18[3 \mathbf{d}+\mathrm{H}]^{+}$ (calculated $=897.15)$.

\section{Synthesis of $[4 a-d]\left(B_{4}\right)_{3}$}

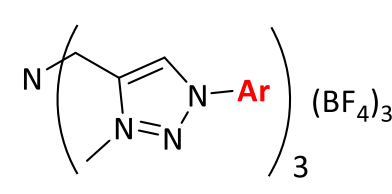

$[4]\left(\mathrm{BF}_{4}\right)_{3}$

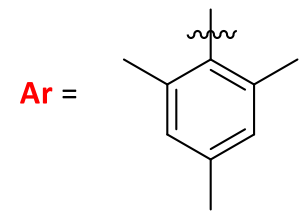

4a<smiles>CC(C)(C)c1ccc(C(F)(F)F)cc1</smiles>

$4 \mathrm{~b}$<smiles>FC(F)(F)c1ccc(C(F)(F)F)c(C(F)(F)F)c1</smiles>

4c<smiles>FC(F)(F)c1cc(C(F)(F)F)cc(C(F)(F)F)c1</smiles>

4d

Solution state. Inside an Ar filled glovebox, a Schlenk tube was charged with 1 equiv of 3 (0.500 g), 4.5 equiv of $\left[\mathrm{Me}_{3} \mathrm{O}\right] \mathrm{BF}_{4}$, and a magnetic stirring bar. Dry dichloromethane $(12 \mathrm{~mL})$ was added to the flask and the resulting mixture stirred for $2 \mathrm{~h}$, at ambient temperatures. Subsequently, the reaction was quenched by addition of methanol $(13 \mathrm{~mL})$, after which it was allowed to stir for 1 hr. Next, all volatiles were removed using a rotary evaporator and the residue was triturated with methanol $(7 \mathrm{~mL})$. The solid obtained was isolated by filtration, washed sequentially with methanol and diethyl ether, and dried in vacuo. It was then washed with $20 \mathrm{~mL}$ portions of water until the $\mathrm{pH}$ of the filtrate reached $\sim 7$. The resulting white solid was collected, dried in vacuo, and recrystallized from acetonitrile solutions layered with diethyl ether. This gave the products $[4 a]\left(B F_{4}\right)_{3},[4 b]\left(B F_{4}\right)_{3}$ and $[4 d]\left(B F_{4}\right)_{3}$ as white/colourless crystals in yields of $34 \%(0.254 \mathrm{~g}), 30 \%$ $(0.216 \mathrm{~g})$ and $33 \%(0.221 \mathrm{~g})$, respectively. Difficulties were experienced in purifying $[4 \mathrm{c}]\left(\mathrm{BF}_{4}\right)_{3}$ due to it having similar solubility properties to the accompanying impurities. 
Solid state. Inside an Ar-filled glovebox, a $10 \mathrm{~mL}$ stainless steel milling jar was charged with 1 equiv 3 ( $0.500 \mathrm{~g}$ ), 3.3 equiv $\left[\mathrm{Me}_{3} \mathrm{O}\right] \mathrm{BF}_{4}$, and a $10 \mathrm{~mm}$ stainless steel ball. The sealed jar was then removed from the glovebox and subjected to $25 \mathrm{~Hz}$ milling for $2 \mathrm{~h}$. Afterwards, all the solids were transferred to a round bottom flask and stirred with ethanol $(7 \mathrm{~mL})$ for $2 \mathrm{hr}$. The solids obtained were collected by filtration and was washed with water until the $\mathrm{pH}$ of the filtrate reached $\sim 7$. To remove remaining by-products, the solid was washed with warm water $\left(60-70{ }^{\circ} \mathrm{C}\right)$ until it was judged to be clean (by ${ }^{1} \mathrm{H}$ NMR spectroscopy), followed by a small amount of cold methanol and, lastly, diethyl ether. Drying under vacuum gave the products $[4 a]\left(B F_{4}\right)_{3},[4 b]\left(B F_{4}\right)_{3}$, $[4 \mathrm{c}]\left(\mathrm{BF}_{4}\right)_{3}$, and $[4 \mathrm{~d}]\left(\mathrm{BF}_{4}\right)_{3}$ as white powders in yields of $60 \%(0.449 \mathrm{~g}), 54 \%(0.389 \mathrm{~g}), 52 \%(0.349$ g) and $60 \%(0.402 \mathrm{~g})$, respectively.

[4a] $\left(\mathrm{BF}_{4}\right)_{3} .{ }^{1} \mathrm{H}$ NMR $\left(\mathrm{CD}_{3} \mathrm{CN}, 400 \mathrm{MHz}\right): \delta 2.05(\mathrm{~s}, 18 \mathrm{H}), 2.38(\mathrm{~s}, 9 \mathrm{H}), 4.27(\mathrm{~s}, 9 \mathrm{H}), 4.36(\mathrm{~s}, 6 \mathrm{H}), 7.17$ $(\mathrm{s}, 6 \mathrm{H}), 8.60$ (s, 3H). $\left.{ }^{13} \mathrm{C} N M R\left(\mathrm{CD}_{3} \mathrm{CN}, 100 \mathrm{MHz}\right): \delta 17.2\left(\mathrm{o}-\mathrm{CH}_{3}\right), 21.1\left(\mathrm{p}-\mathrm{CH}_{3}\right), 39.4\left(\mathrm{~N}_{(\mathrm{CH}}\right)\right), 48.1$ $\left(\mathrm{CH}_{2}\right), 130.5(\mathrm{~m}-\mathrm{CH}), 131.6(\mathrm{Trz} \mathrm{CH}), 132.3\left(\mathrm{C}\left(\mathrm{p}-\mathrm{CH}_{3}\right)\right), 135.7\left(\mathrm{C}\left(\mathrm{o}-\mathrm{CH}_{3}\right)\right), 142.7$ (C), $143.6(\mathrm{C}) .{ }^{19} \mathrm{~F}$ $\operatorname{NMR}\left(\mathrm{CD}_{3} \mathrm{CN}, 376 \mathrm{MHz}\right): \delta$-151.3. ESI-MS $(313 \mathrm{~K}): \mathrm{m} / z 833.45\left\{[4 \mathrm{a}]\left(\mathrm{BF}_{4}\right)_{2}\right\}^{+}$(calculated = 833.43).

[4b] $\left(\mathrm{BF}_{4}\right)_{3} .{ }^{1} \mathrm{H}$ NMR $\left(\mathrm{CD}_{3} \mathrm{CN}, 400 \mathrm{MHz}\right): \delta 4.33(\mathrm{~s}, 9 \mathrm{H}), 4.37(\mathrm{~s}, 6 \mathrm{H}), 8.04(\mathrm{~d}, 6 \mathrm{H}), 8.15(\mathrm{~d}, 6 \mathrm{H}), 9.06$ (s, 3H). ${ }^{13} \mathrm{C}$ NMR $\left(\mathrm{CD}_{3} \mathrm{CN}, 100 \mathrm{MHz}\right): \delta 39.5\left(\mathrm{~s}, \mathrm{~N}\left(\mathrm{CH}_{3}\right)\right), 47.8\left(\mathrm{~s}, \mathrm{CH}_{2}\right), 123.7(\mathrm{~s}, \mathrm{O}-\mathrm{CH}), 124.4(\mathrm{q}, J=$ $\left.271 \mathrm{~Hz}, \mathrm{CF}_{3}\right), 128.5$ (q, J = 4 Hz, m-CH), 128.9 (s, Trz CH), 133.7 (q, J = $\left.33 \mathrm{~Hz}, \mathrm{C}\left(\mathrm{CF}_{3}\right)\right), 138.6$ (s, C), $142.0(\mathrm{~s}, \mathrm{C}) .{ }^{19} \mathrm{~F} \mathrm{NMR}\left(\mathrm{CD}_{3} \mathrm{CN}, 376 \mathrm{MHz}\right): \delta$-63.5, -151.2. ESI-MS $(313 \mathrm{~K}): \mathrm{m} / z 911.33\left\{[4 \mathrm{~b}]\left(\mathrm{BF}_{4}\right)_{2}\right\}^{+}$ (calculated $=911.25$ ).

[4c] $\left(\mathrm{BF}_{4}\right)_{3} .{ }^{1} \mathrm{H}$ NMR $\left(\mathrm{CD}_{3} \mathrm{CN}, 400 \mathrm{MHz}\right): \delta 4.36(\mathrm{~s}, 9 \mathrm{H}), 4.41(\mathrm{~s}, 6 \mathrm{H}), 8.21-8.32(\mathrm{~m}, 9 \mathrm{H}), 8.87(\mathrm{~s}$, 3H). ${ }^{13} \mathrm{C} \mathrm{NMR}\left(\mathrm{CD}_{3} \mathrm{CN}, 100 \mathrm{MHz}\right): \delta 39.9\left(\mathrm{~s}, \mathrm{~N}\left(\mathrm{CH}_{3}\right)\right), 47.7\left(\mathrm{~s}, \mathrm{CH}_{2}\right), 122.6$ (q, J = $\left.273 \mathrm{~Hz}, \mathrm{CF}_{3}\right), 123.3$ $\left(q, J=271 \mathrm{~Hz}, \mathrm{CF}_{3}\right), 127.8\left(\mathrm{q}, J=4 \mathrm{~Hz}\right.$, aryl CH), $130.2\left(\mathrm{q}, J=34 \mathrm{~Hz}, \mathrm{C}\left(\mathrm{CF}_{3}\right)\right), 130.6(\mathrm{q}, J=4 \mathrm{~Hz}$, aryl $\mathrm{CH}), 131.5$ (q, J = $4 \mathrm{~Hz}$, aryl CH), 133.0 (s, Trz CH), 133.3 (s, C), 136.1 (q, J = 35 Hz, C(CF $)$ ), 142.5(s, C). ${ }^{19} \mathrm{~F} \mathrm{NMR}\left(\mathrm{CD}_{3} \mathrm{CN}, 376 \mathrm{MHz}\right): \delta-59.8,-64.0,-151.6$. ESI-MS (313 K): $\mathrm{m} / z 1115.2\left\{[4 \mathrm{c}]\left(\mathrm{BF}_{4}\right)_{2}\right\}^{+}$ (calculated $=1115.2$ ).

[4d] $\left(\mathrm{BF}_{4}\right)_{3} .{ }^{1} \mathrm{H} N M R\left(\mathrm{CD}_{3} \mathrm{CN}, 400 \mathrm{MHz}\right): \delta 4.35(\mathrm{~s}, 9 \mathrm{H}), 4.42(\mathrm{~s}, 6 \mathrm{H}), 8.37(\mathrm{~s}, 3 \mathrm{H}), 8.55(\mathrm{~s}, 6 \mathrm{H}), 9.12$ (s, 3H). ${ }^{13} \mathrm{C} \mathrm{NMR}\left(\mathrm{CD}_{3} \mathrm{CN}, 100 \mathrm{MHz}\right): \delta 39.7$ (s, N(CH$)$ ), $47.7\left(\mathrm{~s}, \mathrm{CH}_{2}\right), 123.5$ (q, J = $\left.270 \mathrm{~Hz}, \mathrm{CF}_{3}\right)$, 124.3 (q, $J=4 \mathrm{~Hz}, \mathrm{o}-\mathrm{CH}), 126.6-127.0(\mathrm{~m}, \mathrm{p}-\mathrm{CH}), 129.5$ (s, $\operatorname{Trz} \mathrm{CH}), 134.1$ (q, J=35 Hz, C(CF $)$ ), 
$137.1(\mathrm{~s}, \mathrm{C}), 143.0(\mathrm{~s}, \mathrm{C}) .{ }^{19} \mathrm{~F} \mathrm{NMR}\left(\mathrm{CD}_{3} \mathrm{CN}, 376 \mathrm{MHz}\right): \delta$-63.6, -151.5. ESI-MS (313 K): m/z 1115.2 $\left\{[\mathbf{4 d}]\left(\mathrm{BF}_{4}\right)_{2}\right\}^{+}$(calculated $\left.=1115.2\right)$.

[5a] $\left(\mathrm{BF}_{4}\right)_{4}$. Inside an Ar filled glovebox, a Schlenk tube was charged with 1 equiv of $\mathbf{3 a}(0.500 \mathrm{~g})$, 6 equiv of $\left[\mathrm{Me}_{3} \mathrm{O}\right] \mathrm{BF}_{4}$, and a magnetic stirring bar. Dry dichloromethane $(12 \mathrm{~mL})$ was added to the flask and the resulting mixture stirred overnight, at ambient temperatures. Subsequently, the reaction was quenched with methanol $(13 \mathrm{~mL})$, after which it was allowed to stir for $1 \mathrm{hr}$. Next, all volatiles were removed using a rotary evaporator and the residue was triturated with dichloromethane $(10 \mathrm{~mL})$. Filtration gave a solid that was washed sequentially with dichloromethane and diethyl ether, and dried in vacuo. This gave the product $[5 a]\left(\mathrm{BF}_{4}\right)_{4}$ as a white solid (0.280 g, 34 \%). ${ }^{1} \mathrm{H}$ NMR (DMSO-d $6,400 \mathrm{MHz}$ ): $\delta 2.13$ (s, 18H), $2.38(\mathrm{~s}, 9 \mathrm{H}), 3.15$ (s, 3H), 4.68 (s, 9H), 5.77 (s, 6H), $7.26(\mathrm{~s}, 6 \mathrm{H}), 9.64(\mathrm{~s}, 3 \mathrm{H}) .{ }^{13} \mathrm{C}$ NMR (DMSO-d, $100 \mathrm{MHz}$ ): $\delta 16.9$ (o$\left.\mathrm{CH}_{3}\right), 20.7\left(\mathrm{p}-\mathrm{CH}_{3}\right), 39.7\left(\mathrm{~N}^{\mathrm{Amine}}\left(\mathrm{CH}_{3}\right)\right), 53.9\left(\mathrm{CH}_{2}\right), 129.8(\mathrm{p}-\mathrm{CH}), 130.8(\operatorname{Trz} \mathrm{CH}), 131.9\left(\mathrm{C}\left(\mathrm{m}-\mathrm{CH}_{3}\right)\right)$, $134.4\left(\mathrm{C}\left(\mathrm{o}-\mathrm{CH}_{3}\right)\right), 136.8$ (C), 142.4 (C). ESI-MS (313 K): $\mathrm{m} / \mathrm{z} 935.40\left\{[5 \mathrm{a}]\left(\mathrm{BF}_{4}\right)_{3}\right\}^{+}$(calculated = 935.46).

\section{Synthesis of $[4 a-d](O T f)_{3}$}

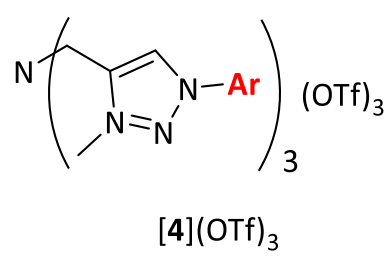

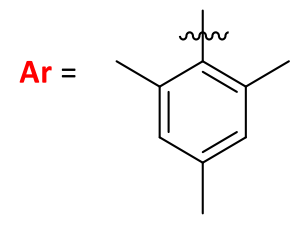

$4 a$

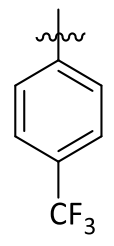

$4 b$

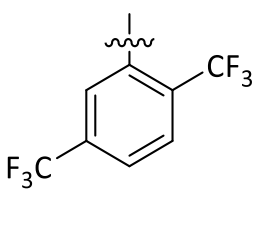

4c<smiles>CC(C)(C)c1cc(C(F)(F)F)cc(C(F)(F)F)c1</smiles>

4d

Solution State. To an argon-filled flame-dried round-bottom flask containing 1 equiv of 3 (0.500 g) dissolved in dry dichloromethane $(8-12 \mathrm{~mL}), 4.5$ equiv of MeOTf was injected at a slow, consistent pace. The resulting mixture was left to stir overnight. Subsequently, all volatiles were removed using a rotary evaporator and the residue was dried in vacuo at $60^{\circ} \mathrm{C}$, for 4 hours. Pure $[4 \mathrm{a}](\mathrm{OTf})_{3}$ was obtained by trituration with $\mathrm{EtOH}$, removing the supernatant by filtration, and washing the resulting solid with diethyl ether. This solid was dried in vacuo to give a white powder. Crude $[\mathbf{4 b}](\mathrm{OTf})_{3}$ and $[\mathbf{4 d}](\mathrm{OTf})_{3}$ were purified in analogous fashion to $[\mathbf{4 a}](\mathrm{OTf})_{3}$, except that trituration was performed using a minimal amount of cold THF, rather than EtOH. [4a] $(\mathrm{OTf})_{3}$, [4b](OTf $)_{3}$ and [4d](OTf) $)_{3}$ were isolated as white solids in best yields of $33 \%(0.297 \mathrm{~g}), 30 \%(0.257$ g) and $33 \%(0.257 \mathrm{~g})$, respectively. 
Solid State. A $10 \mathrm{~mL}$ stainless steel milling jar was charged $1 \times 10 \mathrm{~mm}$ stainless steel balls, $0.5 \mathrm{~g}$ (1 equiv) of 3, and 4.5 equiv of methyl triflate. The mixture is subjected to $25 \mathrm{~Hz}$ milling for $3 \mathrm{~h}$. Subsequently, the reaction mixture was dissolved in methanol $(10-13 \mathrm{~mL})$ and transferred to a round bottom flask, after which all volatiles were removed using a rotary evaporator. [4a](OTf) 3 was obtained by triturating the resulting residue with EtOH and filtering off the solution and washing with diethyl ether. The solid obtained was further dried until vacuo to give a white solid. $[\mathbf{4 b}](\mathrm{OTf})_{3}$ and $[\mathbf{4 d}](\mathrm{OTf})_{3}$ were obtained by triturating the residue with a minimal amount of cold THF and the solids obtained were isolated by filtration, washed with diethyl ether, and dried in vacuo. [4a](OTf) $)_{3},[\mathbf{4 b}](\mathrm{OTf})_{3}$ and [4d](OTf) $)_{3}$ were isolated as white solid in yields of $62 \%(0.558$ g), $24 \%(0.206 \mathrm{~g})$ and $25 \%(0.195 \mathrm{~g})$, respectively. These yields were not optimized and in the latter two compounds should be viewed as being, essentially, the same as those obtained from the corresponding solution-state reactions. All attempts to purify the crude reaction mixture for $3 c$ failed and, as a consequence, we were unable to cleanly isolate [4c](OTf) 3 .

[4a](OTf) ${ }_{3}{ }^{1} \mathrm{H}$ NMR (CD $\left.3 \mathrm{CN}, 400 \mathrm{MHz}\right): \delta 2.06(\mathrm{~s}, 18 \mathrm{H}), 2.38(\mathrm{~s}, 9 \mathrm{H}), 4.28(\mathrm{~s}, 9 \mathrm{H}), 4.41(\mathrm{~s}, 6 \mathrm{H}), 7.16$ (s, 6H), $8.72(\mathrm{~s}, 3 \mathrm{H}) .{ }^{19} \mathrm{~F} \mathrm{NMR}\left(\mathrm{CD}_{3} \mathrm{CN}, 376 \mathrm{MHz}\right): \delta-79.3$.

[4b](OTf) 3. ${ }^{1} \mathrm{H}$ NMR $\left(\mathrm{CD}_{3} \mathrm{CN}, 400 \mathrm{MHz}\right): \delta 4.34(\mathrm{~s}, 9 \mathrm{H}), 4.42(\mathrm{~s}, 6 \mathrm{H}), 8.03(\mathrm{~d}, J=8 \mathrm{~Hz}, 6 \mathrm{H}), 8.17(\mathrm{~d}, J$ $=8 \mathrm{~Hz}, 6 \mathrm{H}), 9.22(\mathrm{~s}, 3 \mathrm{H}) .{ }^{19} \mathrm{~F} \mathrm{NMR}\left(\mathrm{CD}_{3} \mathrm{CN}, 376 \mathrm{MHz}\right): \delta-63.4,-79.3$.

[4d](OTf) ${ }^{1}{ }^{1} \mathrm{H}$ NMR (CD $\left.3 \mathrm{CN}, 400 \mathrm{MHz}\right): \delta 4.36(\mathrm{~s}, 9 \mathrm{H}), 4.44(\mathrm{~s}, 6 \mathrm{H}), 8.37(\mathrm{~s}, 3 \mathrm{H}), 8.57(\mathrm{~s}, 6 \mathrm{H}), 9.27$ (s, 3H). ${ }^{19} \mathrm{~F}$ NMR $\left(\mathrm{CD}_{3} \mathrm{CN}, 376 \mathrm{MHz}\right): \delta-63.5,-79.4$.

[5d](OTf $)_{4}$. The side-product [5d](OTf $)_{4}$ was obtained as a clean compound from the solution state reaction of $\mathbf{3} \mathbf{d}$, by excluding the THF washing step in the above procedure and, instead, recrystallizing the crude solid by layering acetonitrile solutions of it with diethyl ether. This afforded [5d](OTf) $)_{4}$ as white crystals. ${ }^{1} \mathrm{H}$ NMR $\left(\mathrm{CD}_{3} \mathrm{CN}, 400 \mathrm{MHz}\right): \delta 3.71(\mathrm{~s}, 3 \mathrm{H}), 4.75(\mathrm{~s}, 9 \mathrm{H}), 5.52$ $(\mathrm{s}, 6 \mathrm{H}), 8.40(\mathrm{~s}, 3 \mathrm{H}), 8.60(\mathrm{~s}, 6 \mathrm{H}), 9.67(\mathrm{~s}, 3 \mathrm{H}) .{ }^{13} \mathrm{C} \mathrm{NMR}\left(\mathrm{CD}_{3} \mathrm{CN}, 100 \mathrm{MHz}\right): \delta 41.3\left(\mathrm{~s}, \mathrm{~N}\left(\mathrm{CH}_{3}\right)\right), 47.0$ (s, N $\left.{ }^{\text {Amine }}\left(\mathrm{CH}_{3}\right)\right), 55.4\left(\mathrm{~s}, \mathrm{CH}_{2}\right), 121.5\left(\mathrm{q}, J=317 \mathrm{~Hz}, \mathrm{CF}_{3}\right), 123.5\left(\mathrm{q}, J=271 \mathrm{~Hz}, \mathrm{CF}_{3}\right), 124.6(\mathrm{q}, J=3$ Hz, o-CH), 127.3 - 127.5 (m, p-CH), 132.5 (s, C), 134.3 (q, J = 34 Hz, C(CF 3$)), 135.4$ (s, Trz CH), 136.8 (s, C). ${ }^{19} \mathrm{~F}$ NMR $\left(\mathrm{CD}_{3} \mathrm{CN}, 376 \mathrm{MHz}\right): \delta$-63.6, -79.3. ESI-MS (313 K): $\mathrm{m} / \mathrm{z} 1402.9\left\{[5 \mathrm{~d}](\mathrm{OTf})_{3}\right\}^{+}$ (calculated $=1403.1$ ). 
[6c](OTf). Solution-state reaction of 3c with MeOTf gave the intended product, $[4 c](\mathrm{OTf})_{3}$, as a minor species. Despite repeated attempts, we were unable to cleanly isolate it. However, the major product [6c](OTf) (Figure S1, above) was obtained as a white solid by washing the crude material with MeOH. ${ }^{1} \mathrm{H}$ NMR $\left(\mathrm{CD}_{3} \mathrm{CN}, 400 \mathrm{MHz}\right): \delta 3.15(\mathrm{~s}, 3 \mathrm{H}), 4.79(\mathrm{~s}, 6 \mathrm{H}), 8.12(\mathrm{~s}, 3 \mathrm{H}), 8.15-$ $8.25(\mathrm{~m}, 6 \mathrm{H}), 8.78(\mathrm{~s}, 3 \mathrm{H})$. ESI-MS $(313 \mathrm{~K}): \mathrm{m} / z 911.06\{[6 \mathrm{c}](\mathrm{OTf})\}^{+}$(calculated = 911.17).

\subsection{General synthetic procedure for $\left[\mathrm{Ag}_{3}(1)_{2}\right]\left[B F_{4}\right]_{3}$}

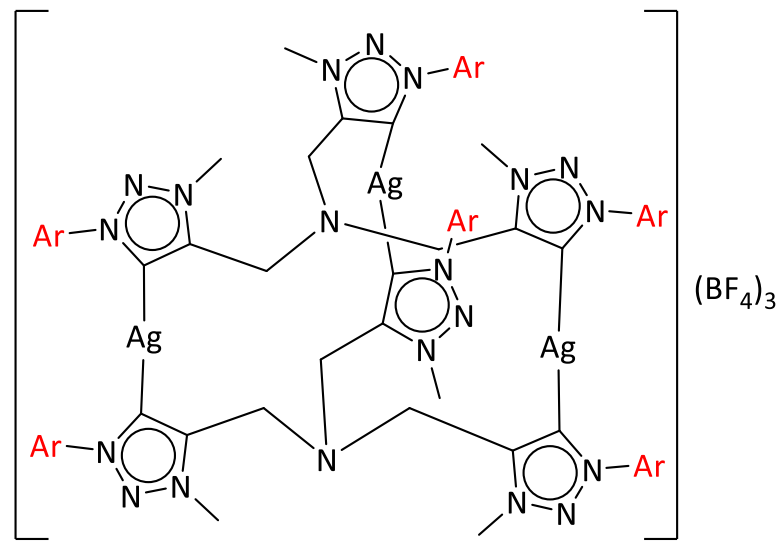

$\left[\mathrm{Ag}_{3}(1)_{2}\right]\left[\mathrm{BF}_{4}\right]_{3}$

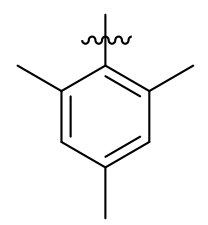

$\operatorname{Ar}=$

$1 a$<smiles>Cc1cc(C(F)(F)F)ccc1C(F)(F)F</smiles>

$1 c$<smiles>Cc1ccc(C(F)(F)F)cc1</smiles>

1b<smiles>CC(C)(C)c1cc(C(F)(F)F)cc(C(F)(F)F)c1</smiles>

$1 d$

Under an argon atmosphere, a flask was charged with $0.100 \mathrm{~g}$ (1 equiv) of [4](BF $)_{3}$, a 2-fold excess of silver(I) oxide ( 3 equiv) and $1 \mathrm{~mL}$ of Dimethyl sulfoxide, wrapped in aluminium foil, and heated overnight at $70^{\circ} \mathrm{C}$. Subsequent to cooling, the reaction mixture was filtered using a syringe filter (PTFE, $0.45 \mu \mathrm{m}$ ), and the filtrate was added to water. This caused precipitation of the product as a pale tan solid, which was isolated by filtration and rigorously dried under vacuum. Note, to avoid photodecomposition of the products, reaction and work-up were conducted in a darkened lab.

$\left[\mathrm{Ag}_{3}\left(\mathbf{1 a}_{\mathbf{2}}\right]\left[\mathrm{BF}_{4}\right]_{3}\right.$ was obtained as a pale tan solid $(0.093 \mathrm{~g}, 90 \%) .{ }^{1} \mathrm{H}$ NMR $\left(\mathrm{CD}_{3} \mathrm{CN}, 400 \mathrm{MHz}\right): \delta$ $1.81(\mathrm{~s}, 36 \mathrm{H}), 2.47(\mathrm{~s}, 18 \mathrm{H}), 3.92(\mathrm{~s}, 12 \mathrm{H}), 4.28(\mathrm{~s}, 18 \mathrm{H}), 6.95(\mathrm{~s}, 12 \mathrm{H}) .{ }^{13} \mathrm{C} \mathrm{NMR}\left(\mathrm{CD}_{3} \mathrm{CN}, 100 \mathrm{MHz}\right)$ : $\delta 17.3\left(\mathrm{o}-\mathrm{CH}_{3}\right), 21.4\left(\mathrm{p}-\mathrm{CH}_{3}\right), 38.3\left(\mathrm{~N}\left(\mathrm{CH}_{3}\right)\right), 52.7\left(\mathrm{CH}_{2}\right), 129.9(\mathrm{~m}-\mathrm{CH}), 134.8\left(\mathrm{C}\left(\mathrm{o}-\mathrm{CH}_{3}\right)\right), 136.6(\mathrm{C}(\mathrm{p}-$ $\left.\left.\mathrm{CH}_{3}\right)\right), 141.3(\mathrm{C}), 144.2(\mathrm{C}), 172.9$ (d, $\left.J=177 \mathrm{~Hz}, \mathrm{Ag}(\mathrm{C})\right) .{ }^{19} \mathrm{~F} \mathrm{NMR}\left(\mathrm{CD}_{3} \mathrm{CN}, 376 \mathrm{MHz}\right): \delta$-151.6. ESIMS (313 K): $m / z$ 1811.2 $\left\{\mathrm{Ag}_{3}[\mathbf{1 a}]_{2}\left(\mathrm{BF}_{4}\right)_{2}\right\}^{+}$(calculated $\left.=1811.5\right)$.

$\left[\mathrm{Ag}_{3}(\mathbf{1 b})_{2}\right]\left[\mathrm{BF}_{4}\right]_{3}$ was obtained as a pale tan solid $(0.188 \mathrm{~g}, 92 \%)$. Two species are observed in solution, which are in a solvent- and temperature-dependent equilibrium with one another; see 
Figures S55 - S59. The following data is for the major species present at room temperature. ${ }^{1} \mathrm{H}$ NMR (DMSO-d $6,400 \mathrm{MHz}$ ): $\delta 3.95$ (brs, 6H), 4.39 (s, 18H), 4.70 (brs, 6H), 7.95 (d, J = $8 \mathrm{~Hz}, 12 \mathrm{H}$ ), $8.26\left(\mathrm{~d}, J=4 \mathrm{~Hz}, 12 \mathrm{H}\right.$ ). ${ }^{13} \mathrm{C}$ NMR (DMSO-d, $100 \mathrm{MHz}$ ): $\delta 37.7\left(\mathrm{~s}, \mathrm{~N}\left(\mathrm{CH}_{3}\right)\right), 51.6\left(\mathrm{~s}, \mathrm{CH}_{2}\right), 123.5$ (q, J $\left.=273 \mathrm{~Hz}, \mathrm{CF}_{3}\right), 124.5(\mathrm{~s}, \operatorname{aryl} \mathrm{CH}), 127.0(\mathrm{~s}, \operatorname{aryl} \mathrm{CH}), 130.6\left(\mathrm{q}, J=33 \mathrm{~Hz}, \mathrm{C}\left(\mathrm{CF}_{3}\right)\right), 142.3(\mathrm{~s}, \mathrm{C}), 143.8$ (s, C), 167.8 (brs, $\mathrm{Ag}(\mathrm{C})) .{ }^{19} \mathrm{~F}$ NMR (DMSO-d, $\left.376 \mathrm{MHz}\right): \delta-61.7,-148.4$. ESI-MS (313 K): $\mathrm{m} / \mathrm{z}$ 1964.9 $\left\{\mathrm{Ag}_{3}[\mathbf{1} \mathbf{b}]_{2}\left(\mathrm{BF}_{4}\right)_{2}\right\}^{+}($calculated $=1965.1)$.

$\left[\mathrm{Ag}_{3}(\mathbf{1 c})_{2}\right]\left[\mathrm{BF}_{4}\right]_{3}$ was obtained as a pale tan solid $(0.186 \mathrm{~g}, 91 \%) .{ }^{1} \mathrm{H}$ NMR (DMSO-d $\left.6,400 \mathrm{MHz}\right): \delta$ $3.74(\mathrm{~d}, J=12 \mathrm{~Hz}, 6 \mathrm{H}), 4.33-4.40(\mathrm{~m}, 24 \mathrm{H}), 8.14-8.20(\mathrm{~m}, 12 \mathrm{H}), 8.30-8.35(\mathrm{~m}, 6 \mathrm{H}) .{ }^{13} \mathrm{C}$ NMR (DMSO-d $6,100 \mathrm{MHz}$ ): $\delta 37.7\left(\mathrm{~s}, \mathrm{~N}\left(\mathrm{CH}_{3}\right)\right.$ ), $51.5\left(\mathrm{~s}, \mathrm{CH}_{2}\right), 121.5$ (q, $\left.J=273 \mathrm{~Hz}, \mathrm{CF}_{3}\right), 122.3$ (q, J = 271 $\left.\mathrm{Hz}, \mathrm{CF}_{3}\right), 126.6$ - $126.9\left(\mathrm{~m}\right.$, aryl CH), 128.3 (q, $\left.33 \mathrm{~Hz}, \mathrm{C}\left(\mathrm{CF}_{3}\right)\right), 128.8-129.1$ (m, aryl CH), 129.2 $129.5(\mathrm{~m}, \operatorname{aryl~CH}), 133.9$ (q, J = $\left.34 \mathrm{~Hz}, \mathrm{C}\left(\mathrm{CF}_{3}\right)\right), 137.4$ (s, C), 143.2 (d, J = $11 \mathrm{~Hz}, \mathrm{Trz}$ C), 172.8 (d, J $=166 \mathrm{~Hz}, \mathrm{Ag}(\mathrm{C})) .{ }^{19} \mathrm{~F}$ NMR (DMSO-d $6,376 \mathrm{MHz}$ ): $\delta$ - 59.0, -62.1, -148.4. ESI-MS (313 K): $\mathrm{m} / \mathrm{z} 2374.8$ $\left\{\mathrm{Ag}_{3}[\mathbf{1 c}]_{2}\left(\mathrm{BF}_{4}\right)_{2}\right\}^{+}($calculated $=2375.1)$.

$\left[\mathrm{Ag}_{3}(\mathbf{1 d})_{2}\right]\left[\mathrm{BF}_{4}\right]_{3}$ was obtained as a pale tan solid $(0.188 \mathrm{~g}, 92 \%) .{ }^{1} \mathrm{H}$ NMR (DMSO-d 6 , $\left.400 \mathrm{MHz}\right): \delta$ $3.95(\mathrm{~d}, J=16 \mathrm{~Hz}, 6 \mathrm{H}), 4.36(\mathrm{~s}, 18 \mathrm{H}), 4.64(\mathrm{~d}, J=16 \mathrm{~Hz}, 6 \mathrm{H}), 8.30(\mathrm{~s}, 6 \mathrm{H}), 8.60(\mathrm{~s}, 12 \mathrm{H}) .{ }^{13} \mathrm{C} N M R$ (DMSO-d $6,100 \mathrm{MHz}$ ): $\delta 37.6\left(\mathrm{~s}, \mathrm{~N}\left(\mathrm{CH}_{3}\right)\right.$ ), 51.7 (s, $\left.\mathrm{CH}_{2}\right), 122.3$ (q, J = $\left.272 \mathrm{~Hz}, \mathrm{CF}_{3}\right), 123.9$ - $124.1(\mathrm{~m}$, $\operatorname{aryl~CH),~} 124.1$ - 124.4 (m, aryl CH), 126.4 (s), 131.7 (q, J = 34 Hz, C(CF3)), 140.7 (s, C), 144.0 (s, C). ${ }^{19} \mathrm{~F} \mathrm{NMR}$ (DMSO-d $\left.6,376 \mathrm{MHz}\right): \delta-62.0,-148.4$. ESI-MS $(313 \mathrm{~K}): \mathrm{m} / z 2374.8\left\{\mathrm{Ag}_{3}[1 \mathrm{~d}]_{2}\left(\mathrm{BF}_{4}\right)_{2}\right\}^{+}$ (calculated $=2375.1$ ).

\subsection{General synthetic procedure for $\left[\mathrm{Ag}_{3}(1)_{2}\right][\mathrm{OTf}]_{3}$}

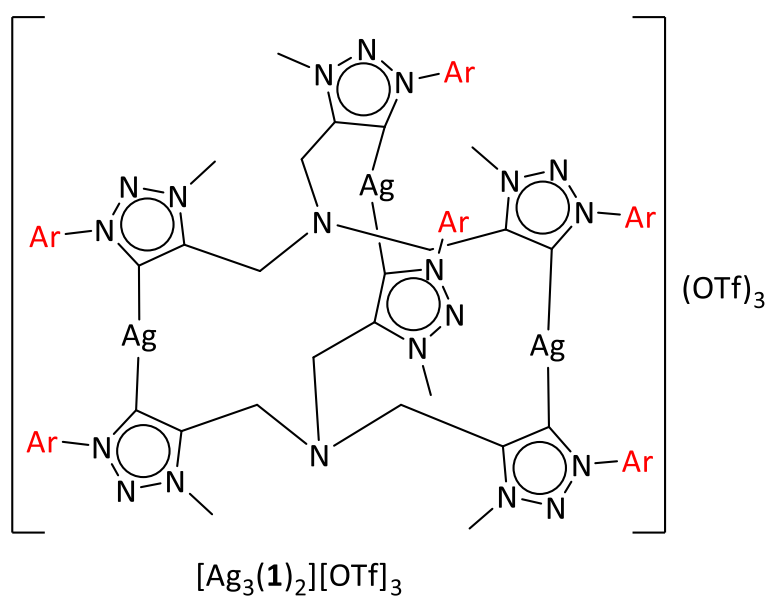

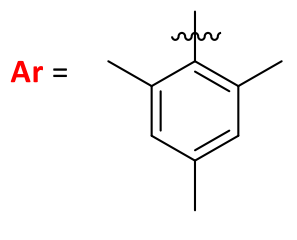

$1 a$

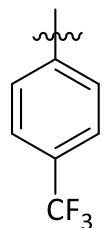

1b

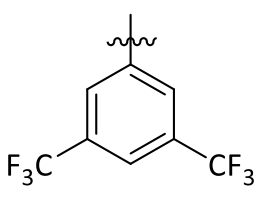

1d 
Under an argon atmosphere, $5 \mathrm{~mL}$ of acetonitrile was added to a Schlenk flask containing 0.100 $\mathrm{g}$ (1 equiv) of [4](OTf) 3 and a 2-fold excess of silver(I) oxide (3 equiv). The flask was then wrapped in aluminium foil and heated at $70^{\circ} \mathrm{C}$ overnight. Upon cooling, the reaction mixture was filtered through celite and the celite washed with acetonitrile. All volatiles were removed from the combined filtrates in vacuo and the residue, thereby, obtained was extracted with dichloromethane. Subsequent to removal of unwanted solids by filtration, the filtrate was reduced to dryness in vacuo. Drying the resulting solid for 1 day in a desiccator containing $\mathrm{KOH}$ gave the desired products, $\left[\mathrm{Ag}_{3}(\mathbf{1} \mathbf{a})_{2}\right](\mathrm{OTf})_{3},\left[\mathrm{Ag}_{3}(\mathbf{1} \mathbf{b})_{2}\right](\mathrm{OTf})_{3}$ and $\left[\mathrm{Ag}_{3}(\mathbf{1 d})_{2}\right](\mathrm{OTf})_{3}$. Note, to avoid photodecomposition of the products, reaction and work-up were conducted in a darkened lab.

$\left[\operatorname{Ag}_{3}\left(\mathbf{1 a}_{2}\right)_{2}\right][0 T f]_{3}$ was obtained as a brownish-yellow solid $(0.089 \mathrm{~g}, 95 \%) .{ }^{1} \mathrm{H}$ NMR $\left(\mathrm{CD}_{3} \mathrm{CN}, 400\right.$ $\mathrm{MHz}): \delta 2.16(\mathrm{~s}, 18 \mathrm{H}), 2.46(\mathrm{~s}, 9 \mathrm{H}), 3.88(\mathrm{~s}, 6 \mathrm{H}), 4.24(\mathrm{~s}, 9 \mathrm{H}), 6.49(\mathrm{~s}, 6 \mathrm{H}) .{ }^{19} \mathrm{~F} \mathrm{NMR}\left(\mathrm{CD}_{3} \mathrm{CN}, 376\right.$ $\mathrm{MHz}): \delta-79.24$.

$\left[\mathrm{Ag}_{3}(\mathbf{1 b})_{2}\right][\mathrm{OTf}]_{3}$ was obtained as a brownish-yellow solid $(0.087 \mathrm{~g}, 92 \%) .{ }^{1} \mathrm{H} \mathrm{NMR}\left(\mathrm{CD}_{3} \mathrm{CN}, 400\right.$ $\mathrm{MHz}): \delta 3.92(\mathrm{~s}, 6 \mathrm{H}), 4.27(\mathrm{~s}, 9 \mathrm{H}), 7.40(\mathrm{~d}, 6 \mathrm{H}), 7.66(\mathrm{~d}, 6 \mathrm{H}), 7.83(\mathrm{~d}, 6 \mathrm{H}), 8.11(\mathrm{~d}, 6 \mathrm{H}) .{ }^{19} \mathrm{~F} \mathrm{NMR}$ $\left(\mathrm{CD}_{3} \mathrm{CN}, 376 \mathrm{MHz}\right): \delta-63.11,-63.29,-79.25$.

$\left[\mathrm{Ag}_{3}(\mathbf{1 d})_{2}\right][\mathrm{OTf}]_{3}$ was obtained as a brownish-yellow solid $(0.088 \mathrm{~g}, 93 \%) .{ }^{1} \mathrm{H}$ NMR $\left(\mathrm{CD}_{3} \mathrm{CN}, 400\right.$ $\mathrm{MHz}): \delta 4.29(\mathrm{~s}, 9 \mathrm{H}), 8.15(\mathrm{~s}, 3 \mathrm{H}), 8.41(\mathrm{~s}, 6 \mathrm{H}) .{ }^{19} \mathrm{~F} \mathrm{NMR}\left(\mathrm{CD}_{3} \mathrm{CN}, 376 \mathrm{MHz}\right): \delta-63.79,-79.28$. 


\subsection{Synthesis of cobalt complexes}

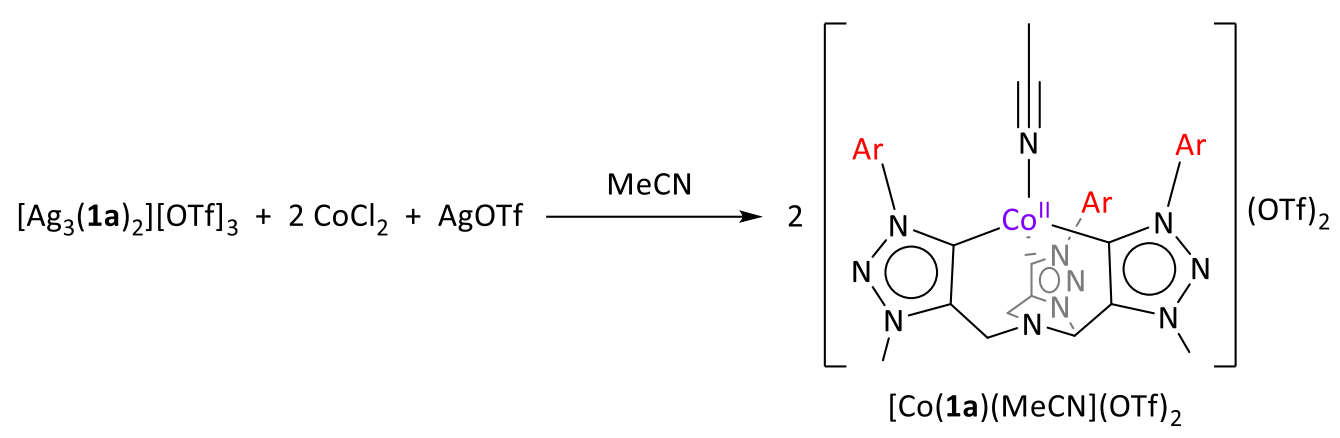

[Co(1a)(MeCN)](OTf) 2. Under an argon atmosphere, 2 equiv $\mathrm{CoCl}_{2}(0.0249 \mathrm{~g}, 0.192 \mathrm{mmol})$ and 1 equiv AgOTf $(0.0247 \mathrm{~g}, 0.0959 \mathrm{mmol})$ were added to a solution of $\left[\mathrm{Ag}_{3}(1 \mathrm{a})_{2}\right][\mathrm{OTf}]_{3}(0.200 \mathrm{~g}$, $0.0959 \mathrm{mmol})$ in acetonitrile $(5 \mathrm{~mL})$. The resulting mixture was stirred overnight. This gave a suspension, which was subsequently filtered, and the purple filtrate was reduced to dryness in vacuo to yield a purple solid $(0.173 \mathrm{~g}, 86 \%)$. Crystals suitable for X-ray crystallography were grown by slow diffusion of diethyl ether vapour into concentrated acetonitrile solutions of complex. ${ }^{1} \mathrm{H}$ NMR $\left(\mathrm{CD}_{3} \mathrm{CN}, 400 \mathrm{MHz}\right): \delta$-1.34 (9H), $3.30(9 \mathrm{H}), 5.06(18 \mathrm{H}), 6.99(6 \mathrm{H}), 79.32(6 \mathrm{H}) .{ }^{19} \mathrm{~F}$ NMR $\left(\mathrm{CD}_{3} \mathrm{CN}, 376 \mathrm{MHz}\right): \delta$-79.3. Evans' NMR (298 K): $\mu_{\text {eff }}=3.97$ B.M.

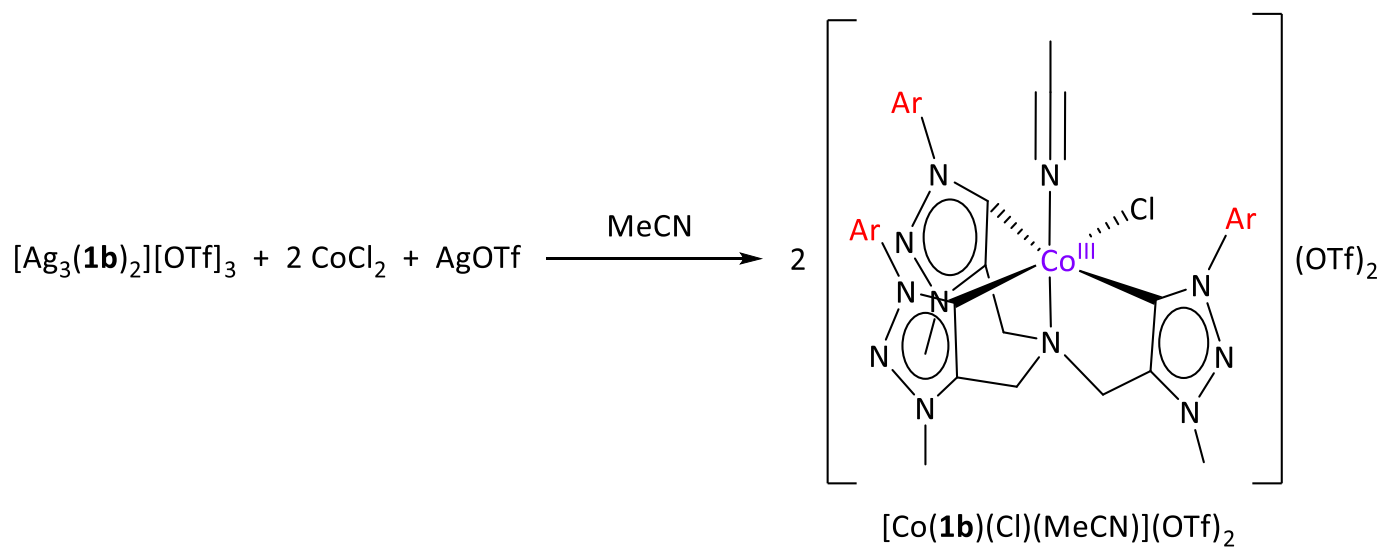

[Co(1b)(Cl)(MeCN)](OTf) 2. Under an argon atmosphere, 2 equiv $\mathrm{CoCl}_{2}(0.0232 \mathrm{~g}, 0.179 \mathrm{mmol})$ and 1 equiv AgOTf $(0.0229 \mathrm{~g}, 0.0893 \mathrm{mmol})$ were added to a solution of $\left[\mathrm{Ag}_{3}(\mathbf{1} \mathbf{b})_{2}\right][\mathrm{OTf}]_{3}(0.200$ $\mathrm{g}, 0.0959 \mathrm{mmol})$ in acetonitrile $(5 \mathrm{~mL})$. The resulting mixture was stirred overnight. This gave a suspension, which was subsequently filtered, and the filtrate was reduced to dryness in vacuo to provide a yellow solid. Attempts made to purify this material by washing and recrystallization using a wide range of solvents proved unsuccessful. However, red crystals suitable for X-ray crystallography were grown by slow diffusion of diethyl ether vapour into a concentrated solution of complex in acetonitrile. ${ }^{1} \mathrm{H} \mathrm{NMR}\left(\mathrm{CD}_{3} \mathrm{CN}, 400 \mathrm{MHz}\right): \delta 3.99(\mathrm{~s}, 3 \mathrm{H}), 4.10(\mathrm{~d}, J=12 \mathrm{~Hz}$, 
$2 \mathrm{H}), 4.26(\mathrm{~m}, 8 \mathrm{H}), 4.62(\mathrm{~d}, J=12 \mathrm{~Hz}, 2 \mathrm{H}), 6.93(\mathrm{~d}, J=8 \mathrm{~Hz}, 2 \mathrm{H}), 7.25(\mathrm{~d}, J=8 \mathrm{~Hz}, 2 \mathrm{H}), 7.84(\mathrm{~d}, J=8$ $\mathrm{Hz}, 4 \mathrm{H}), 7.93(\mathrm{~d}, J=8 \mathrm{~Hz}, 4 \mathrm{H}) .{ }^{19} \mathrm{~F} N M R\left(\mathrm{CD}_{3} \mathrm{CN}, 376 \mathrm{MHz}\right): \delta-63.5,-64.3,-79.3$.

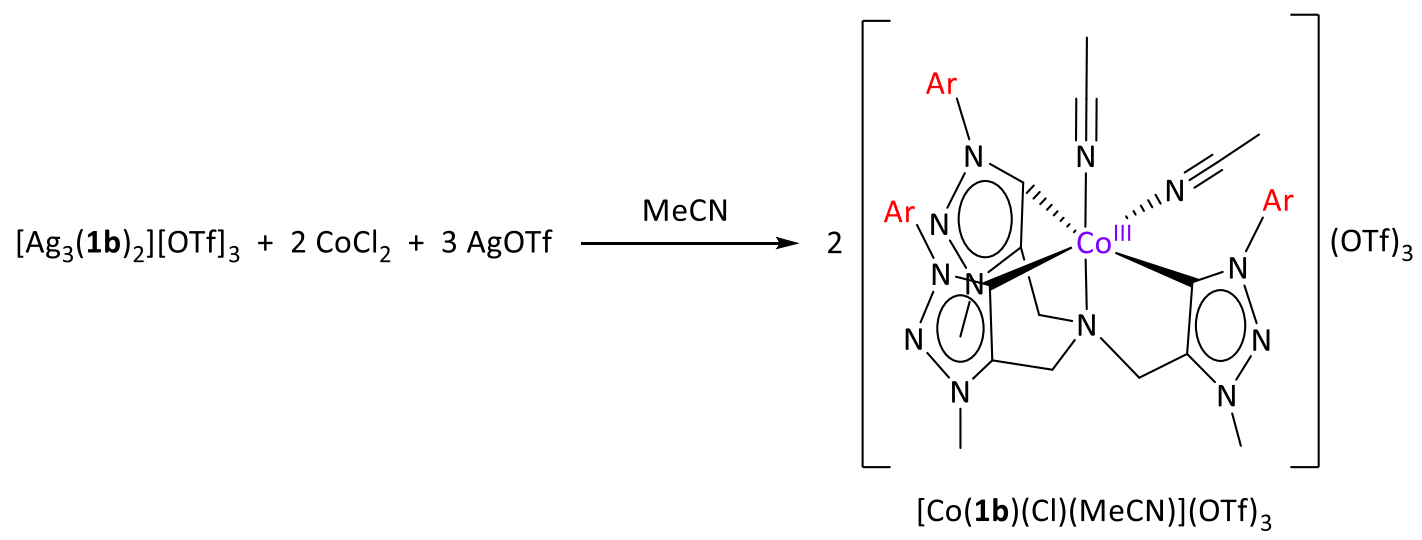

[Co(1b)(MeCN) $\left.)_{(\mathbf{O T f}}\right)_{3}$. Under an argon atmosphere, 2 equiv $\mathrm{CoCl}_{2}(0.0116 \mathrm{~g}, 0.893 \mathrm{mmol})$ and 1 equiv AgOTf $(0.0344 \mathrm{~g}, 0.134 \mathrm{mmol})$ were added to a solution of $\left[\mathrm{Ag}_{\mathbf{3}}(\mathbf{1} \mathbf{b})_{\mathbf{2}}\right][\mathbf{O T f}]_{3}(0.100 \mathrm{~g}$, $0.0446 \mathrm{mmol}$ ) in acetonitrile $(5 \mathrm{~mL})$. The resulting mixture was stirred overnight. This gave a suspension, which was subsequently filtered, and the filtrate was reduced to dryness in vacuo to provide an orange solid. Attempts made to purify this material by washing and recrystallization using a wide range of solvents proved unsuccessful. However, orange crystal suitable for X-ray crystallography were grown by layering diethyl ether onto a concentrated solution of complex in acetone. ${ }^{1} \mathrm{H}$ NMR (CD $\left.{ }_{3} \mathrm{CN}, 400 \mathrm{MHz}\right): \delta 1.16(\mathrm{~s}, 3 \mathrm{H}), 2.51(\mathrm{~s}, 3 \mathrm{H}), 4.00(\mathrm{~s}, 3 \mathrm{H}), 4.31(\mathrm{~s}, 6 \mathrm{H}), 4.38(\mathrm{~m}$, $4 \mathrm{H}), 4.58(\mathrm{~d}, J=12 \mathrm{~Hz}, 2 \mathrm{H}), 6.76(\mathrm{~d}, J=8 \mathrm{~Hz}, 2 \mathrm{H}), 7.22(\mathrm{~d}, J=8 \mathrm{~Hz}, 2 \mathrm{H}), 7.88(\mathrm{~d}, J=8 \mathrm{~Hz}, 4 \mathrm{H}), 7.97$ $(\mathrm{d}, J=8 \mathrm{~Hz}, 4 \mathrm{H}) .{ }^{19} \mathrm{~F}$ NMR $\left(\mathrm{CD}_{3} \mathrm{CN}, 376 \mathrm{MHz}\right): \delta-63.4,-64.3,-79.3$. 


\section{NMR Spectra}

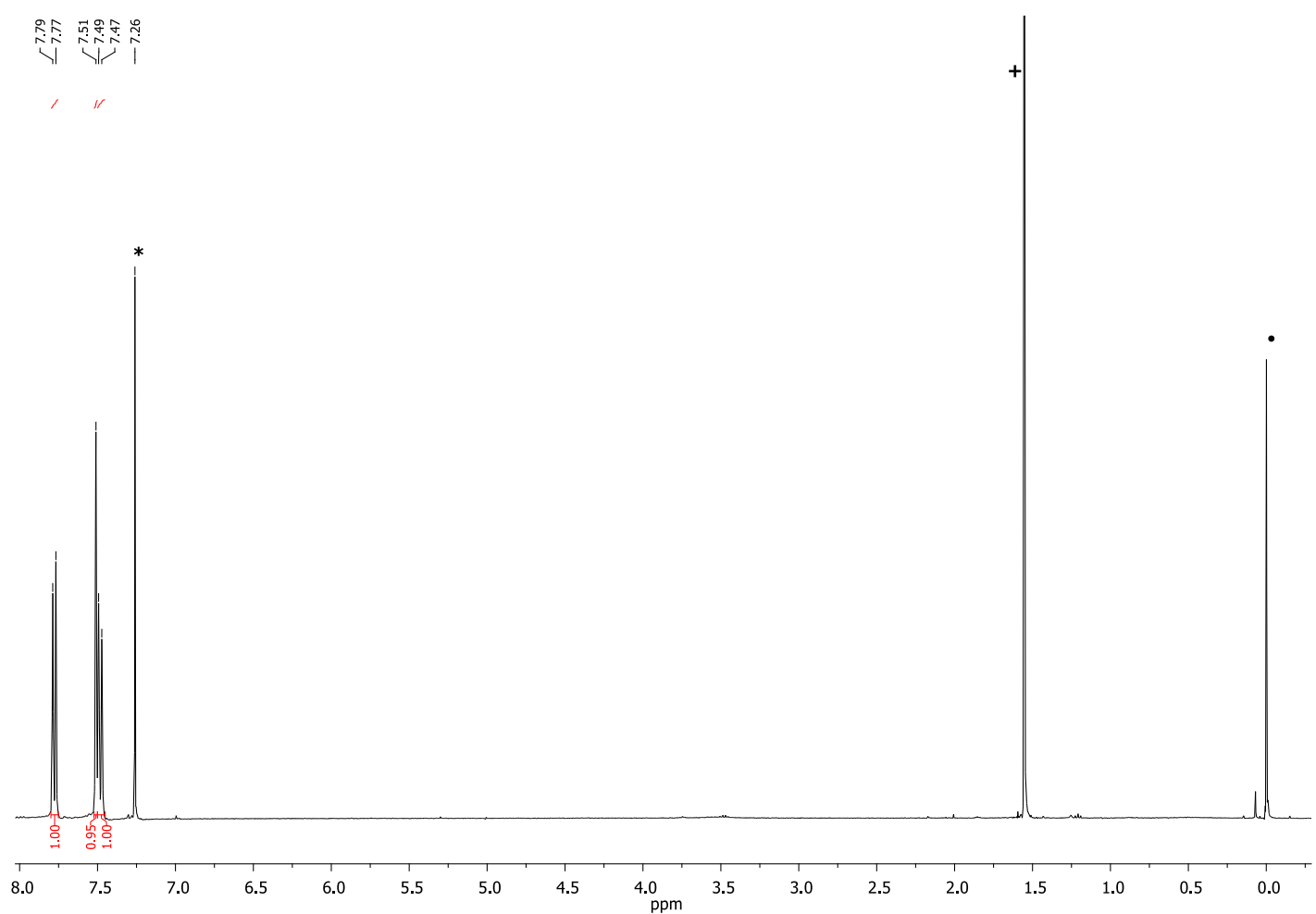

Figure S1. ${ }^{1} \mathrm{H}$ NMR spectrum of $2 \mathbf{c}$, recorded in $\mathrm{CDCl}_{3}$ solution $\left({ }^{*}=\mathrm{CDCl}_{3} ;+=\mathrm{H}_{2} \mathrm{O} ; \bullet=\mathrm{TMS}\right)$.

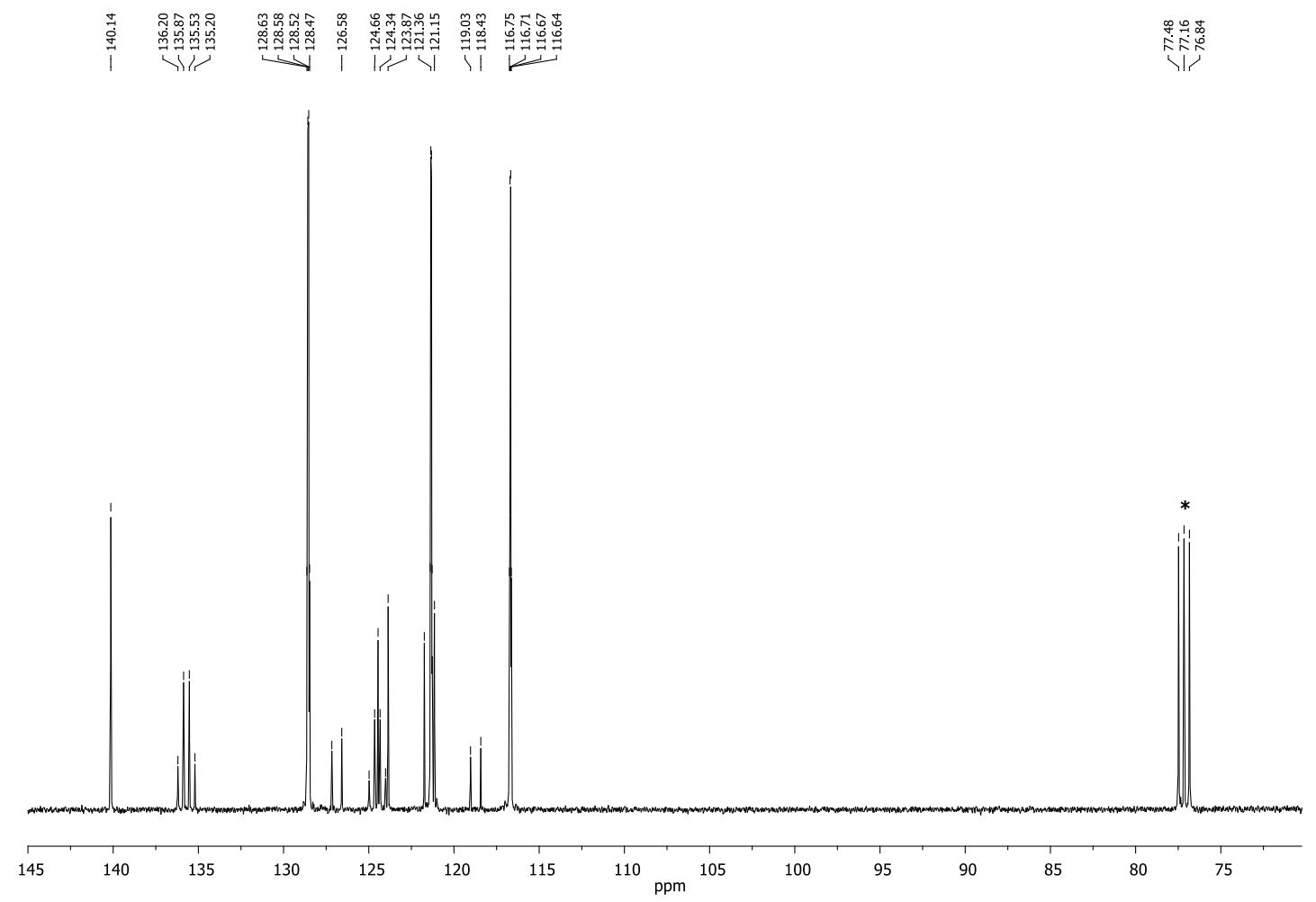

Figure $\mathbf{S 2} .{ }^{13} \mathrm{C}$ NMR spectrum of $\mathbf{2 c}$, recorded in $\mathrm{CDCl}_{3}\left({ }^{*}\right)$ solution. 


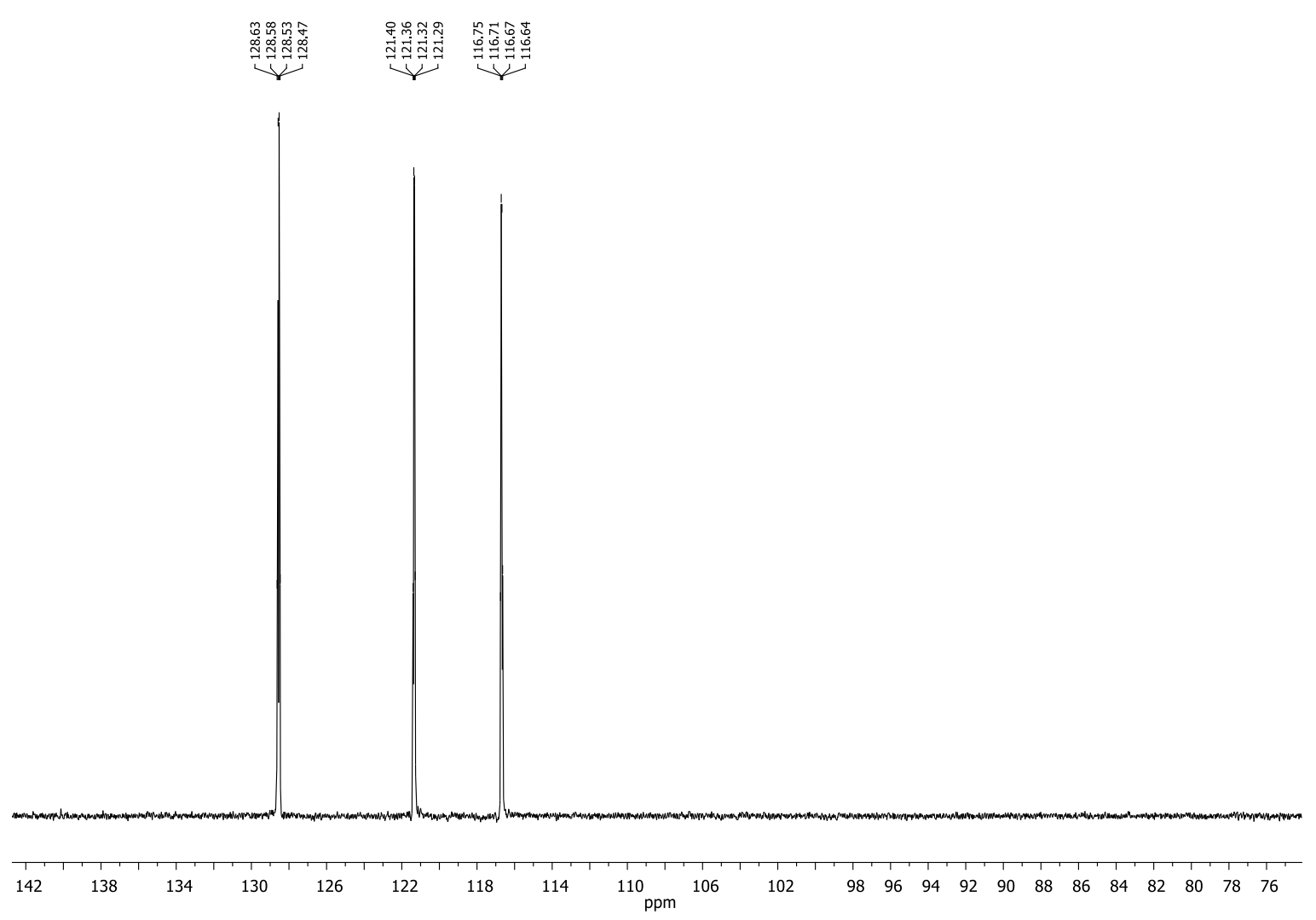

Figure S3. DEPT-135 NMR spectrum of $2 \mathbf{c}$, recorded in $\mathrm{CDCl}_{3}$ solution.

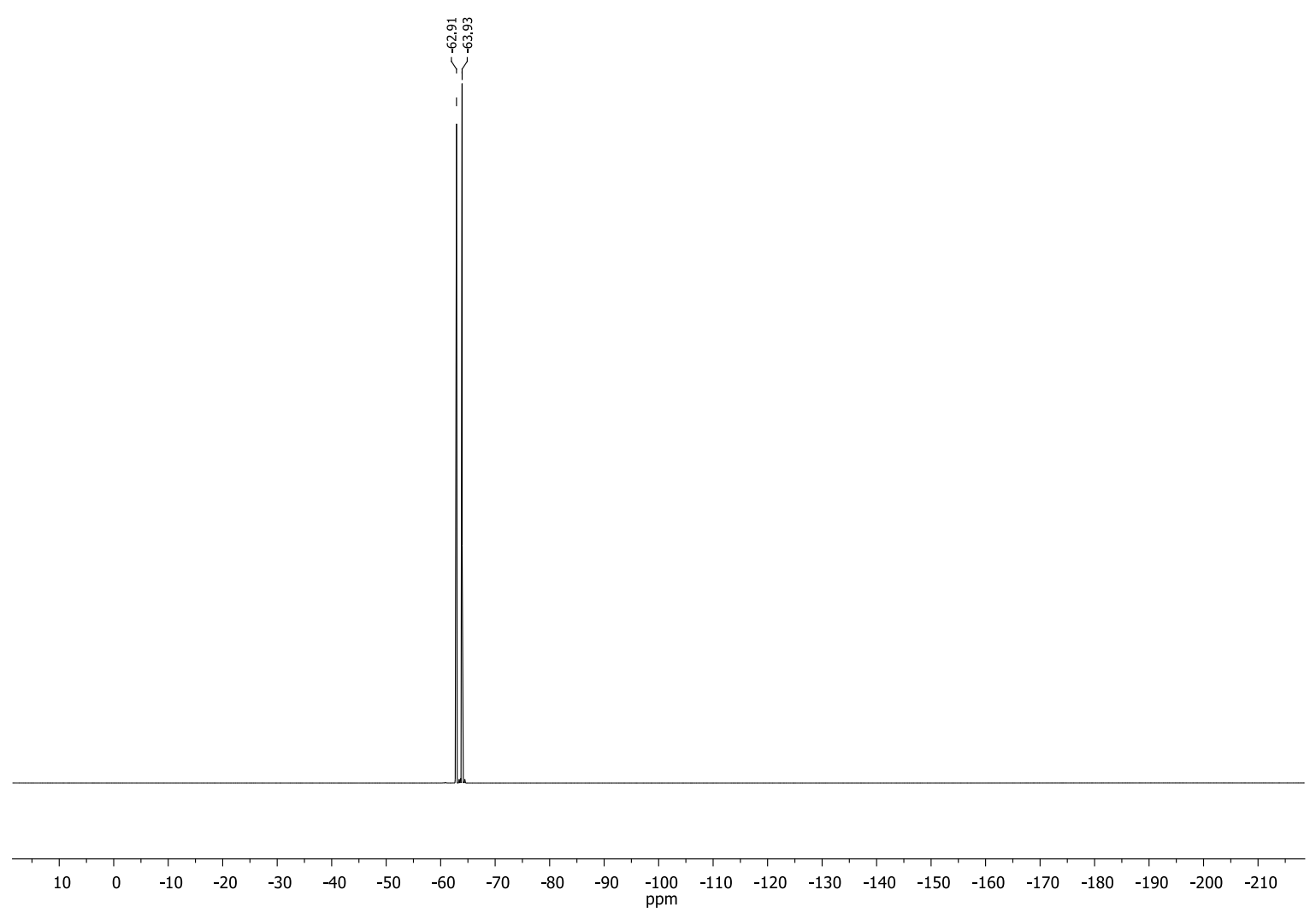

Figure $54 .{ }^{19} \mathrm{~F}$ NMR spectrum of $2 \mathrm{c}$, recorded in $\mathrm{CDCl}_{3}$ solution. 


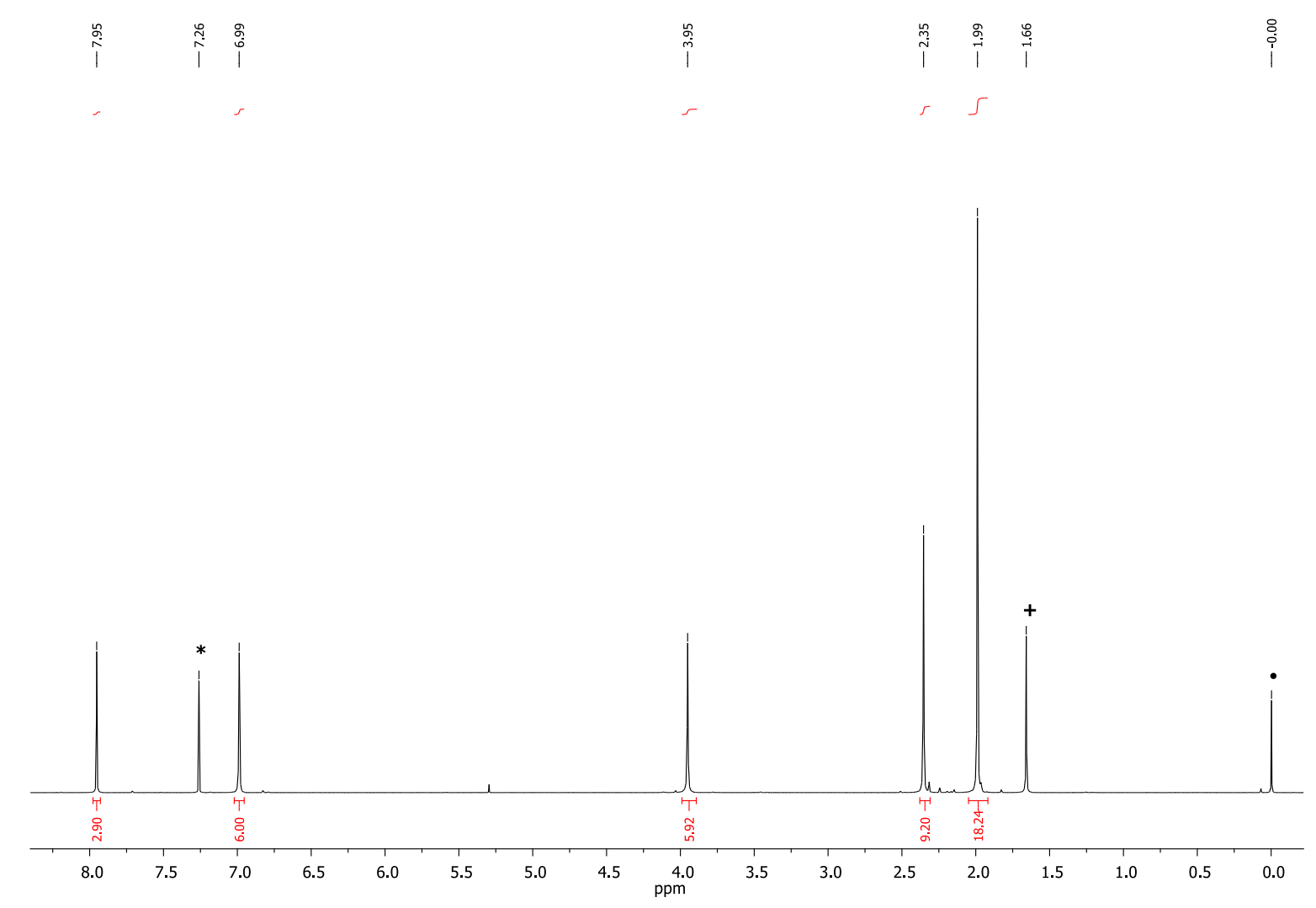

Figure S5. ${ }^{1} \mathrm{H}$ NMR spectrum of $3 a$, recorded in $\mathrm{CDCl}_{3}$ solution $\left({ }^{*}=\mathrm{CDCl}_{3} ;+=\mathrm{H}_{2} \mathrm{O} ; \bullet=\mathrm{TMS}\right)$.
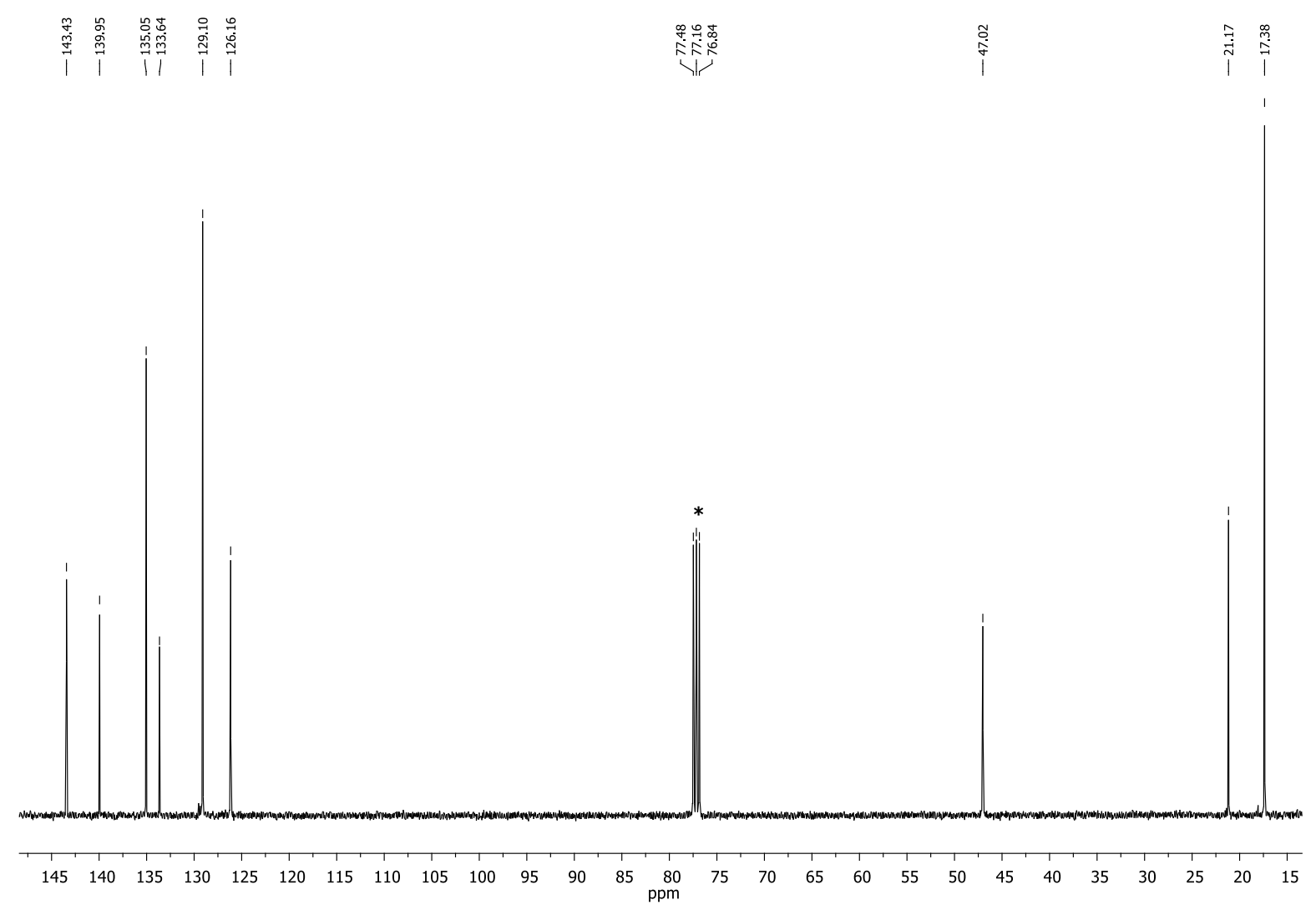

Figure S6. ${ }^{13} \mathrm{C}$ NMR spectrum of $3 a$, recorded in $\mathrm{CDCl}_{3}\left({ }^{*}\right)$ solution. 


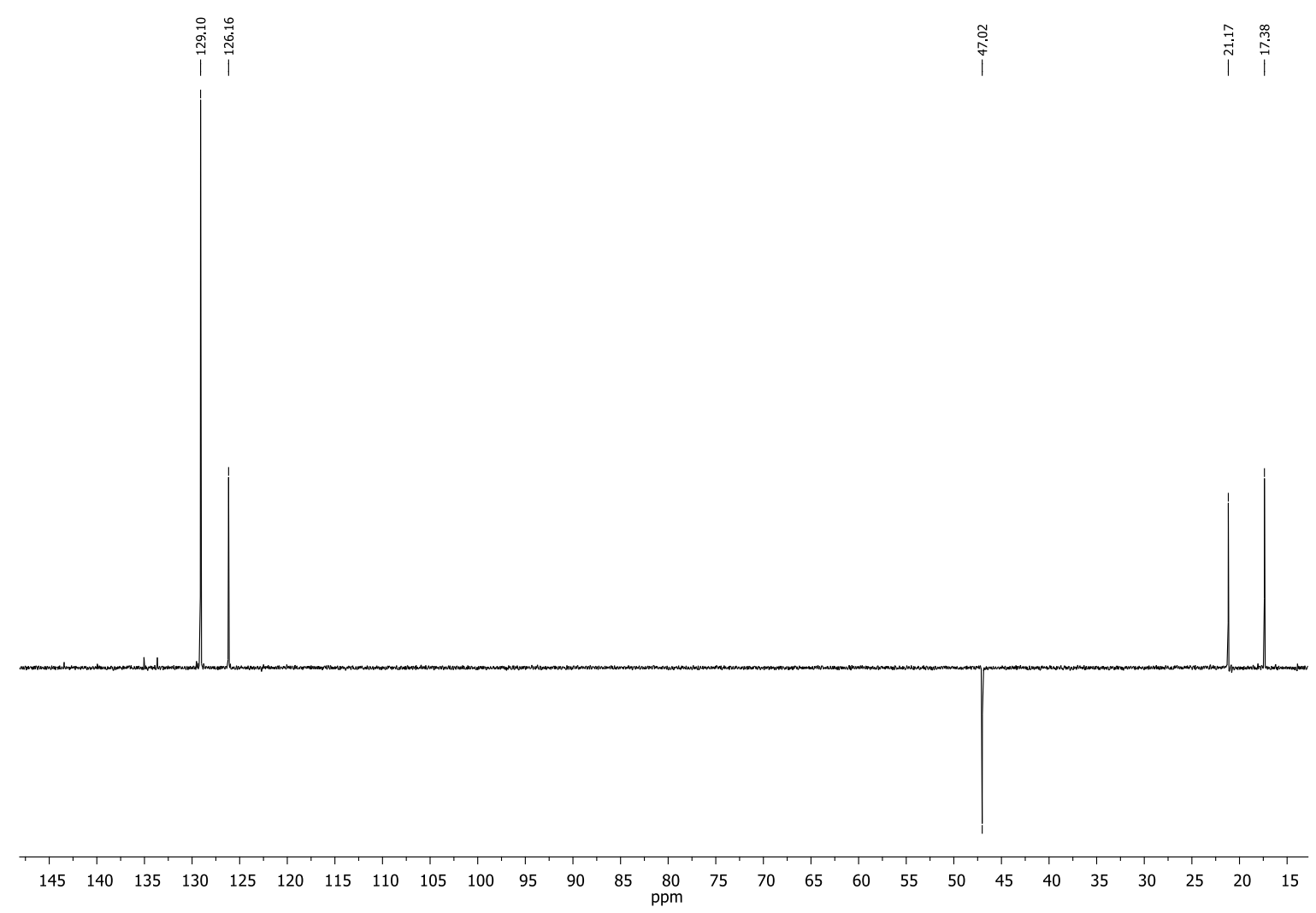

Figure S7. DEPT-135 NMR spectrum of 3 a, recorded in $\mathrm{CDCl}_{3}$ solution.

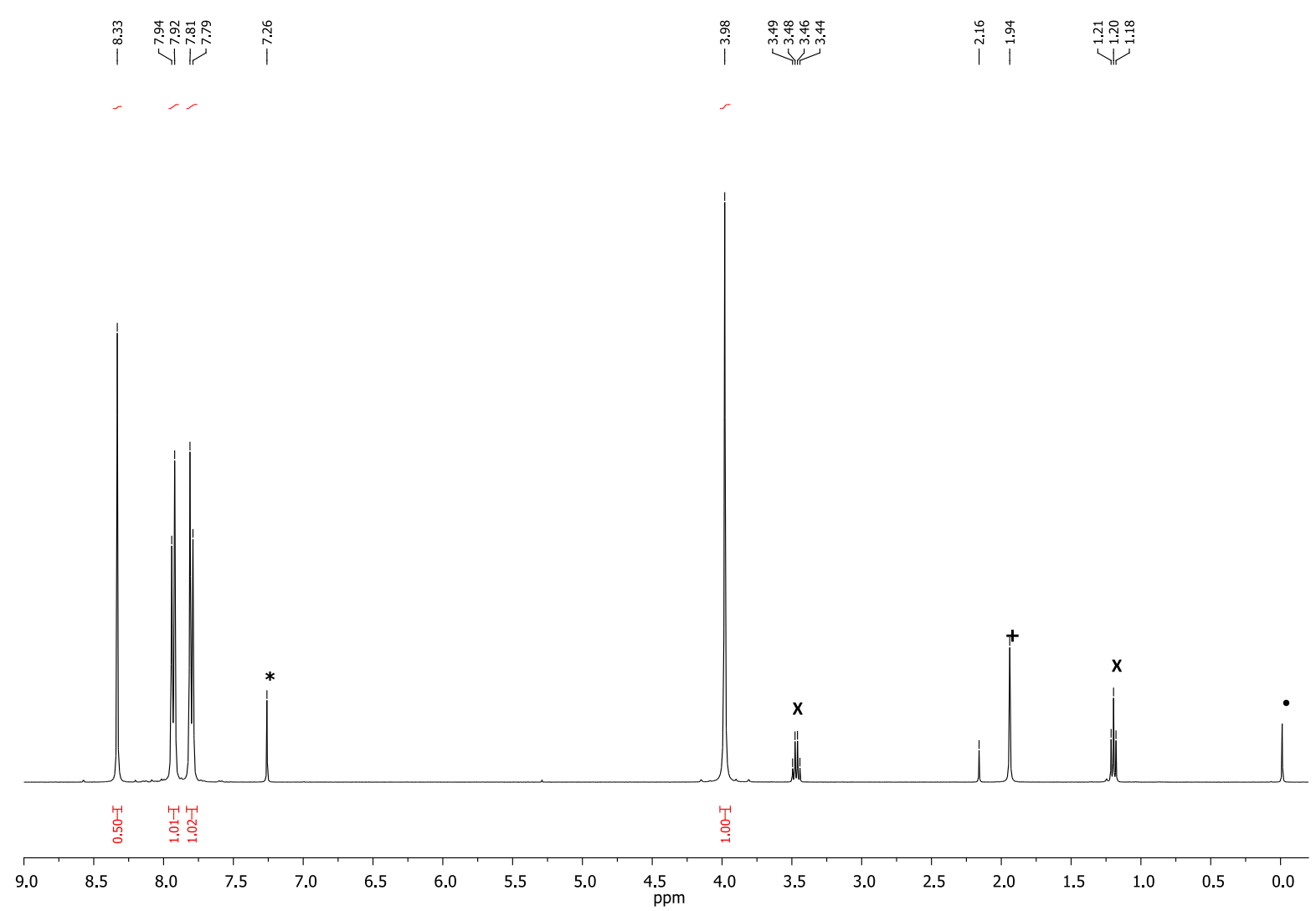

Figure S8. ${ }^{1} \mathrm{H}$ NMR spectrum of $\mathbf{3 b}$, recorded in $\mathrm{CDCl}_{3}$ solution $\left(*=\mathrm{CDCl}_{3} ;+=\mathrm{H}_{2} \mathrm{O} ; \bullet=\mathrm{TMS} \mathbf{x}=\mathrm{Et}_{2} \mathrm{O}\right)$. 


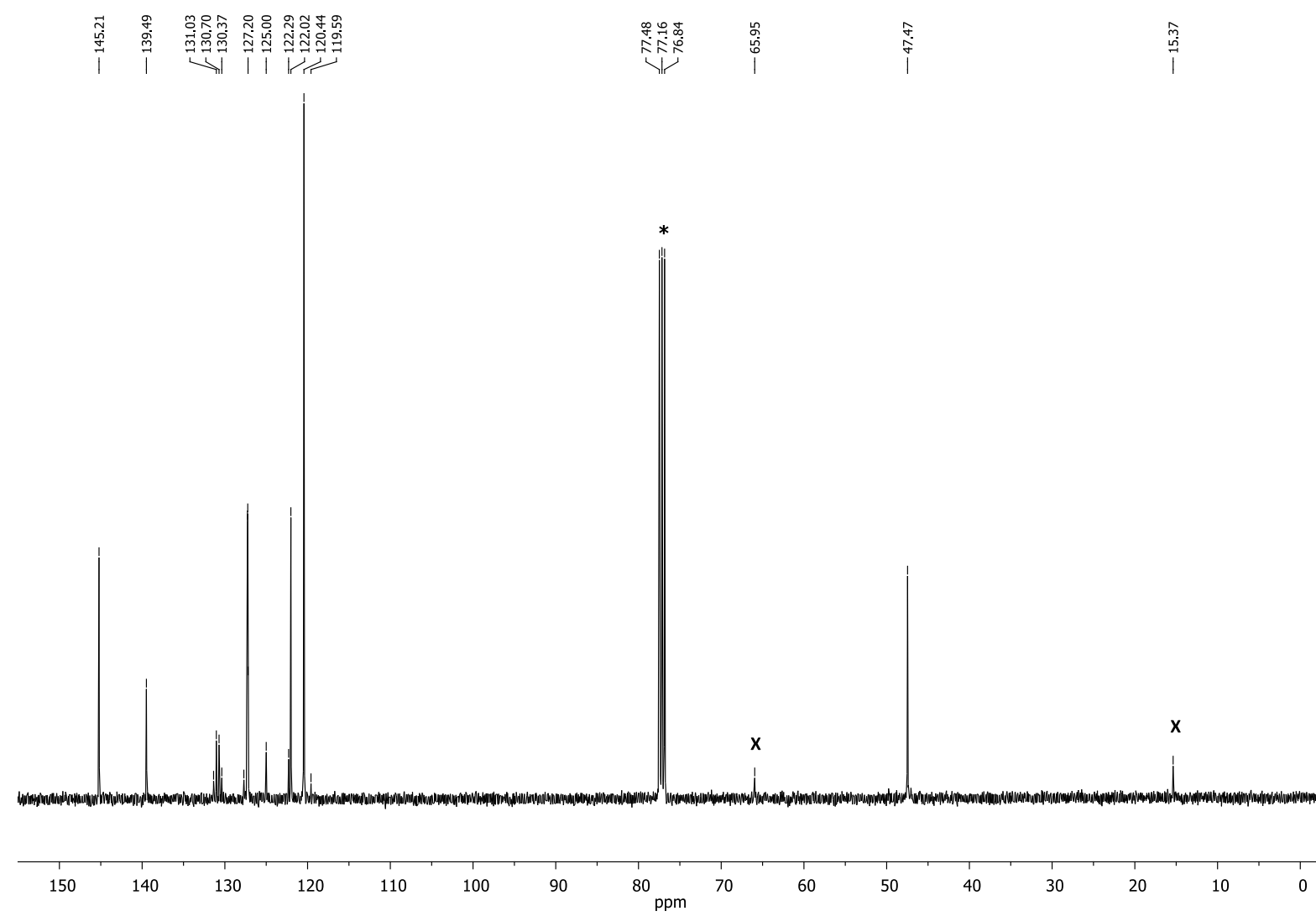

Figure S9. ${ }^{13} \mathrm{C}$ NMR spectrum of $\mathbf{3} \mathbf{b}$, recorded in $\mathrm{CDCl}_{3}$ solution $\left(*=\mathrm{CDCl}_{3} ; \mathbf{x}=\mathrm{Et}_{2} \mathrm{O}\right)$.

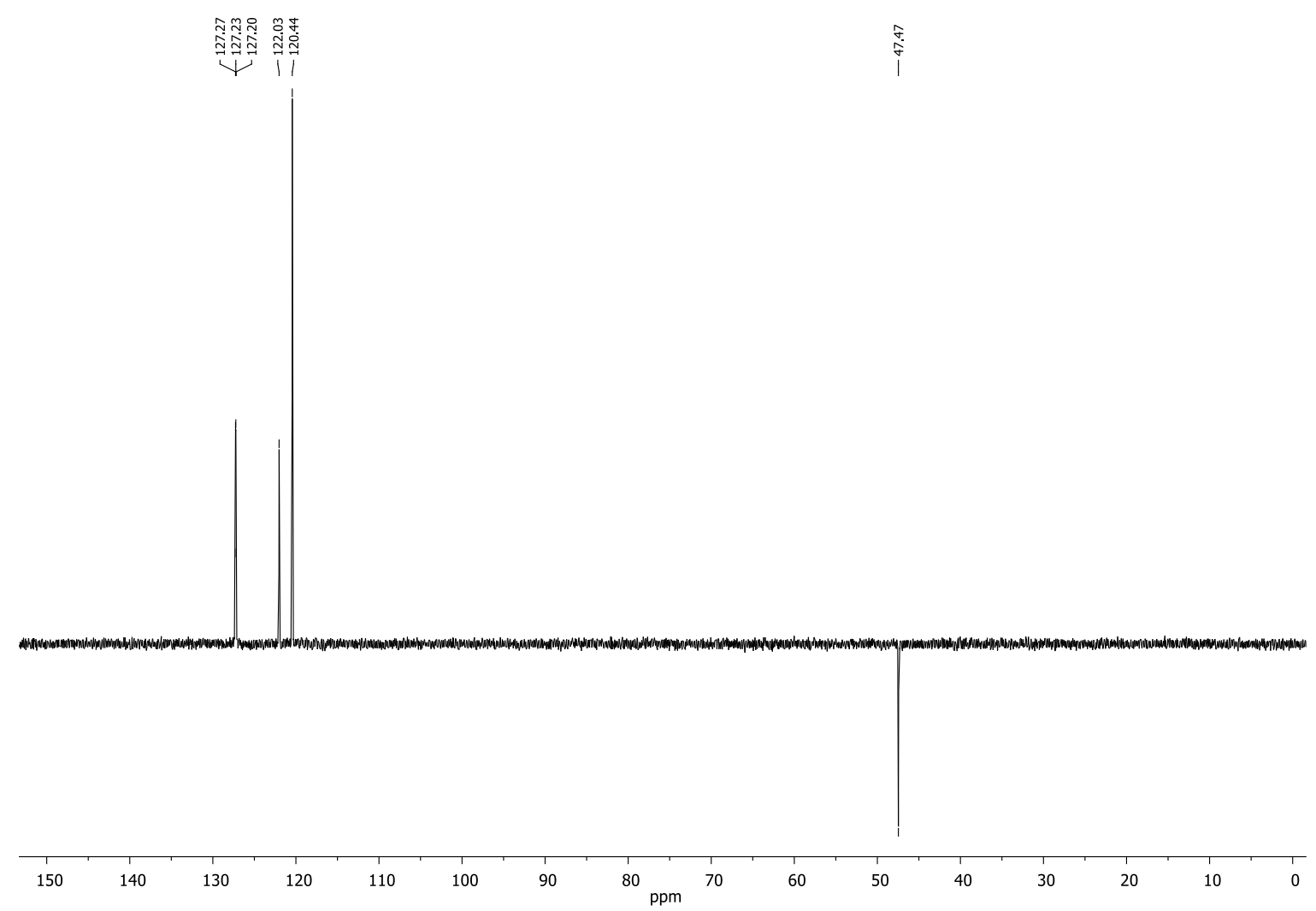

Figure S10. DEPT-135 NMR spectrum of $\mathbf{3 b}$, recorded in $\mathrm{CDCl}_{3}$ solution. 


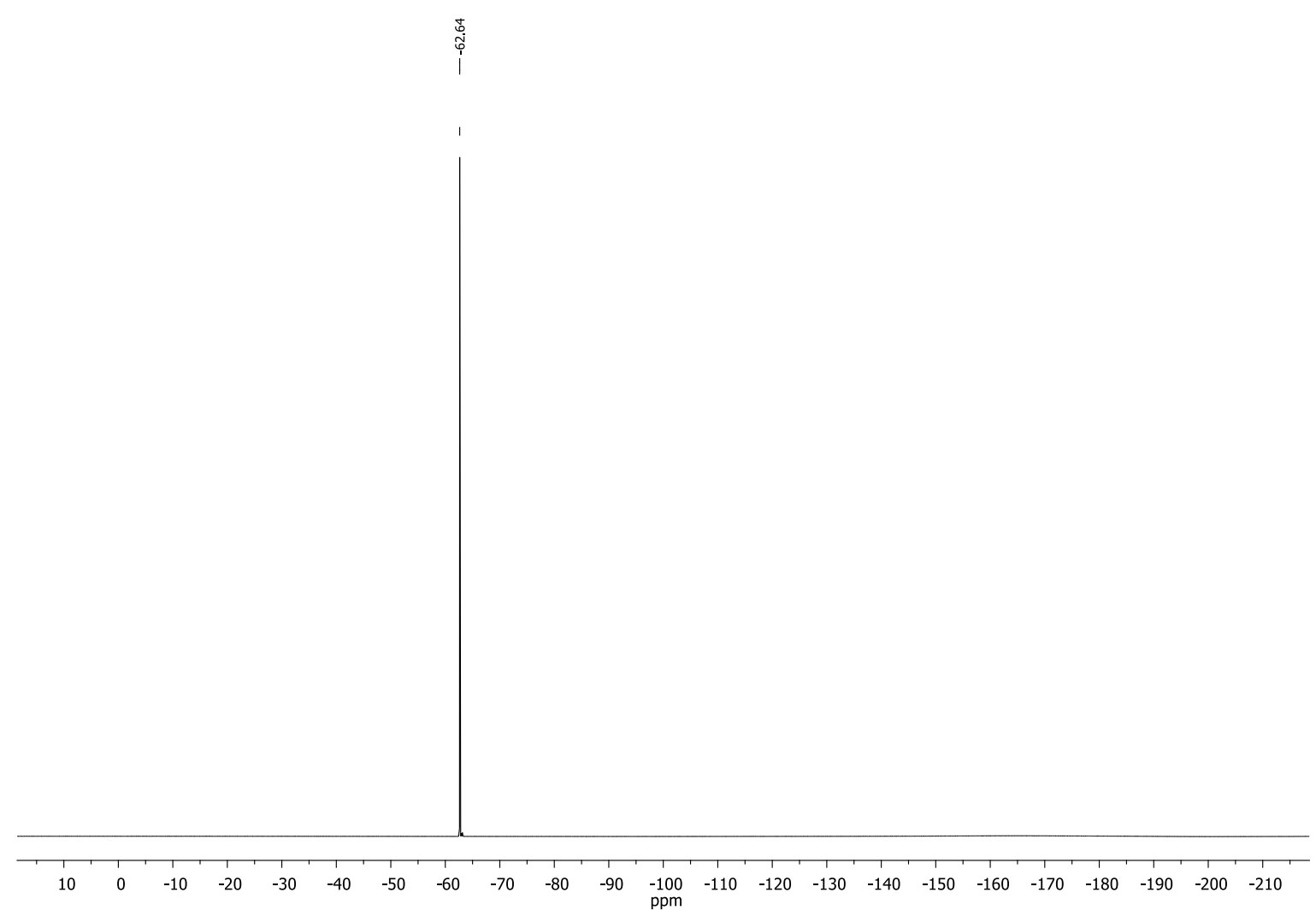

Figure $\mathbf{S 1 1 .}{ }^{19} \mathrm{~F}$ NMR spectrum of $\mathbf{3 b}$, recorded in $\mathrm{CDCl}_{3}$ solution.

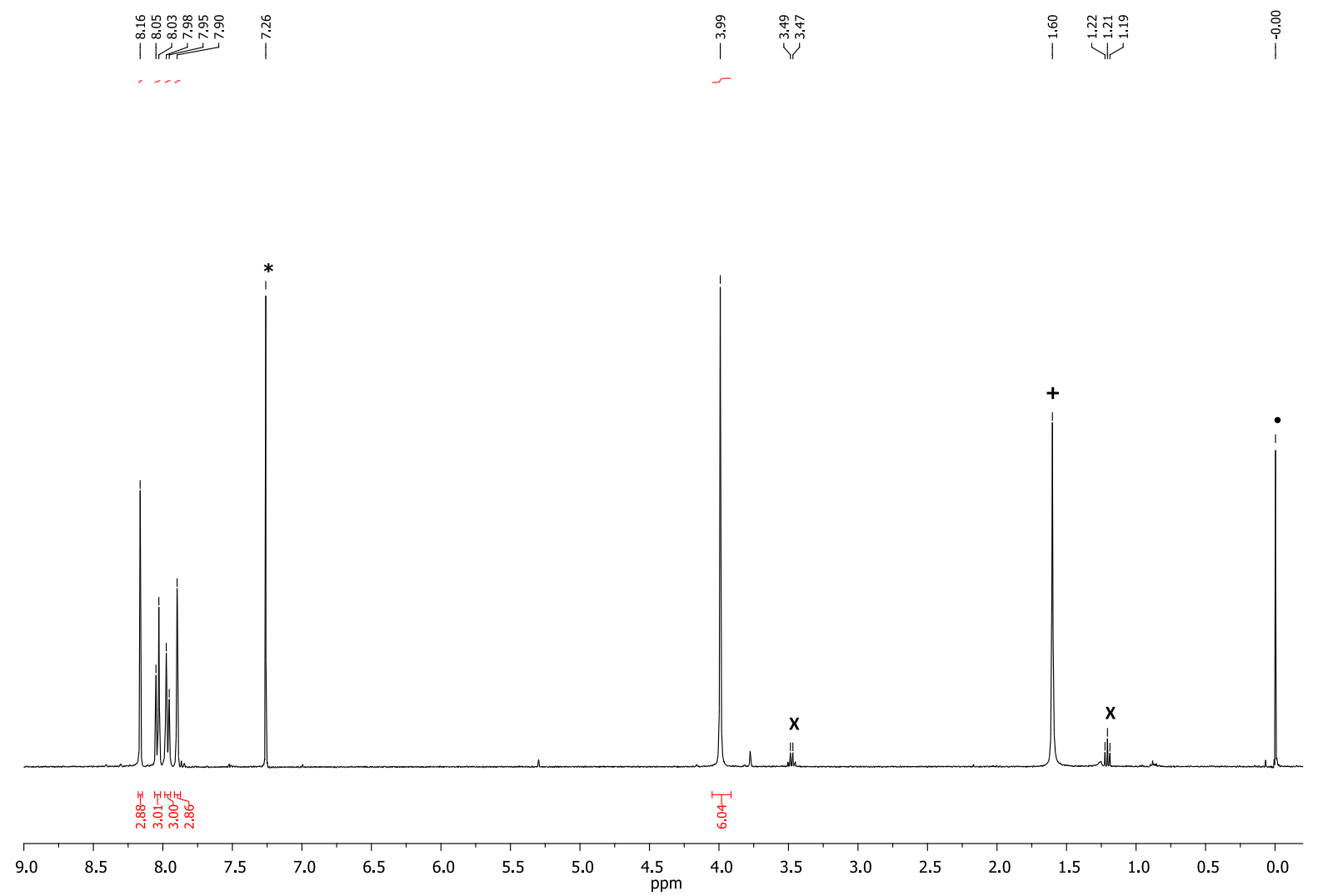

Figure S12. ${ }^{1} \mathrm{H}$ NMR spectrum of $3 \mathbf{c}$, recorded in $\mathrm{CDCl}_{3}$ solution $\left({ }^{*}=\mathrm{CDCl}_{3} ;+=\mathrm{H}_{2} \mathrm{O} ; \bullet=\mathrm{TMS} \mathbf{x}=\mathrm{Et}_{2} \mathrm{O}\right)$. 


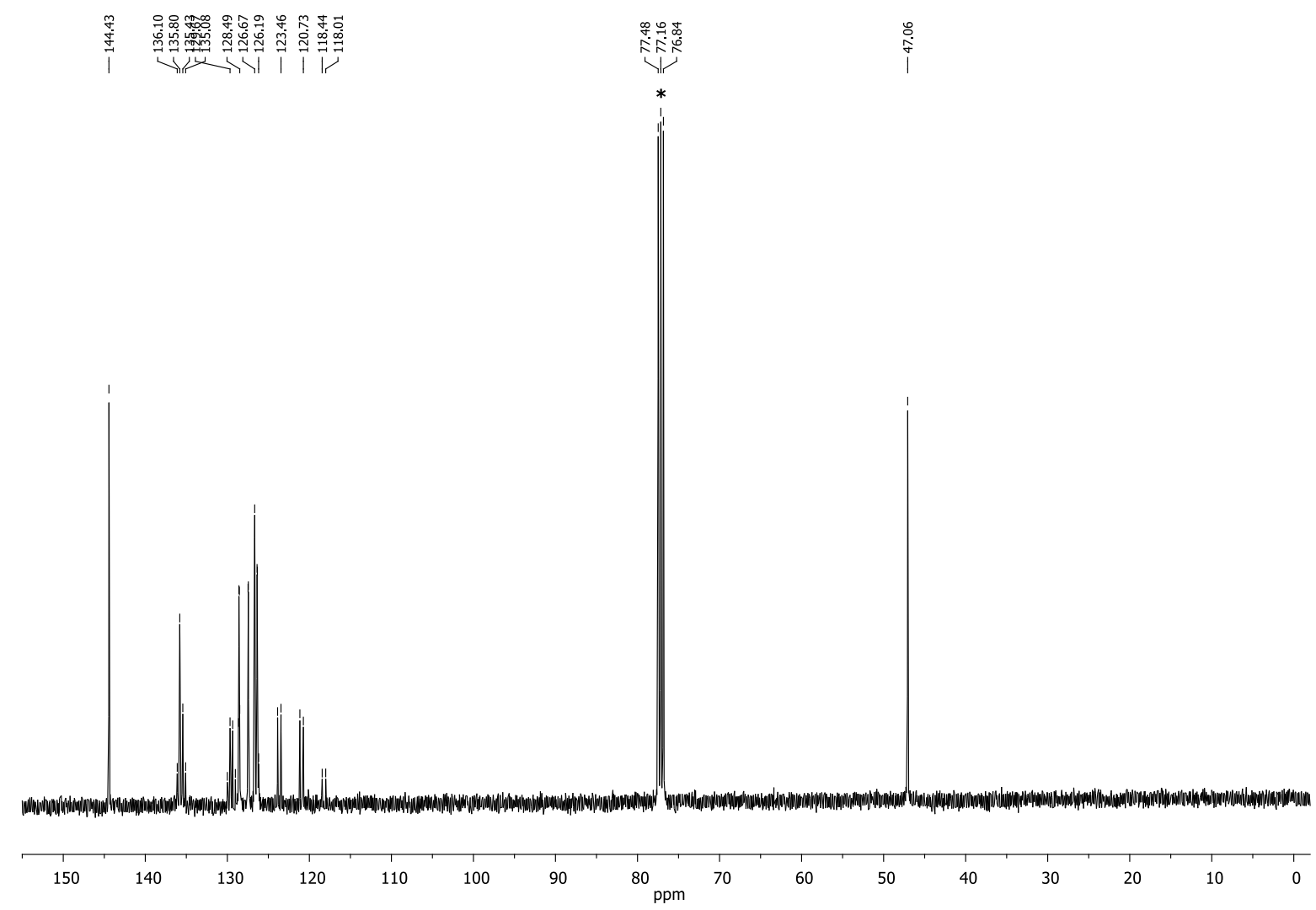

Figure S13. ${ }^{13} \mathrm{C}$ NMR spectrum of $3 \mathrm{c}$, recorded in $\mathrm{CDCl}_{3}\left({ }^{*}\right)$ solution.

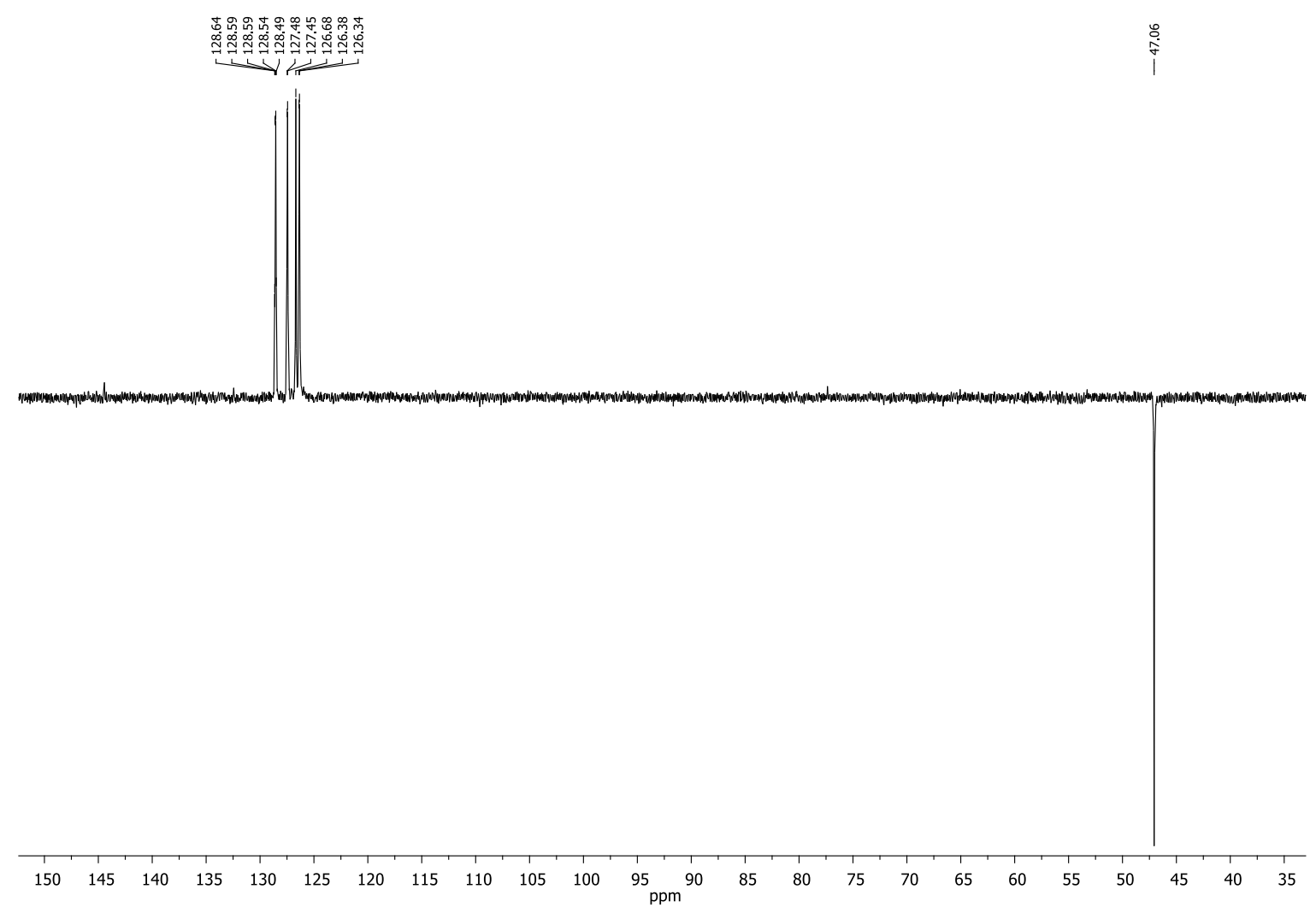

Figure S14. DEPT-135 NMR spectrum of $\mathbf{3 c}$, recorded in $\mathrm{CDCl}_{3}$ solution. 


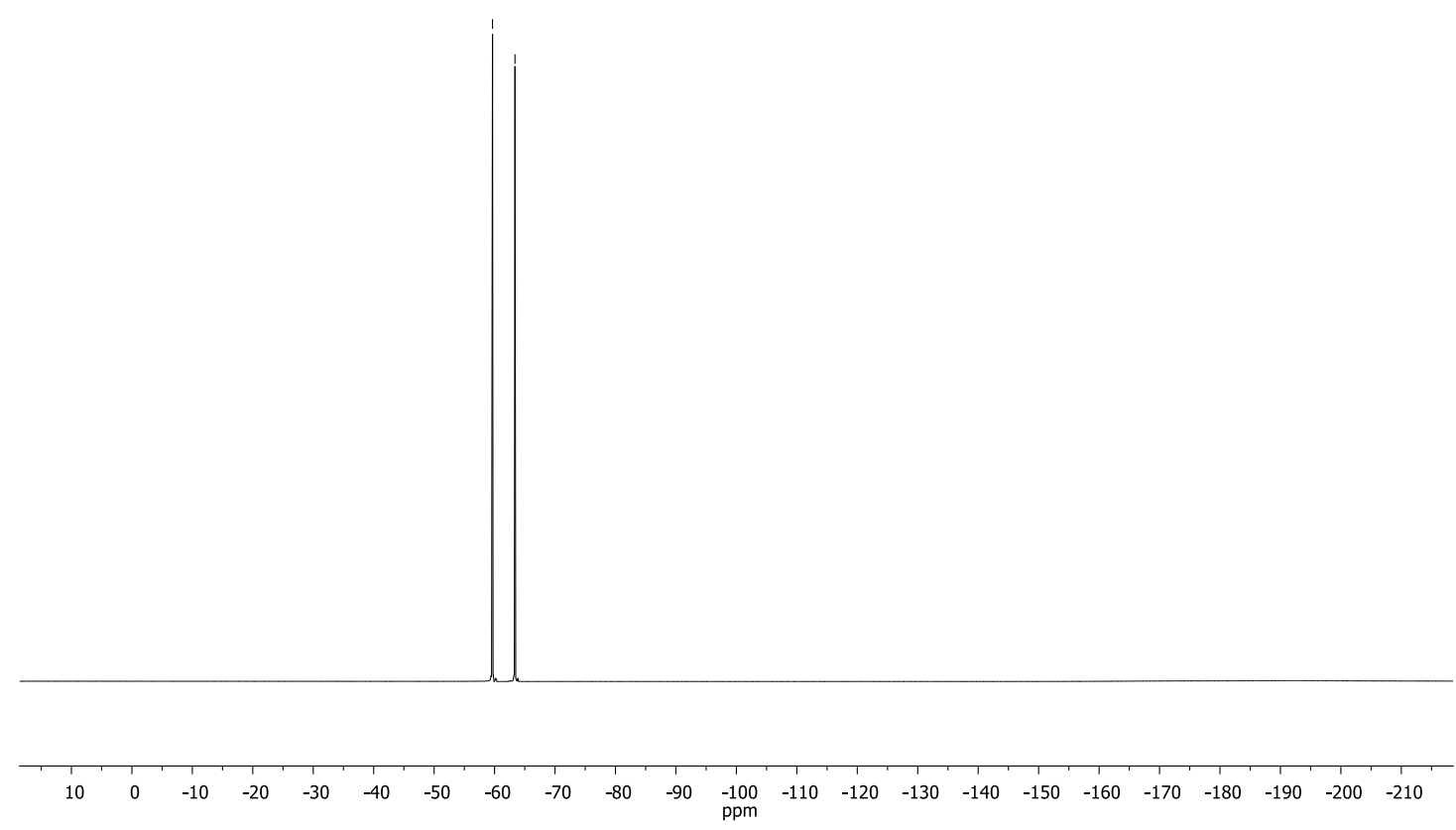

Figure S15. ${ }^{19} \mathrm{~F}$ NMR spectrum of 3c, recorded in $\mathrm{CDCl}_{3}$ solution.

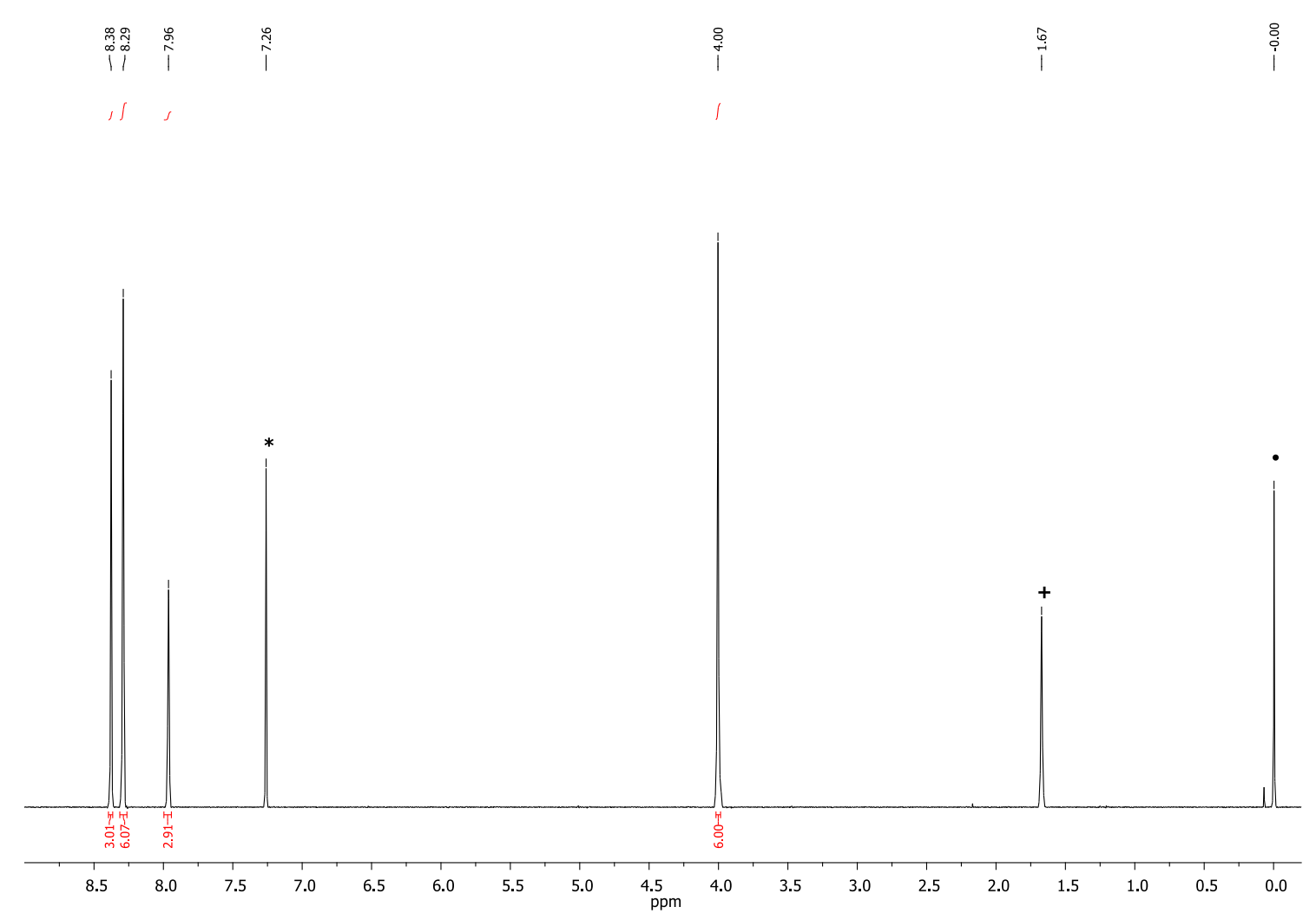

Figure S16. ${ }^{1} \mathrm{H} \mathrm{NMR}$ spectrum of $3 \mathrm{~d}$, recorded in $\mathrm{CDCl}_{3}$ solution $\left(*=\mathrm{CDCl}_{3} ;+=\mathrm{H}_{2} \mathrm{O} ; \bullet=\mathrm{TMS}\right)$. 


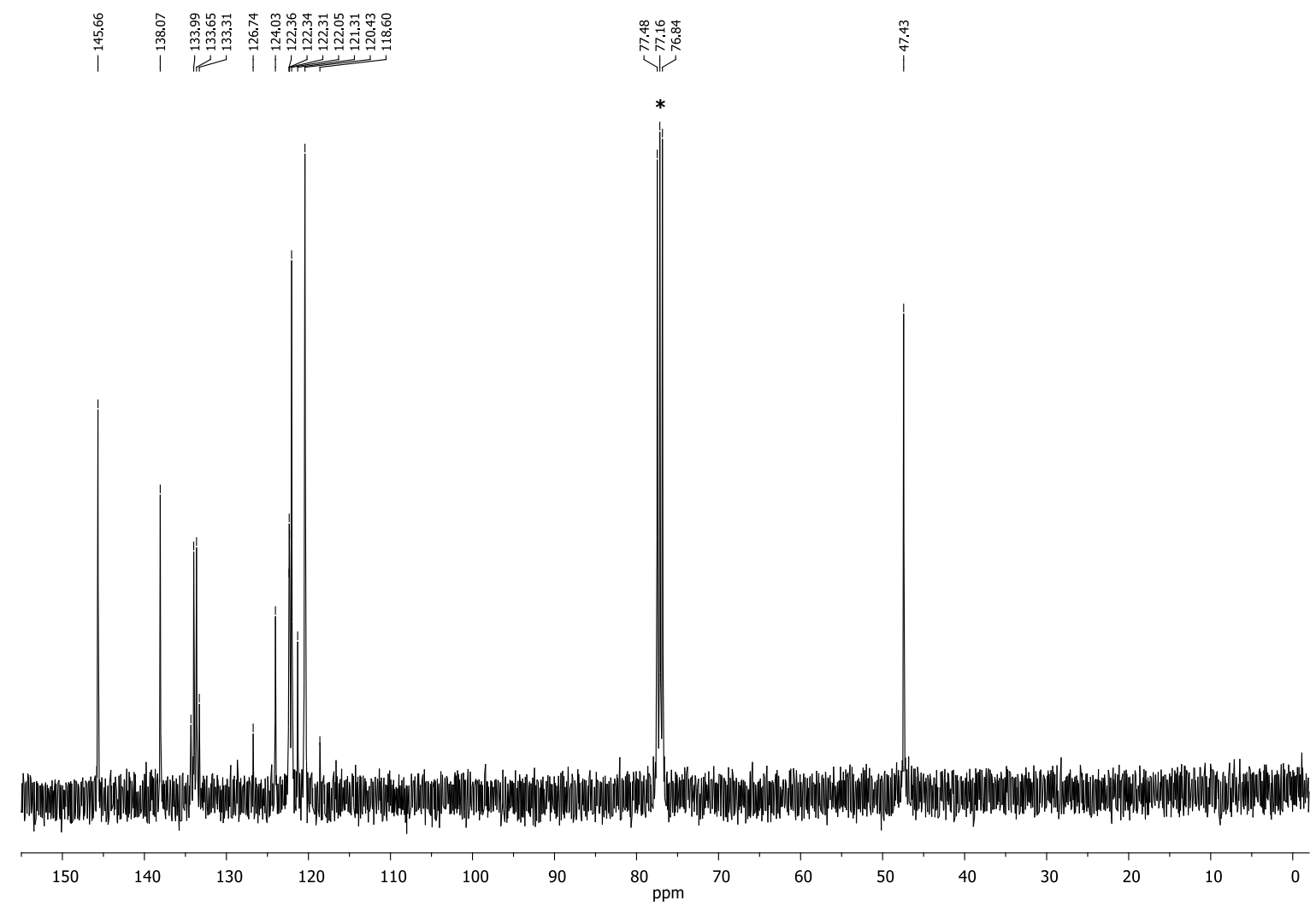

Figure S17. ${ }^{13} \mathrm{C}$ NMR spectrum of $\mathbf{3 d}$, recorded in $\mathrm{CDCl}_{3}\left({ }^{*}\right)$ solution.

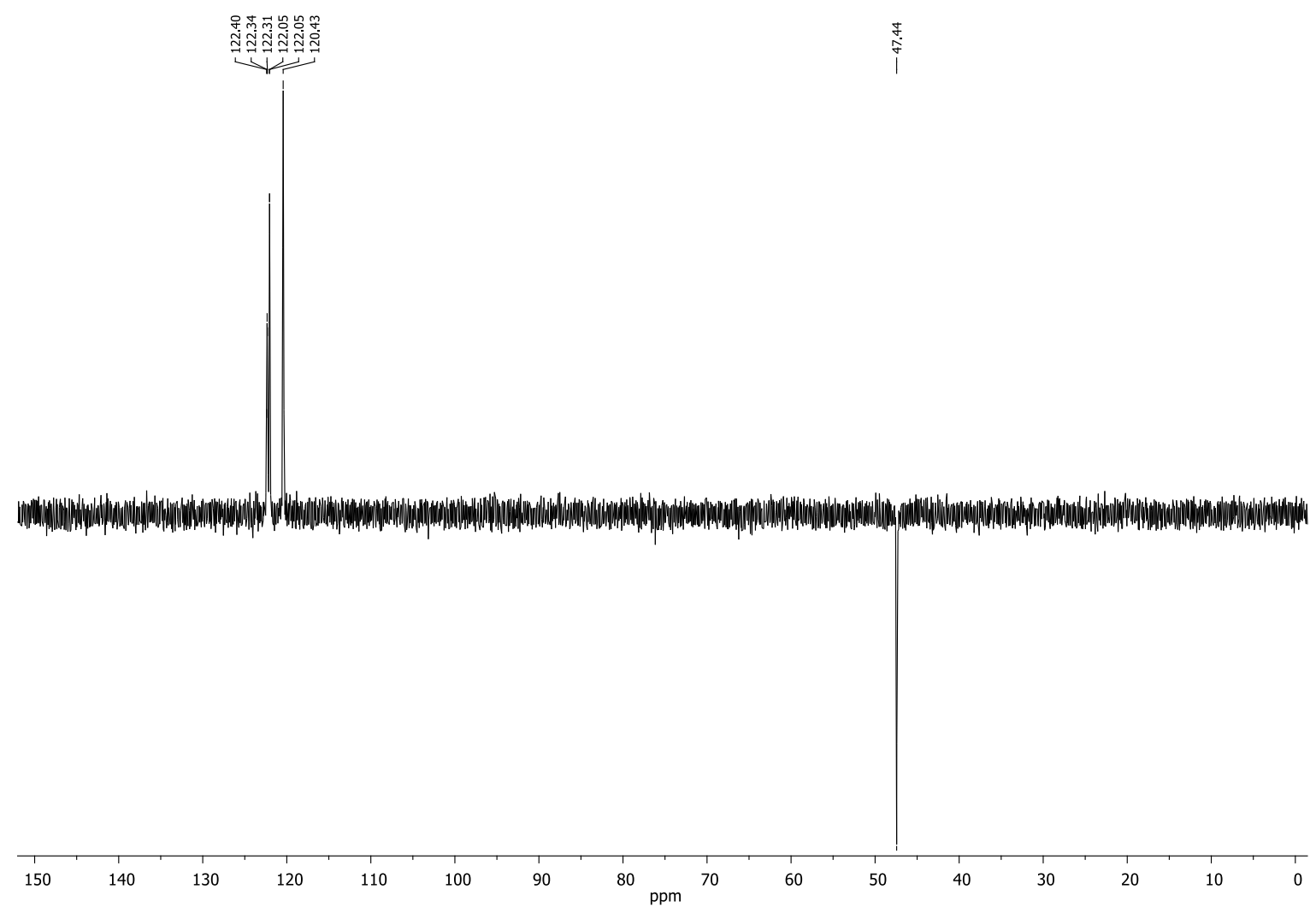

Figure S18. DEPT-135 NMR spectrum of $\mathbf{3 d}$, recorded in $\mathrm{CDCl}_{3}$ solution. 


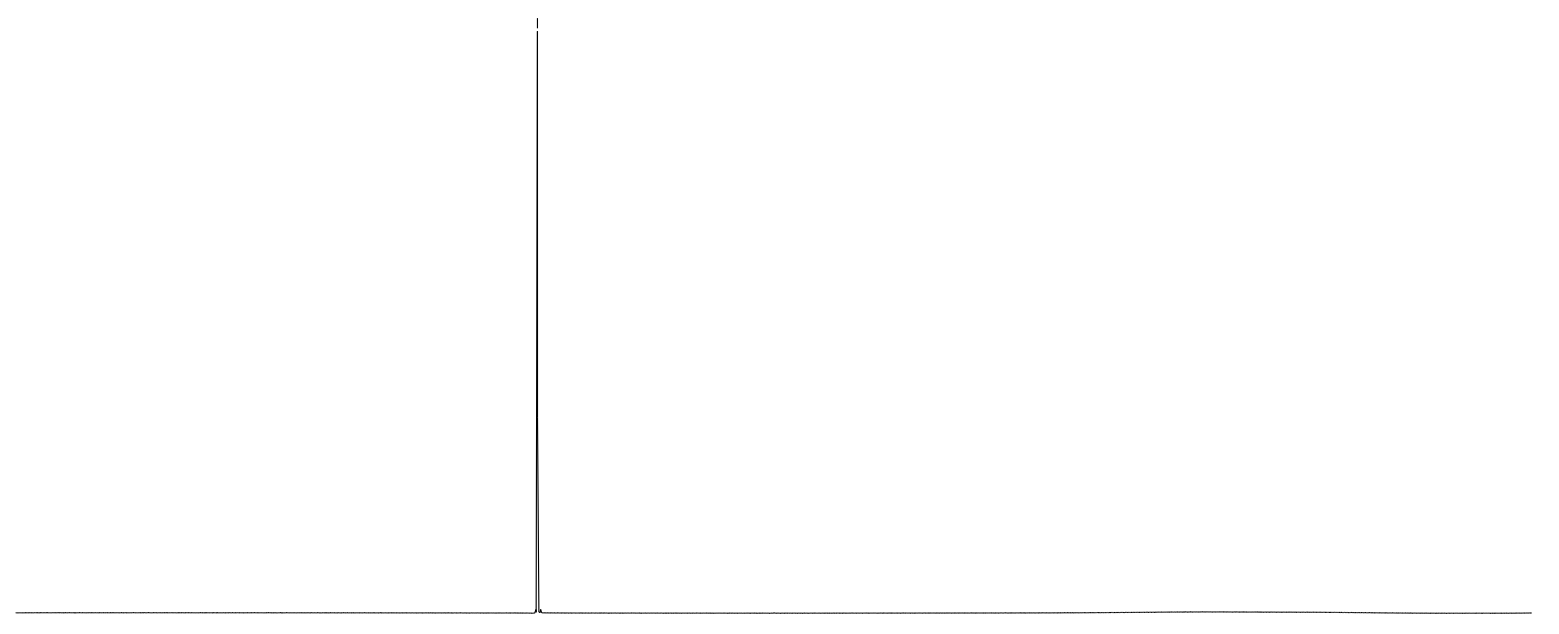

\begin{tabular}{llllllllllllllllllllllll}
\hline 10 & 0 & -10 & -20 & -30 & -40 & -50 & -60 & -70 & -80 & -90 & -100 & -110 & -120 & -130 & -140 & -150 & -160 & -170 & -180 & -190 & -200 & -210 & 0
\end{tabular}

Figure S19. ${ }^{19} \mathrm{~F}$ NMR spectrum of $\mathbf{3 d}$, recorded in $\mathrm{CDCl}_{3}$ solution.

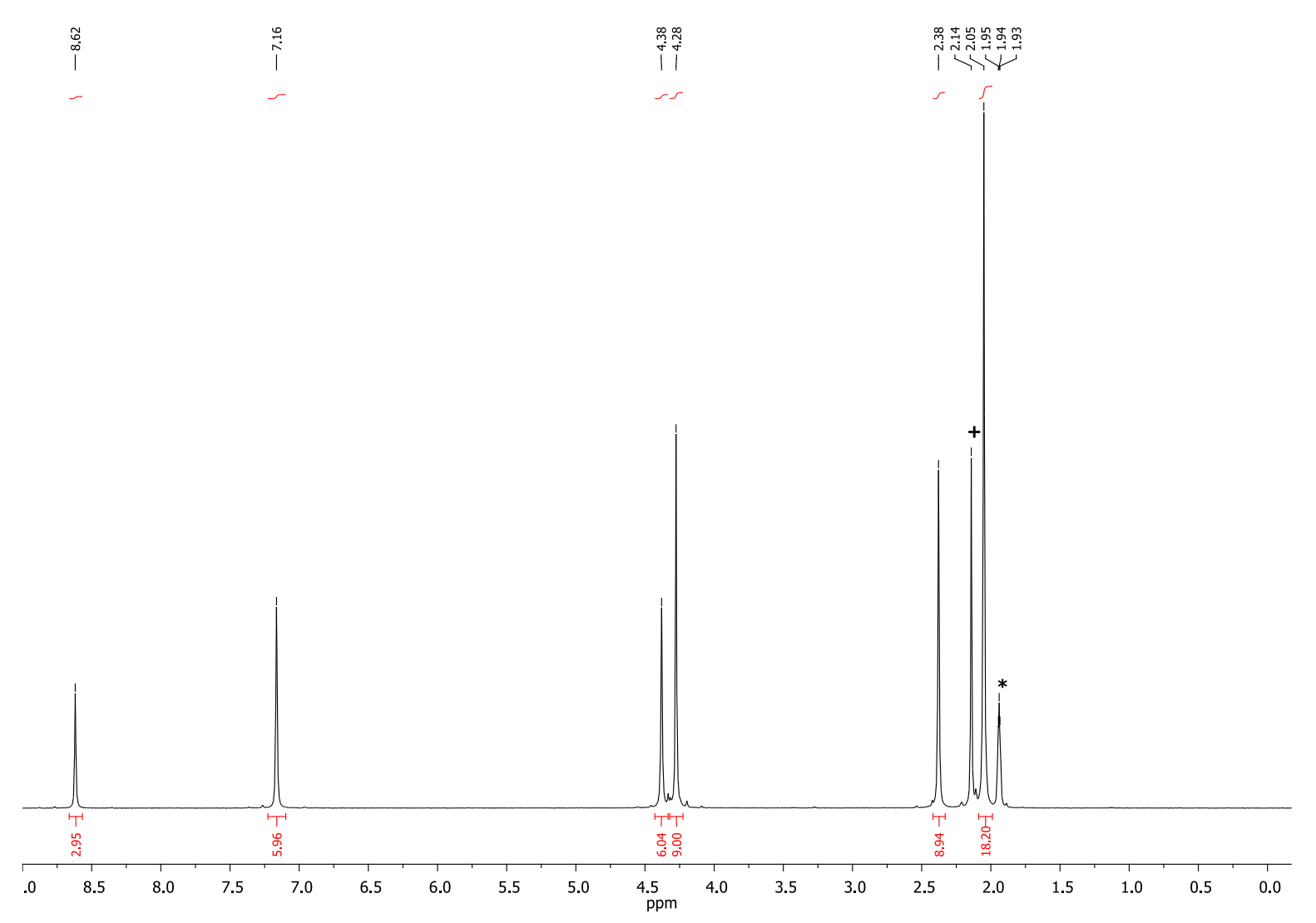

Figure S20. ${ }^{1} \mathrm{H}$ NMR spectrum of $[4 \mathrm{a}]\left(\mathrm{BF}_{4}\right)_{3}$, recorded in $\mathrm{CD}_{3} \mathrm{CN}$ solution $\left(*=\mathrm{CD}_{3} \mathrm{CN} ;+=\mathrm{H}_{2} \mathrm{O}\right)$. 


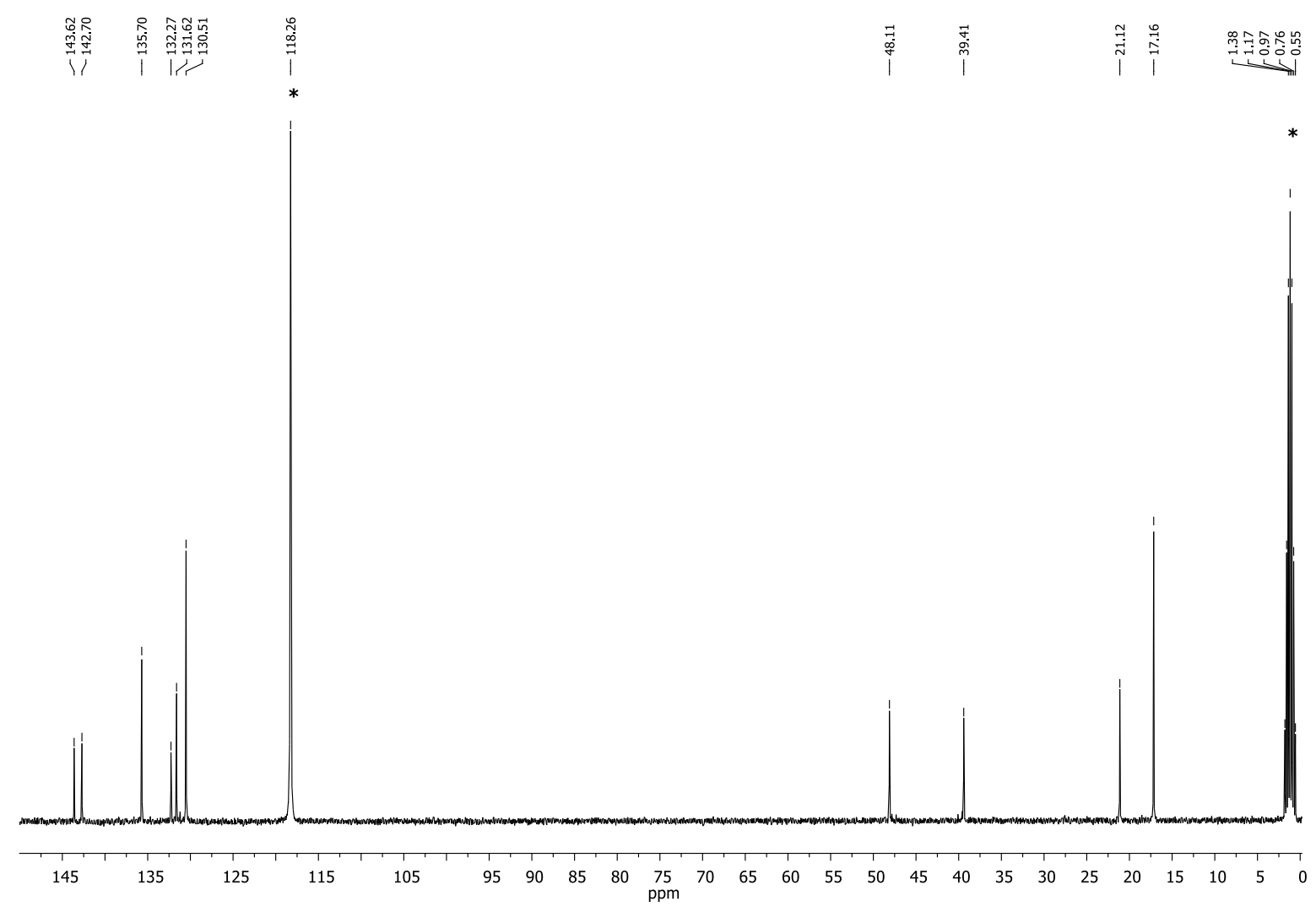

Figure S21. ${ }^{13} \mathrm{C}$ NMR spectrum of $[4 \mathrm{a}]\left(\mathrm{BF}_{4}\right)_{3}$, recorded in $\mathrm{CD}_{3} \mathrm{CN}\left({ }^{*}\right)$ solution.

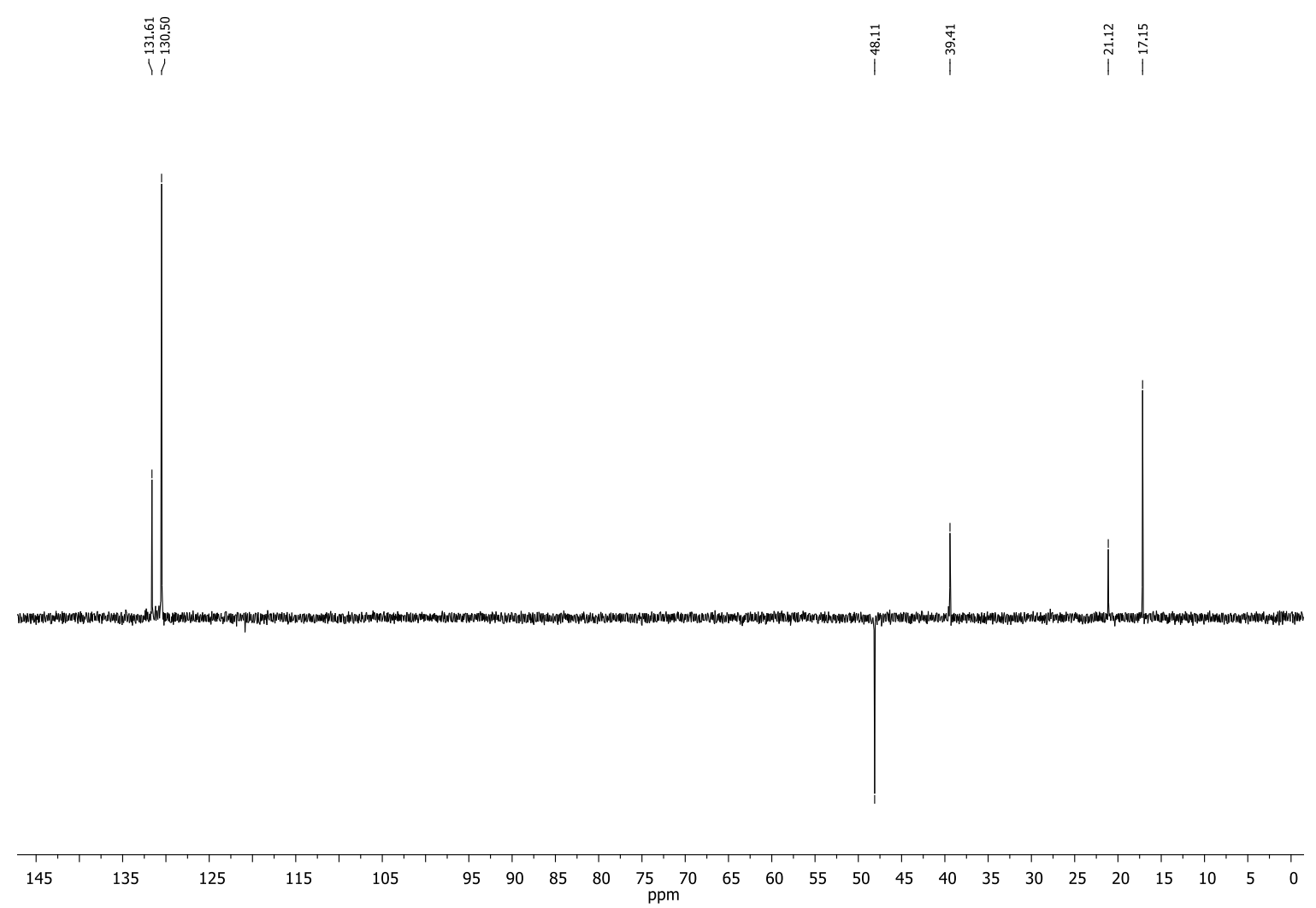

Figure S22. DEPT-135 NMR spectrum of $[4 \mathrm{a}]\left(\mathrm{BF}_{4}\right)_{3}$, recorded in $\mathrm{CD}_{3} \mathrm{CN}$ solution. 


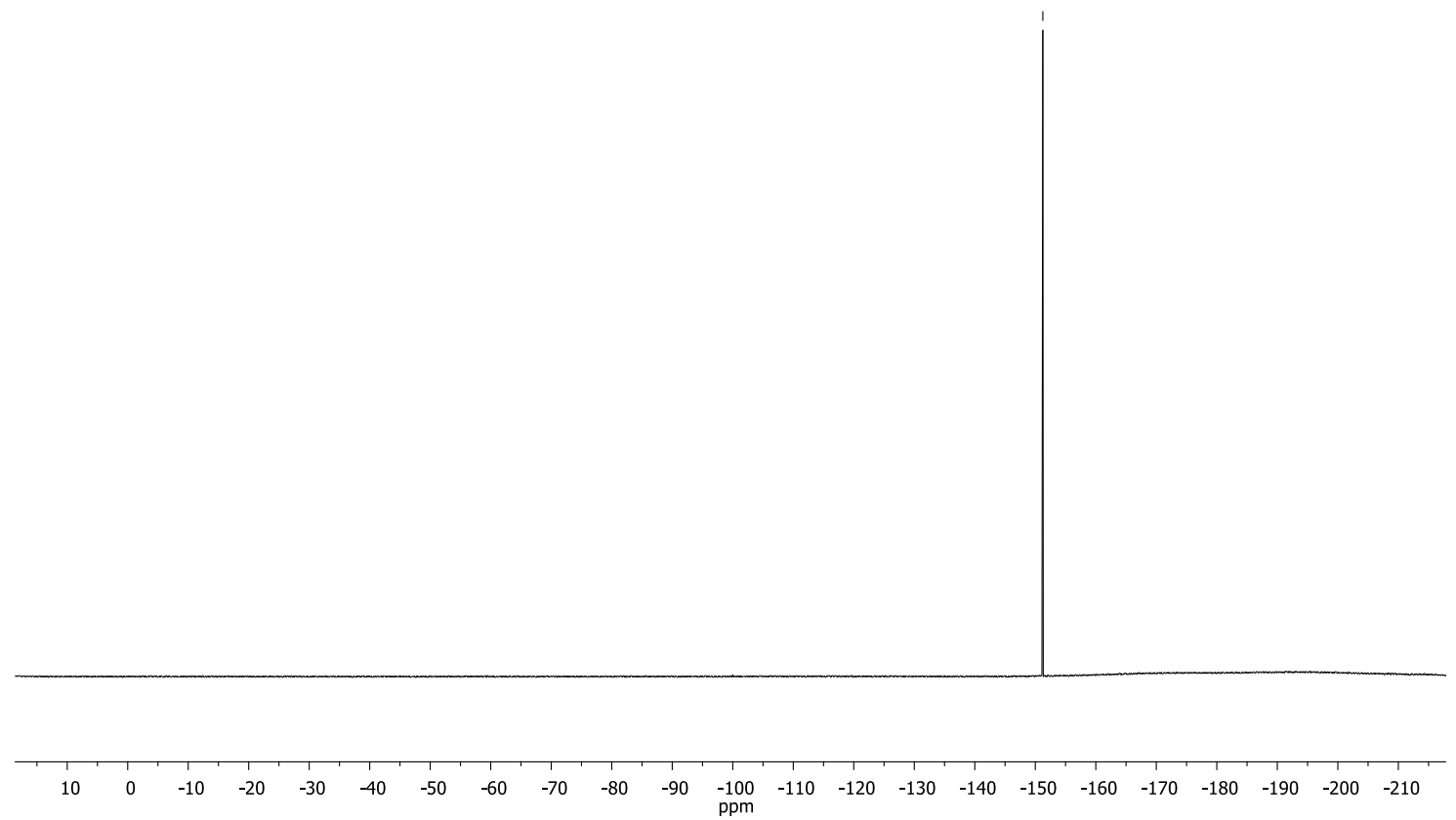

Figure S23. ${ }^{19} \mathrm{~F}$ NMR NMR spectrum of $[4 \mathrm{a}]\left(\mathrm{BF}_{4}\right)_{3}$, recorded in $\mathrm{CD}_{3} \mathrm{CN}$ solution.

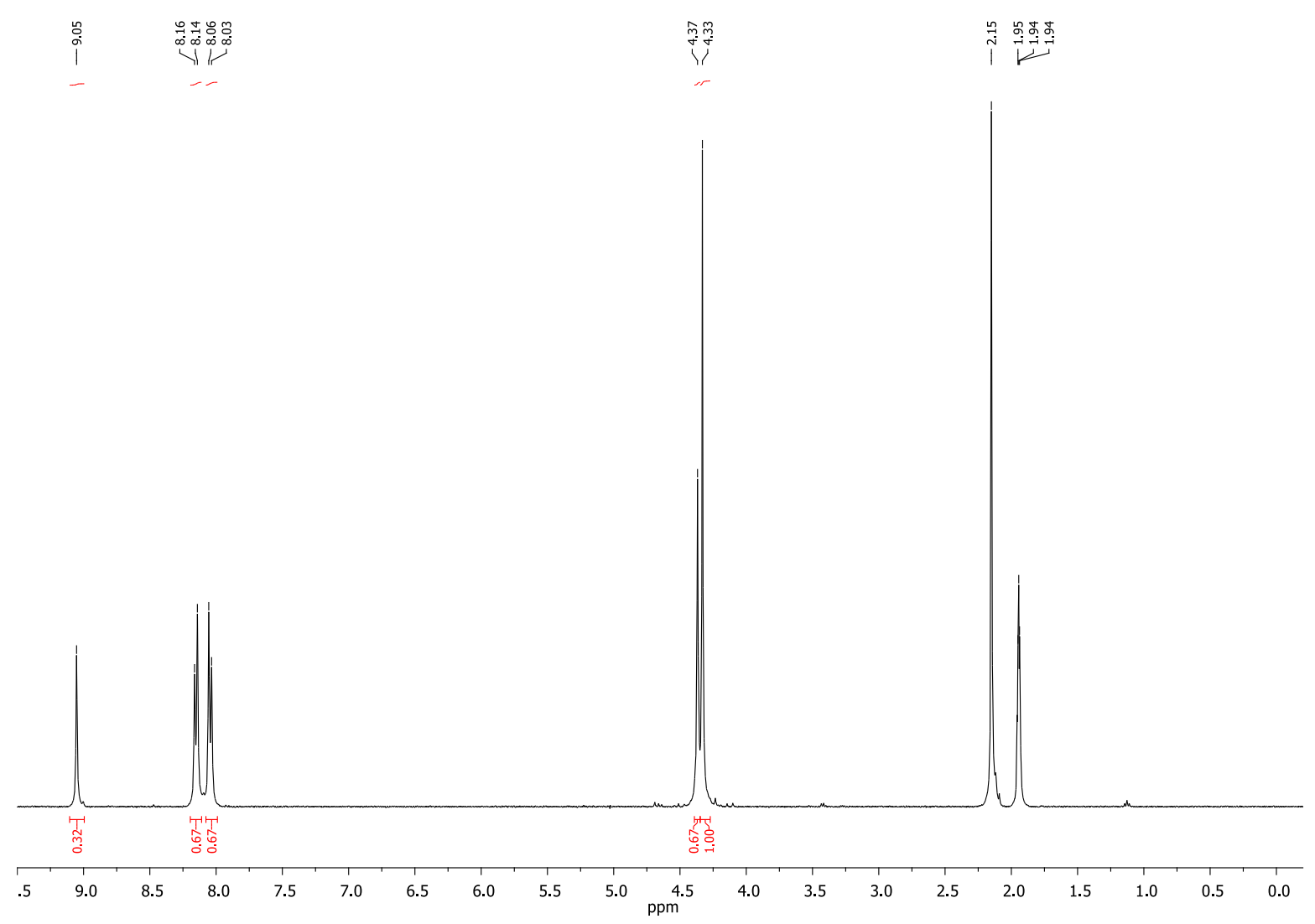

Figure S24. ${ }^{1} \mathrm{H}$ NMR spectrum of $[4 \mathbf{b}]\left(\mathrm{BF}_{4}\right)_{3}$, recorded in $\mathrm{CD}_{3} \mathrm{CN}$ solution $\left({ }^{*}=\mathrm{CD}_{3} \mathrm{CN} ;+=\mathrm{H}_{2} \mathrm{O}\right)$. 


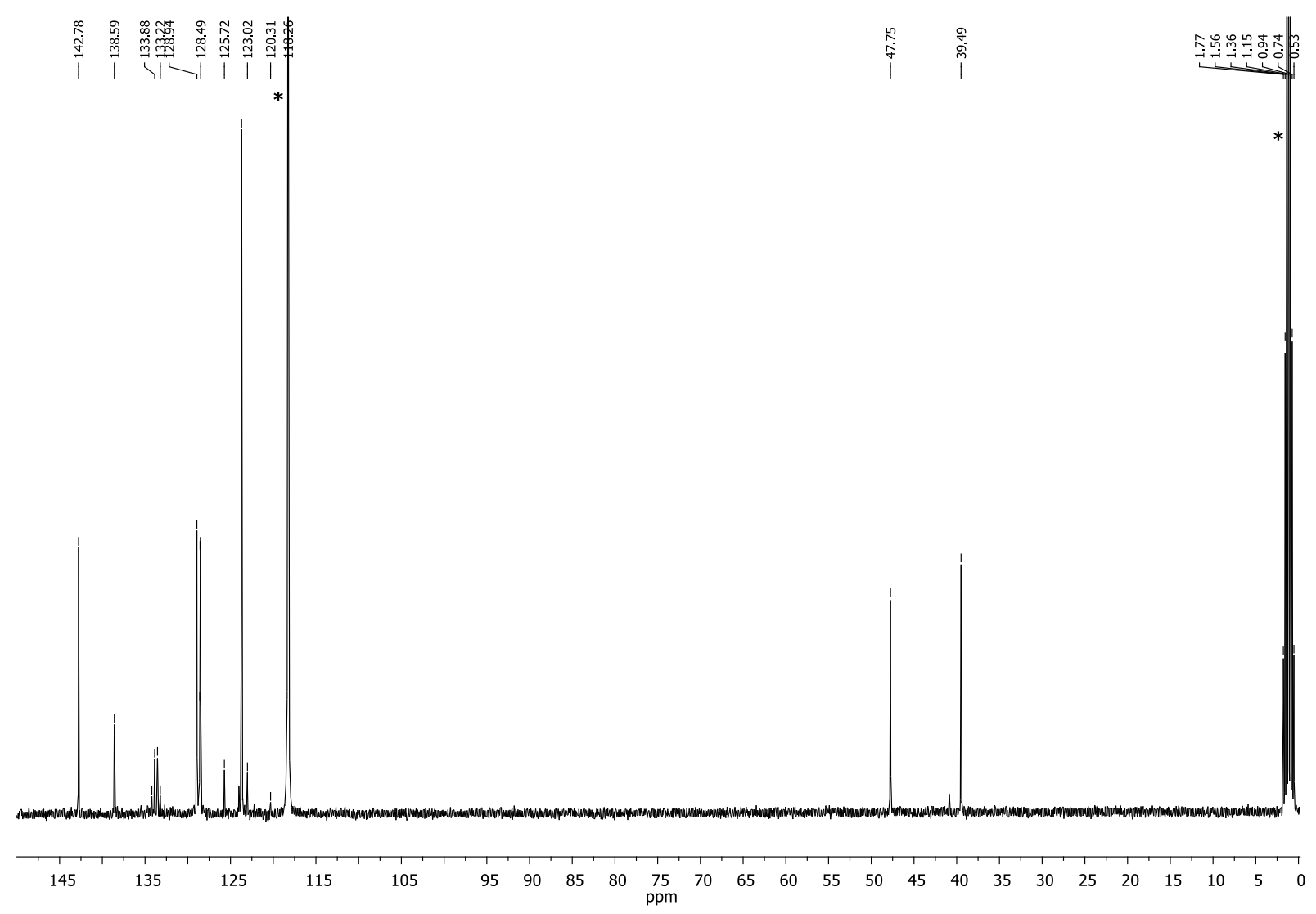

Figure S25. ${ }^{13} \mathrm{C}$ NMR spectrum of $[4 \mathbf{b}]\left(\mathrm{BF}_{4}\right)_{3}$, recorded in $\mathrm{CD}_{3} \mathrm{CN}\left({ }^{*}\right)$ solution.

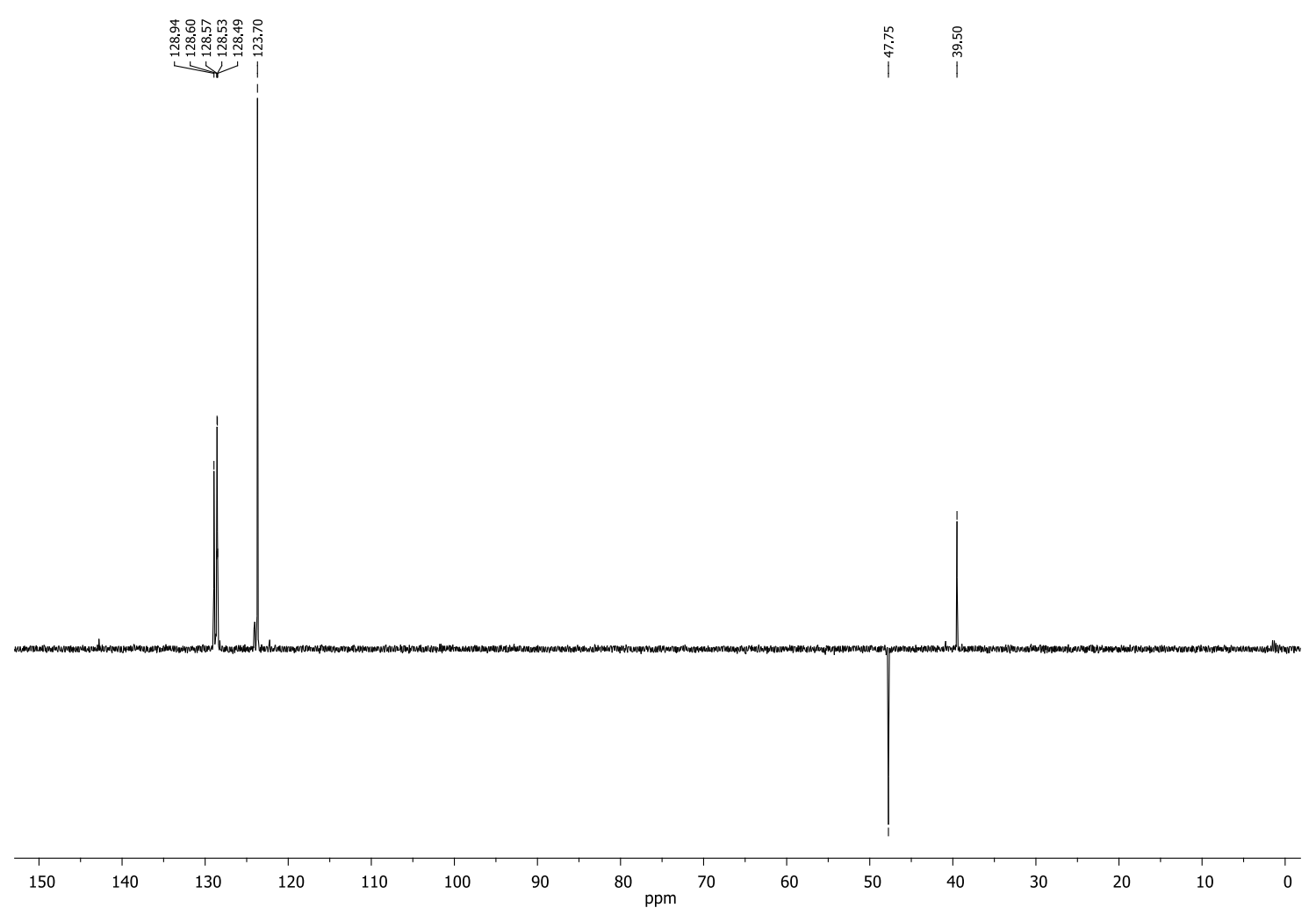

Figure S26. DEPT-135 NMR spectrum of $[4 \mathbf{b}]\left(\mathrm{BF}_{4}\right)_{3}$, recorded in $\mathrm{CD}_{3} \mathrm{CN}$ solution. 


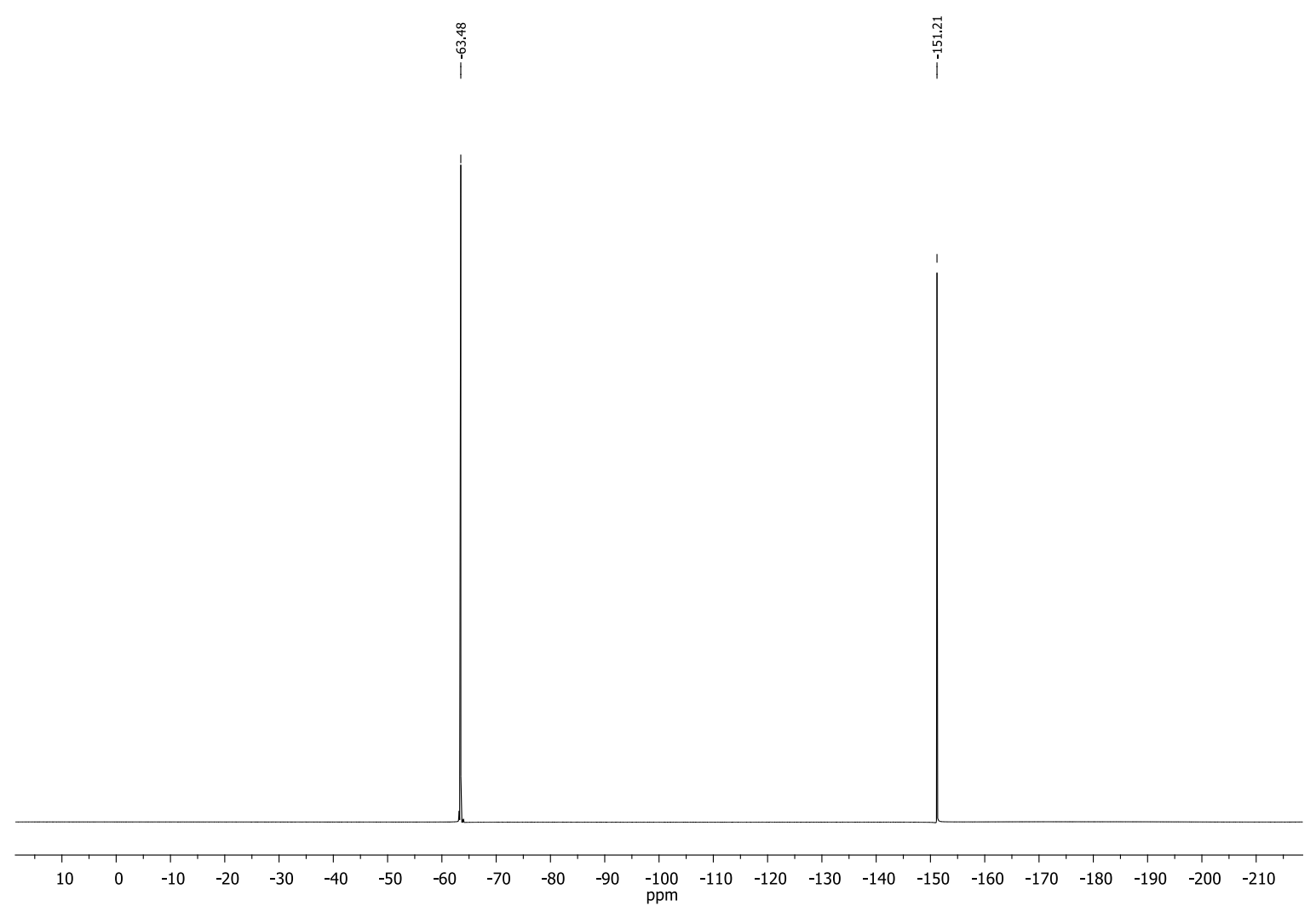

Figure S27. ${ }^{19} \mathrm{~F} N M R$ NMR spectrum of $[4 \mathbf{b}]\left(\mathrm{BF}_{4}\right)_{3}$, recorded in $\mathrm{CD}_{3} \mathrm{CN}$ solution.

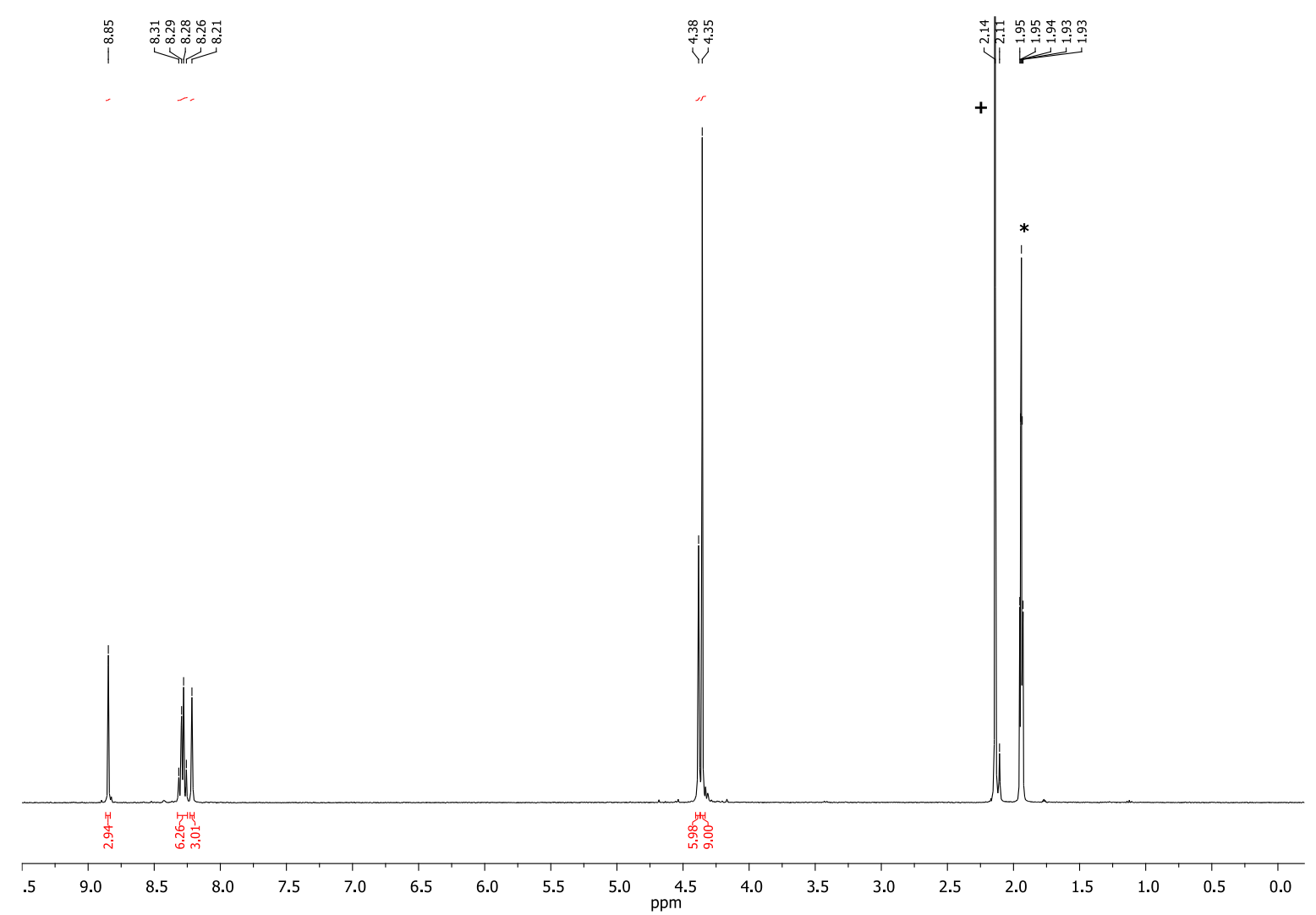

Figure S28. ${ }^{1} \mathrm{H}$ NMR spectrum of $[4 \mathrm{c}]\left(\mathrm{BF}_{4}\right)_{3}$, recorded in $\mathrm{CD}_{3} \mathrm{CN}$ solution $\left(*=\mathrm{CD}_{3} \mathrm{CN} ;+=\mathrm{H}_{2} \mathrm{O}\right)$. 


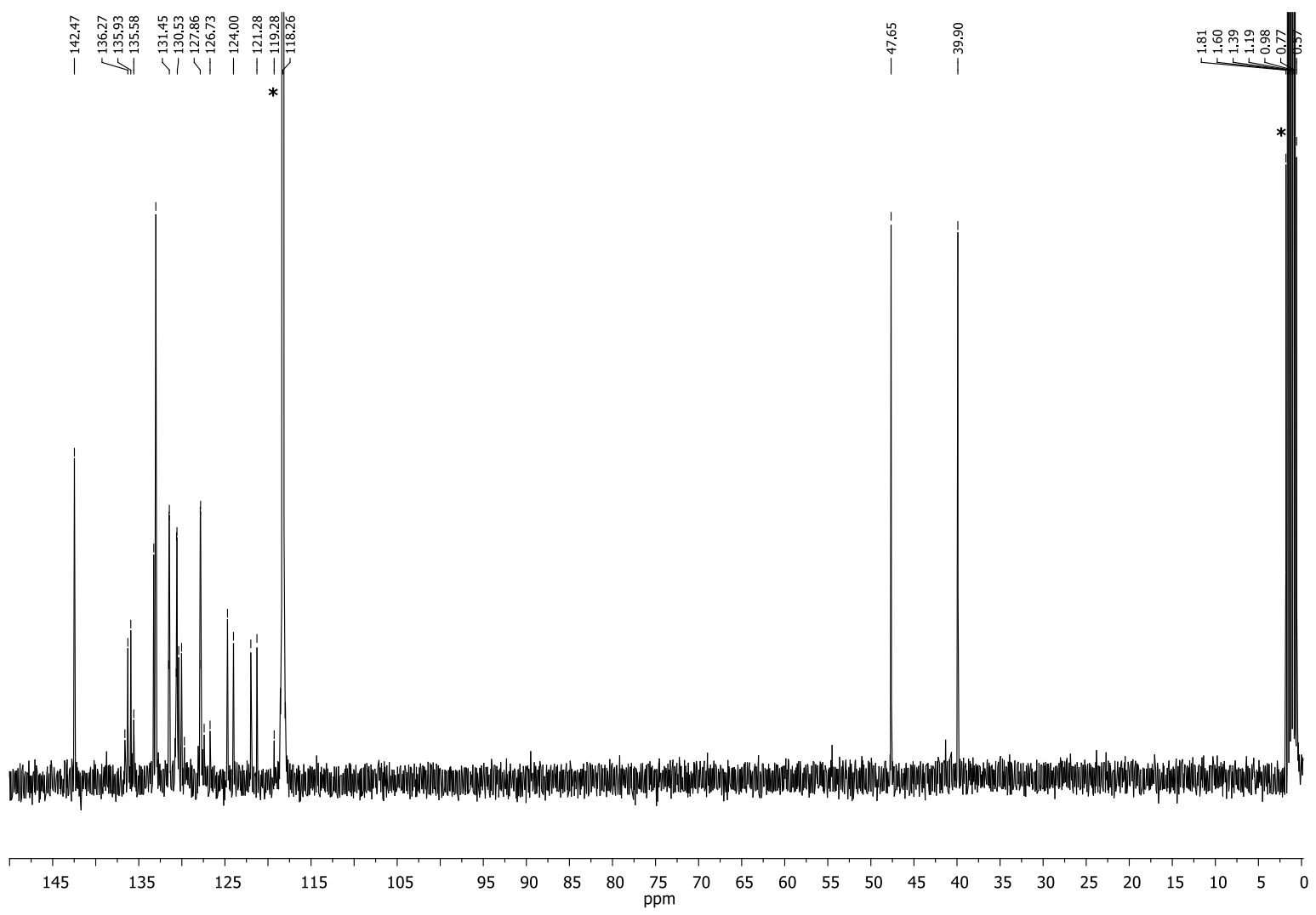

Figure S29. ${ }^{13} \mathrm{C}$ NMR spectrum of $[4 \mathrm{c}]\left(\mathrm{BF}_{4}\right)_{3}$, recorded in $\mathrm{CD}_{3} \mathrm{CN}\left({ }^{*}\right)$ solution.

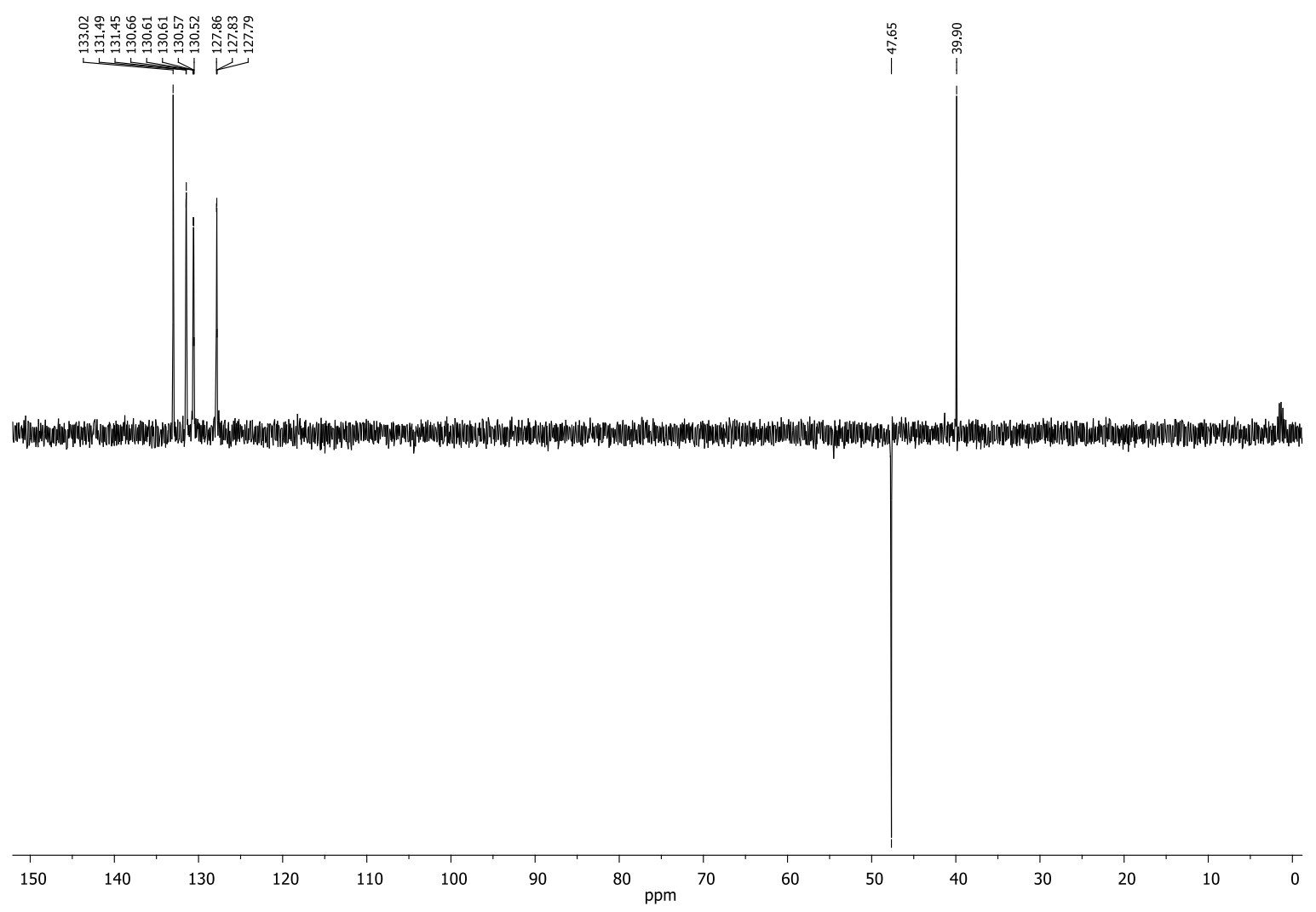

Figure S30. DEPT-135 NMR spectrum of $[4 \mathrm{c}]\left(\mathrm{BF}_{4}\right)_{3}$, recorded in $\mathrm{CD}_{3} \mathrm{CN}$ solution. 


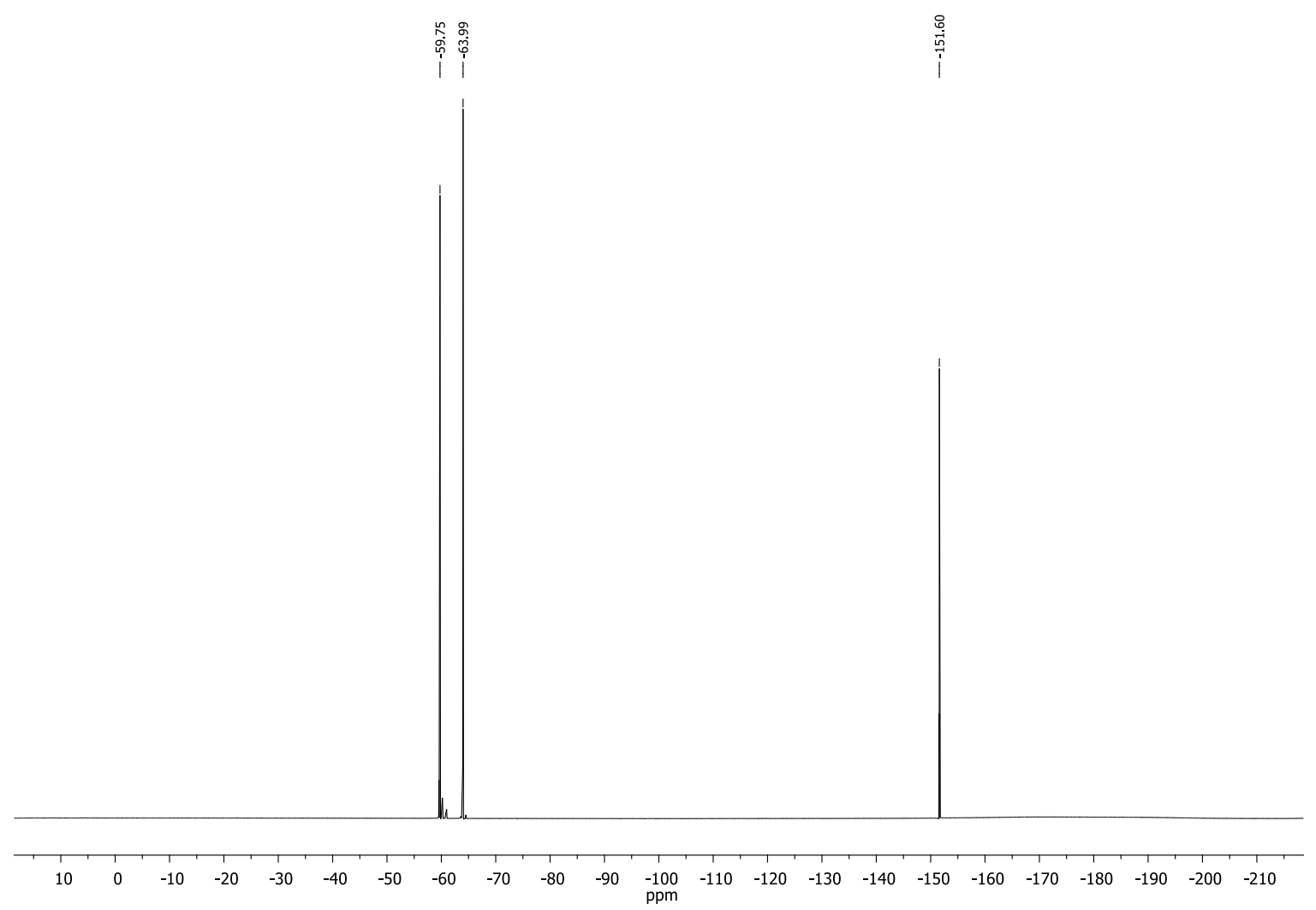

Figure S31. ${ }^{19} \mathrm{~F} N M R$ spectrum of $[4 \mathrm{c}]\left(\mathrm{BF}_{4}\right)_{3}$, recorded in $\mathrm{CD}_{3} \mathrm{CN}$ solution.

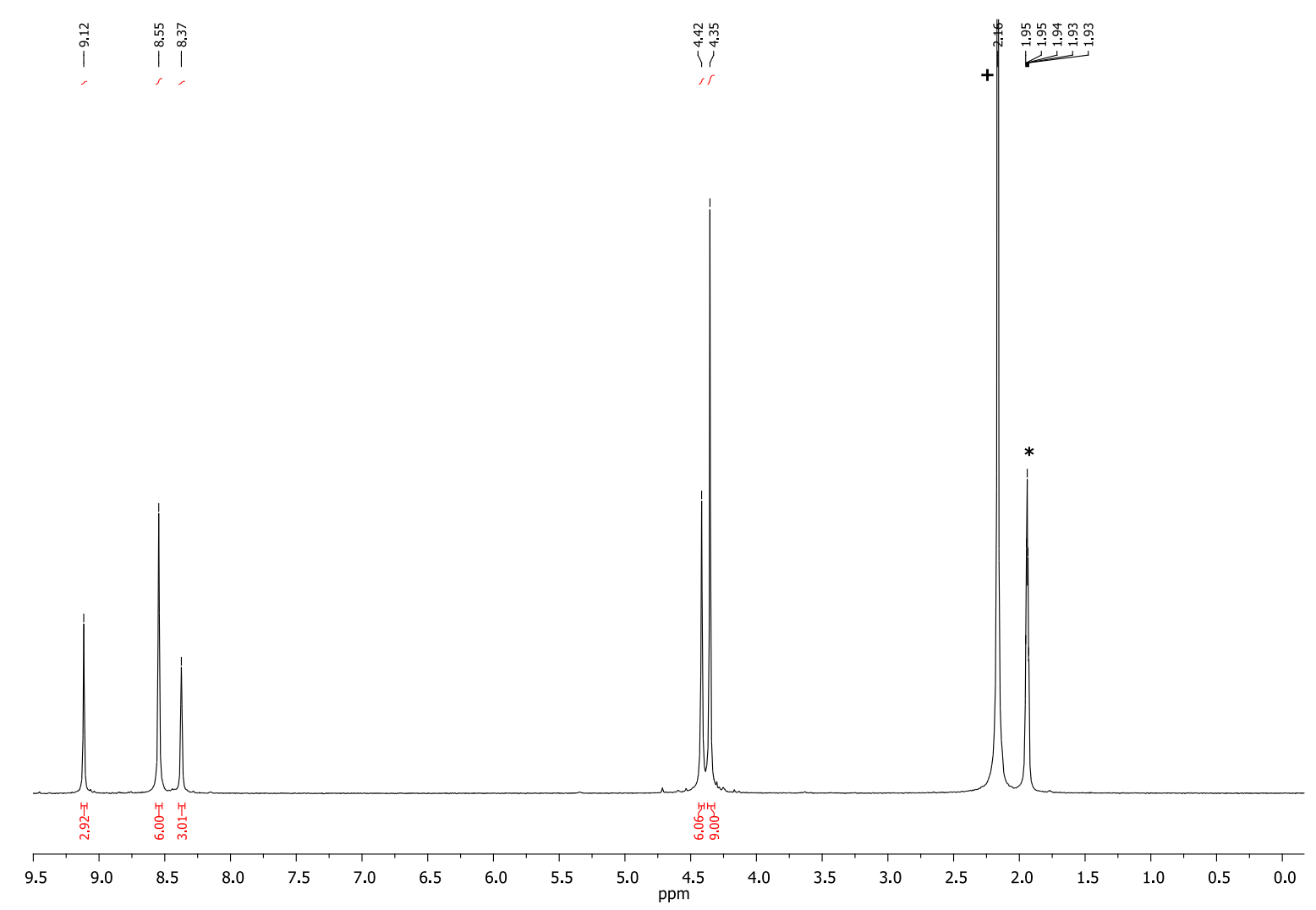

Figure S32. ${ }^{1} \mathrm{H}$ NMR spectrum of $[4 \mathrm{~d}]\left(\mathrm{BF}_{4}\right)_{3}$, recorded in $\mathrm{CD}_{3} \mathrm{CN}$ solution $\left(*=\mathrm{CD}_{3} \mathrm{CN} ;+=\mathrm{H}_{2} \mathrm{O}\right)$. 


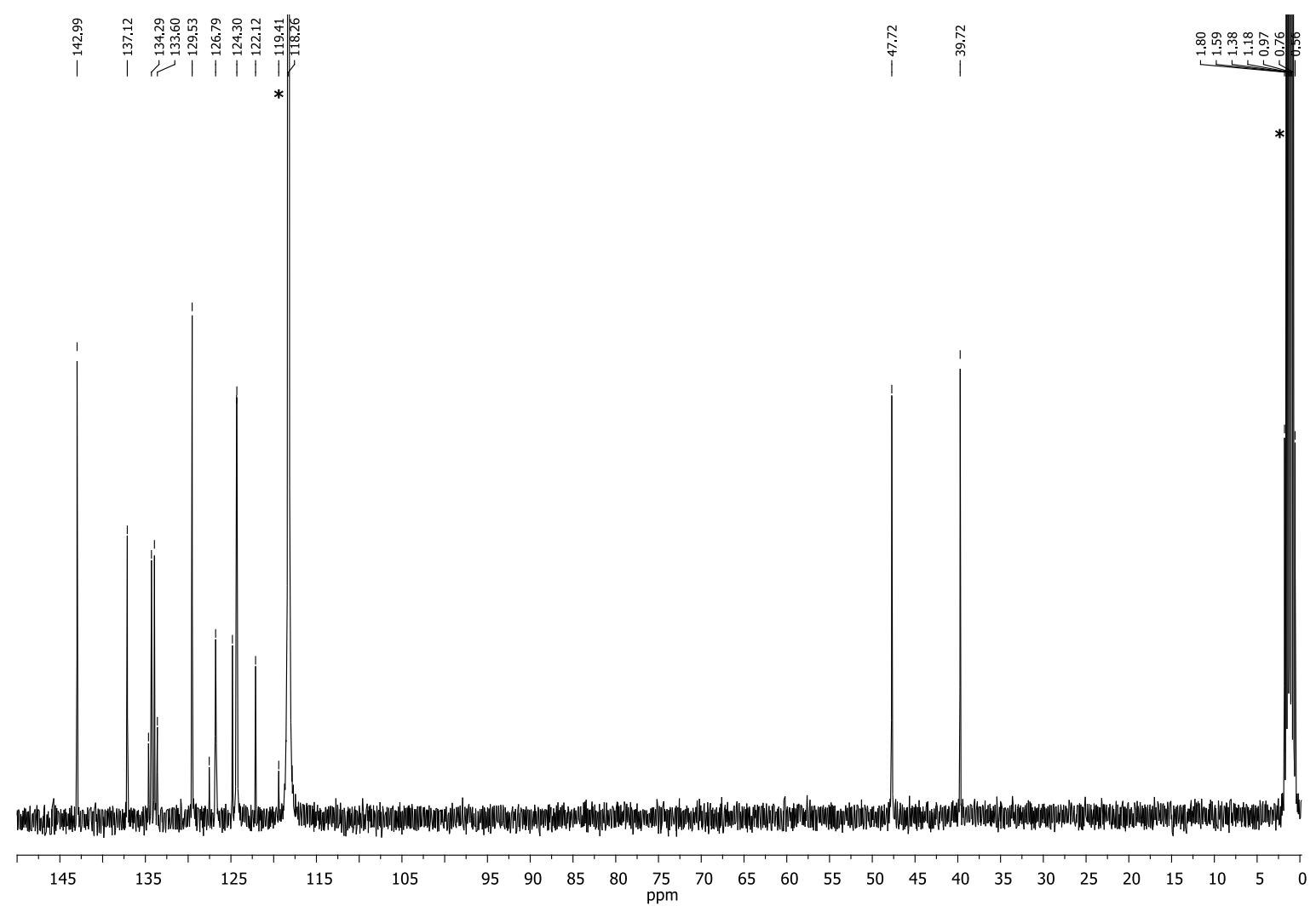

Figure S33. ${ }^{13} \mathrm{C}$ NMR spectrum of $[4 \mathrm{~d}]\left(\mathrm{BF}_{4}\right)_{3}$, recorded in $\mathrm{CD}_{3} \mathrm{CN}\left({ }^{*}\right)$ solution.

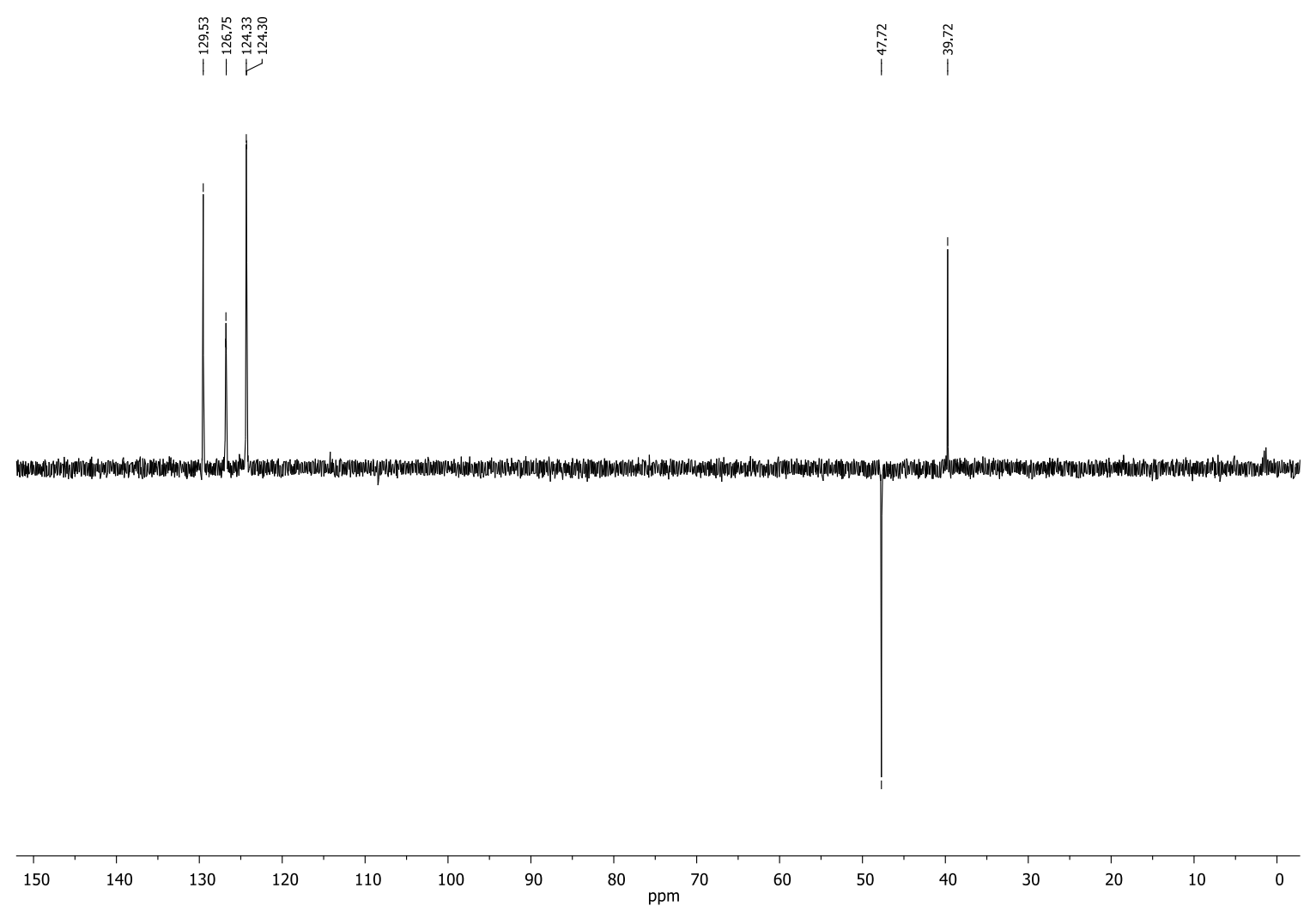

Figure S34. DEPT-135 NMR spectrum of $[4 \mathrm{~d}]\left(\mathrm{BF}_{4}\right)_{3}$, recorded in $\mathrm{CD}_{3} \mathrm{CN}$ solution. 


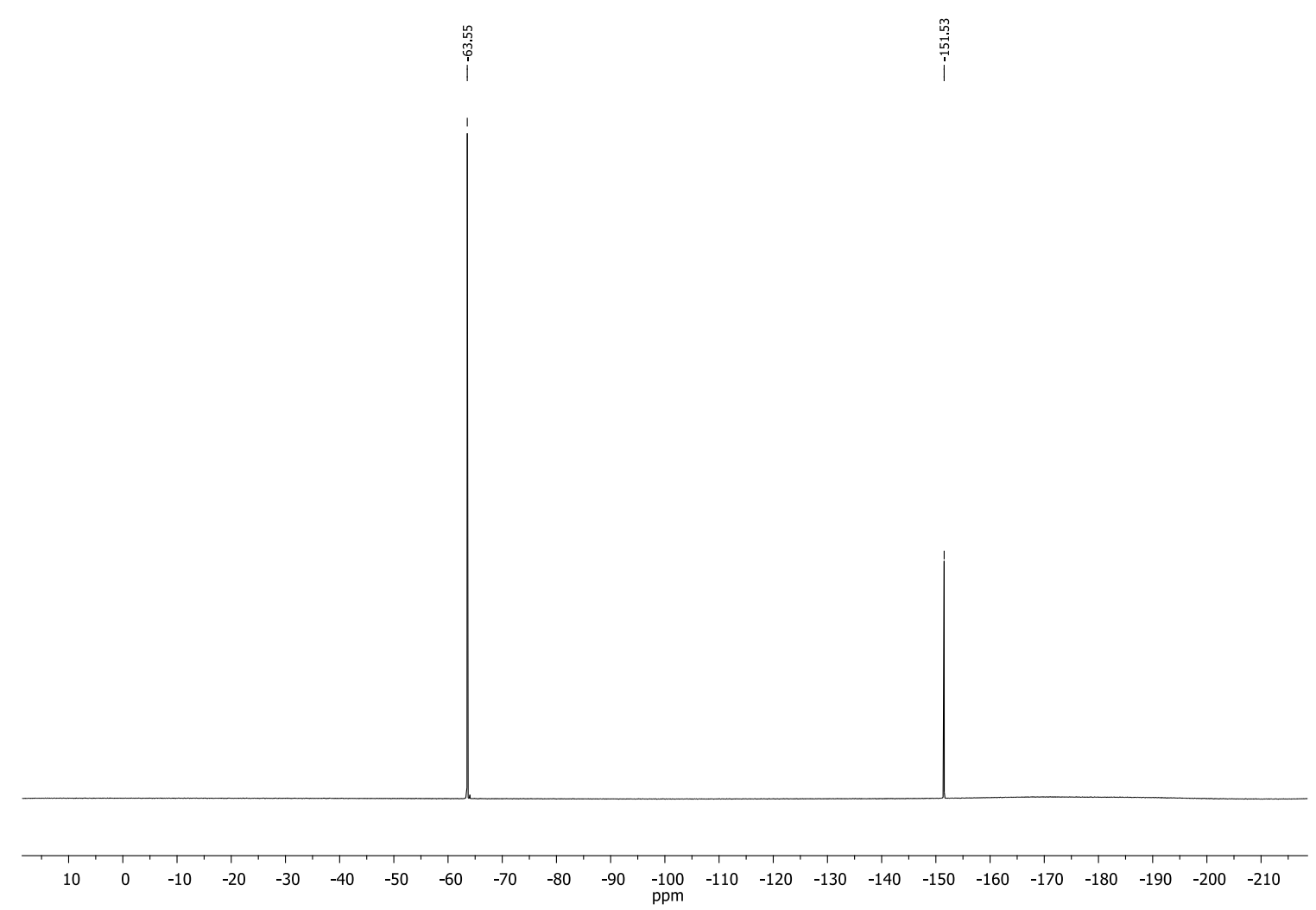

Figure S35. ${ }^{19} \mathrm{~F}$ NMR spectrum of $[4 \mathrm{~d}]\left(\mathrm{BF}_{4}\right)_{3}$, recorded in $\mathrm{CD}_{3} \mathrm{CN}$ solution.

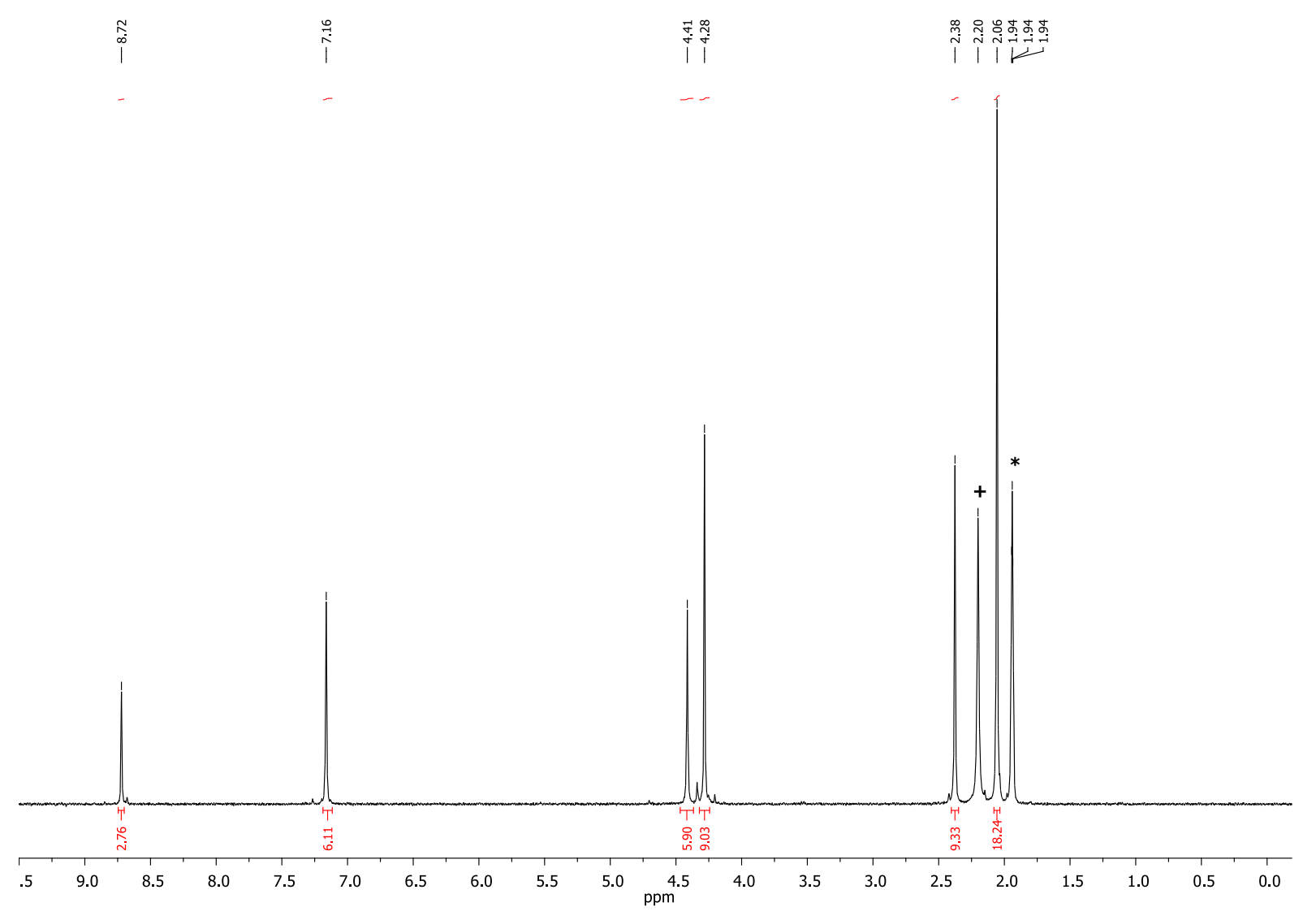

Figure S36. ${ }^{1} \mathrm{H}$ NMR spectrum of $[4 \mathrm{a}](\mathrm{OTf})_{3}$, recorded in $\mathrm{CD}_{3} \mathrm{CN}$ solution $\left(*=\mathrm{CD}_{3} \mathrm{CN} ;+=\mathrm{H}_{2} \mathrm{O}\right)$. 


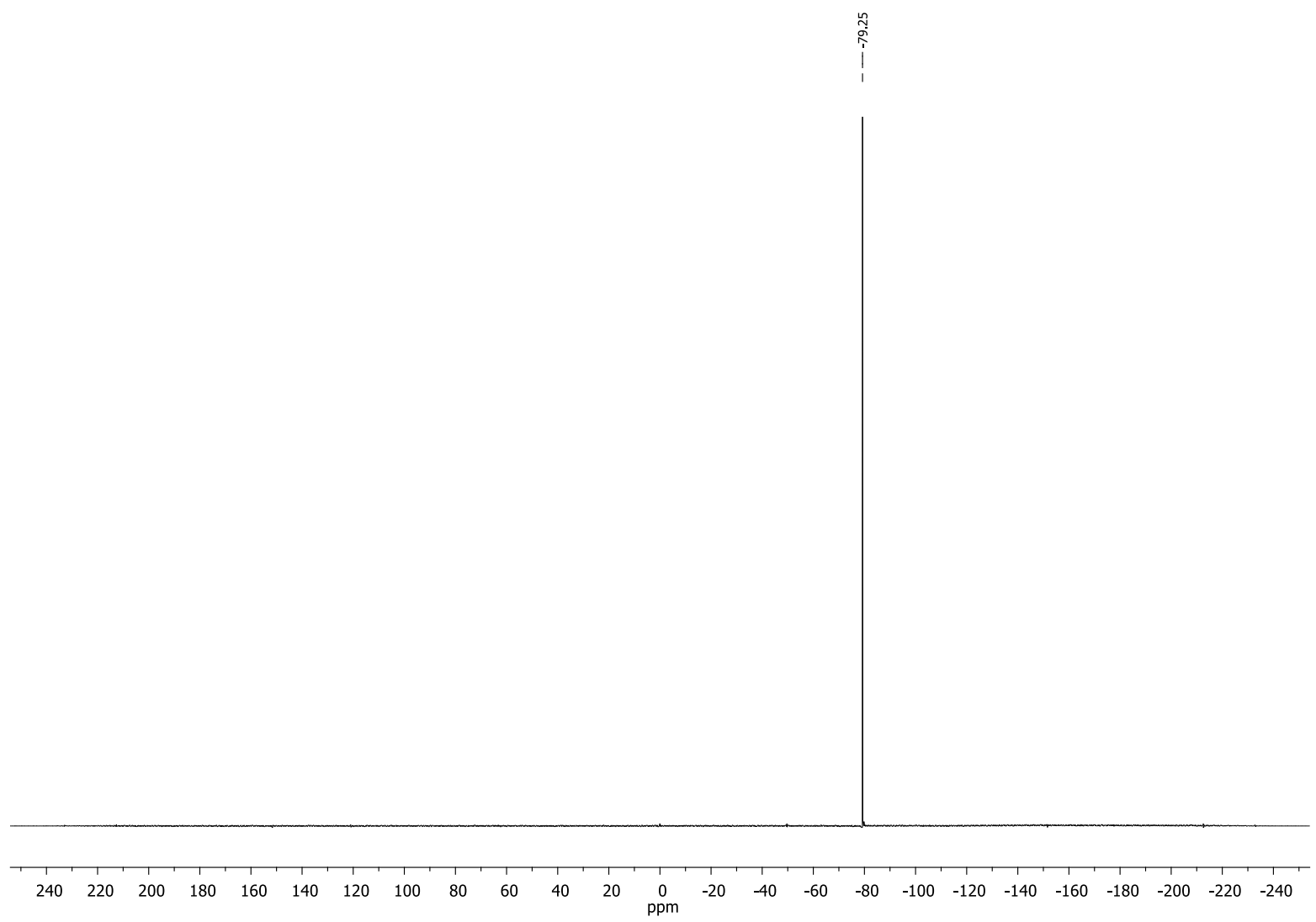

Figure S37. ${ }^{19} \mathrm{~F} N M R$ spectrum of $[4 \mathrm{a}](\mathrm{OTf})_{3}$, recorded in $\mathrm{CD}_{3} \mathrm{CN}$ solution.

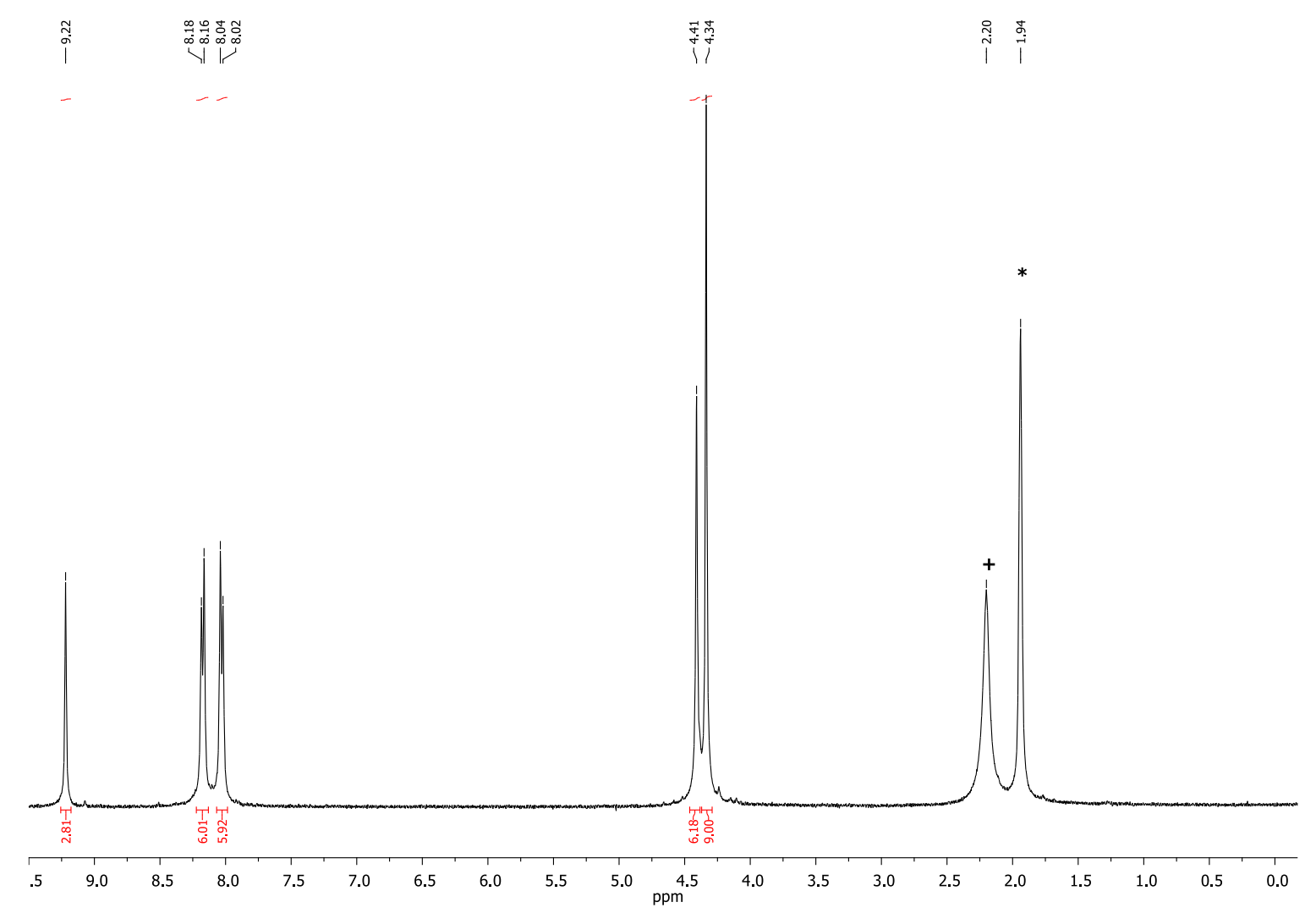

Figure S38. ${ }^{1} \mathrm{H}$ NMR spectrum of $[4 \mathbf{b}](\mathrm{OTf})_{3}$, recorded in $\mathrm{CD}_{3} \mathrm{CN}$ solution $\left(*=\mathrm{CD}_{3} \mathrm{CN} ;+=\mathrm{H}_{2} \mathrm{O}\right)$. 


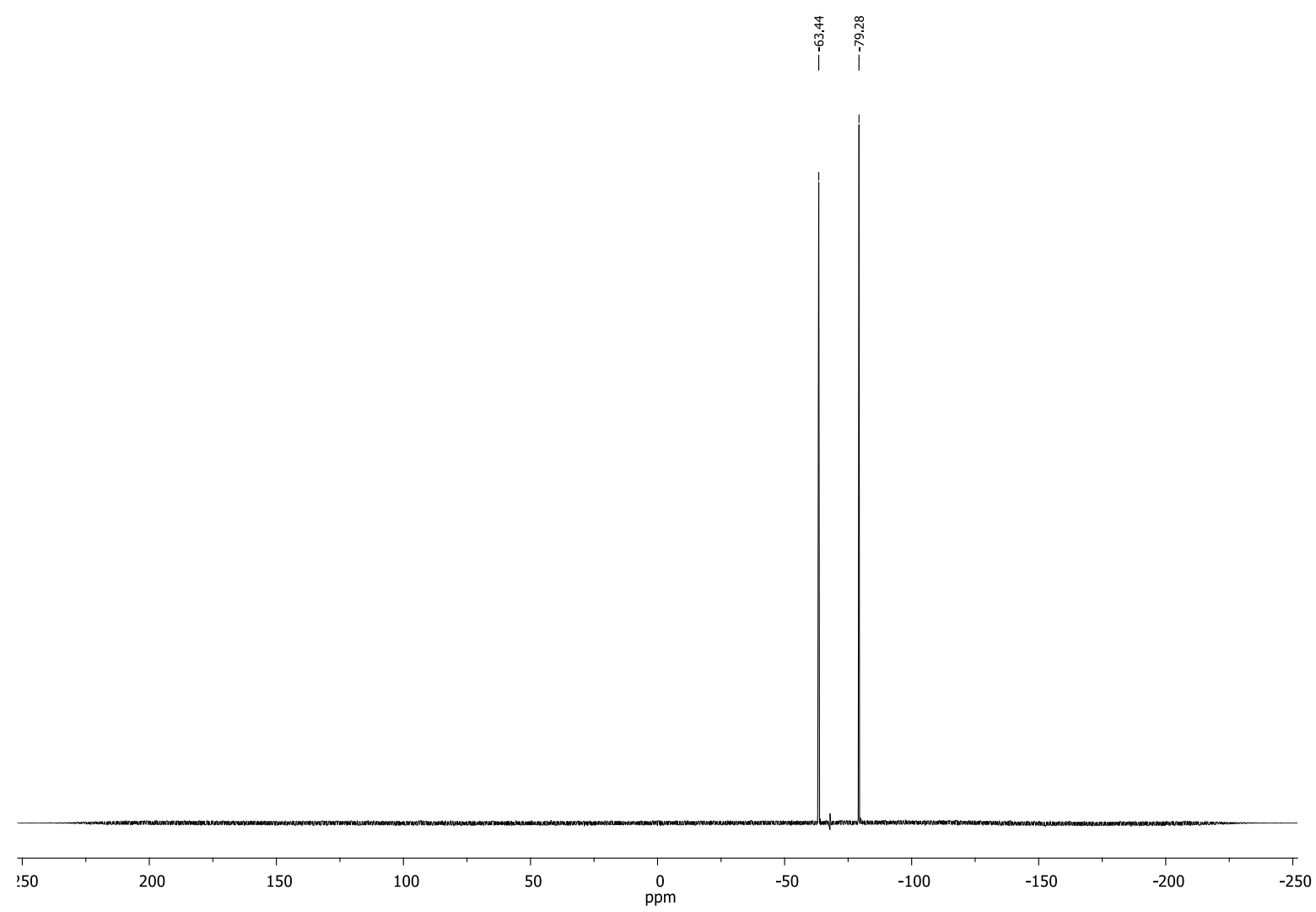

Figure S39. ${ }^{19} \mathrm{~F} N M R$ spectrum of $[4 \mathbf{b}](\mathrm{OTf})_{3}$, recorded in $\mathrm{CD}_{3} \mathrm{CN}$ solution.

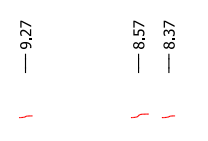

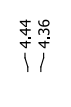

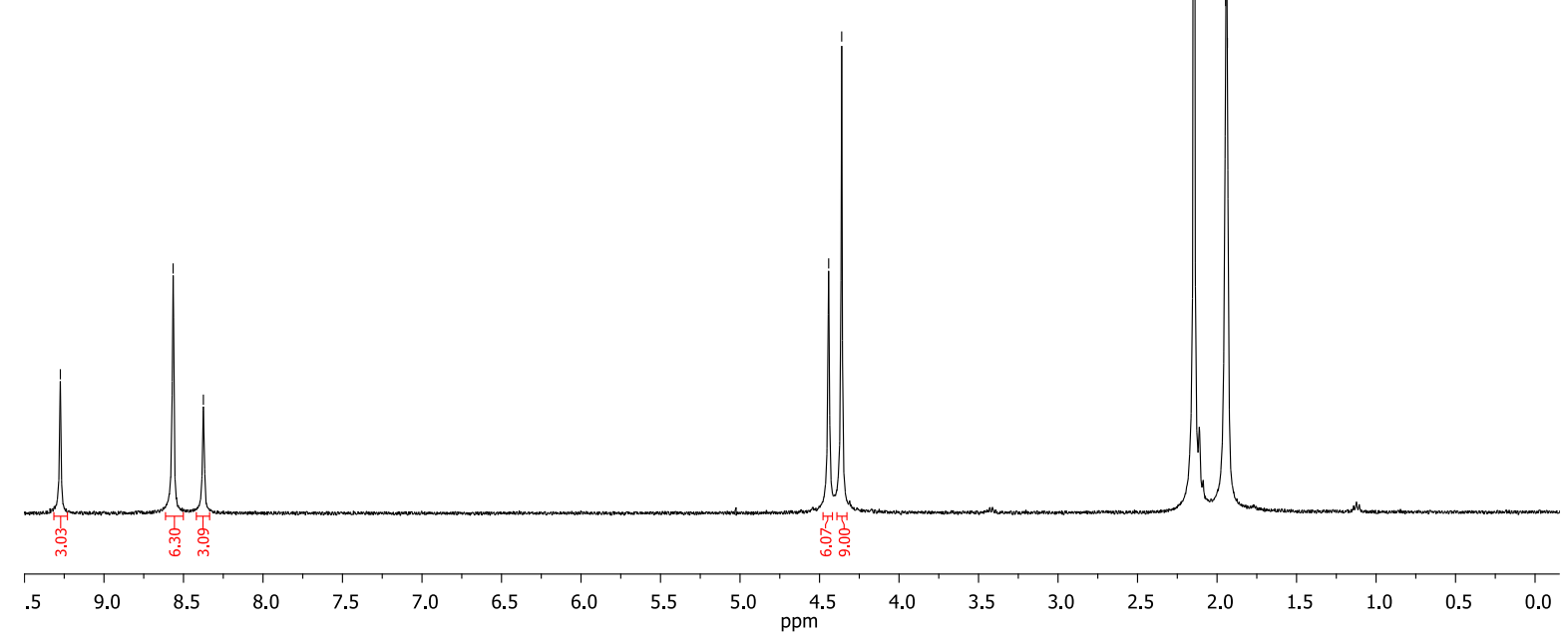

Figure S40. ${ }^{1} \mathrm{H}$ NMR spectrum of $[4 \mathrm{~d}](\mathrm{OTf})_{3}$, recorded in $\mathrm{CD}_{3} \mathrm{CN}$ solution $\left({ }^{*}=\mathrm{CD}_{3} \mathrm{CN} ;+=\mathrm{H}_{2} \mathrm{O}\right)$.

S33 


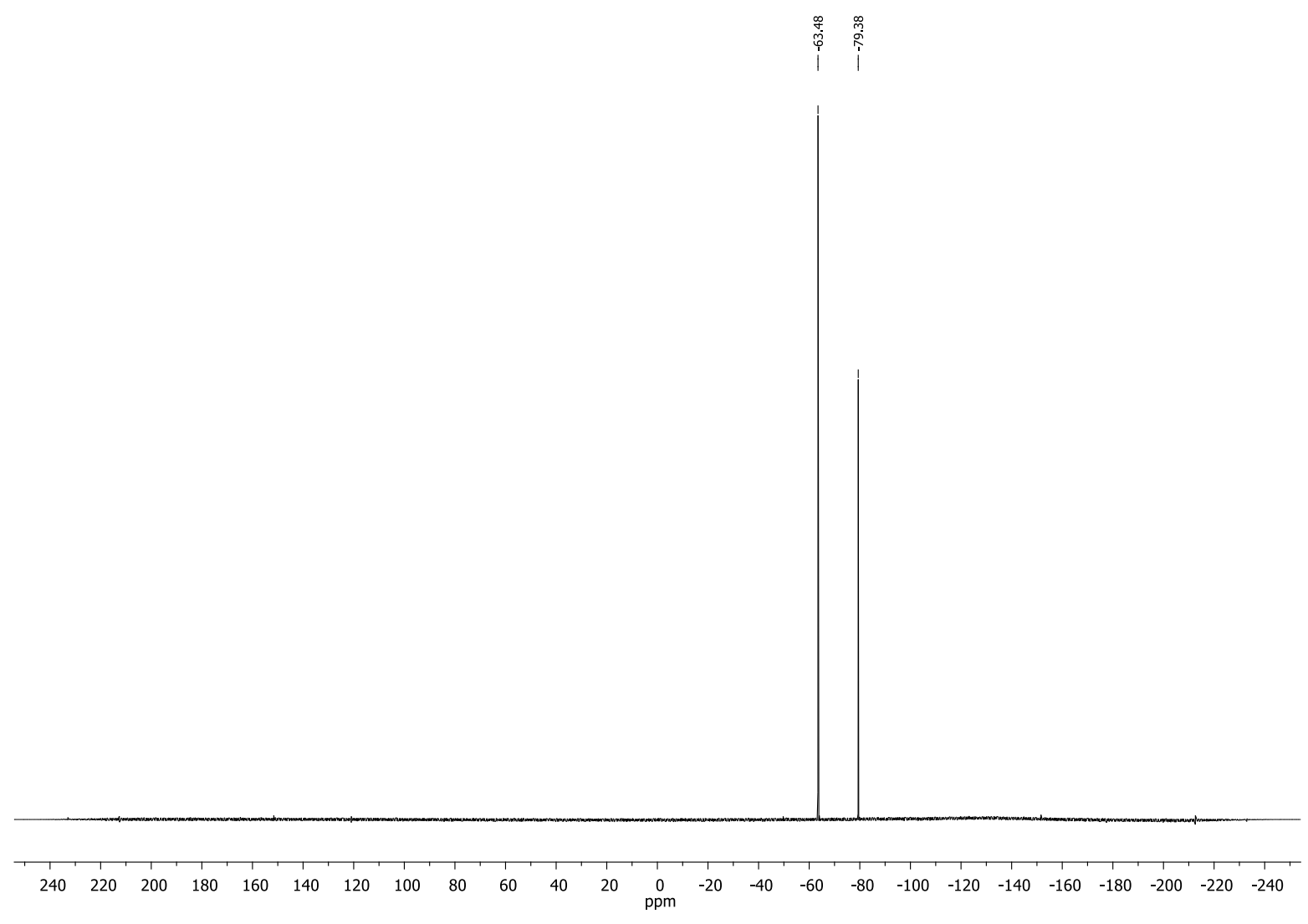

Figure $\mathrm{S41.}{ }^{19} \mathrm{~F}$ NMR spectrum of $[\mathbf{4 d}](\mathrm{OTf})_{3}$, recorded in $\mathrm{CD}_{3} \mathrm{CN}$ solution.

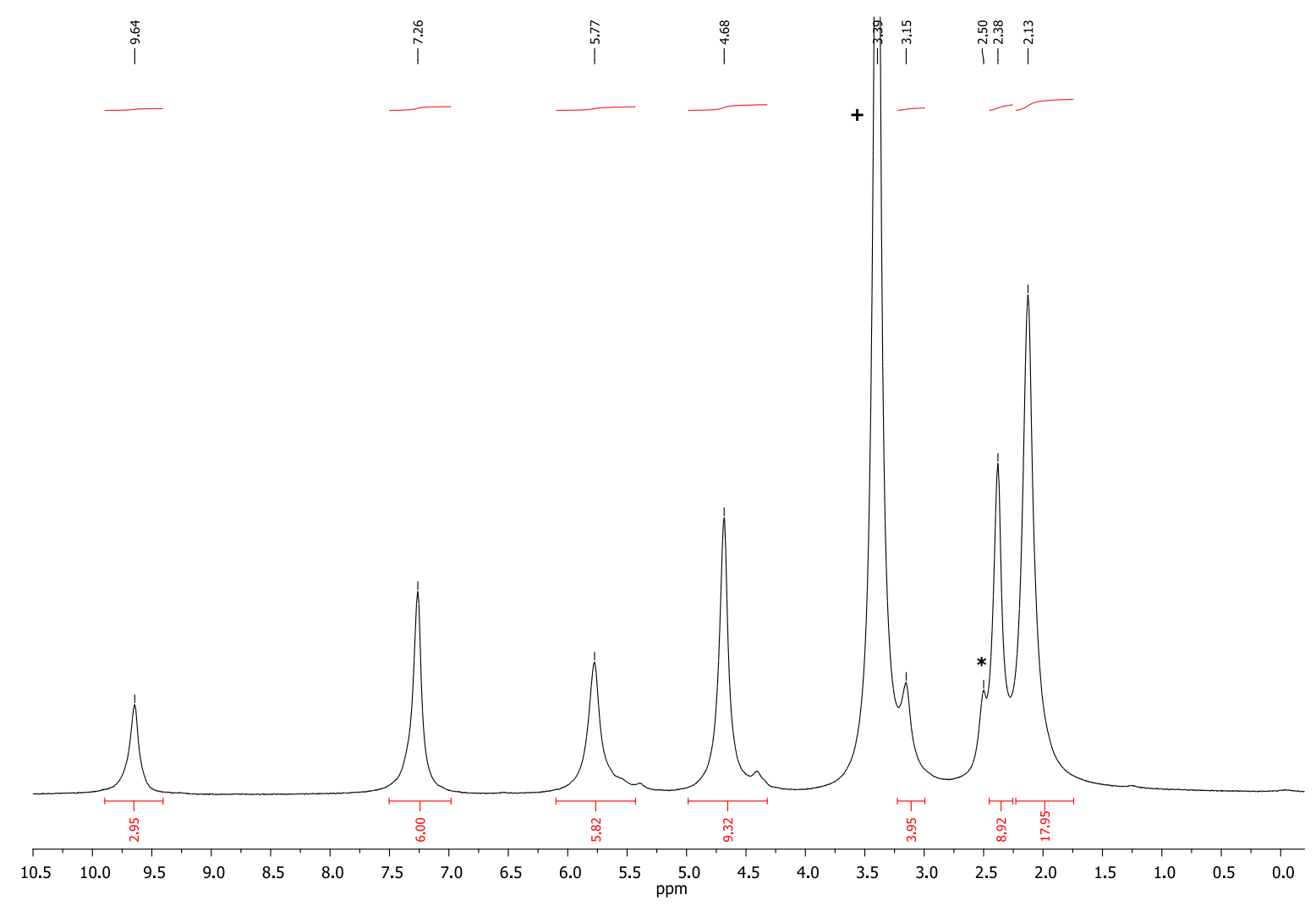

Figure S42. ${ }^{1} \mathrm{H}$ NMR spectrum of $[5 a]\left(\mathrm{BF}_{4}\right)_{4}$, recorded in DMSO- $\mathrm{d}_{6}$ solution $\left(*=\mathrm{DMSO}-\mathrm{d}_{6} ;+=\mathrm{H}_{2} \mathrm{O}\right)$. 


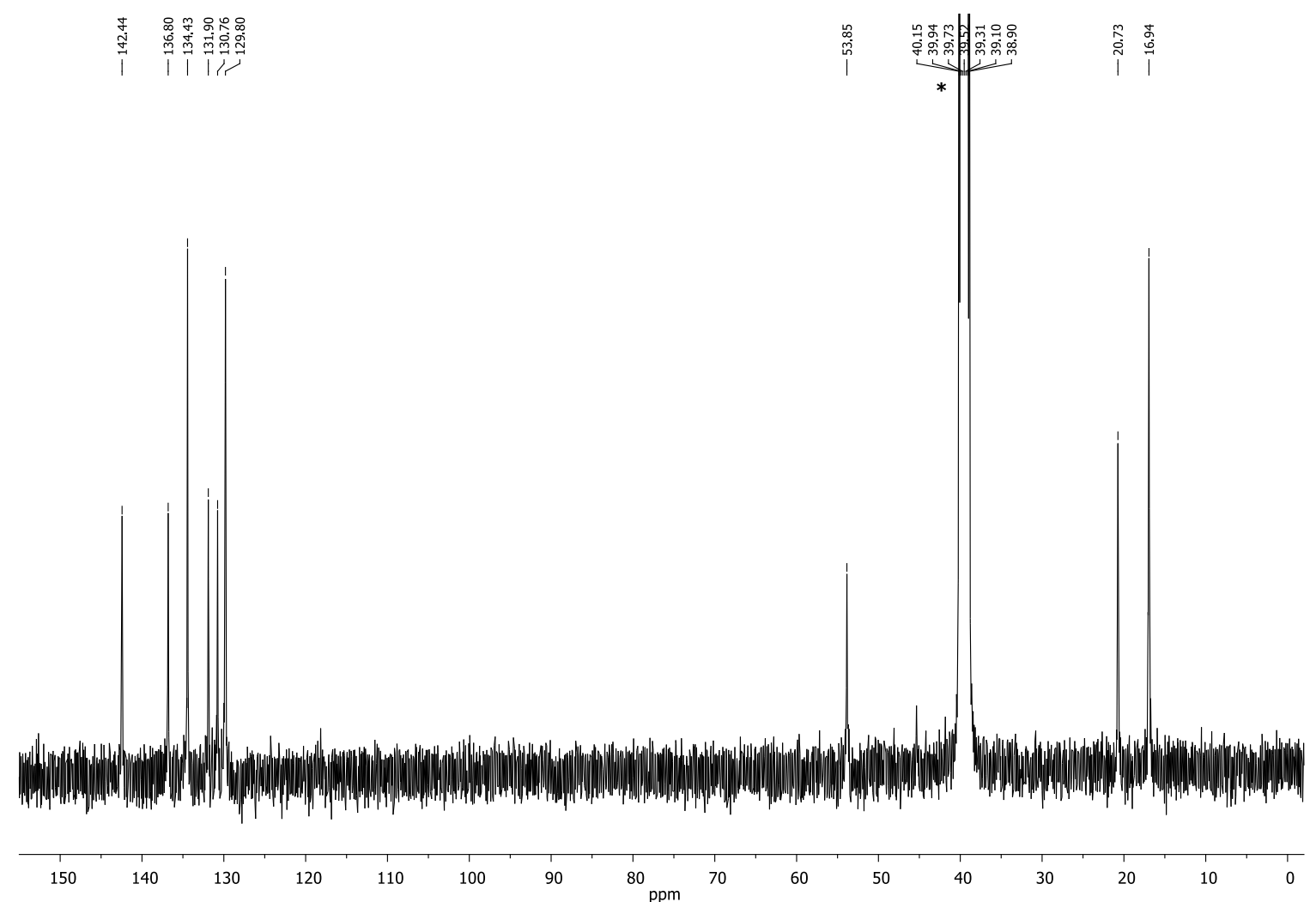

Figure S43. ${ }^{13} \mathrm{C}$ NMR spectrum of $[5 a]\left(\mathrm{BF}_{4}\right)_{4}$, recorded in DMSO-d ${ }_{6}\left({ }^{*}\right)$ solution.DMSO- $\mathrm{d}_{6}$.

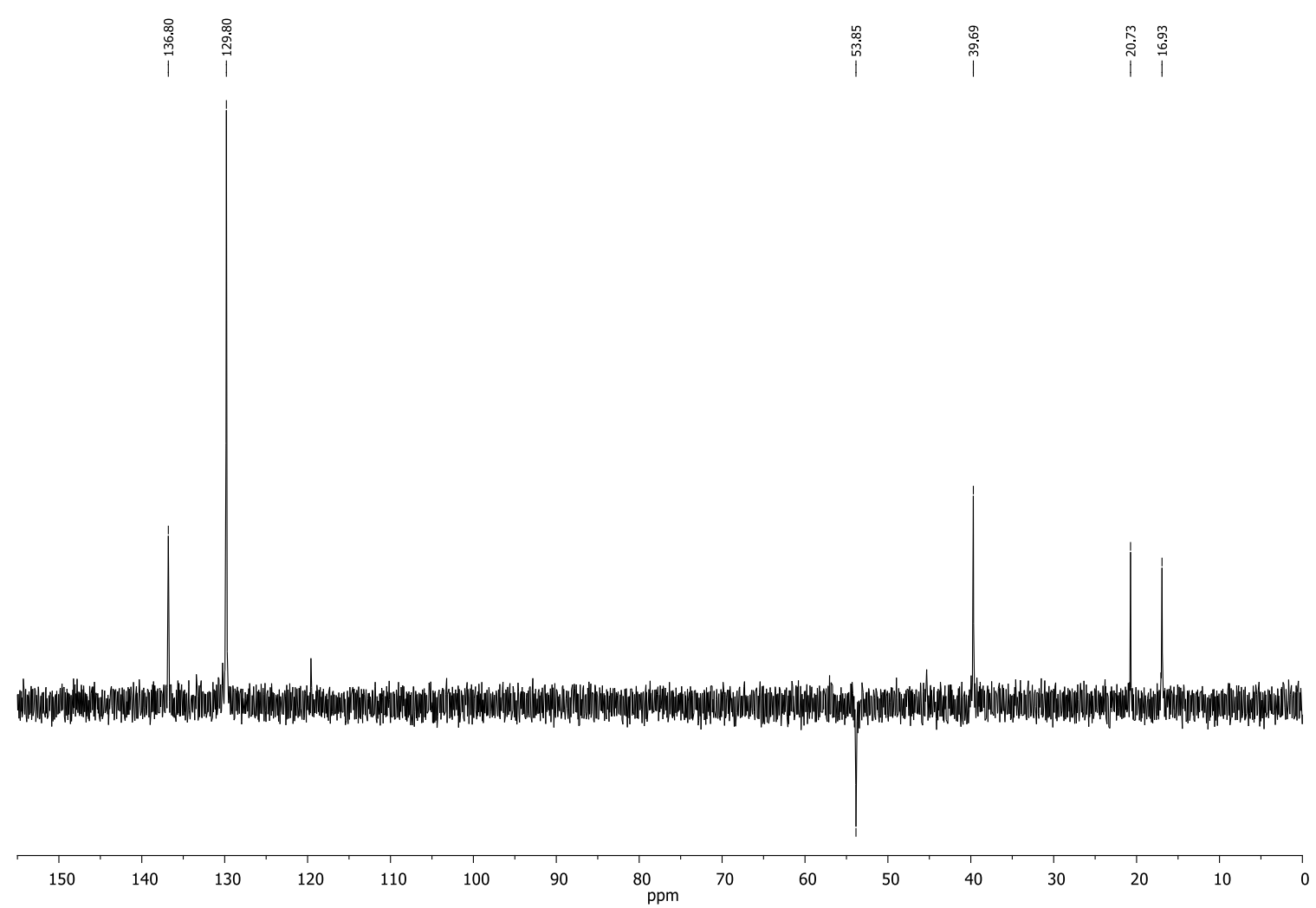

Figure S44. DEPT-135 NMR spectrum of $[5 a]\left(\mathrm{BF}_{4}\right)_{4}$, recorded in DMSO-d 6 solution. 


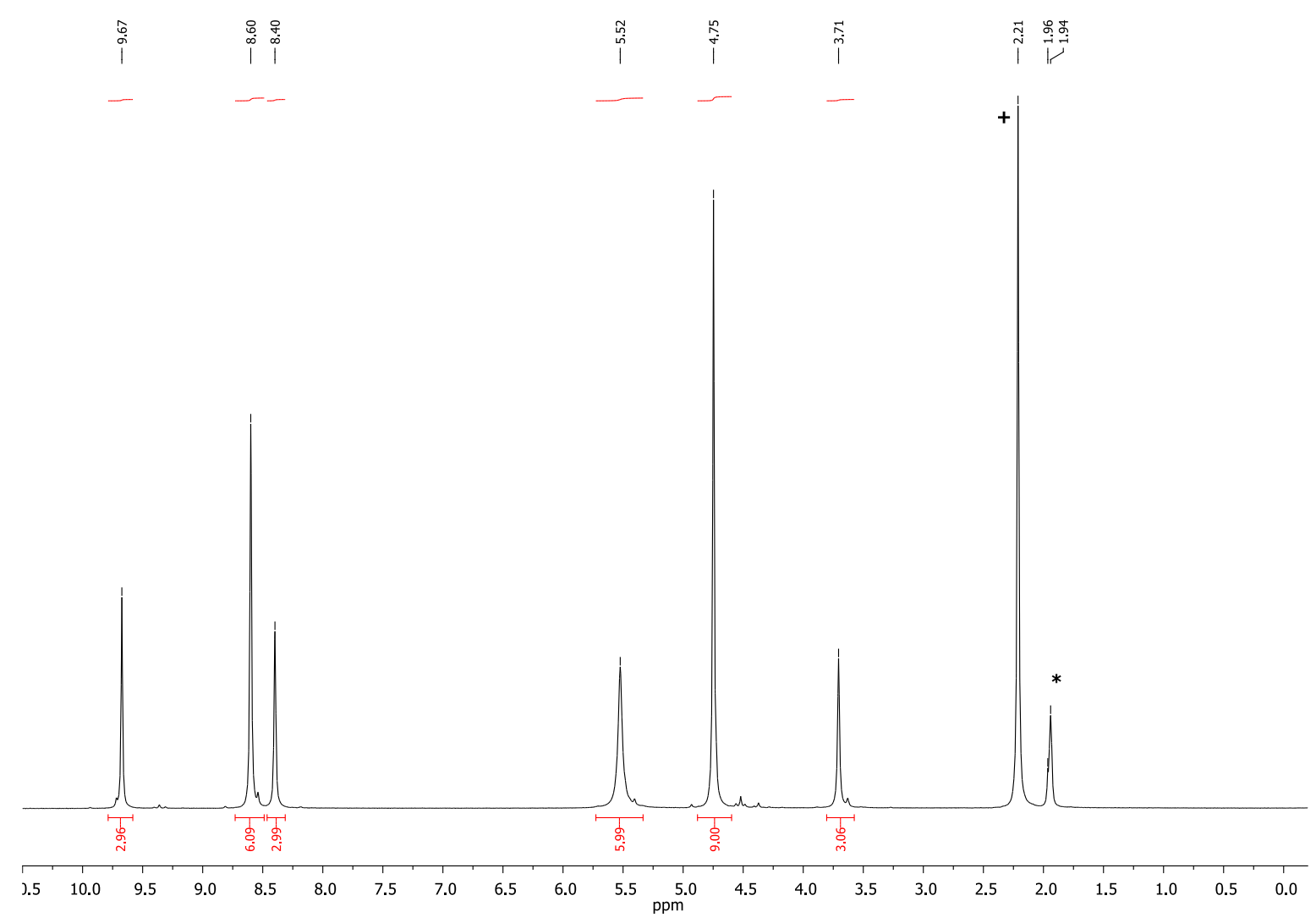

Figure S45. ${ }^{1} \mathrm{H}$ NMR spectrum of $[5 \mathrm{~d}](\mathrm{OTf})_{4}$, recorded in $\mathrm{CD}_{3} \mathrm{CN}$ solution $\left(*=\mathrm{CD}_{3} \mathrm{CN} ;+=\mathrm{H}_{2} \mathrm{O}\right)$.

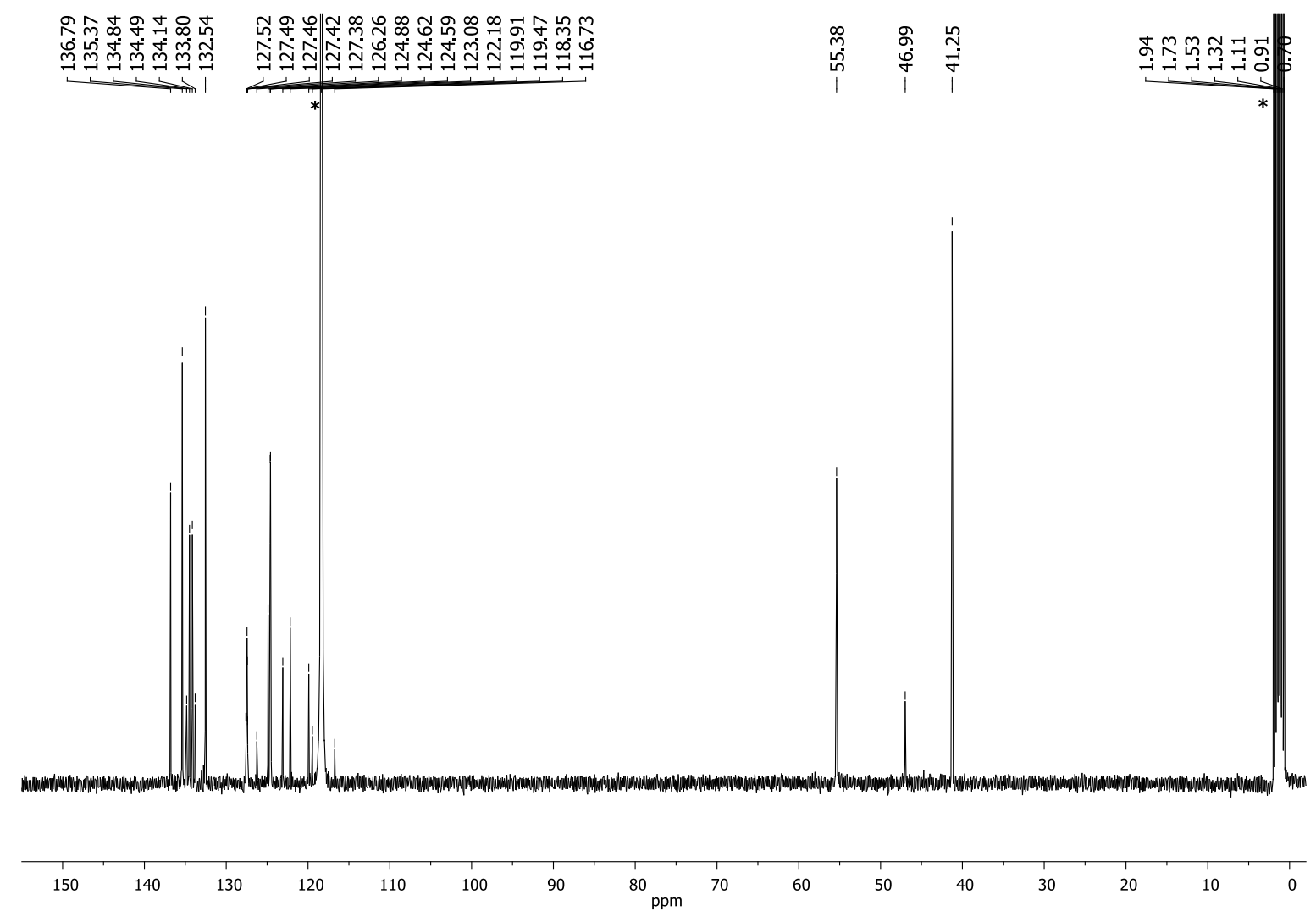

Figure S46. ${ }^{13} \mathrm{C}$ NMR spectrum of $[\mathbf{5 d}](\mathrm{OTf})_{4}$, recorded in $\mathrm{CD}_{3} \mathrm{CN}\left({ }^{*}\right)$ solution. 


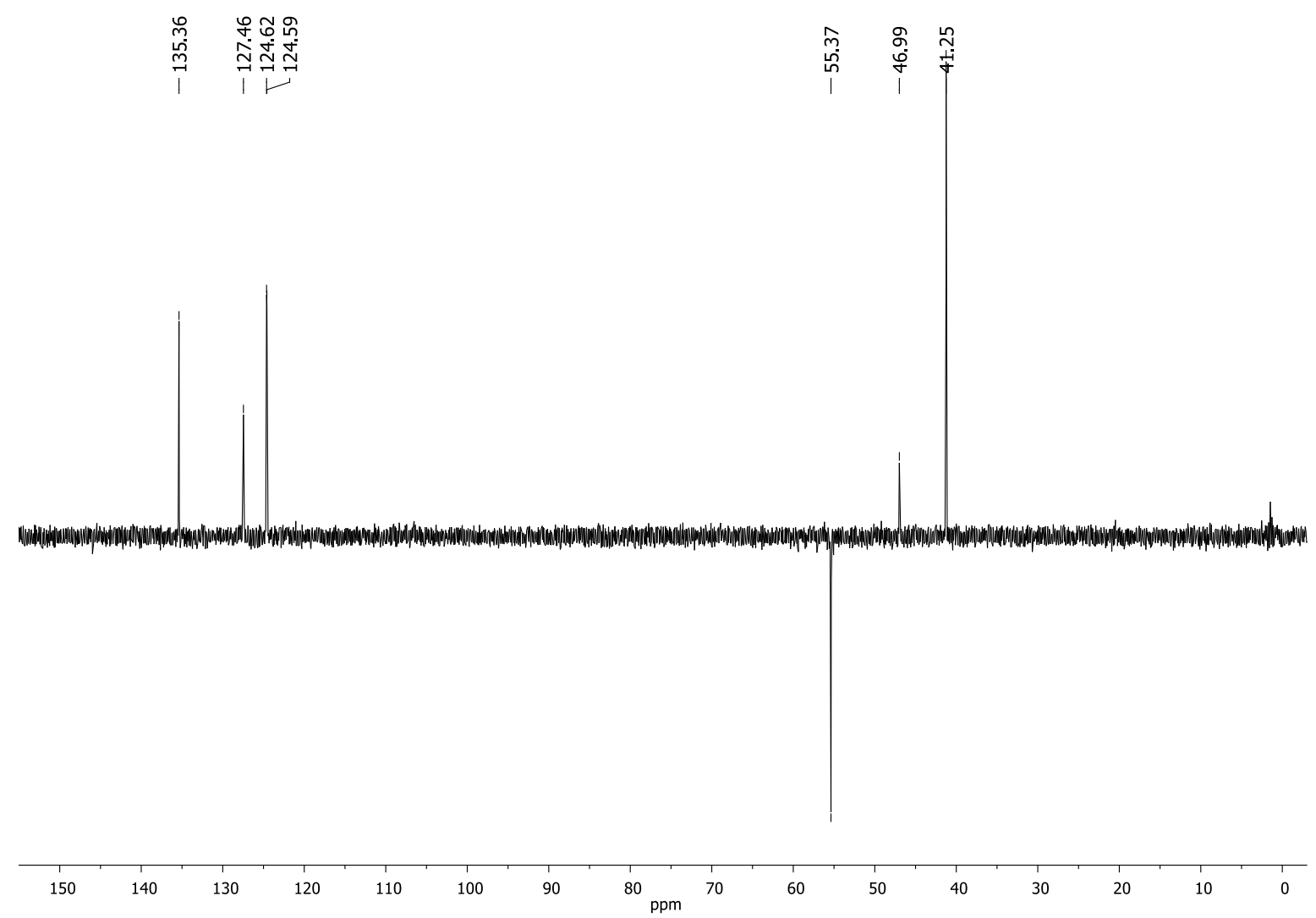

Figure S47. DEPT-135 spectrum of [5d](OTf $)_{4}$, recorded in $\mathrm{CD}_{3} \mathrm{CN}$ solution.

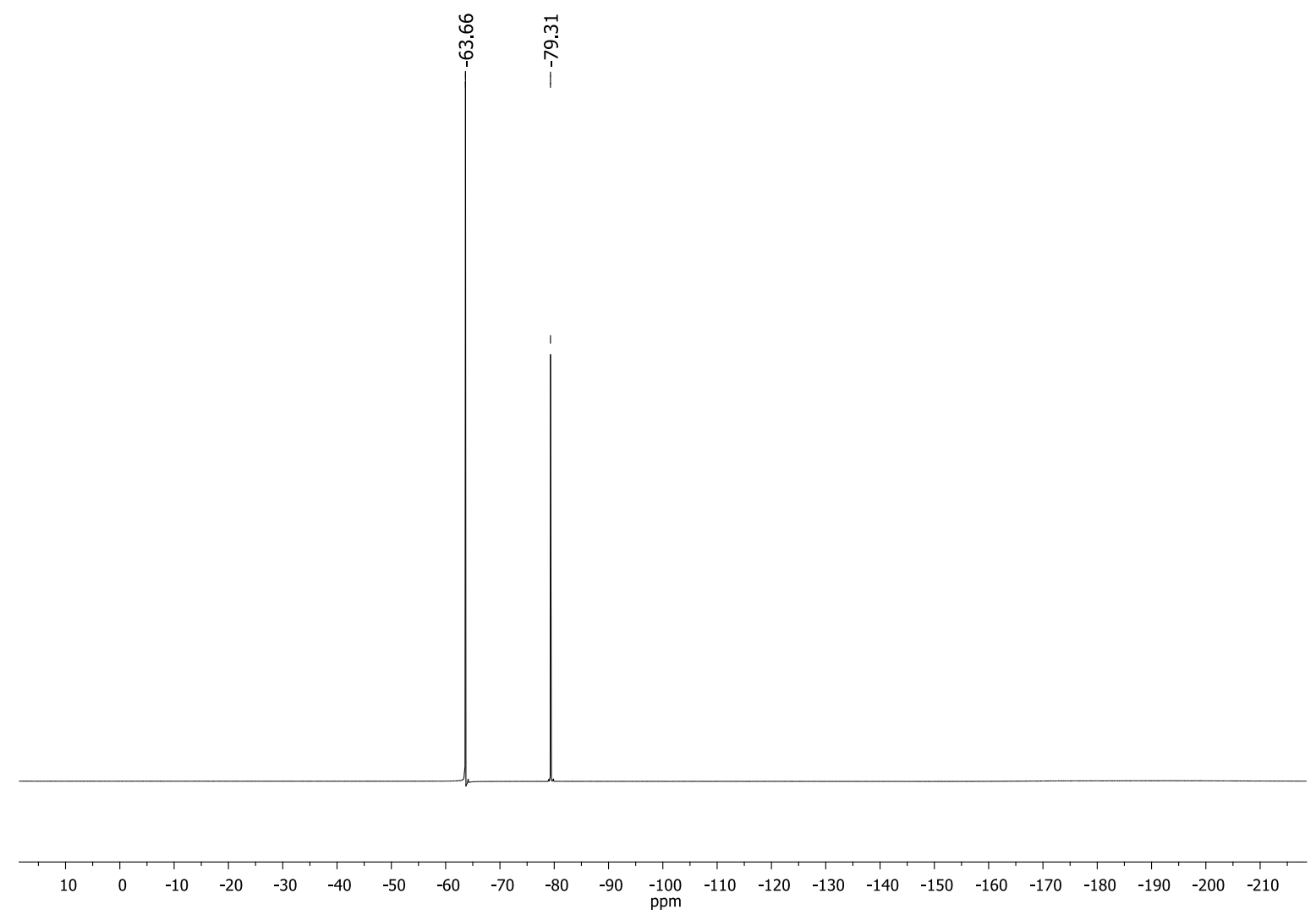

Figure S48. ${ }^{19} \mathrm{~F}$ NMR spectrum of $[5 \mathrm{~d}](\mathrm{OTf})_{4}$, recorded in $\mathrm{CD}_{3} \mathrm{CN}$ solution. 


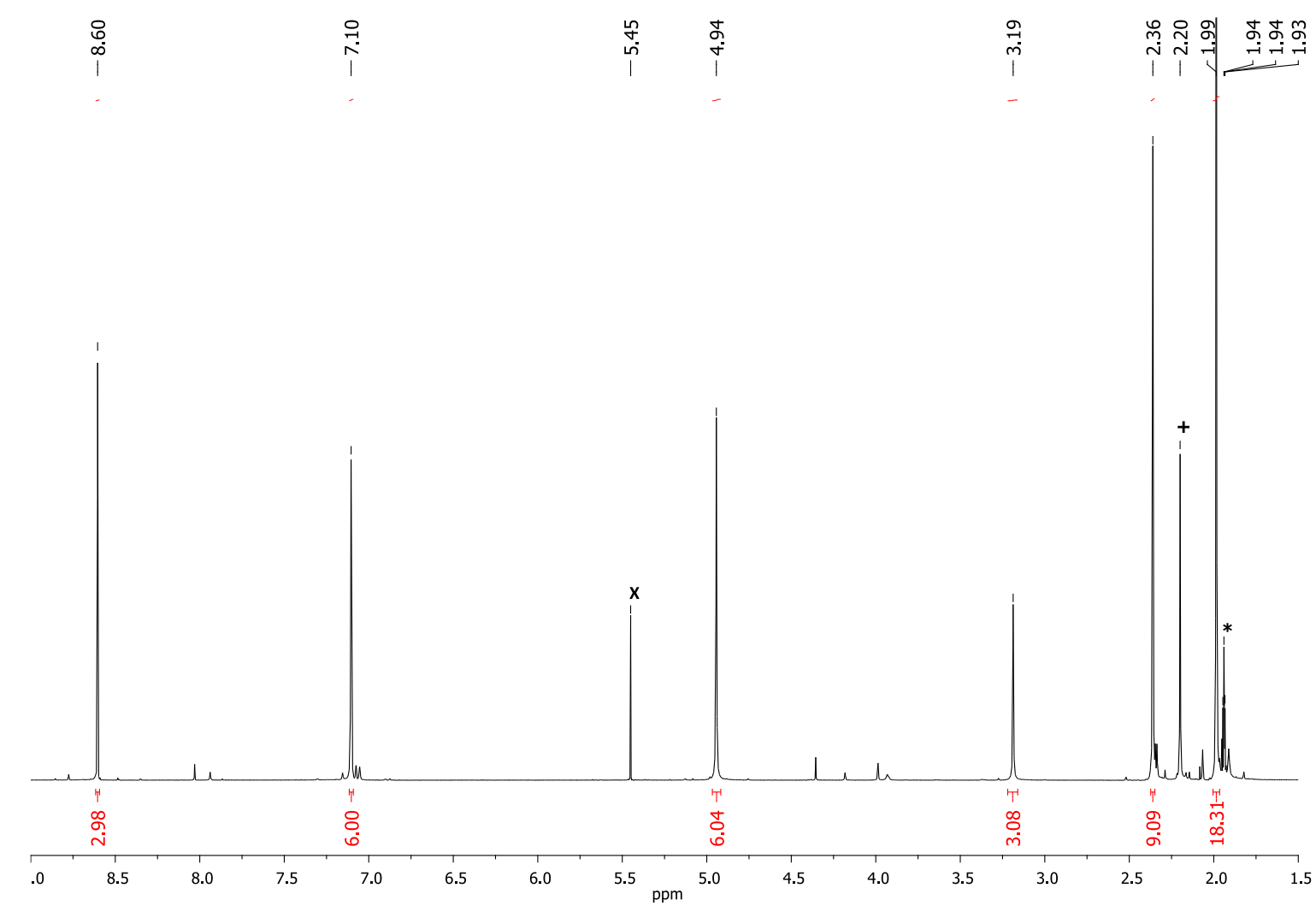

Figure S49. ${ }^{1} \mathrm{H}$ NMR spectrum of $[6 a](I)$, recorded in $\mathrm{CD}_{3} \mathrm{CN}$ solution $\left(*=\mathrm{CD}_{3} \mathrm{CN} ;+=\mathrm{H}_{2} \mathrm{O} ; \mathbf{x}=\mathrm{CH}_{2} \mathrm{Cl}_{2}\right)$.

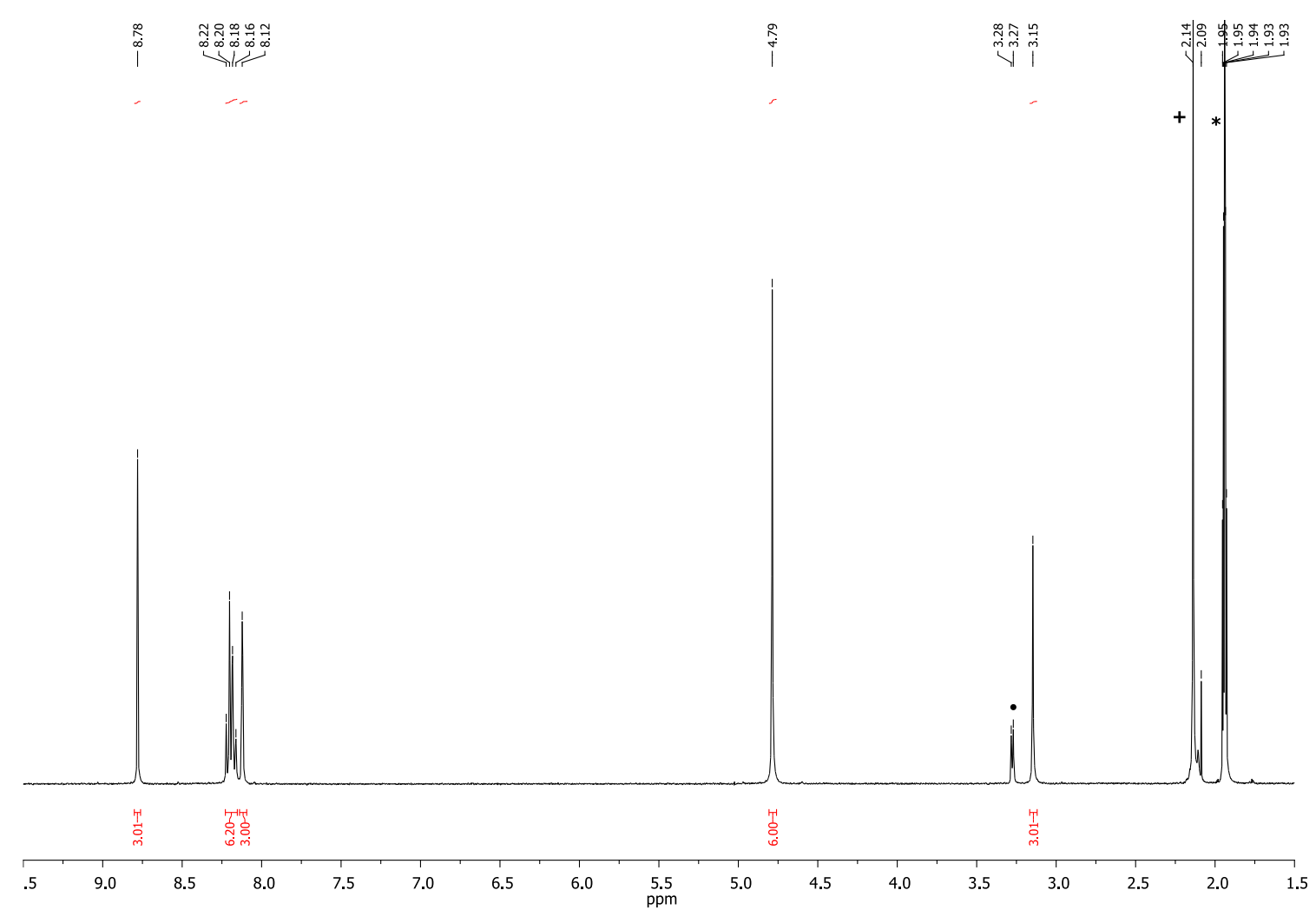

Figure S50. ${ }^{1} \mathrm{H}$ NMR spectrum of $[6 \mathrm{c}](\mathrm{OTf})$, recorded in $\mathrm{CD}_{3} \mathrm{CN}$ solution $\left({ }^{*}=\mathrm{CD}_{3} \mathrm{CN} ;+=\mathrm{H}_{2} \mathrm{O} ; \mathbf{x}=\mathrm{MeOH}\right)$. 


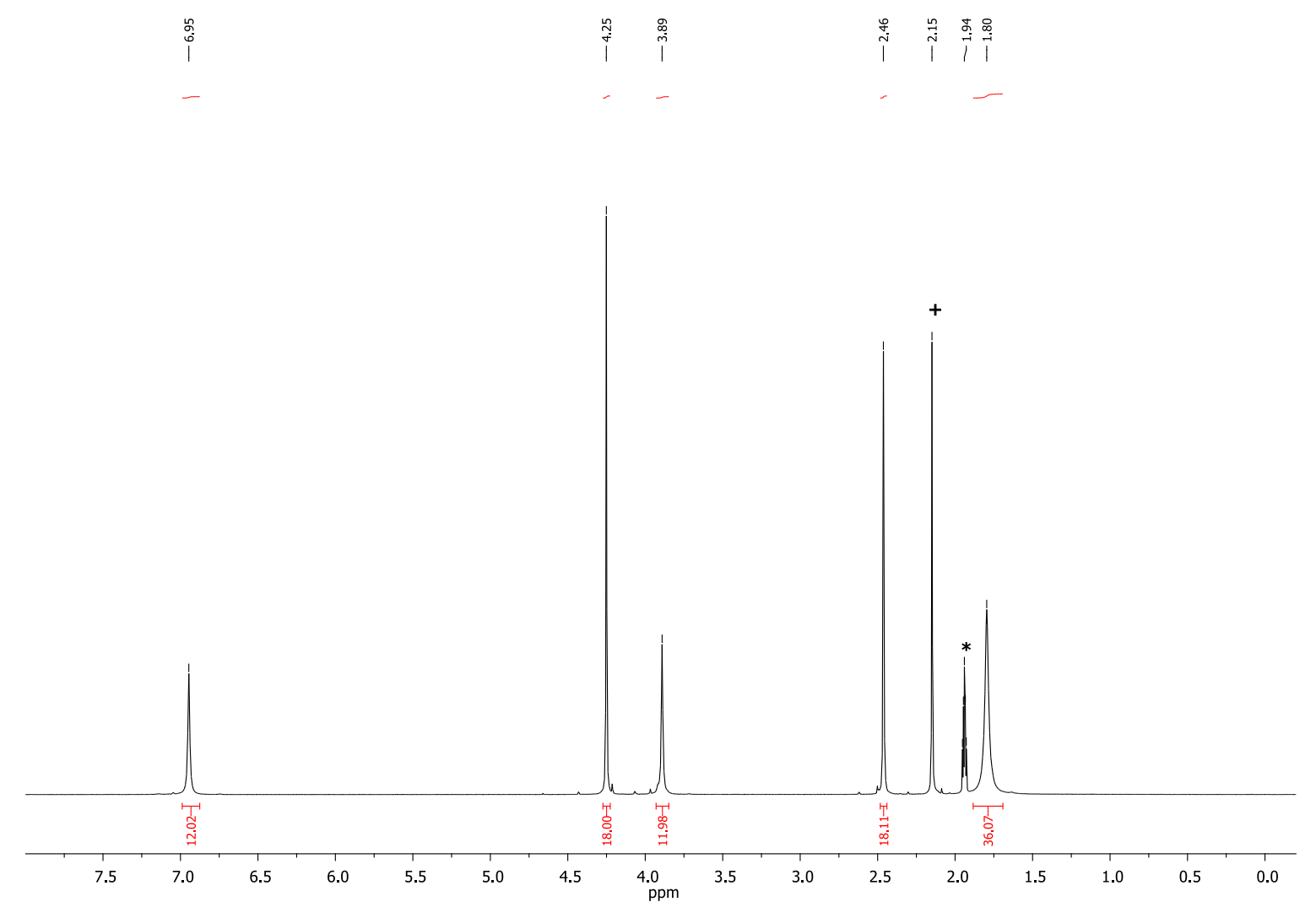

Figure S51. ${ }^{1} \mathrm{H}$ NMR spectrum of $\left[\mathrm{Ag}_{3}(\mathbf{1 a})_{2}\right]\left(\mathrm{BF}_{4}\right)_{3}$, recorded in $\mathrm{CD}_{3} \mathrm{CN}$ solution $\left(*=\mathrm{CD}_{3} \mathrm{CN} ;+=\mathrm{H}_{2} \mathrm{O}\right)$.

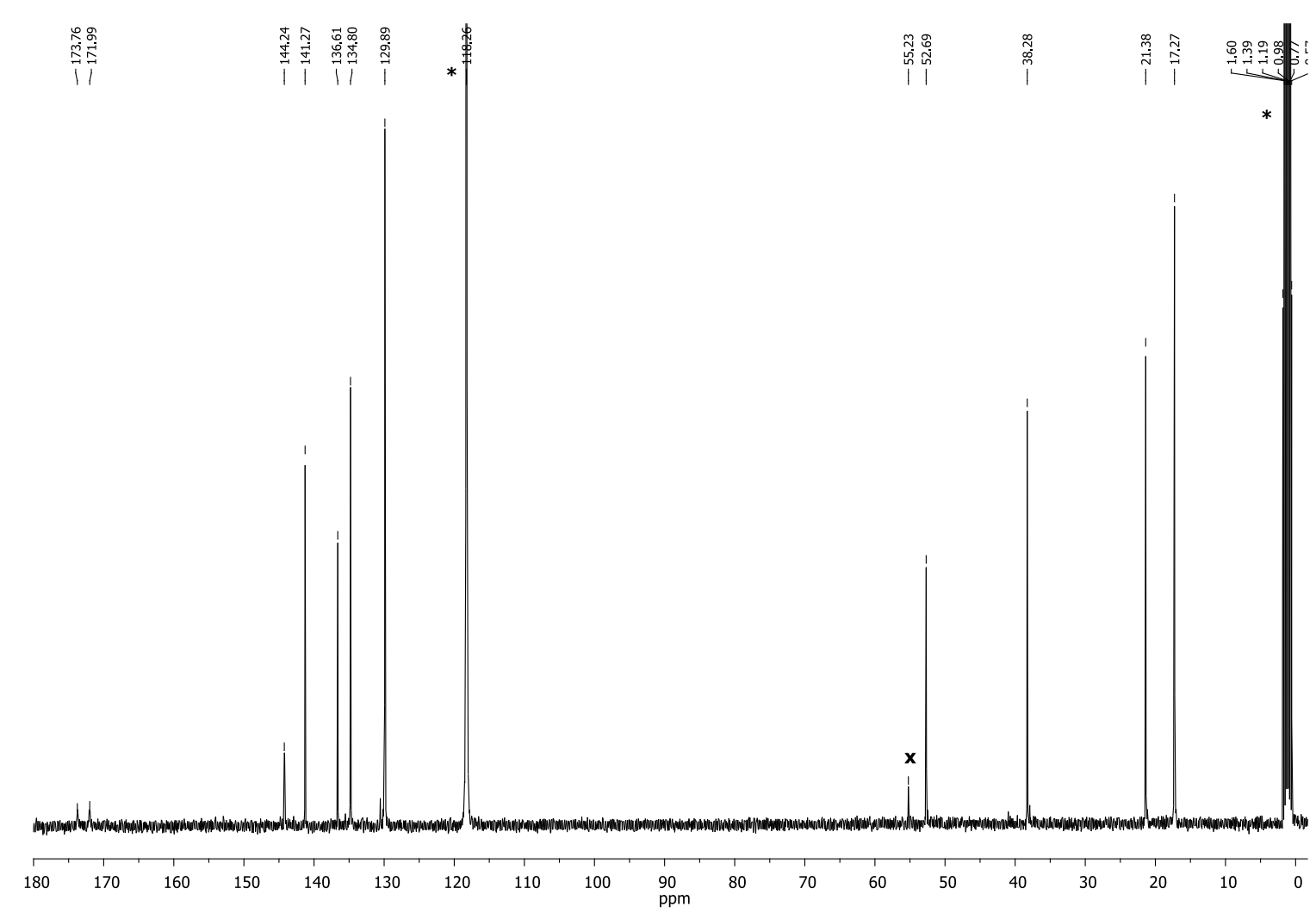

Figure S52. ${ }^{13} \mathrm{C}$ NMR spectrum of $\left[\mathrm{Ag}_{3}(\mathbf{1 a})_{2}\right]\left(\mathrm{BF}_{4}\right)_{3}$, recorded in $\mathrm{CD}_{3} \mathrm{CN}$ solution $\left(*=\mathrm{CD}_{3} \mathrm{CN} ; \mathbf{x}=\mathrm{CH}_{2} \mathrm{Cl}_{2}\right)$. 


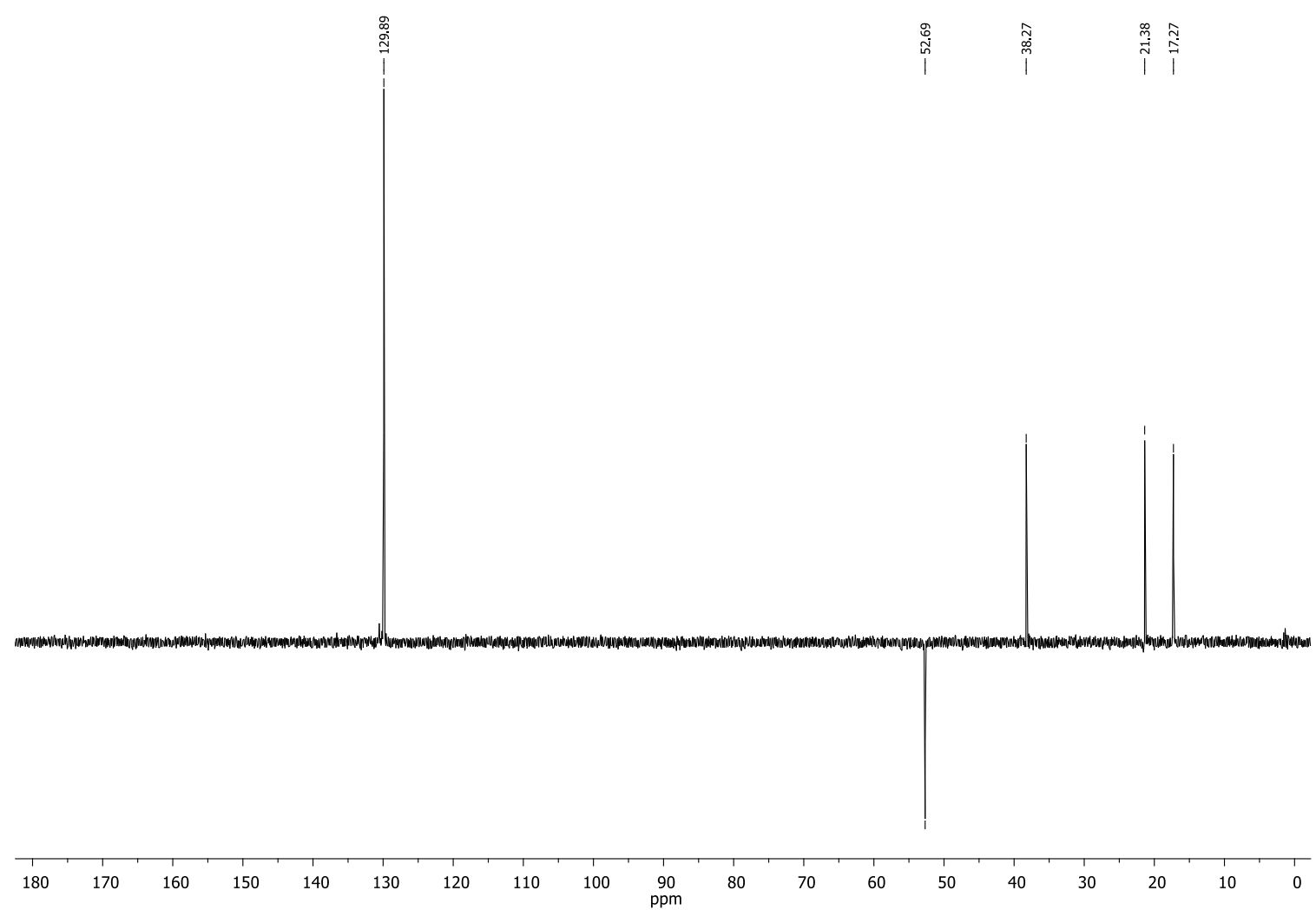

Figure S53. DEPT-135 NMR spectrum of $\left[\mathrm{Ag}_{3}(1 \mathrm{a})_{2}\right]\left(\mathrm{BF}_{4}\right)_{3}$, recorded in $\mathrm{CD}_{3} \mathrm{CN}$ solution.

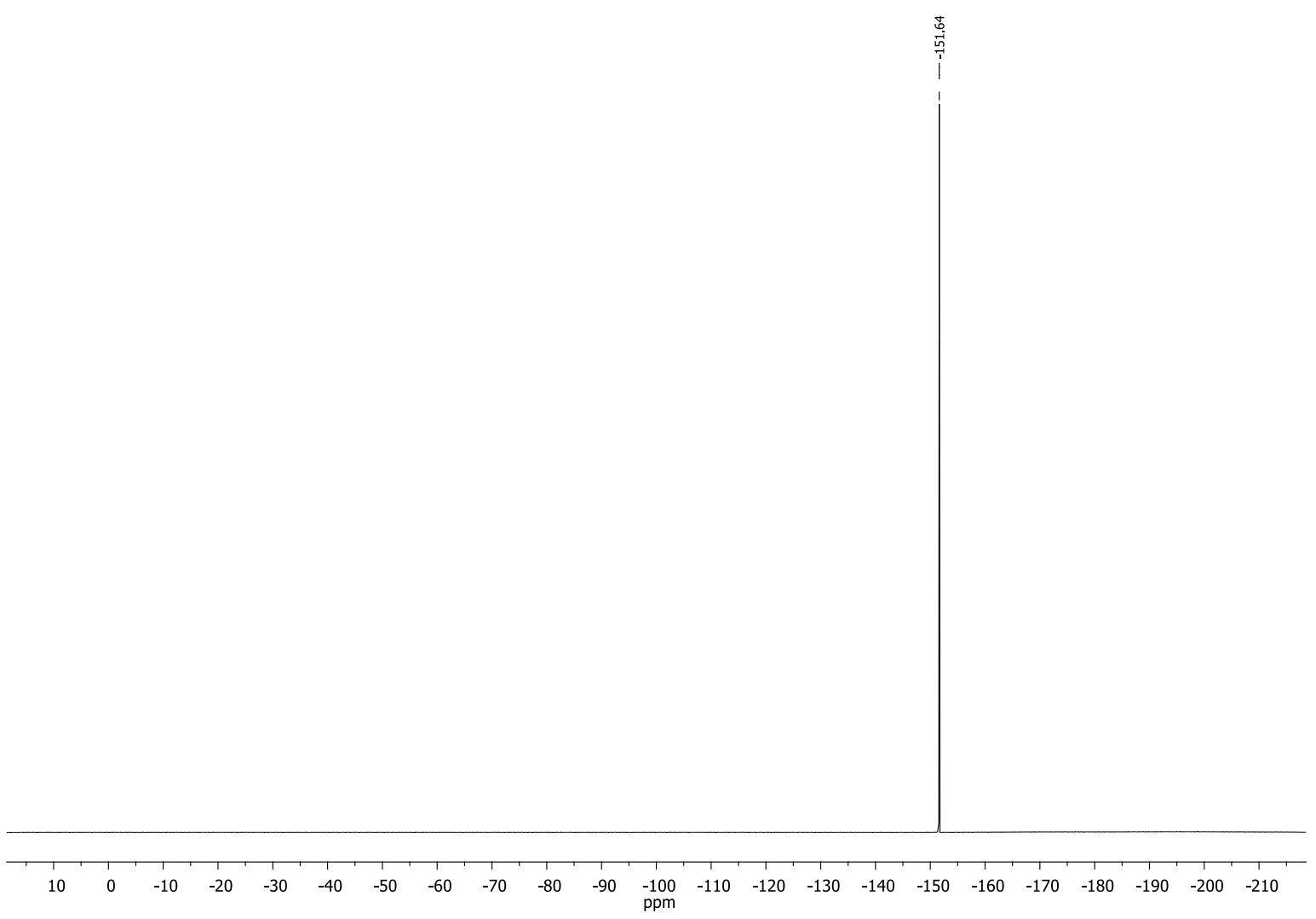

Figure $554 .{ }^{19} \mathrm{~F} N \mathrm{NR}$ spectrum of $\left[\mathrm{Ag}_{3}(1 \mathrm{a})_{2}\right]\left(\mathrm{BF}_{4}\right)_{3}$, recorded in $\mathrm{CD}_{3} \mathrm{CN}$ solution. 


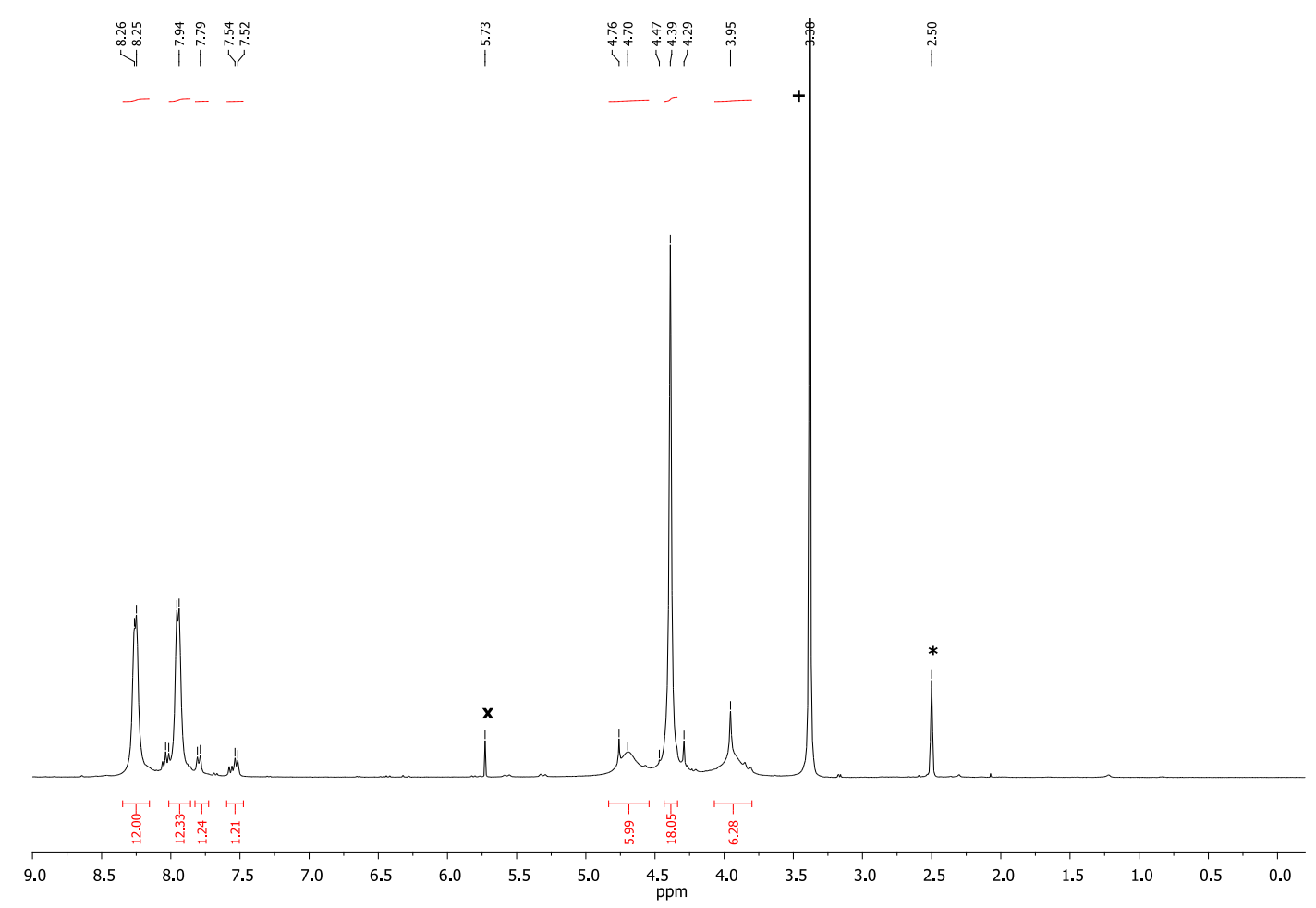

Figure S55. ${ }^{1} \mathrm{H}$ NMR spectrum of $\left[\mathrm{Ag}_{3}(\mathbf{1 b})_{2}\right]\left(\mathrm{BF}_{4}\right)_{3}$, recorded in DMSO- $\mathrm{d}_{6}$ solution $\left({ }^{*}=\mathrm{DMSO}-\mathrm{d}_{6} ;+=\right.$ $\mathrm{H}_{2} \mathrm{O} ; \mathbf{x}=\mathrm{CH}_{2} \mathrm{Cl}_{2}$ ). Two species are observed, which are in a solvent- and temperature-dependent equilibrium with one another; see Figure S59.

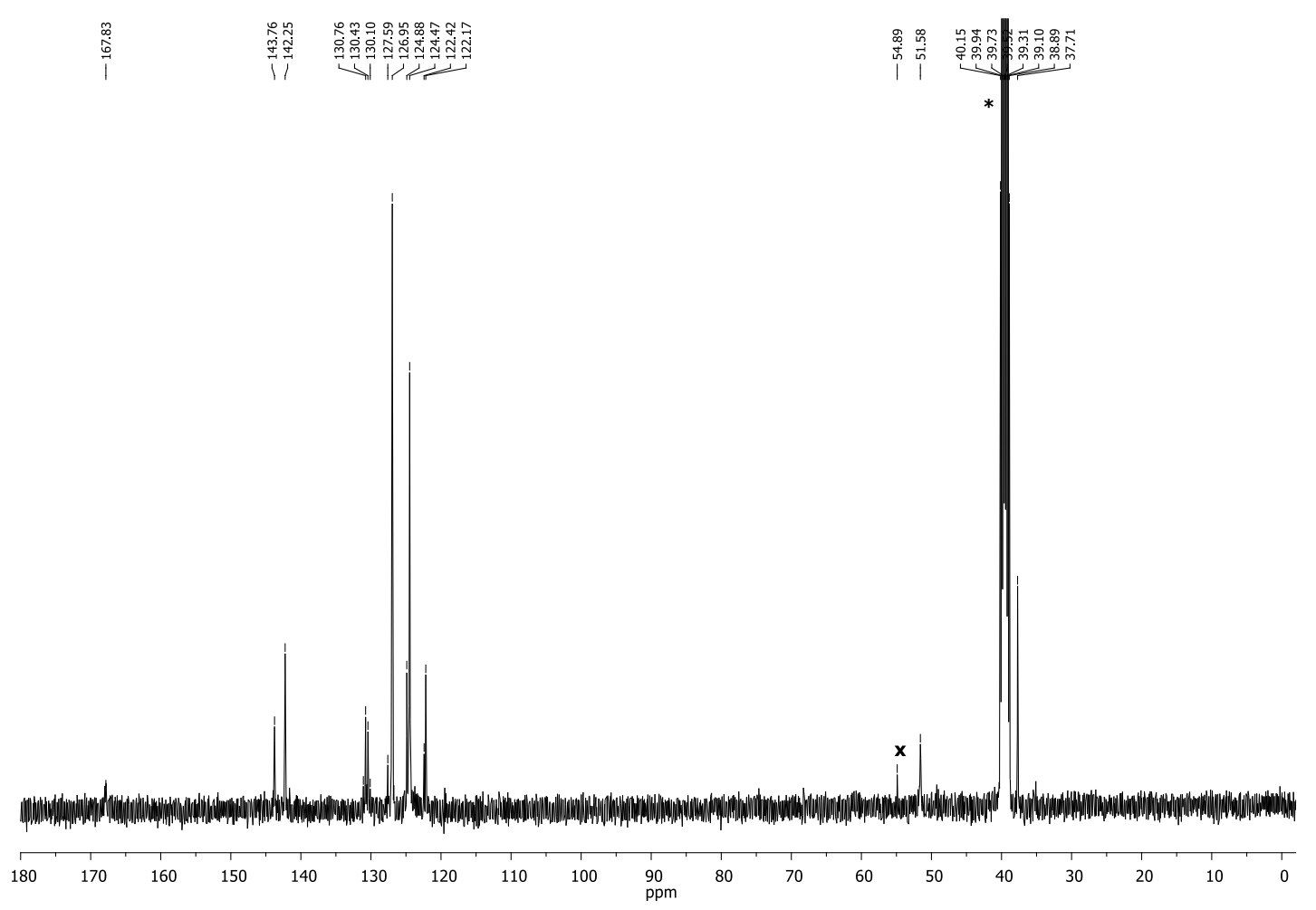

Figure S56. ${ }^{13} \mathrm{C}$ NMR spectrum of $\left[\mathrm{Ag}_{3}(\mathbf{1} \mathbf{b})_{2}\right]\left(\mathrm{BF}_{4}\right)_{3}$, recorded in DMSO- $\mathrm{d}_{6}$ solution $\left(*=\mathrm{DMSO}-\mathrm{d}_{6} ; \mathbf{x}=\right.$ $\mathrm{CH}_{2} \mathrm{Cl}_{2}$ ). 


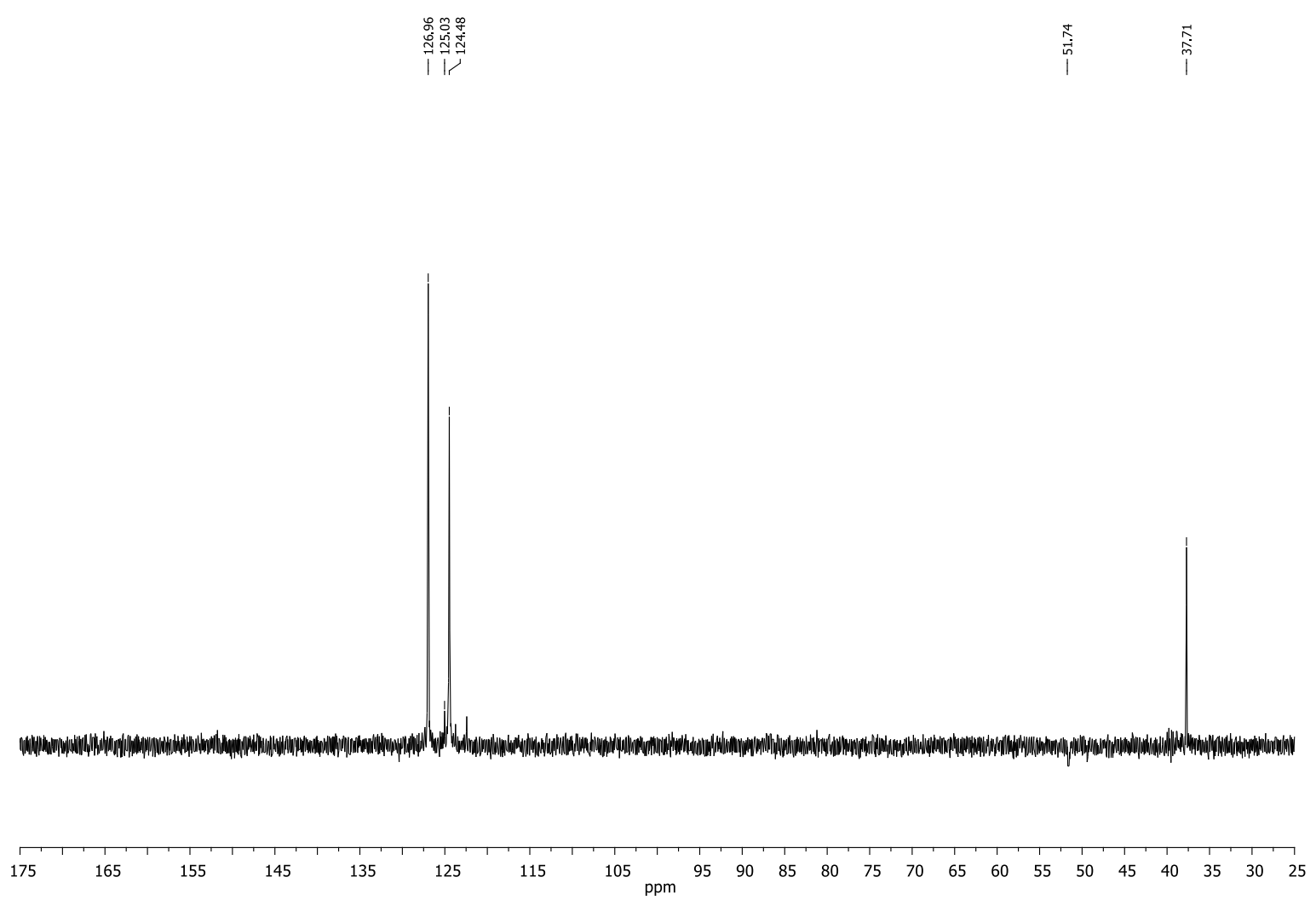

Figure S57. DEPT-135 NMR spectrum of $\left[\mathrm{Ag}_{3}(\mathbf{1} \mathbf{b})_{2}\right]\left(\mathrm{BF}_{4}\right)_{3}$, recorded in DMSO-d $\mathrm{d}_{6}$ solution.

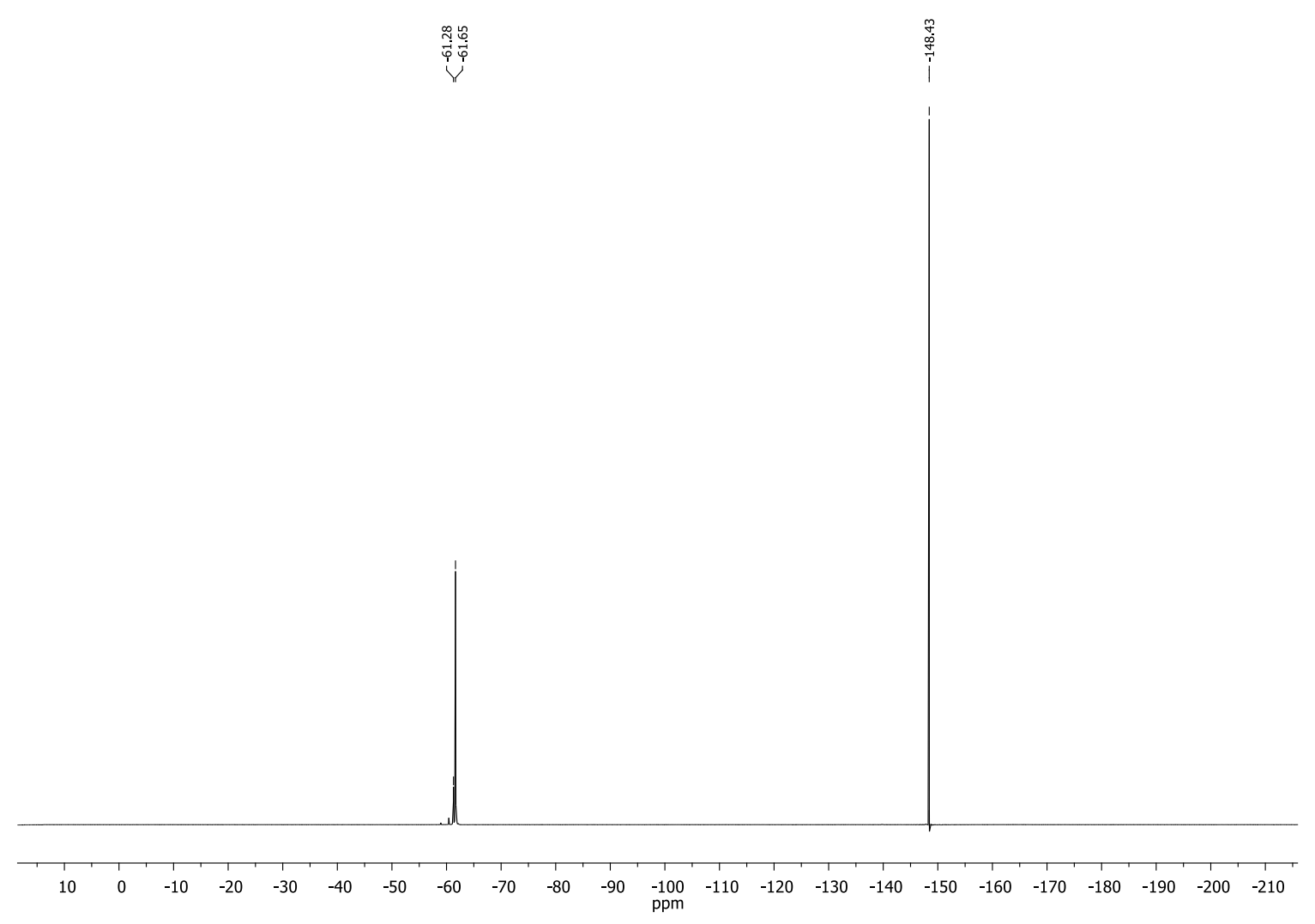

Figure S58. ${ }^{19} \mathrm{~F}$ NMR spectrum of $\left[\mathrm{Ag}_{3}(\mathbf{1} \mathbf{b})_{2}\right]\left(\mathrm{BF}_{4}\right)_{3}$, recorded in DMSO-d 6 solution. 


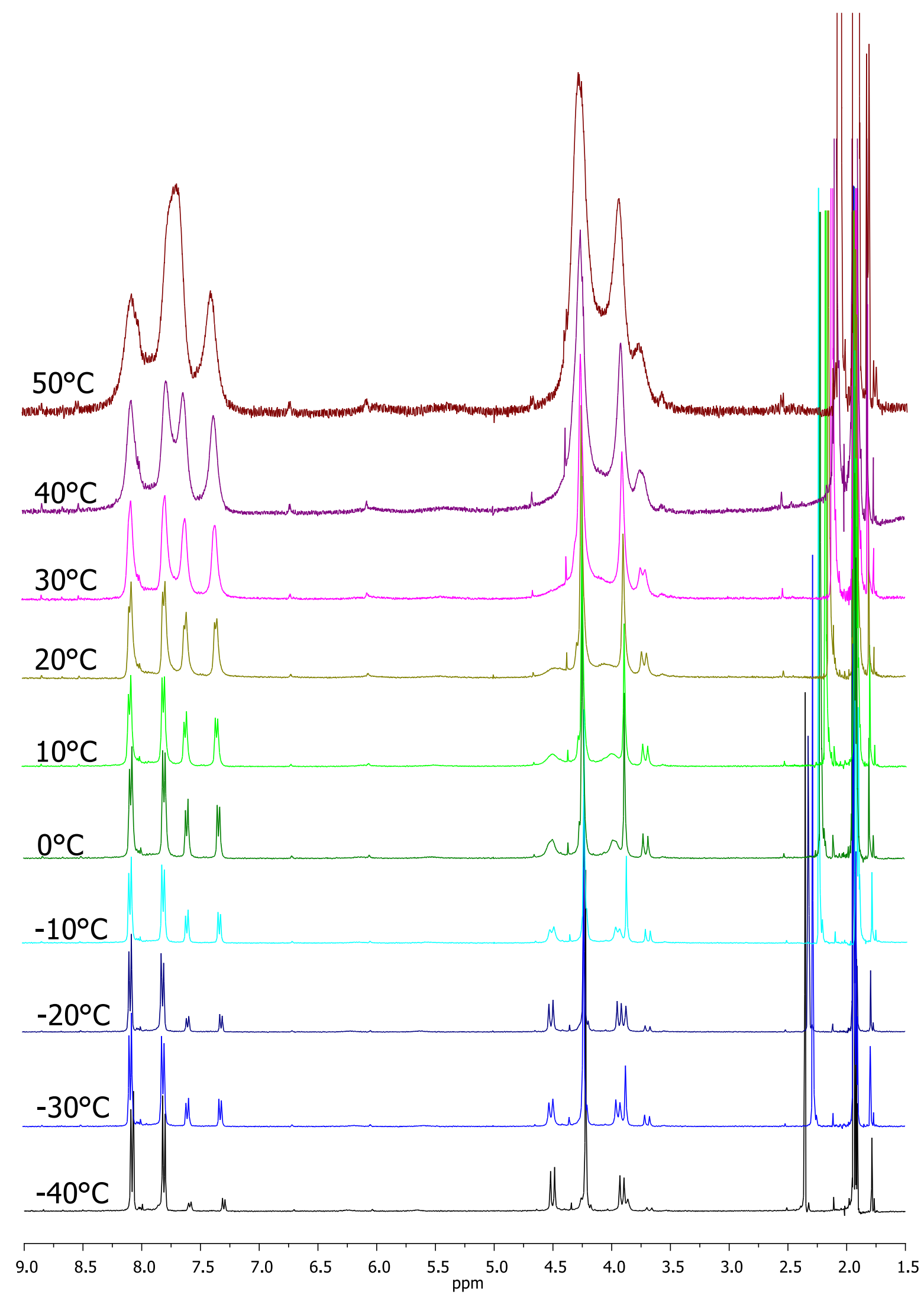

Figure S59. ${ }^{1} \mathrm{H}$ NMR spectrum of $\left[\mathrm{Ag}_{3}(\mathbf{1 b})_{2}\right]\left(\mathrm{BF}_{4}\right)_{3}$, recorded in $\mathrm{CD}_{3} \mathrm{CN}$ solution from $-40{ }^{\circ} \mathrm{C}$ to $50{ }^{\circ} \mathrm{C}$. 


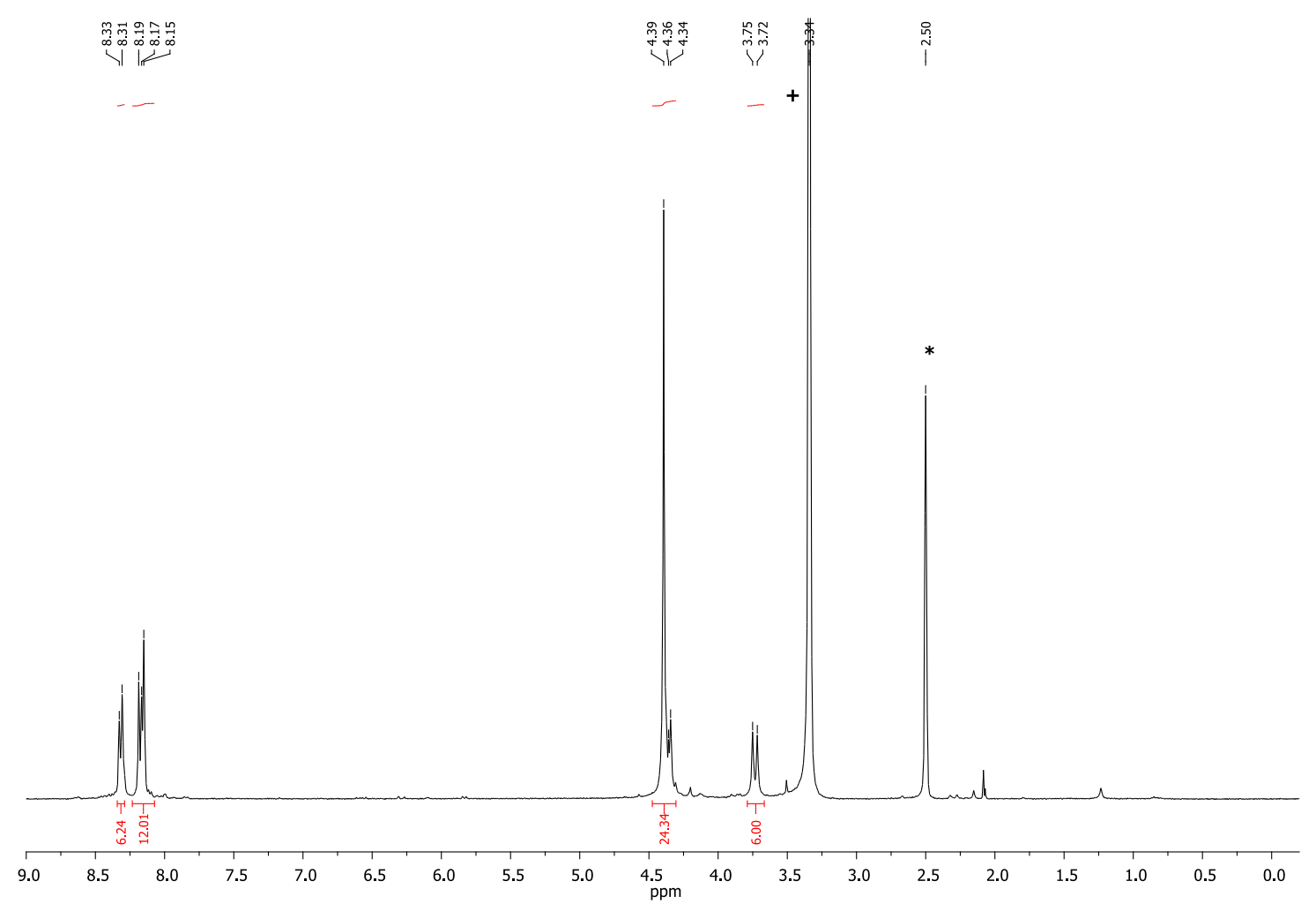

Figure S60. ${ }^{1} \mathrm{H}$ NMR spectrum of $\left[\mathrm{Ag}_{3}(\mathbf{1 c})_{2}\right]\left(\mathrm{BF}_{4}\right)_{3}$, recorded DMSO- $\mathrm{d}_{6}$ solution $\left(*=\mathrm{DMSO}-\mathrm{d}_{6} ;+=\mathrm{H}_{2} \mathrm{O}\right)$.

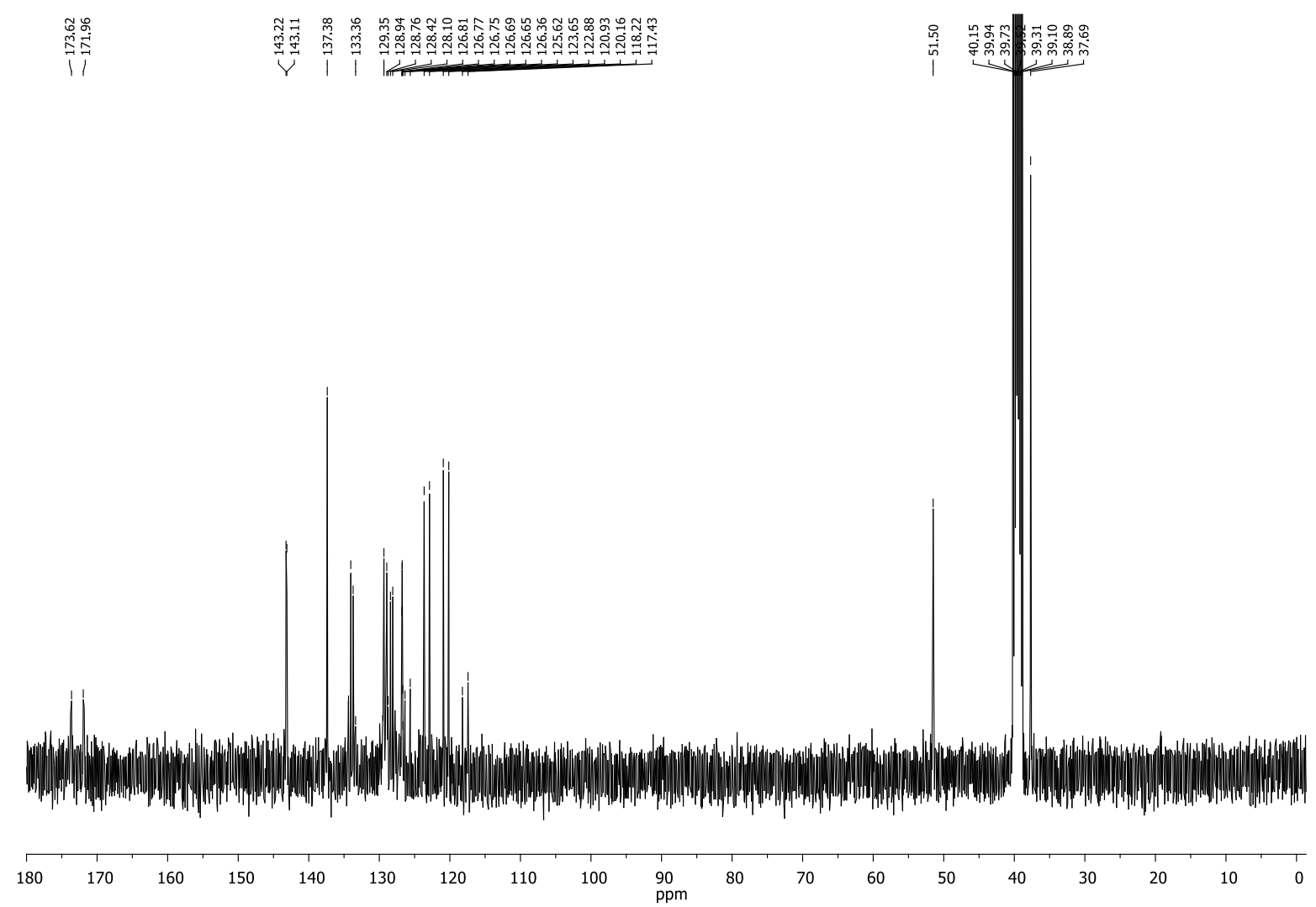

Figure S61. ${ }^{13} \mathrm{C}$ NMR spectrum of $\left[\mathrm{Ag}_{3}(\mathbf{1 c})_{2}\right]\left(\mathrm{BF}_{4}\right)_{3}$, recorded in DMSO-d $6\left({ }^{*}\right)$ solution. 


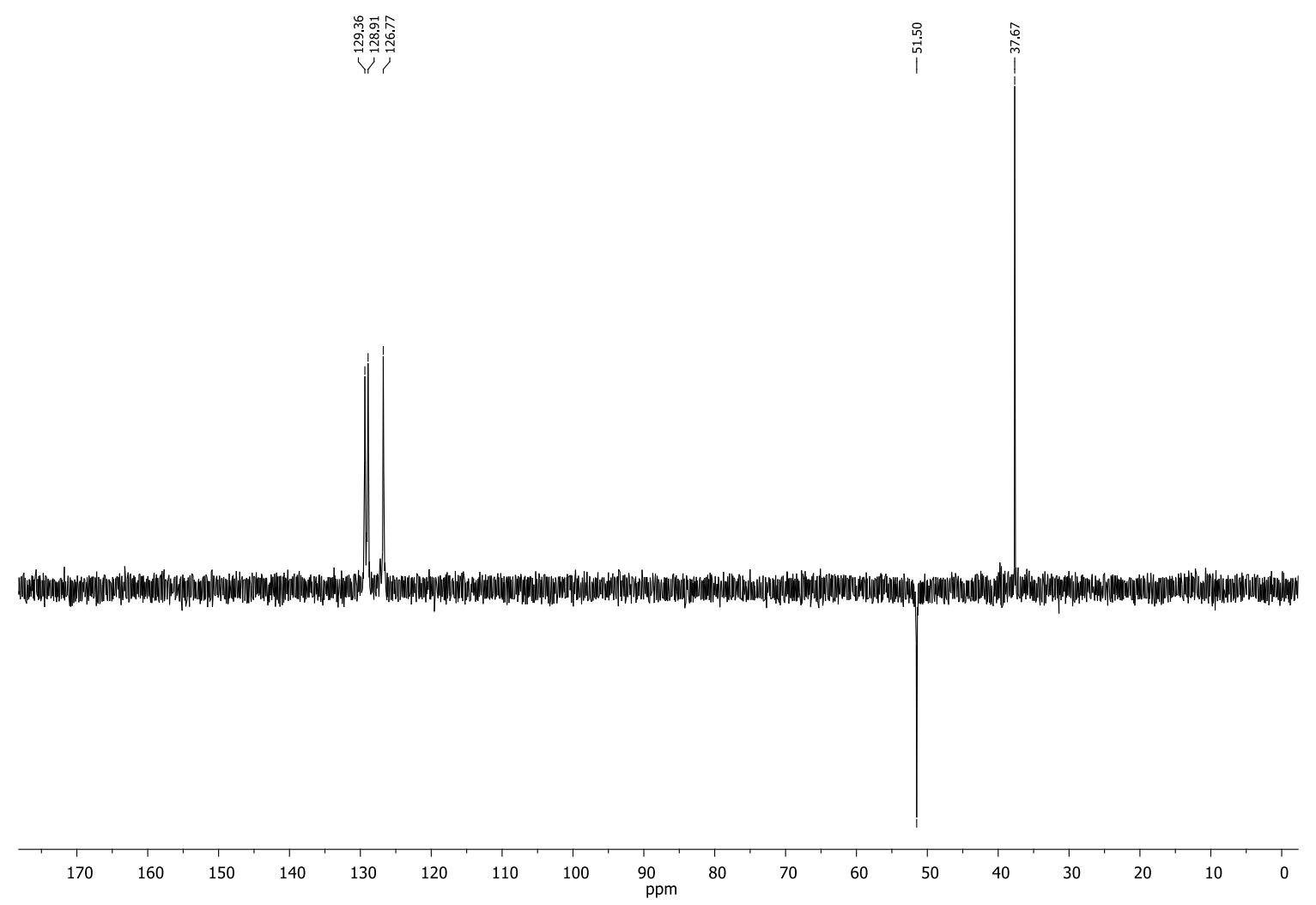

Figure S62. DEPT-135 NMR spectrum of $\left[\mathrm{Ag}_{3}(1 \mathrm{c})_{2}\right]\left(\mathrm{BF}_{4}\right)_{3}$, recorded in DMSO-d $\mathrm{d}_{6}$ solution.

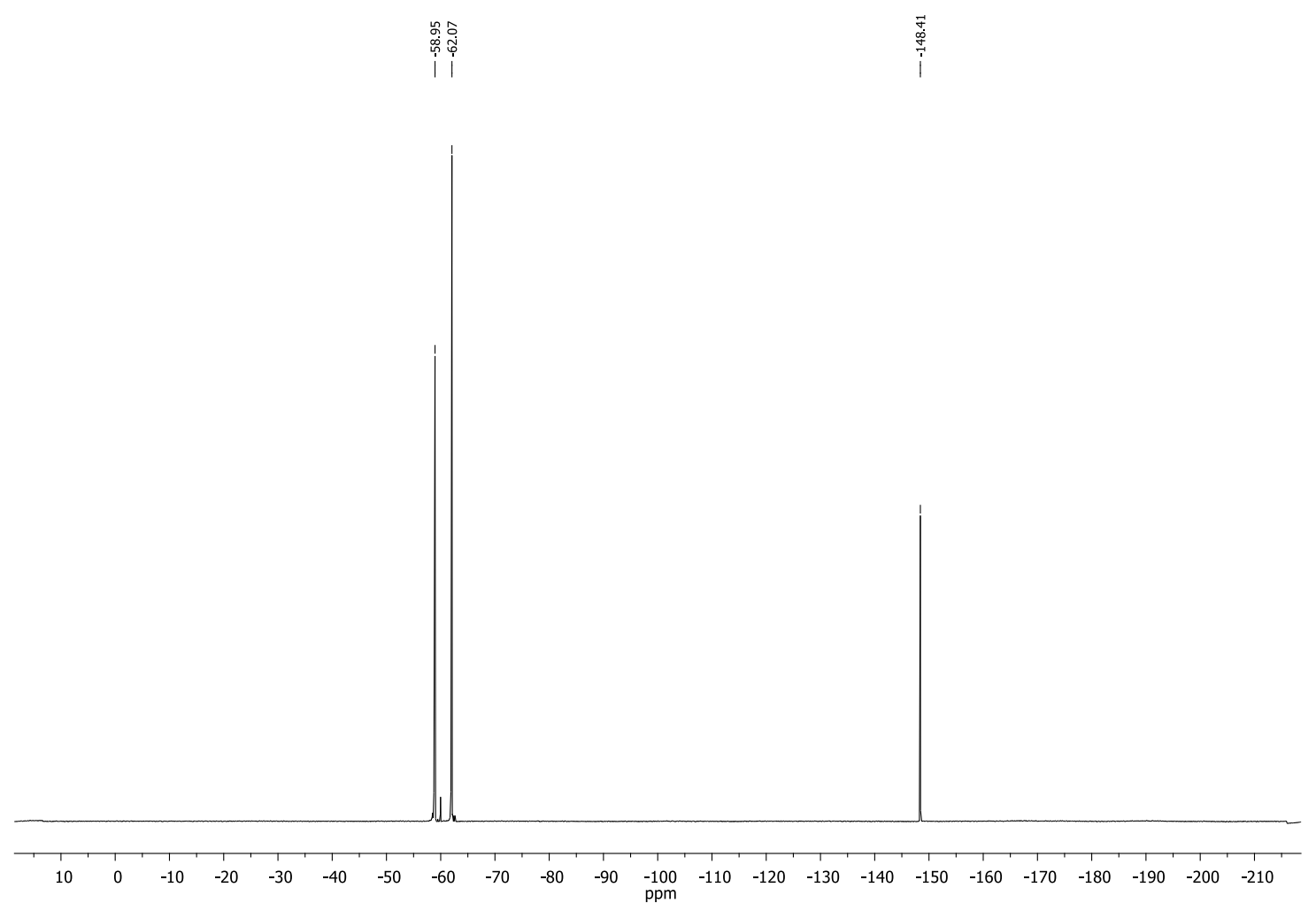

Figure S63. ${ }^{19} \mathrm{~F}$ NMR spectrum of $\left[\mathrm{Ag}_{3}(1 \mathrm{c})_{2}\right]\left(\mathrm{BF}_{4}\right)_{3}$, recorded in DMSO-d $\mathrm{d}_{6}$ solution. 


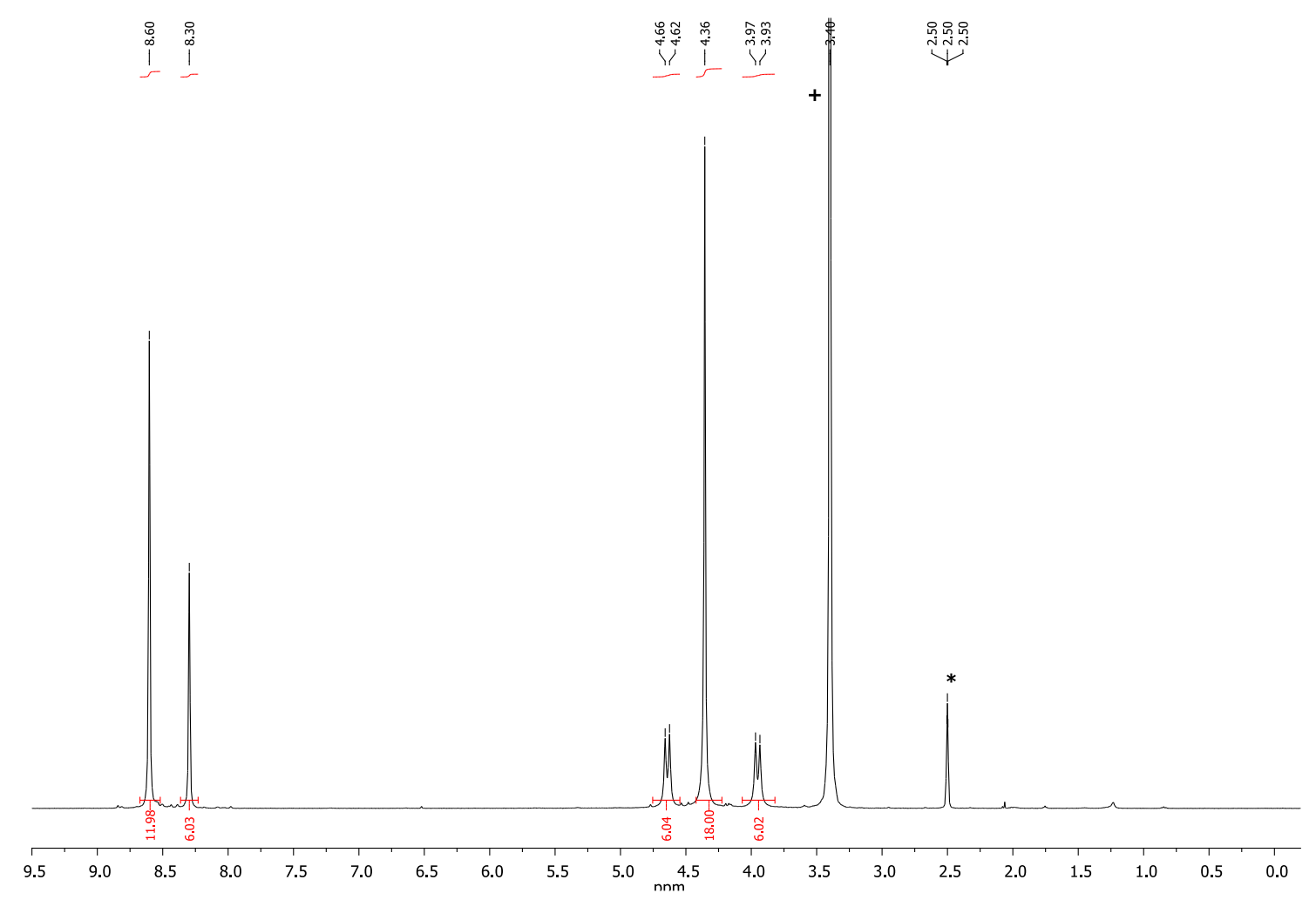

Figure S64. ${ }^{1} \mathrm{H}$ NMR spectrum of $\left[\mathrm{Ag}_{3}(\mathbf{1 d})_{2}\right]\left(\mathrm{BF}_{4}\right)_{3}$, recorded in DMSO- $\mathrm{d}_{6}$ solution $\left({ }^{*}=\mathrm{DMSO}-\mathrm{d}_{6} ;+=\right.$ $\mathrm{H}_{2} \mathrm{O}$ ).

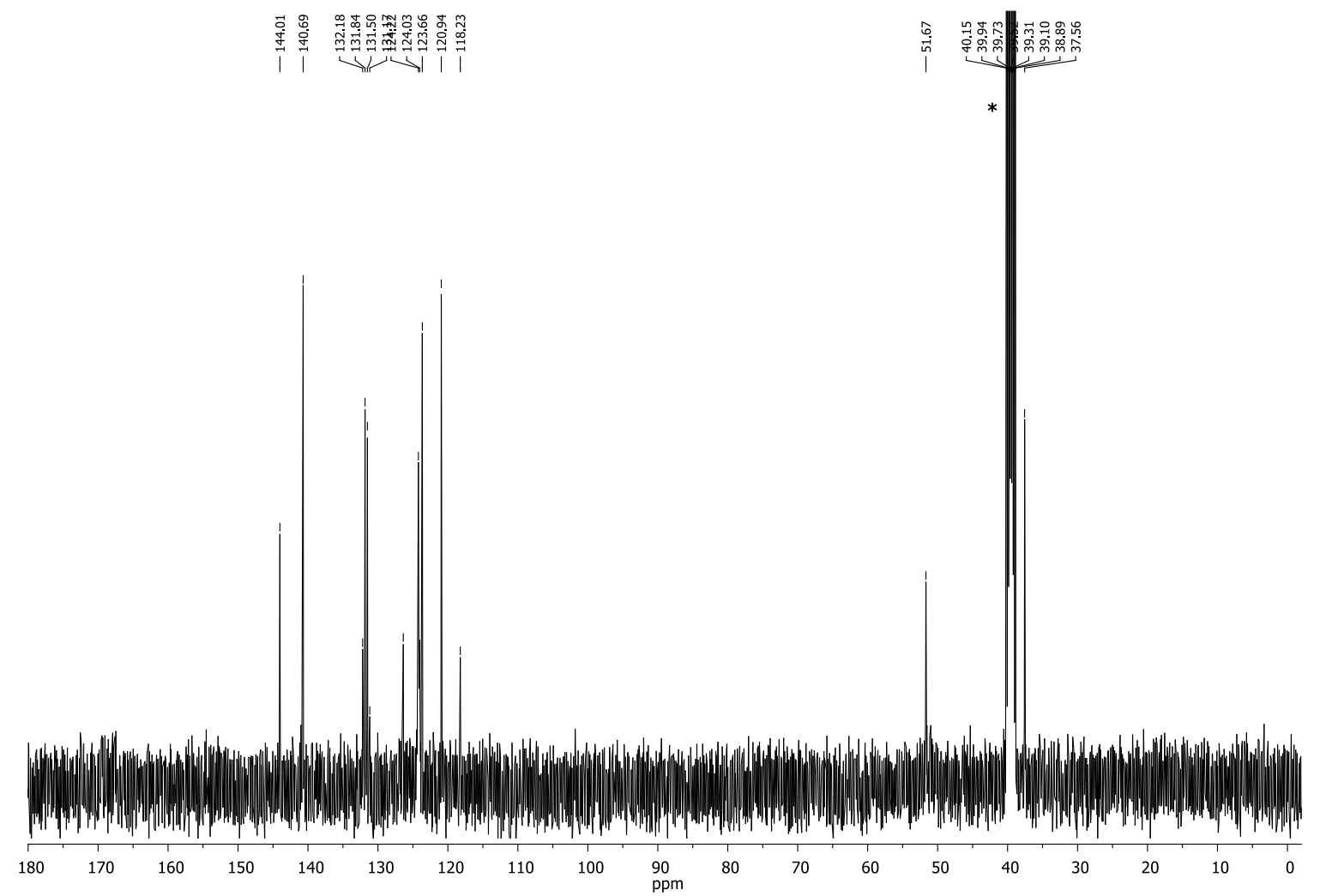

Figure S65. ${ }^{13} \mathrm{C}$ NMR spectrum of $\left[\mathrm{Ag}_{3}(\mathbf{1 d})_{2}\right]\left(\mathrm{BF}_{4}\right)_{3}$, recorded in DMSO-d $6\left(^{*}\right)$ solution. 


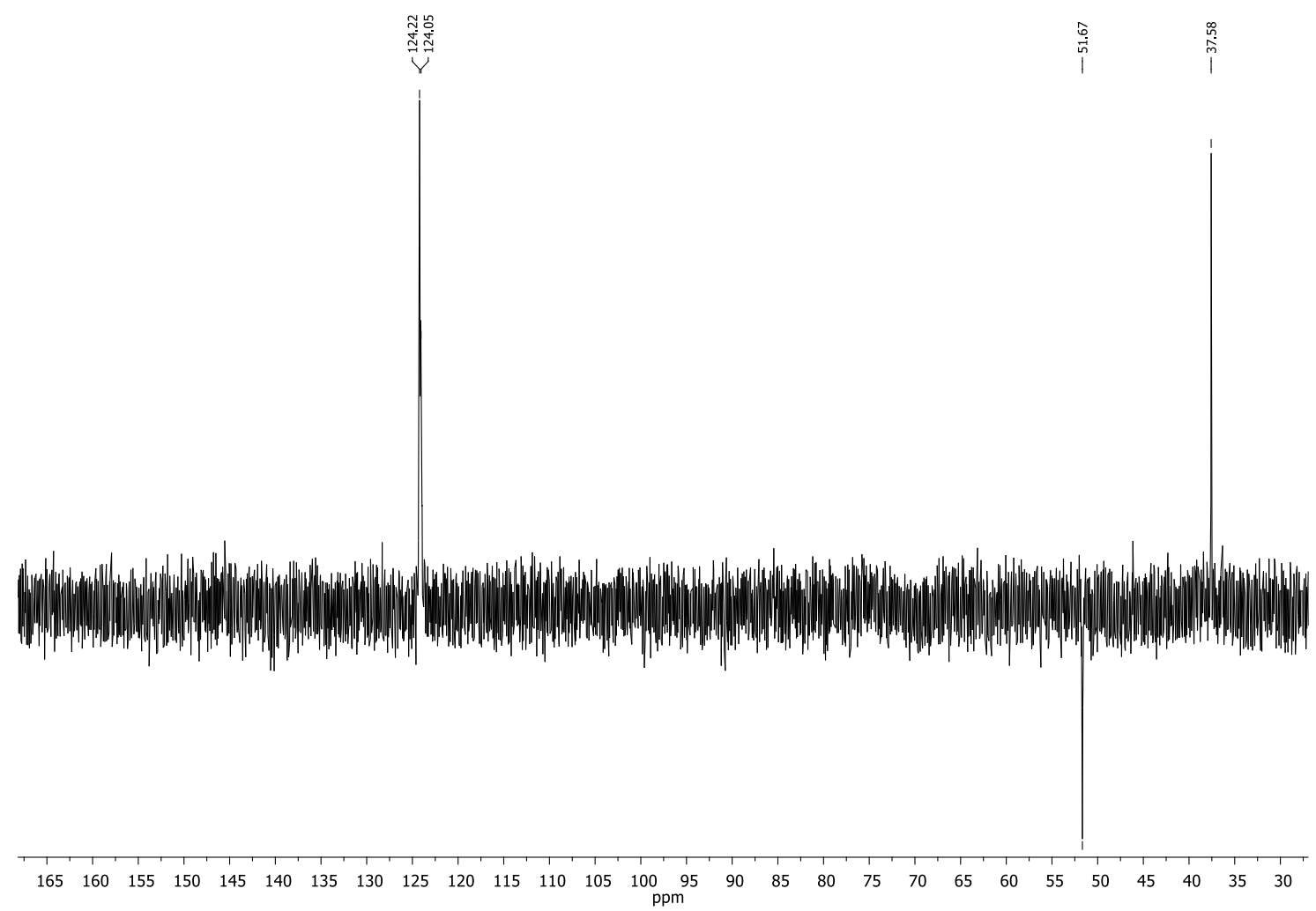

Figure S66. DEPT-135 NMR spectrum of $\left[\mathrm{Ag}_{3}(\mathbf{1 d})_{2}\right]\left(\mathrm{BF}_{4}\right)_{3}$, recorded in DMSO- $\mathrm{d}_{6}$ solution.

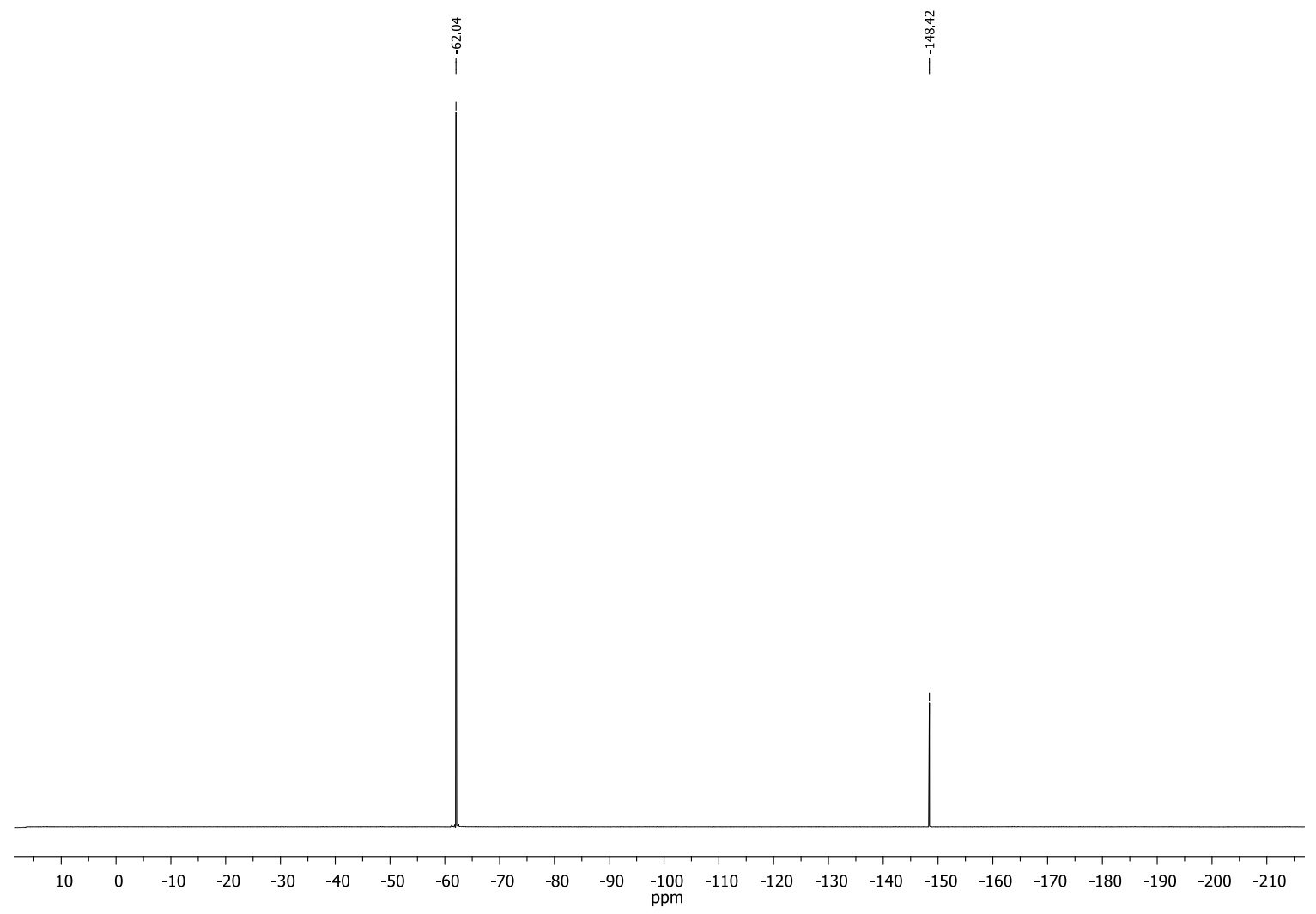

Figure S67. ${ }^{19} \mathrm{~F} N M R$ spectrum of $\left[\mathrm{Ag}_{3}(1 \mathrm{~d})_{2}\right]\left(\mathrm{BF}_{4}\right)_{3}$, recorded in DMSO- $\mathrm{d}_{6}$ solution. 


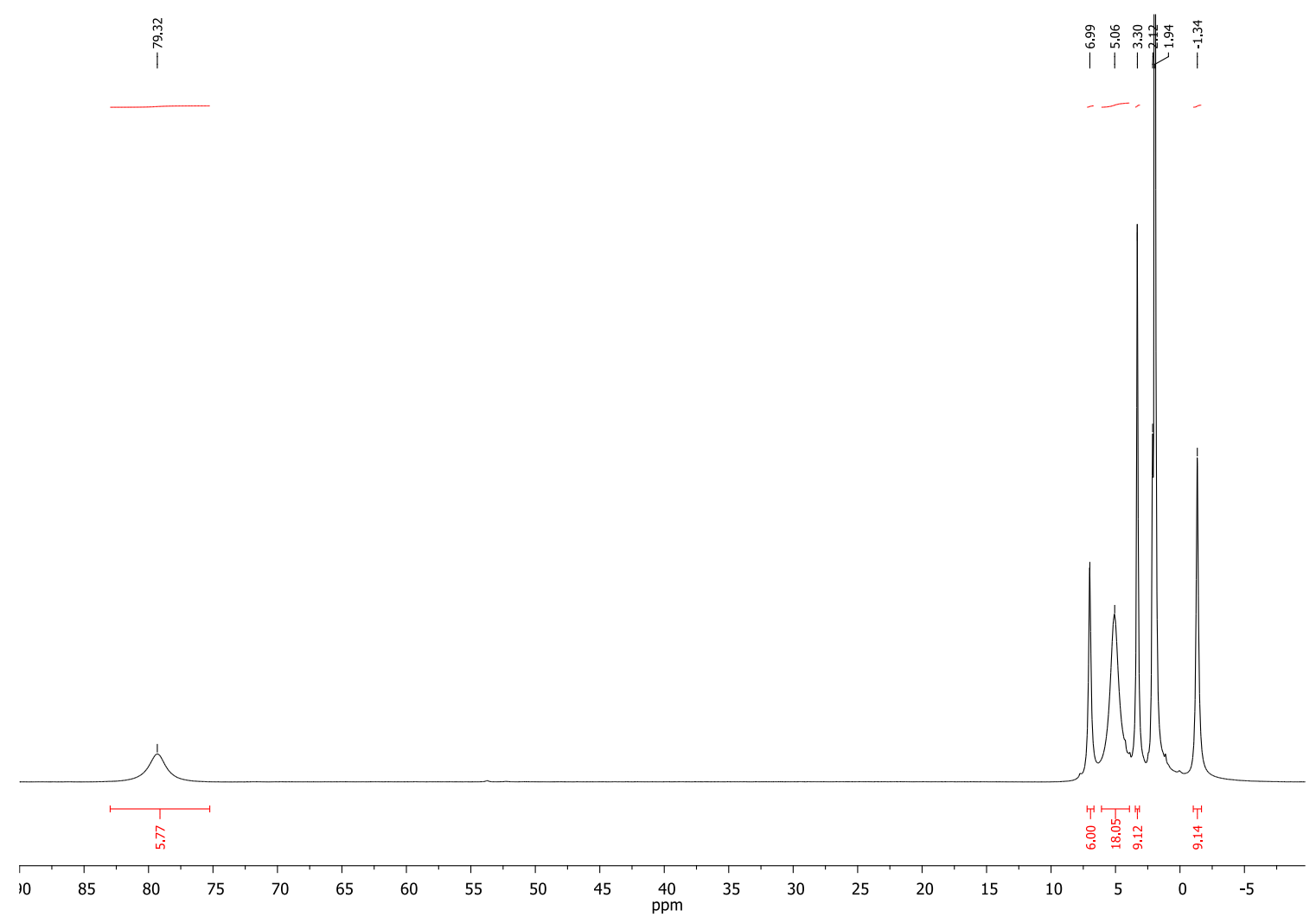

Figure S68. ${ }^{1} \mathrm{H} N M R$ spectrum of $[\mathrm{Co}(1 \mathrm{a})(\mathrm{MeCN})](\mathrm{OTf})_{2}$, recorded in $\mathrm{CD}_{3} \mathrm{CN}$ solution $\left({ }^{*}=\mathrm{CD}_{3} \mathrm{CN}\right)$.

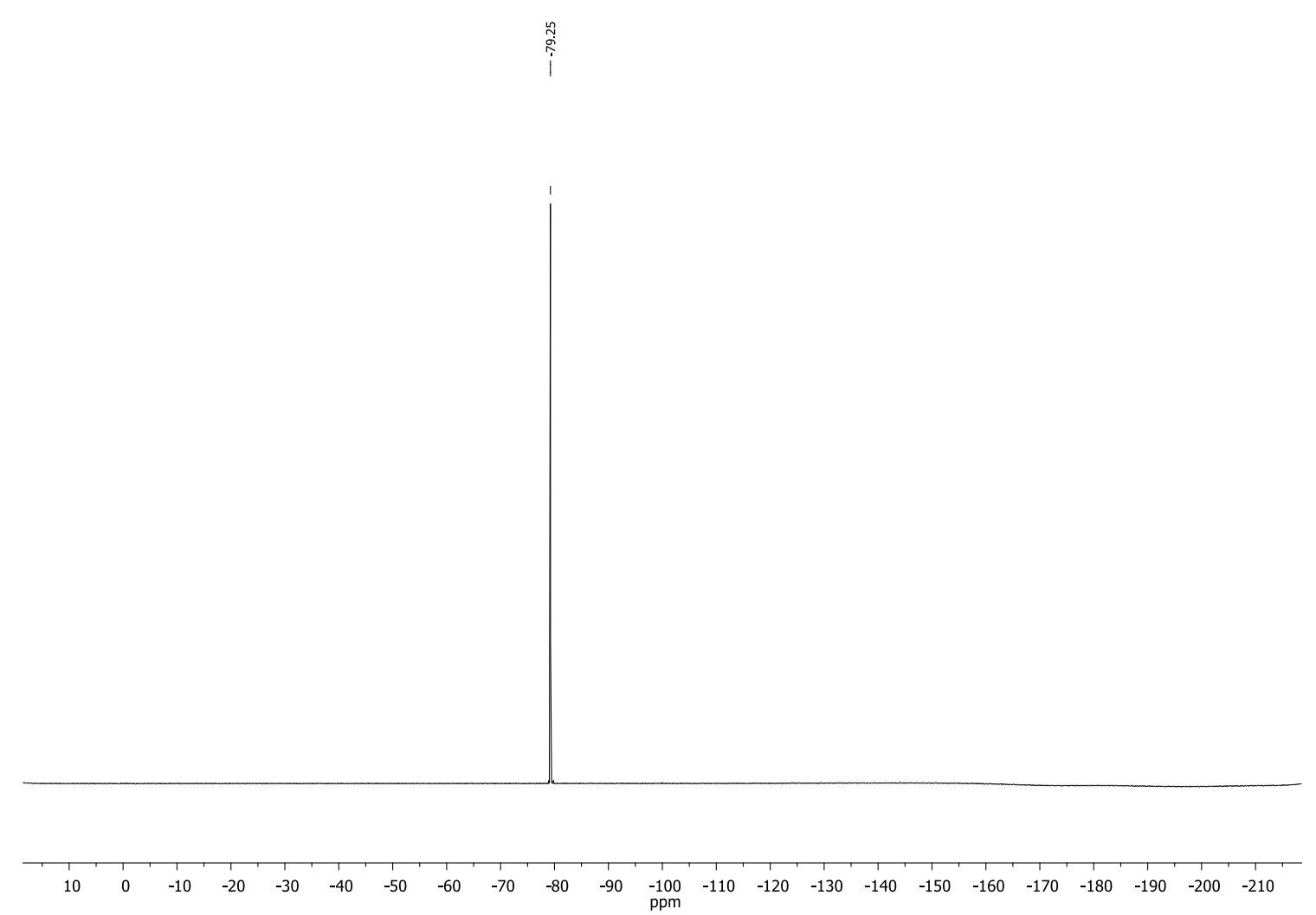

Figure S69. ${ }^{19} \mathrm{~F}$ NMR spectrum of $[\mathrm{Co}(1 \mathrm{a})(\mathrm{MeCN})](\mathrm{OTf})_{2}$, recorded in $\mathrm{CD}_{3} \mathrm{CN}$ solution. 


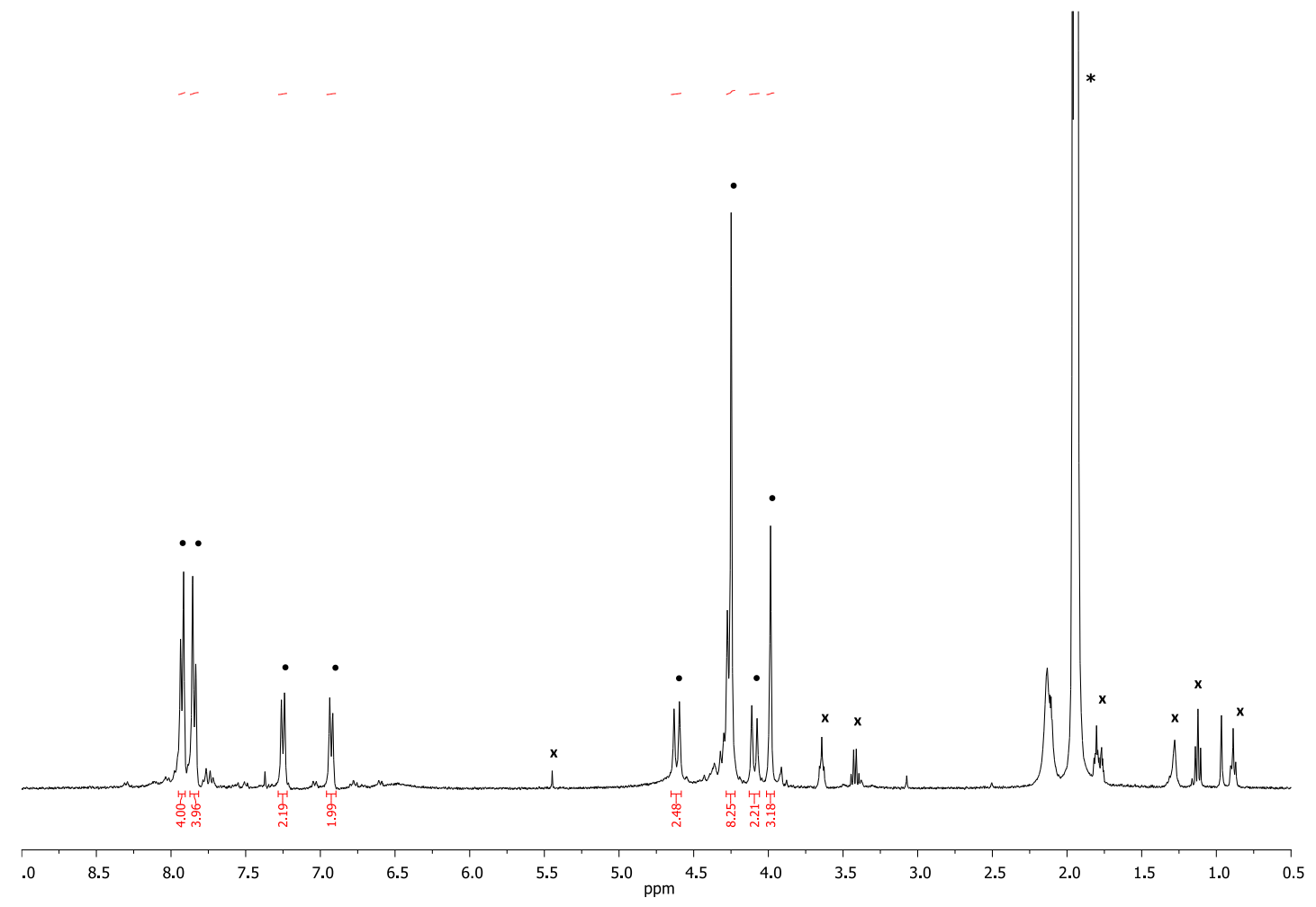

Figure S70. ${ }^{1} \mathrm{H}$ NMR spectrum of impure $[\mathrm{Co}(\mathbf{1 b})(\mathrm{Cl})(\mathrm{MeCN})](\mathrm{OTf})_{2}$ (denoted by $\bullet$ ), recorded in $\mathrm{CD}_{3} \mathrm{CN}$ solution ( ${ }^{*}=\mathrm{CD}_{3} \mathrm{CN}, \mathrm{x}=$ solvent).

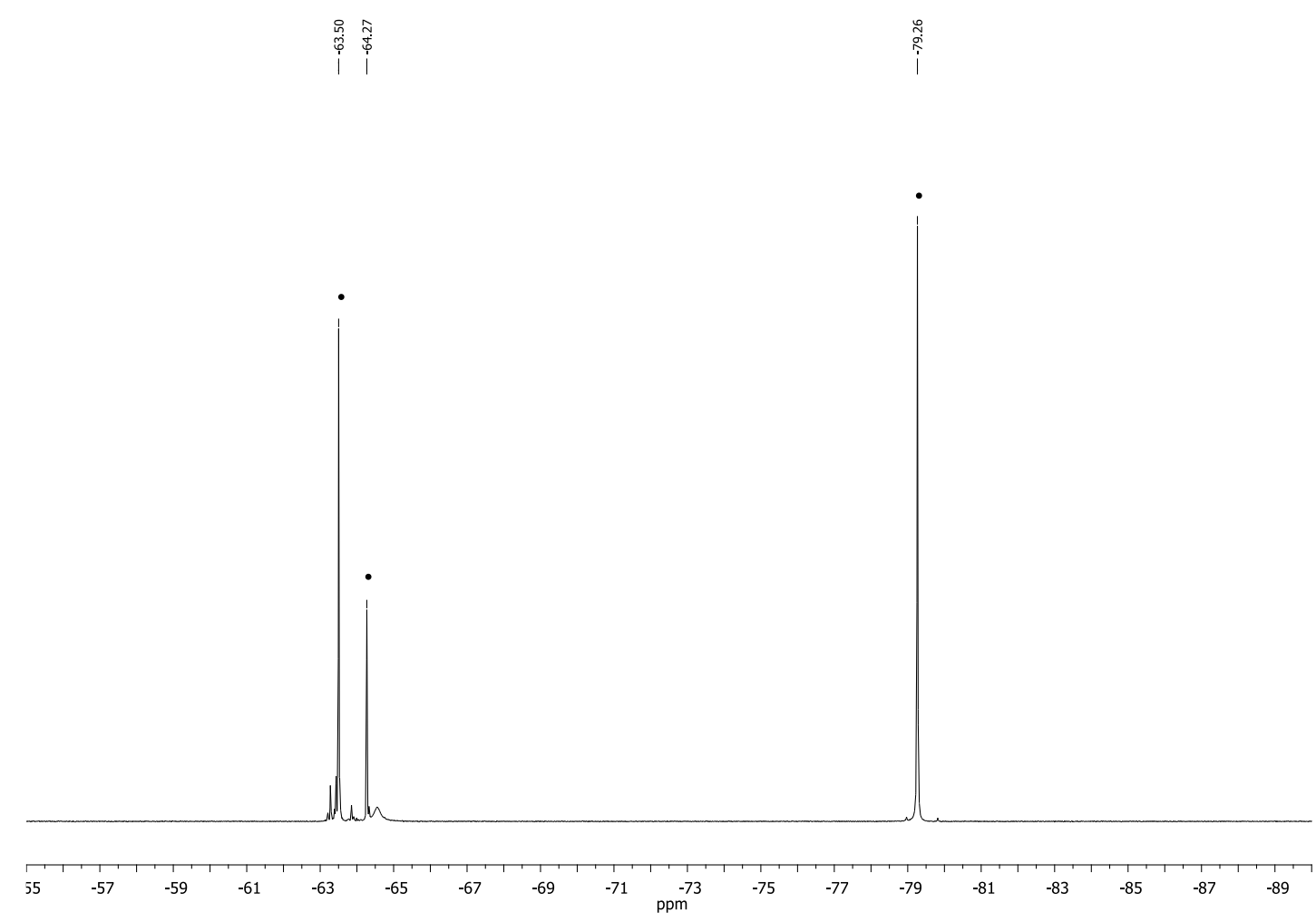

Figure S71. ${ }^{19} \mathrm{~F}$ NMR spectrum of impure $[\mathrm{Co}(\mathbf{1 b})(\mathrm{Cl})(\mathrm{MeCN})](\mathrm{OTf})_{2}($ denoted by $\bullet$ ), recorded in $\mathrm{CD}_{3} \mathrm{CN}$ solution. 


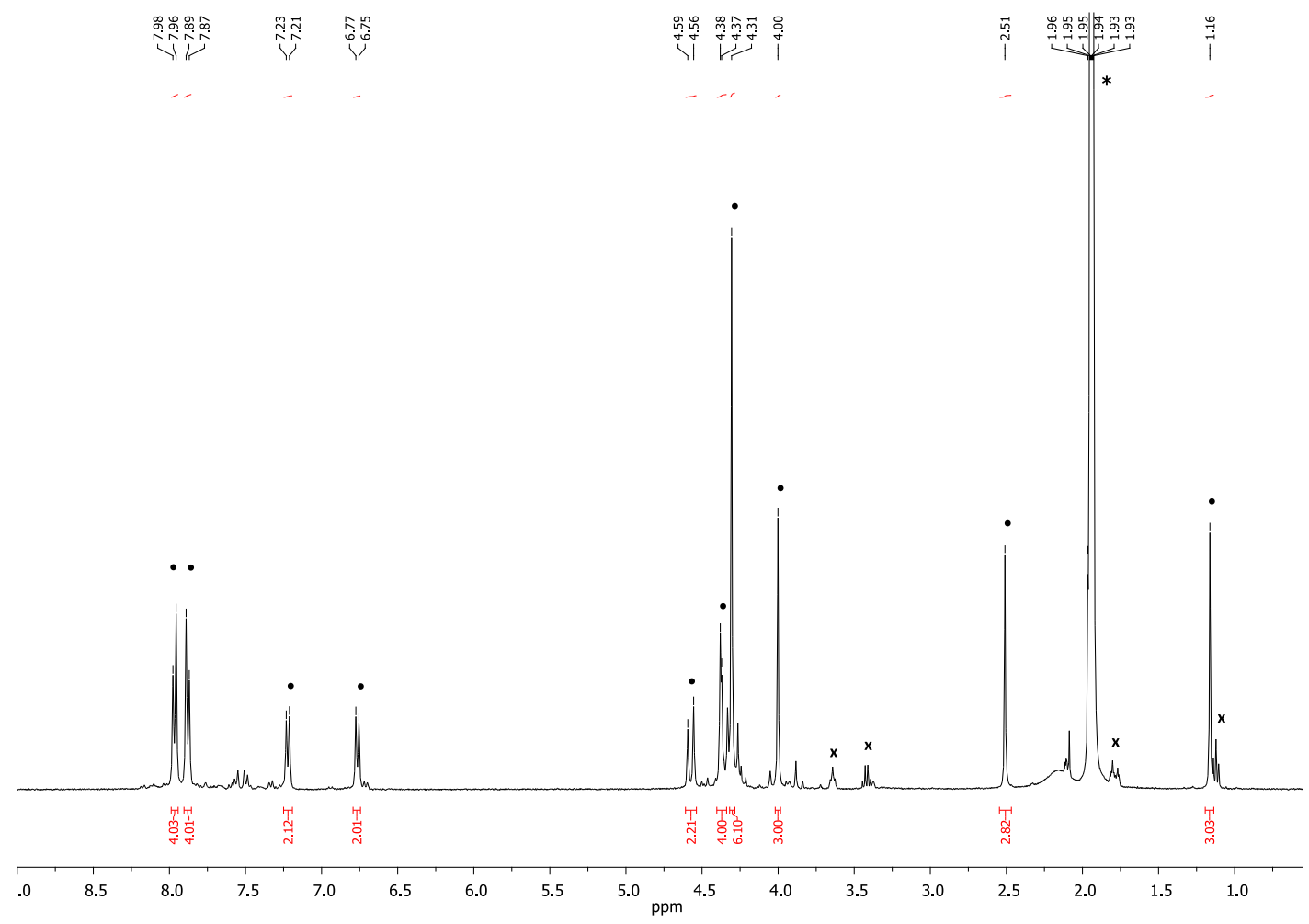

Figure S72. ${ }^{1} \mathrm{H}$ NMR spectrum of impure $\left[\mathrm{Co}(\mathbf{1 b})(\mathrm{MeCN})_{2}\right](\mathrm{OTf})_{3}$ (denoted by $\bullet$ ), recorded in $\mathrm{CD}_{3} \mathrm{CN}$ $\left(^{*}=\mathrm{CD}_{3} \mathrm{CN}, \mathrm{x}=\right.$ solvent $)$.

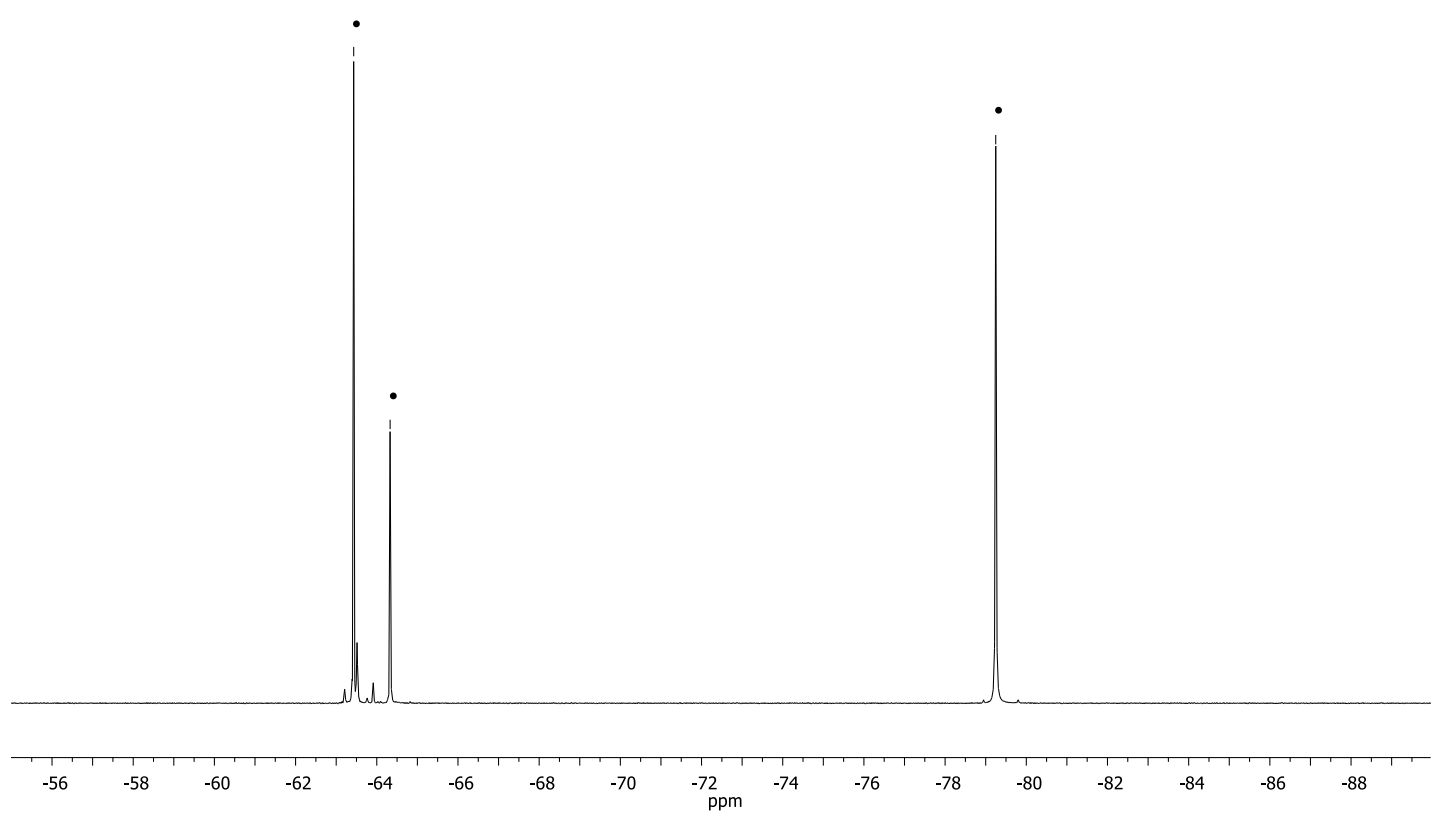

Figure S73. ${ }^{19} \mathrm{~F} \mathrm{NMR}$ spectrum of impure $\left[\mathrm{Co}(\mathbf{1 b})(\mathrm{MeCN})_{2}\right](\mathrm{OTf})_{3}$ (denoted by $\bullet$ ), recorded in $\mathrm{CD}_{3} \mathrm{CN}$ solution. 
Solution State vs Ball Milling

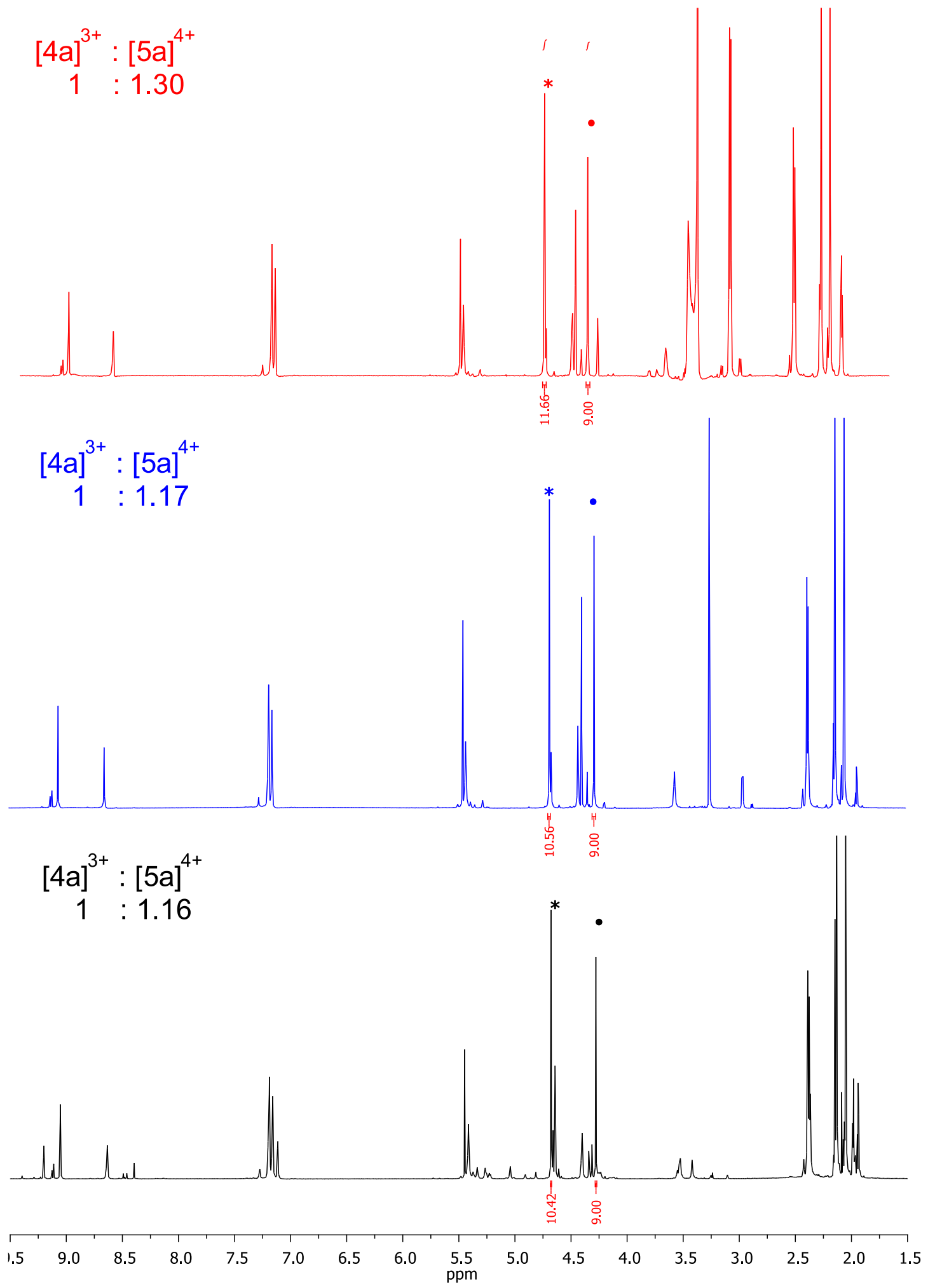

Figure S74. ${ }^{1} \mathrm{H}$ NMR spectra (recorded in $\mathrm{CD}_{3} \mathrm{CN}$ ) of the reaction mixtures obtained from reaction of 3a, in $\mathrm{CH}_{2} \mathrm{Cl}_{2}$ solution, with: 3.3 equiv $\mathrm{Me}_{3} \mathrm{OBF}_{4}$ (black); 4.5 equiv $\mathrm{Me}_{3} \mathrm{OBF}_{4}$ (blue); and 10.0 equiv $\mathrm{Me}_{3} \mathrm{OBF}_{4}$ (red). Triazolium methyl substituents: $[\mathbf{4 a}]^{3+}=\bullet ;[5 \mathbf{a}]^{4+}=*$. 


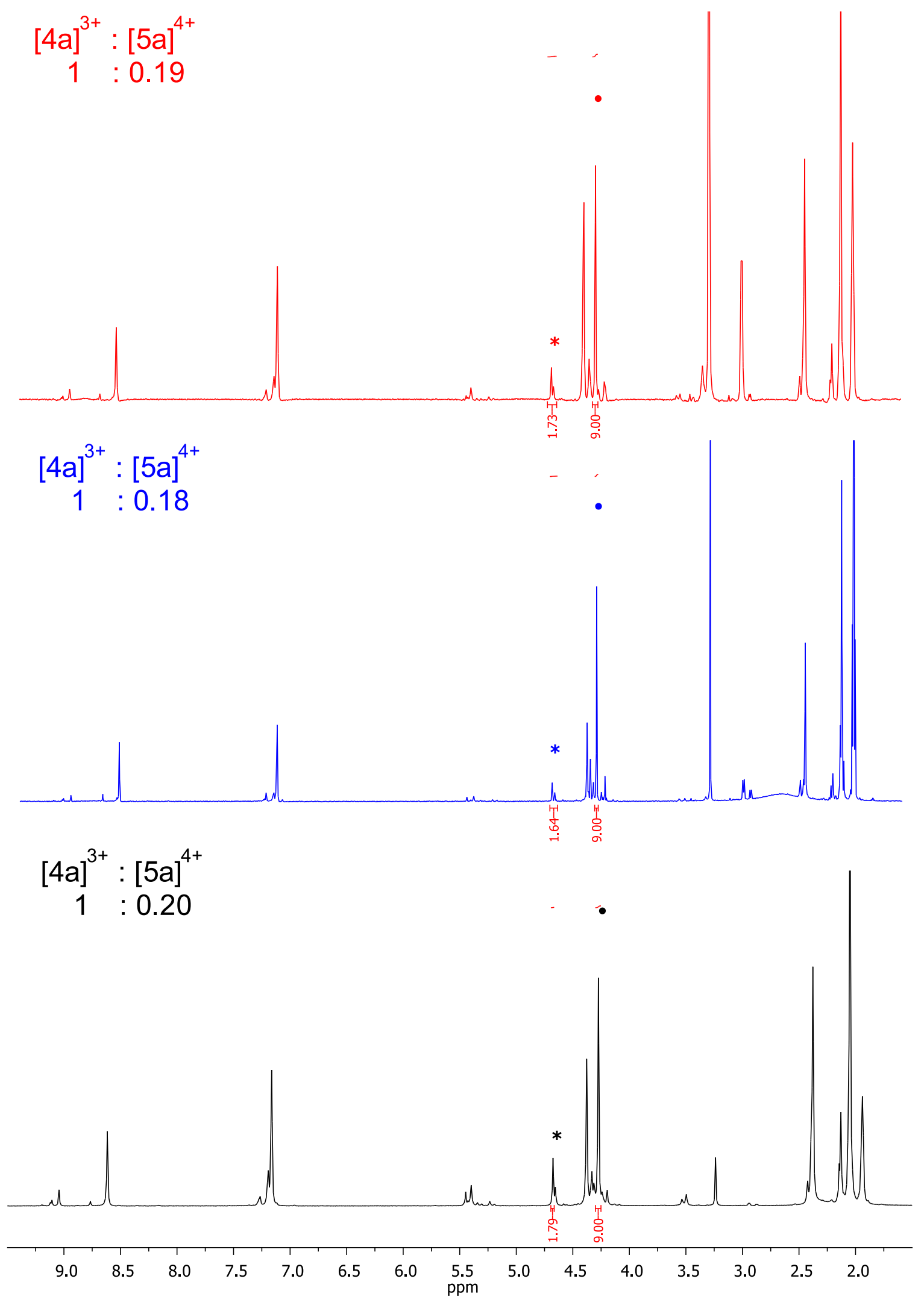

Figure S75. ${ }^{1} \mathrm{H}$ NMR spectra (recorded in $\mathrm{CD}_{3} \mathrm{CN}$ ) of the reaction mixtures obtained from ball milling 3a with: 3.3 equiv $\mathrm{Me}_{3} \mathrm{OBF}_{4}$ (black); 4.5 equiv $\mathrm{Me}_{3} \mathrm{OBF}_{4}$ (blue); and 6.0 equiv $\mathrm{Me}_{3} \mathrm{OBF}_{4}$ (red). Triazolium methyl substituents: $[\mathbf{4 a}]^{3+}=\bullet ;[\mathbf{5 a}]^{4+}=*$. 


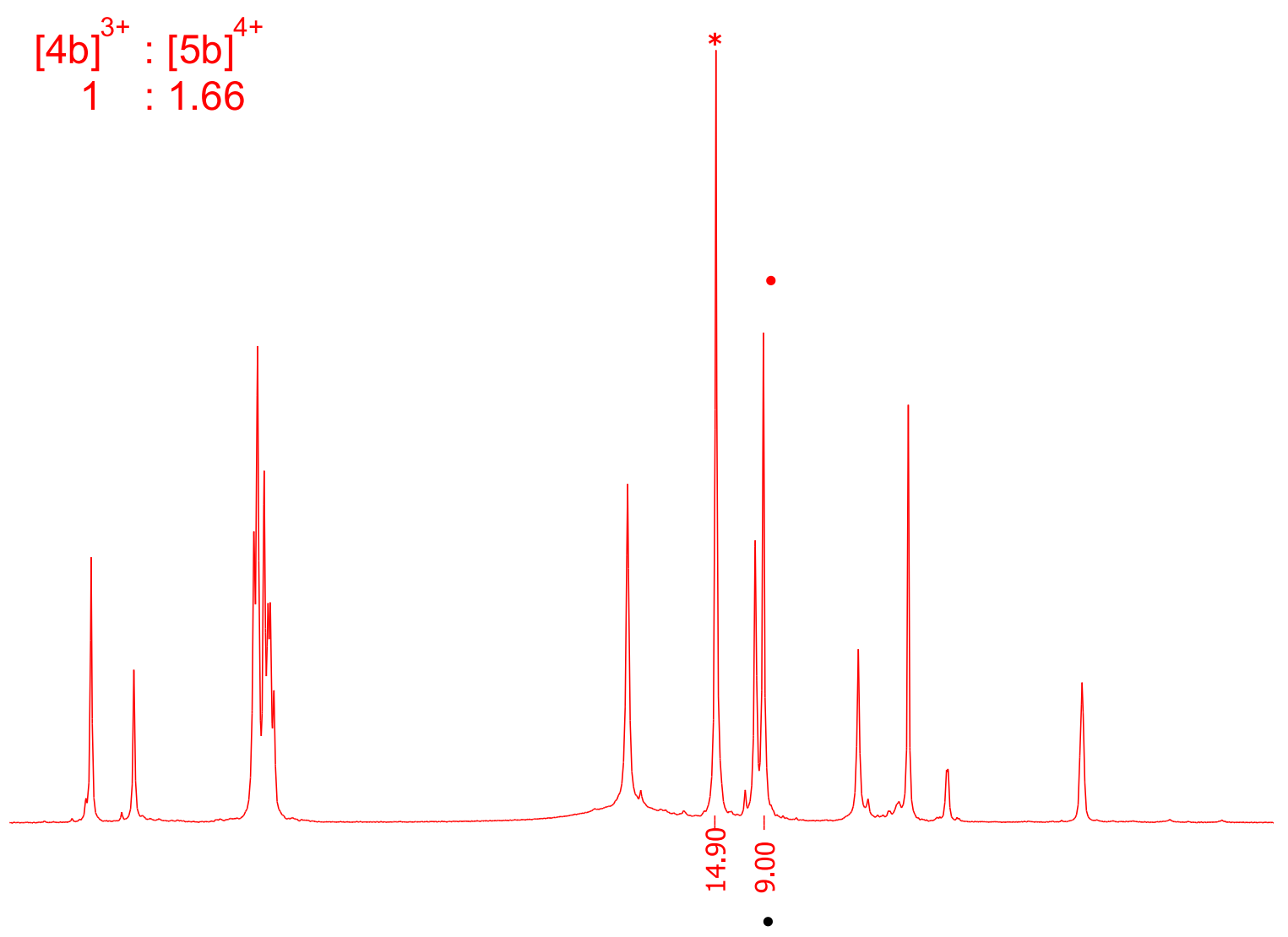

$$
\begin{aligned}
{[4 b]^{3+} } & :[5 b]^{4+} \\
1 & : 0.28
\end{aligned}
$$
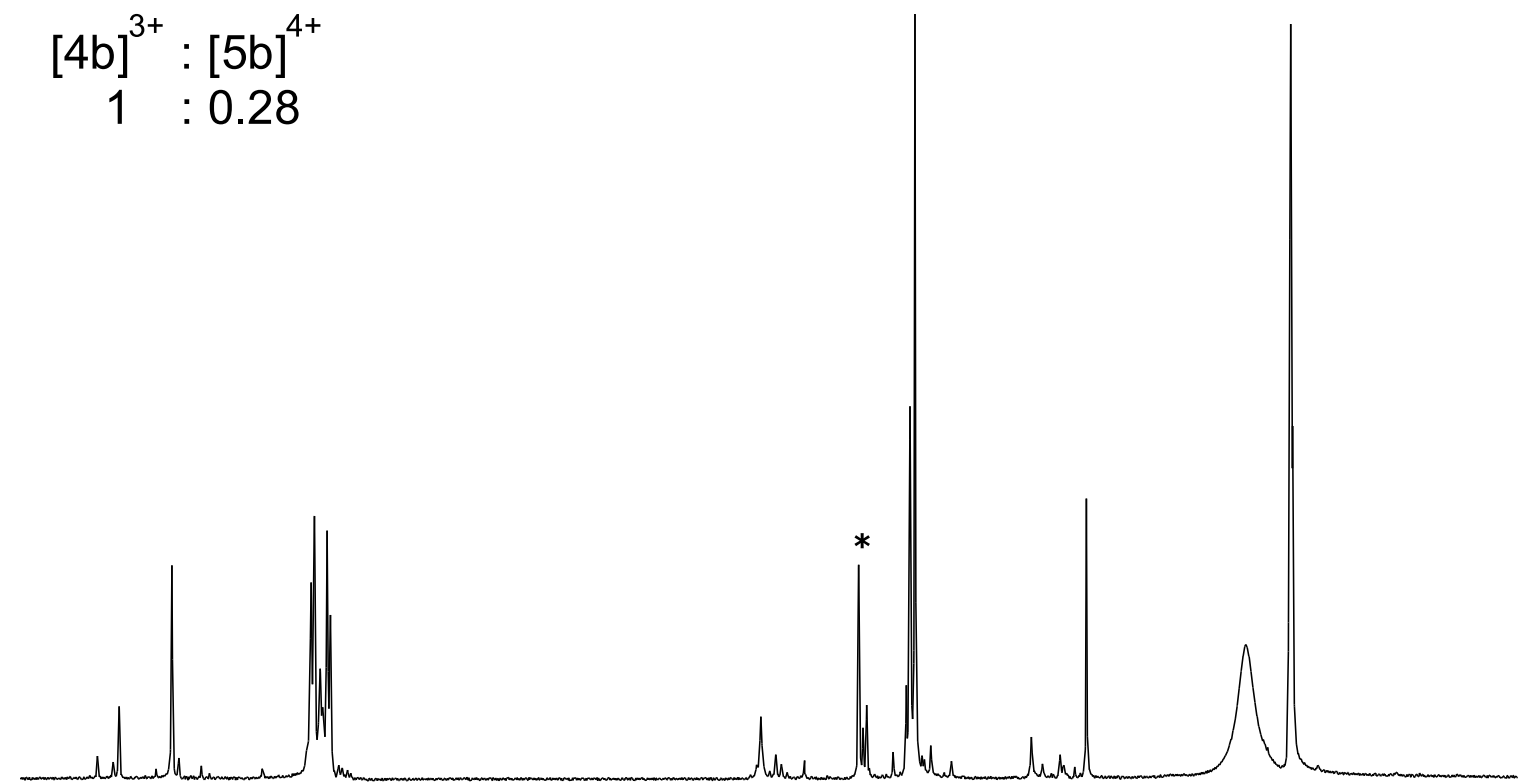

ํํํ \&े

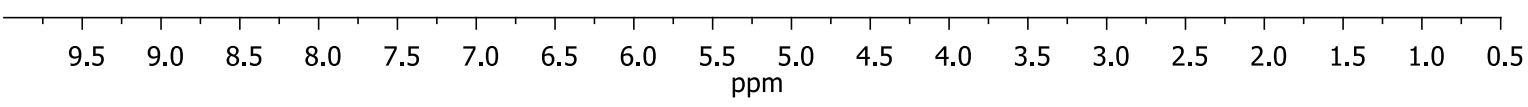

Figure S76. ${ }^{1} \mathrm{H}$ NMR spectra (recorded in $\mathrm{CD}_{3} \mathrm{CN}$ ) of the reaction mixtures obtained from reaction of 3b with $\mathrm{Me}_{3} \mathrm{OBF}_{4}$ in a $\mathrm{CH}_{2} \mathrm{Cl}_{2}$ solution (red) vs ball milling (black). Triazolium methyl substituents: [4b] $]^{3+}$ $=\bullet ;[5 b]^{4+}=$ *. 

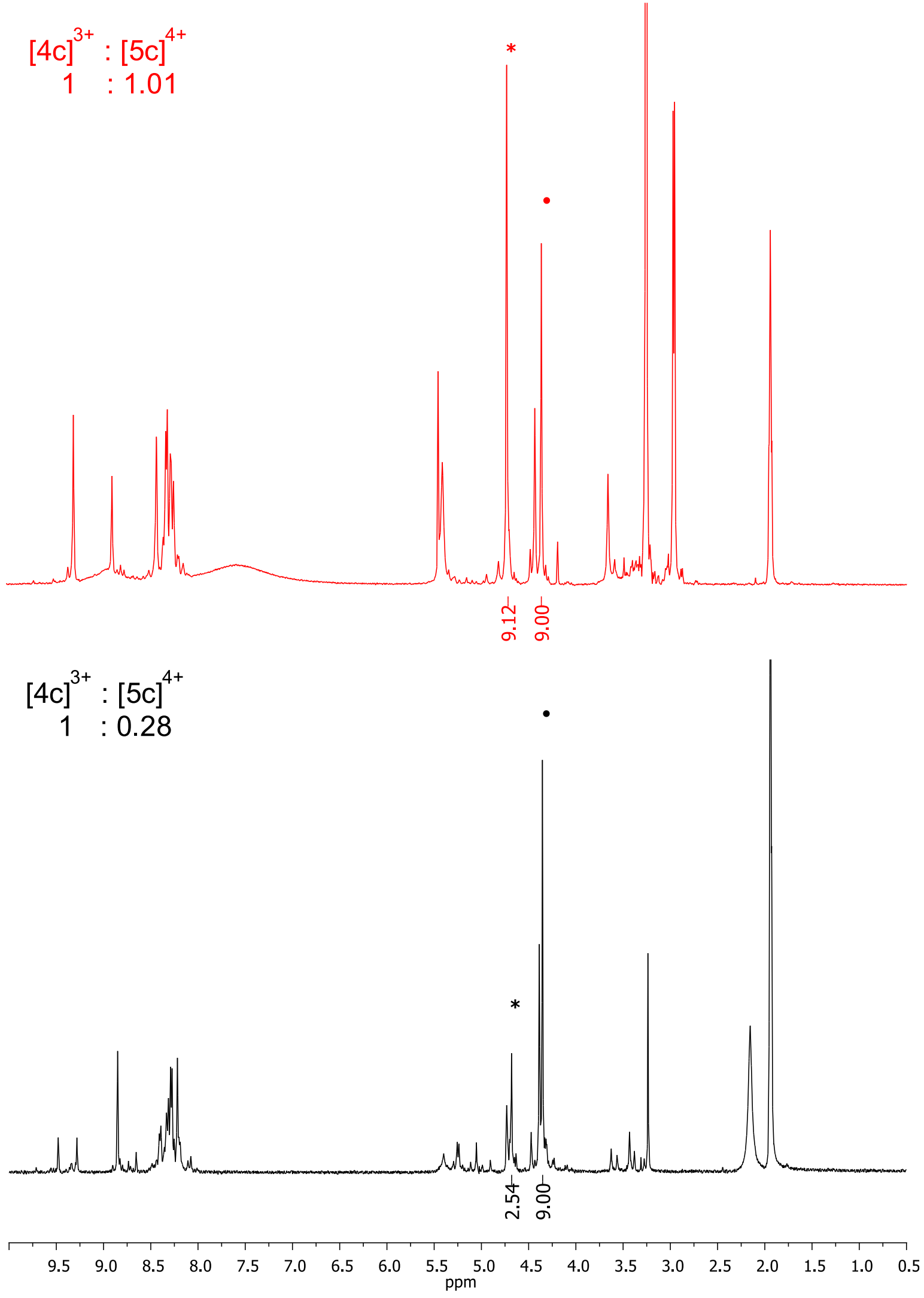

Figure S77. ${ }^{1} \mathrm{H}$ NMR spectra (recorded in $\mathrm{CD}_{3} \mathrm{CN}$ ) of the reaction mixtures obtained from reaction of 3c with $\mathrm{Me}_{3} \mathrm{OBF}_{4}$ in a $\mathrm{CH}_{2} \mathrm{Cl}_{2}$ solution (red) vs ball milling (black). Triazolium methyl substituents: [4c] $]^{3+}$ $=\bullet ;[5 \mathbf{c}]^{4+}=*$. 


$$
\begin{gathered}
{[4 d]^{3+}:[5 d]^{4+}} \\
1: 1.47
\end{gathered}
$$

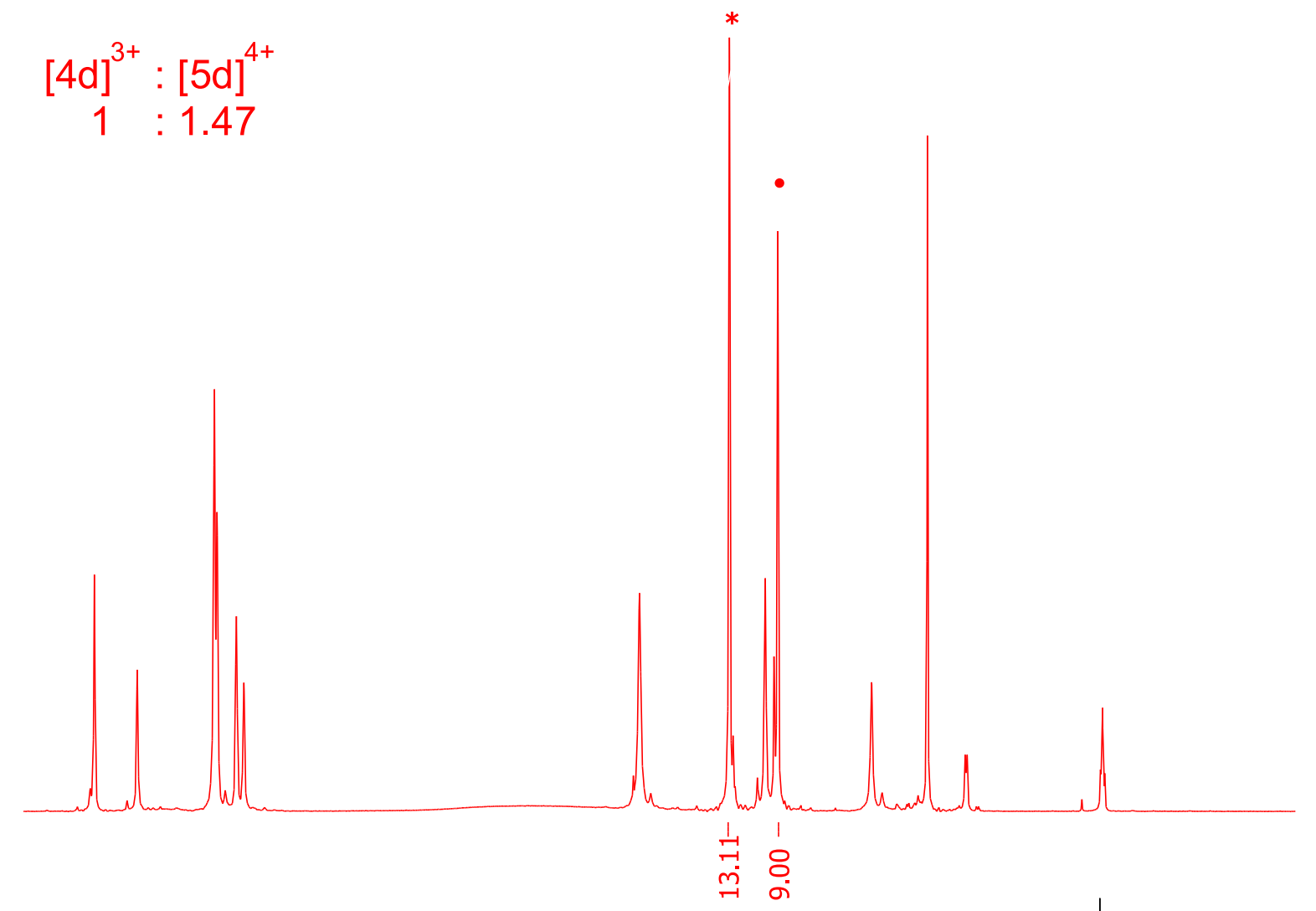

$$
\begin{gathered}
{[4 d]^{3+}:[5 d]^{4+}} \\
1: 0.10
\end{gathered}
$$

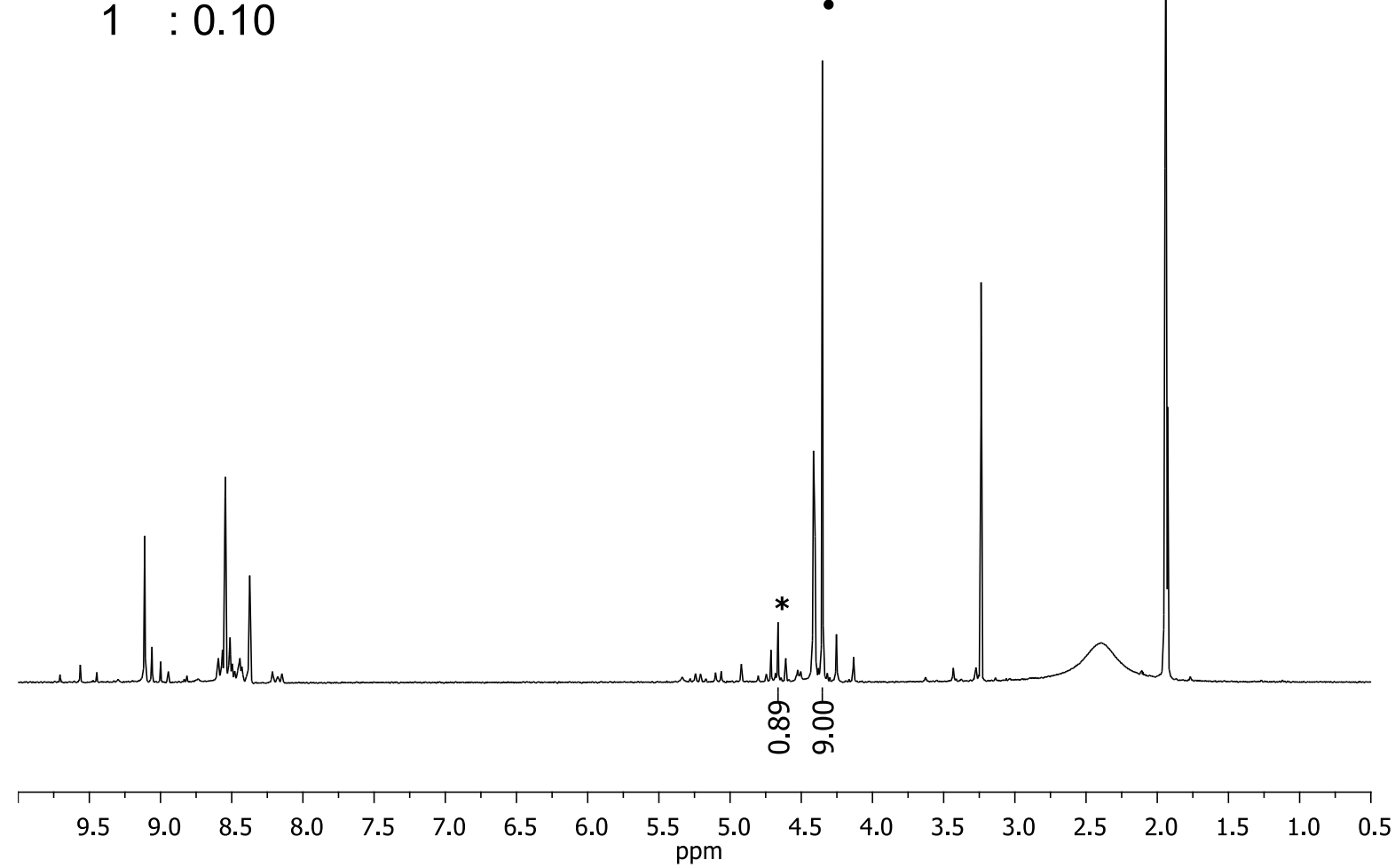

Figure S78. ${ }^{1} \mathrm{H}$ NMR spectra (recorded in $\mathrm{CD}_{3} \mathrm{CN}$ ) of the reaction mixtures obtained from reaction of 3d with $\mathrm{Me}_{3} \mathrm{OBF}_{4}$ in a $\mathrm{CH}_{2} \mathrm{Cl}_{2}$ solution (red) vs ball milling (black). Triazolium methyl substituents: [4d] $]^{3+}$ $=\bullet ;[5 \mathbf{d}]^{4+}=*$. 


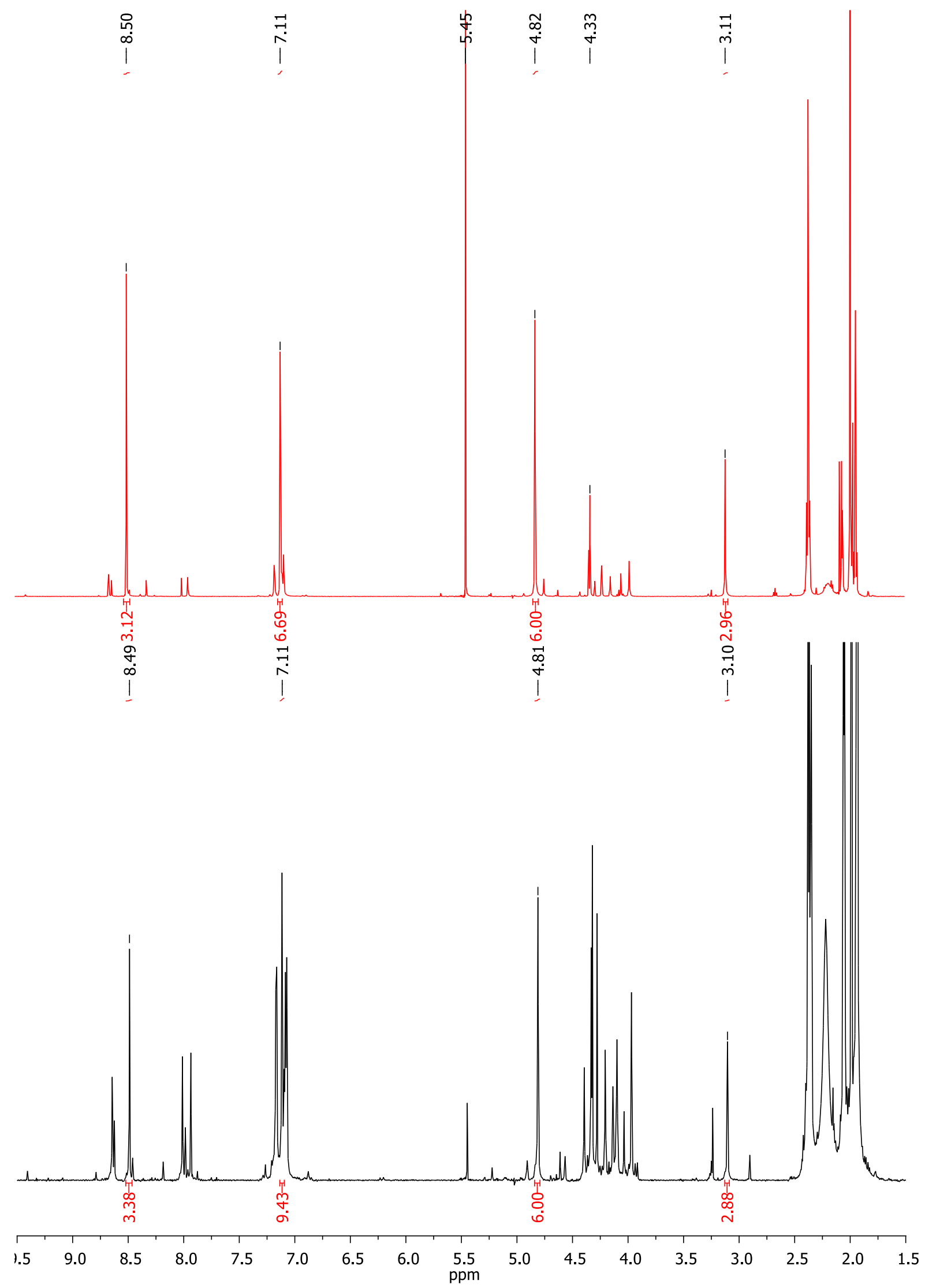

Figure S79. ${ }^{1} \mathrm{H}$ NMR spectra (recorded in $\mathrm{CD}_{3} \mathrm{CN}$ ) of the reaction mixtures obtained from reaction of 3a with 1.1 equivalent of $\mathrm{Me}_{3} \mathrm{OBF}_{4}$ in a $\mathrm{CH}_{2} \mathrm{Cl}_{2}$ solution (red) vs ball milling (black). [6a $]^{+}=*$. 


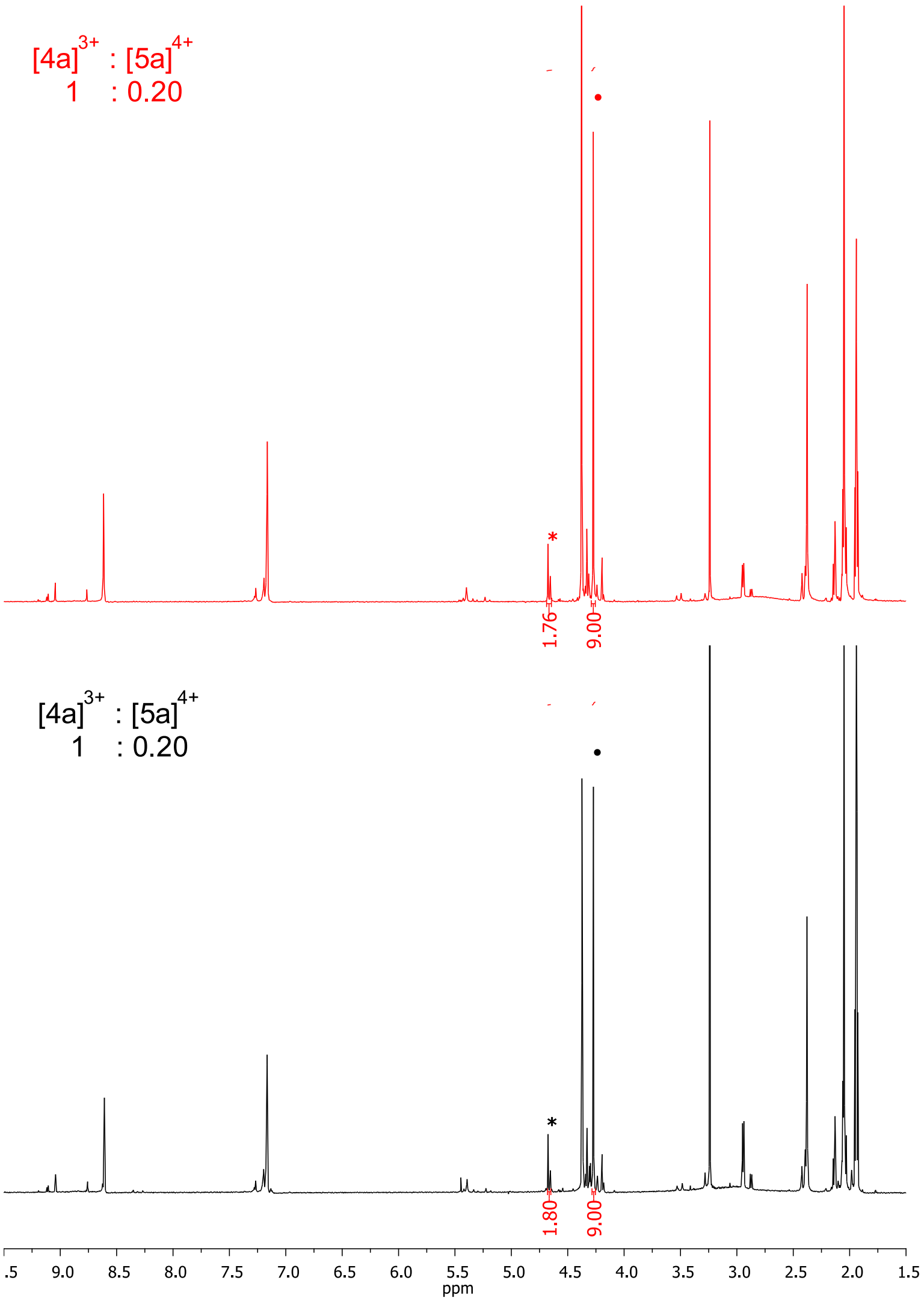

Figure S80. ${ }^{1} \mathrm{H}$ NMR spectra (recorded in $\mathrm{CD}_{3} \mathrm{CN}$ ) of the reaction mixtures obtained from ball milling 3a with: 4.5 equiv $\mathrm{Me}_{3} \mathrm{OBF}_{4}$ for a duration of: 1 hour(black); and 6 hour (red). Triazolium methyl substituents: $[\mathbf{4 a}]^{3+}=\bullet ;[\mathbf{5 a}]^{4+}=*$. 


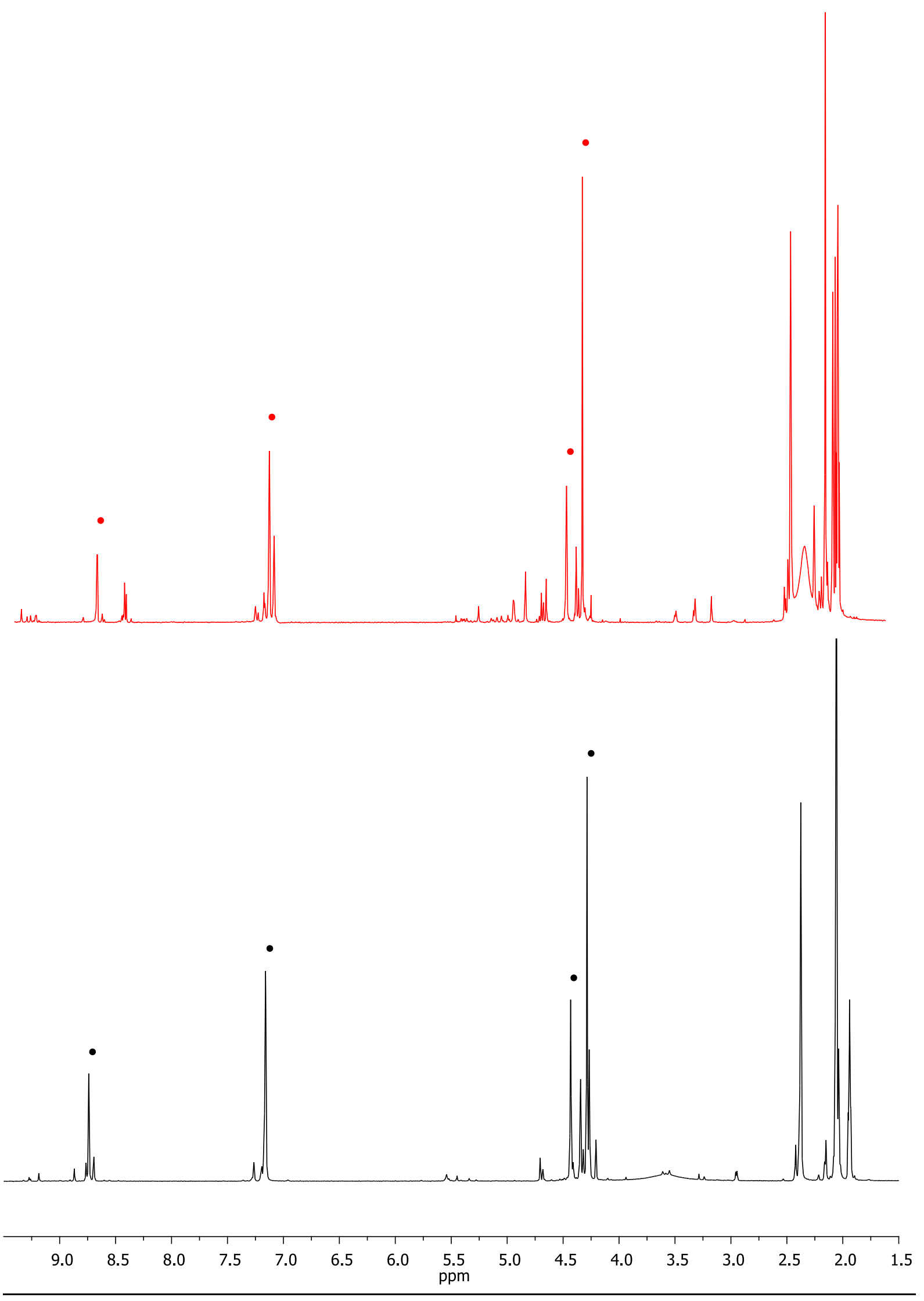

Figure S81. ${ }^{1} \mathrm{H}$ NMR spectra (recorded in $\mathrm{CD}_{3} \mathrm{CN}$ ) of the reaction mixtures obtained from reaction of 3a with MeOTf in a $\mathrm{CH}_{2} \mathrm{Cl}_{2}$ solution (red) vs ball milling (black). NMR signals for $[4 \mathbf{a}]^{3+}=\bullet$. 


$$
\begin{aligned}
{[4 b]^{3+} } & :[5 b]^{4+} \\
1 & : 0.42
\end{aligned}
$$
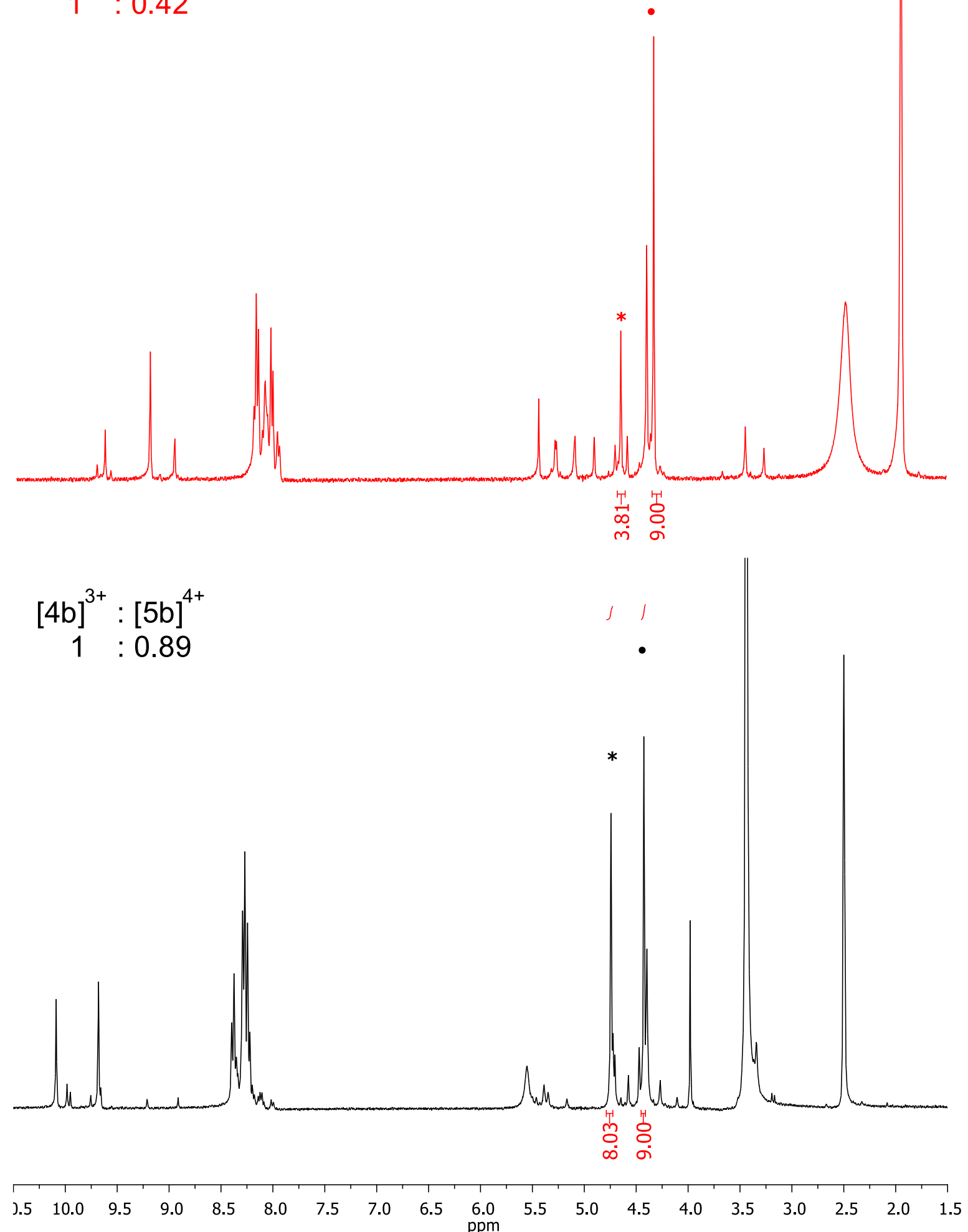

Figure S82. ${ }^{1} \mathrm{H}$ NMR spectra of the reaction mixtures obtained from reaction of $\mathbf{3 b}$ with MeOTf in a $\mathrm{CH}_{2} \mathrm{Cl}_{2}$ solution (red) vs ball milling (black). Triazolium methyl substituents: $[\mathbf{4 b}]^{3+}=\boldsymbol{\bullet} ;[\mathbf{5 b}]^{4+}=*$. These spectra were recorded in DMSO- $\mathrm{d}_{6}$ and $\mathrm{CD}_{3} \mathrm{CN}$, respectively. 


$$
\begin{aligned}
& {[4 c]^{3+}:[5 c]^{4+}:[6 c]^{+} } \\
& 1: 0.10: 1.56
\end{aligned}
$$

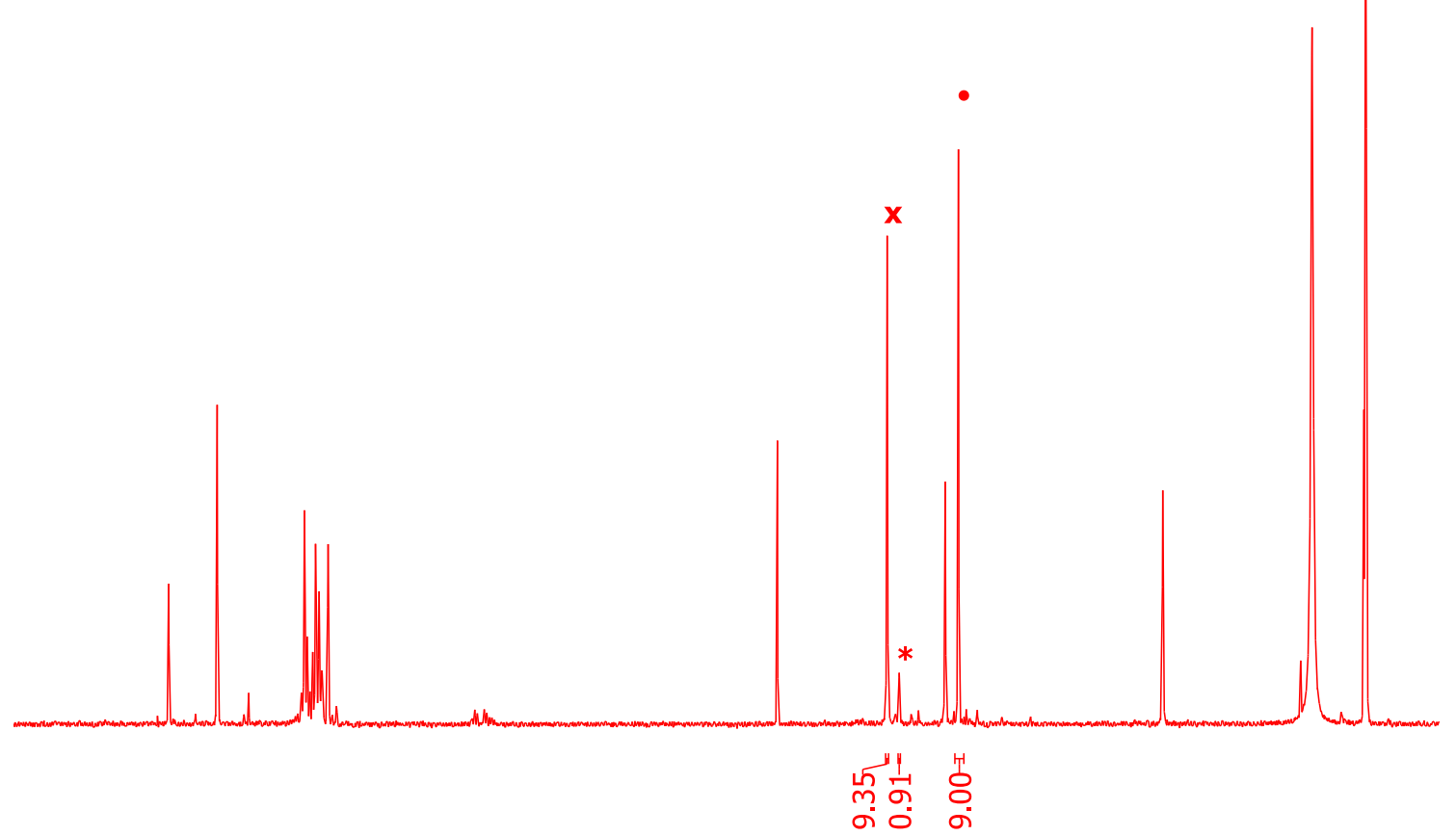

$$
[4 c]^{3+}:[5 c]^{4+}:[6 c]^{+}
$$

$1: 0.34: 0.31$

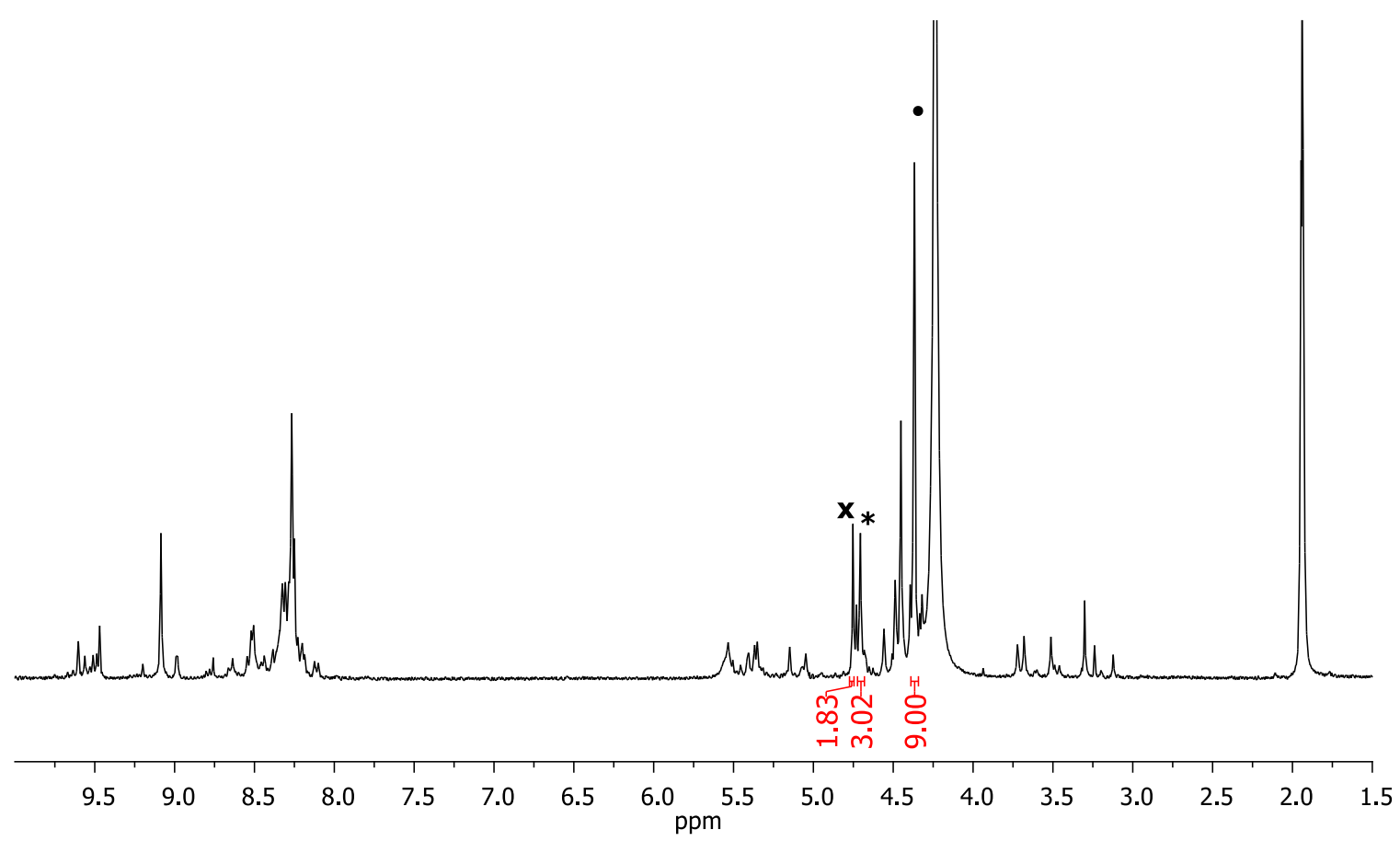

Figure S83. ${ }^{1} \mathrm{H}$ NMR spectra (recorded in $\mathrm{CD}_{3} \mathrm{CN}$ ) of the reaction mixtures obtained from reaction of $3 \mathrm{c}$ with MeOTf in a $\mathrm{CH}_{2} \mathrm{Cl}_{2}$ solution (red) vs ball milling (black). Triazolium methyl substituents: $[4 \mathrm{c}]^{3+}=$ $\bullet ;[5]^{4+}=*$. Methylene groups in $[6 \mathbf{c}]^{+}=\mathbf{x}$. 


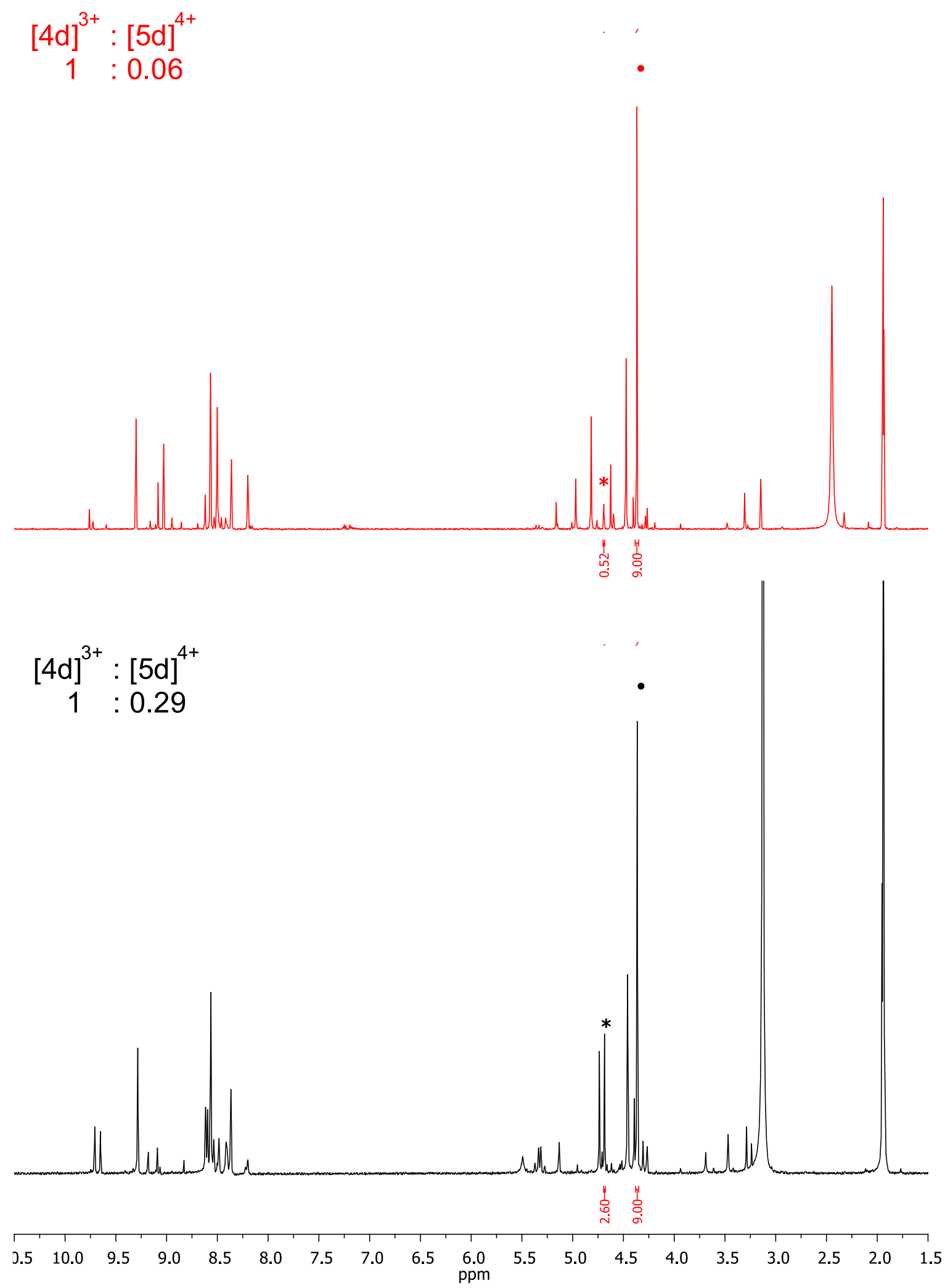

Figure S84. ${ }^{1} \mathrm{H}$ NMR spectra (recorded in $\mathrm{CD}_{3} \mathrm{CN}$ ) of the reaction mixtures obtained from reaction of 3d with MeOTf in a $\mathrm{CH}_{2} \mathrm{Cl}_{2}$ solution (red) vs ball milling (black). Triazolium methyl substituents: [4d $]^{3+}$ $=\bullet ;[5 d]^{4+}=*$. 


\section{Mass Spectrometry}
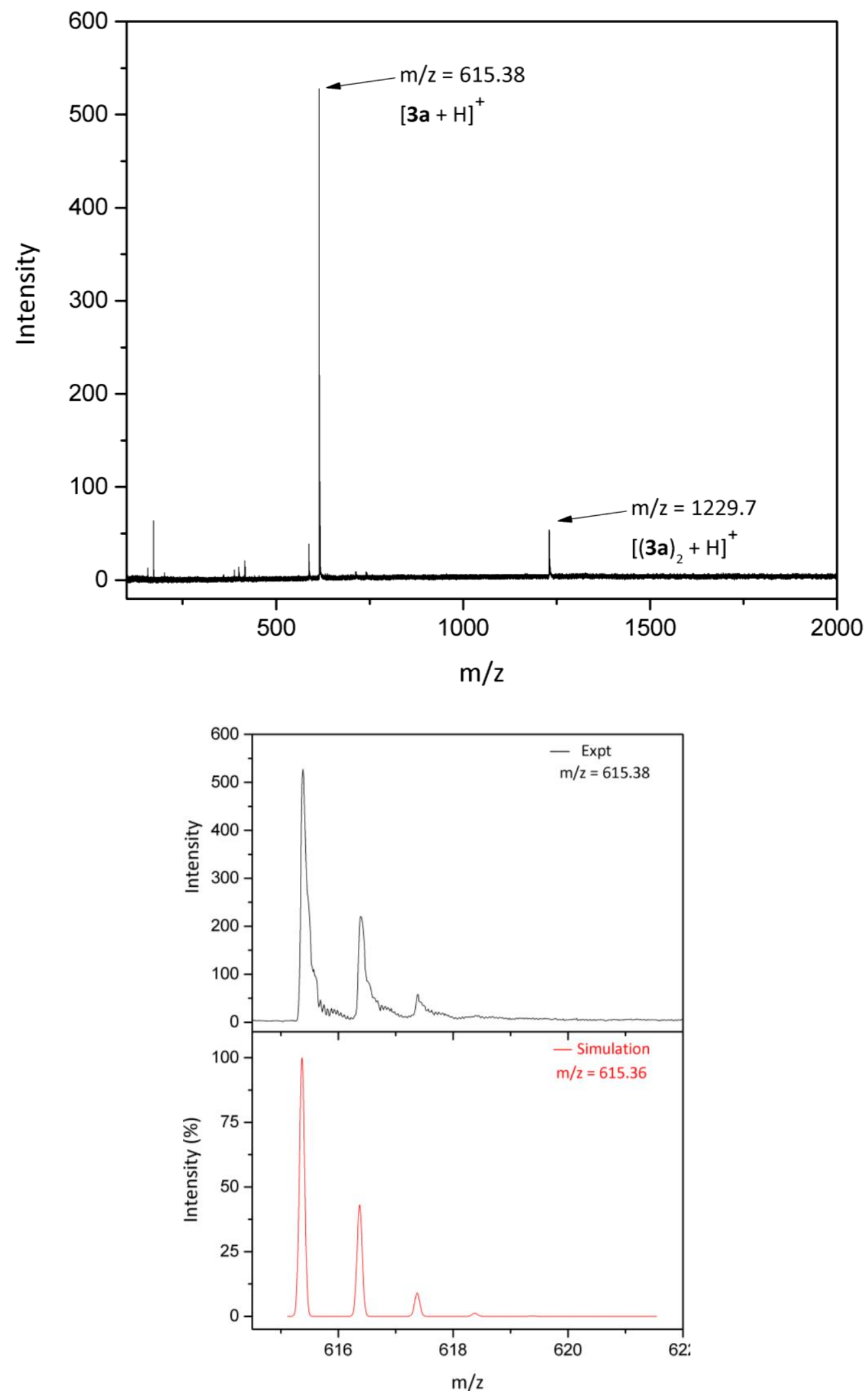

Figure S85. ESI mass spectrum of 3a, recorded in a mixture of $\mathrm{CHCl}_{3}$ and $\mathrm{MeOH}$ using positive ion mode, plus an expansion and isotope simulation of the parent ion. 

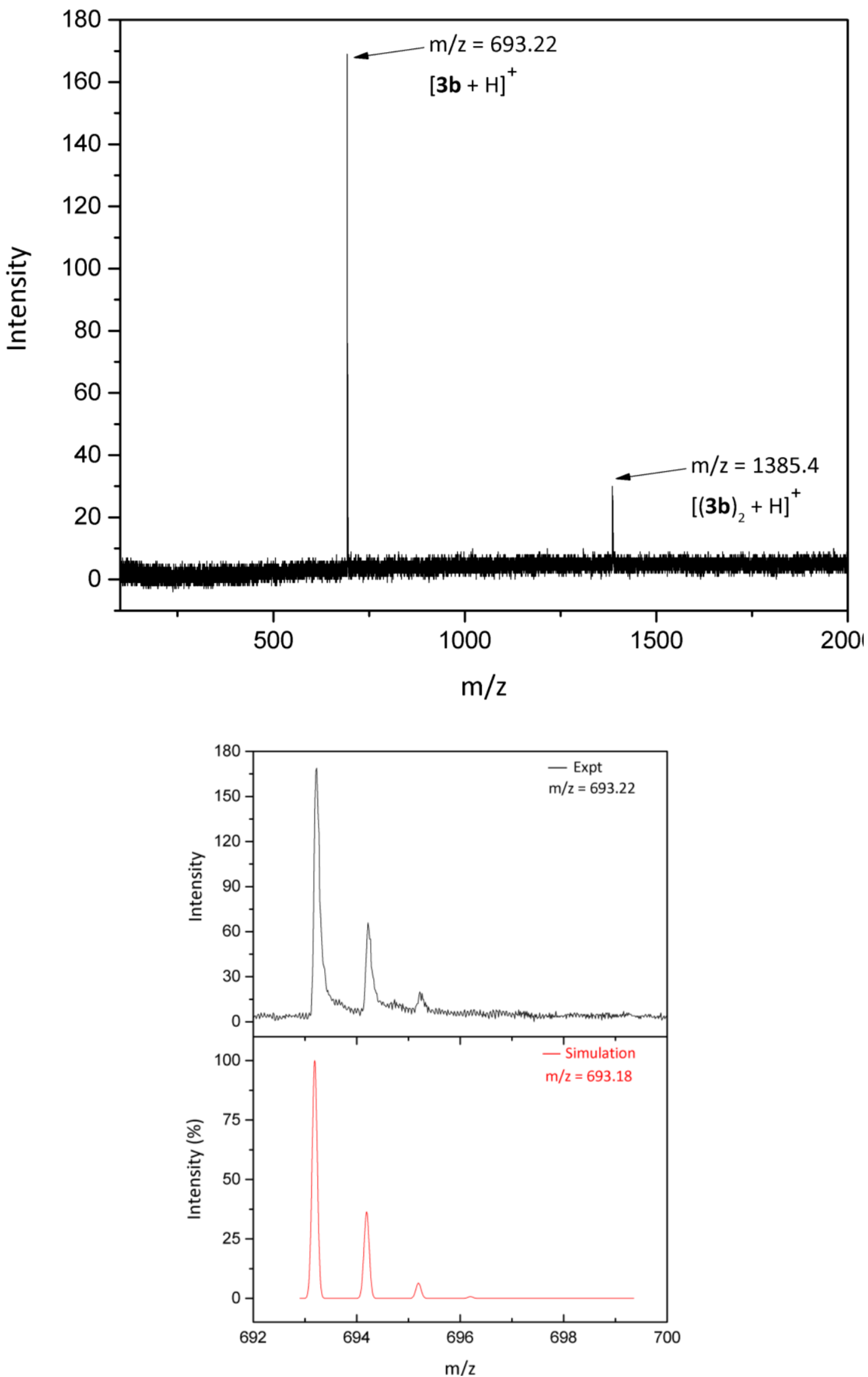

Figure S86. ESI mass spectrum of $\mathbf{3 b}$, recorded in a mixture of $\mathrm{CHCl}_{3}$ and $\mathrm{MeOH}$ using positive ion mode, plus an expansion and isotope simulation of the parent ion. 

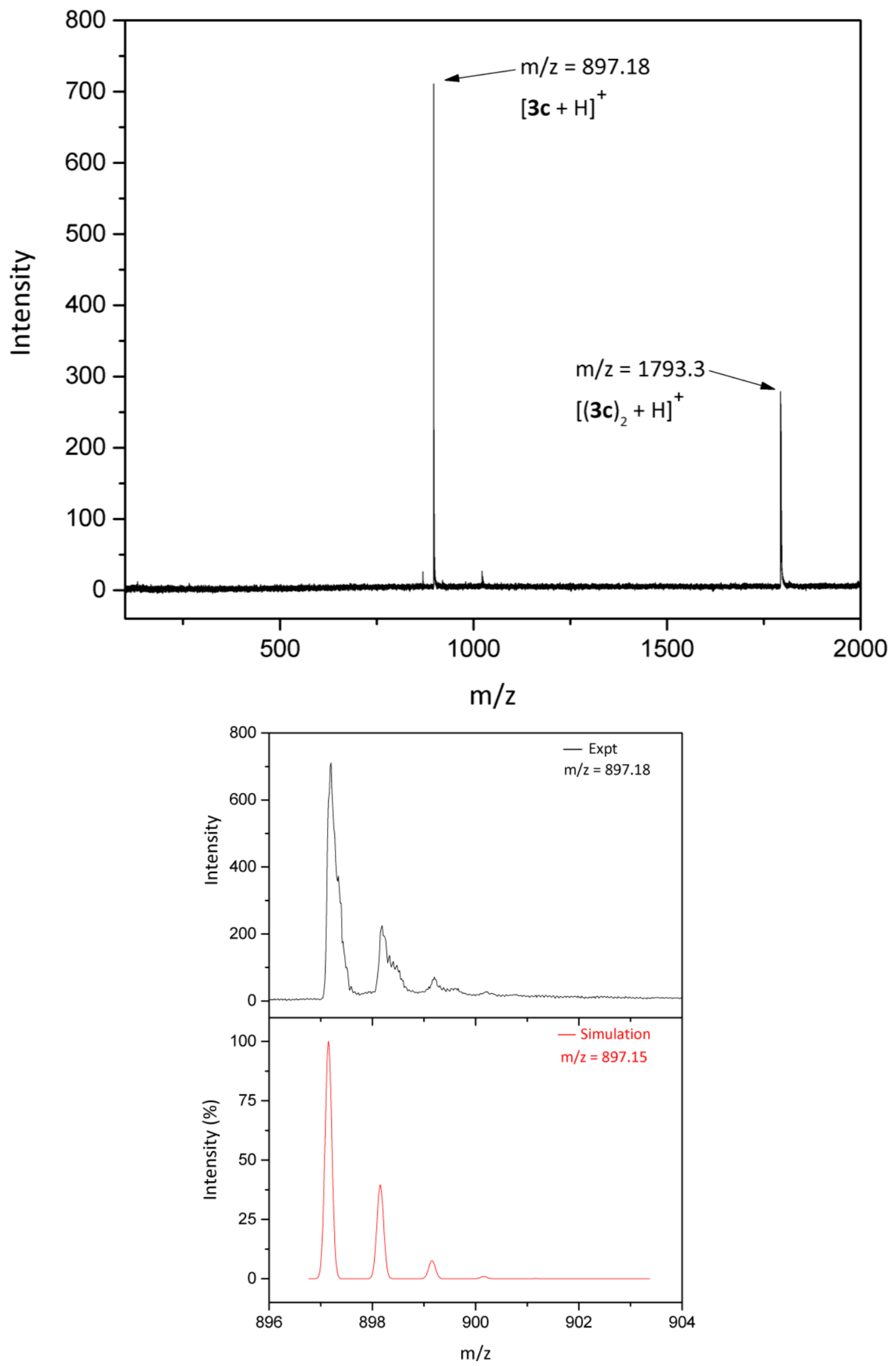

Figure S87. ESI mass spectrum of 3c, recorded in a mixture of $\mathrm{CHCl}_{3}$ and $\mathrm{MeOH}$ using positive ion mode, plus an expansion and isotope simulation of the parent ion. 

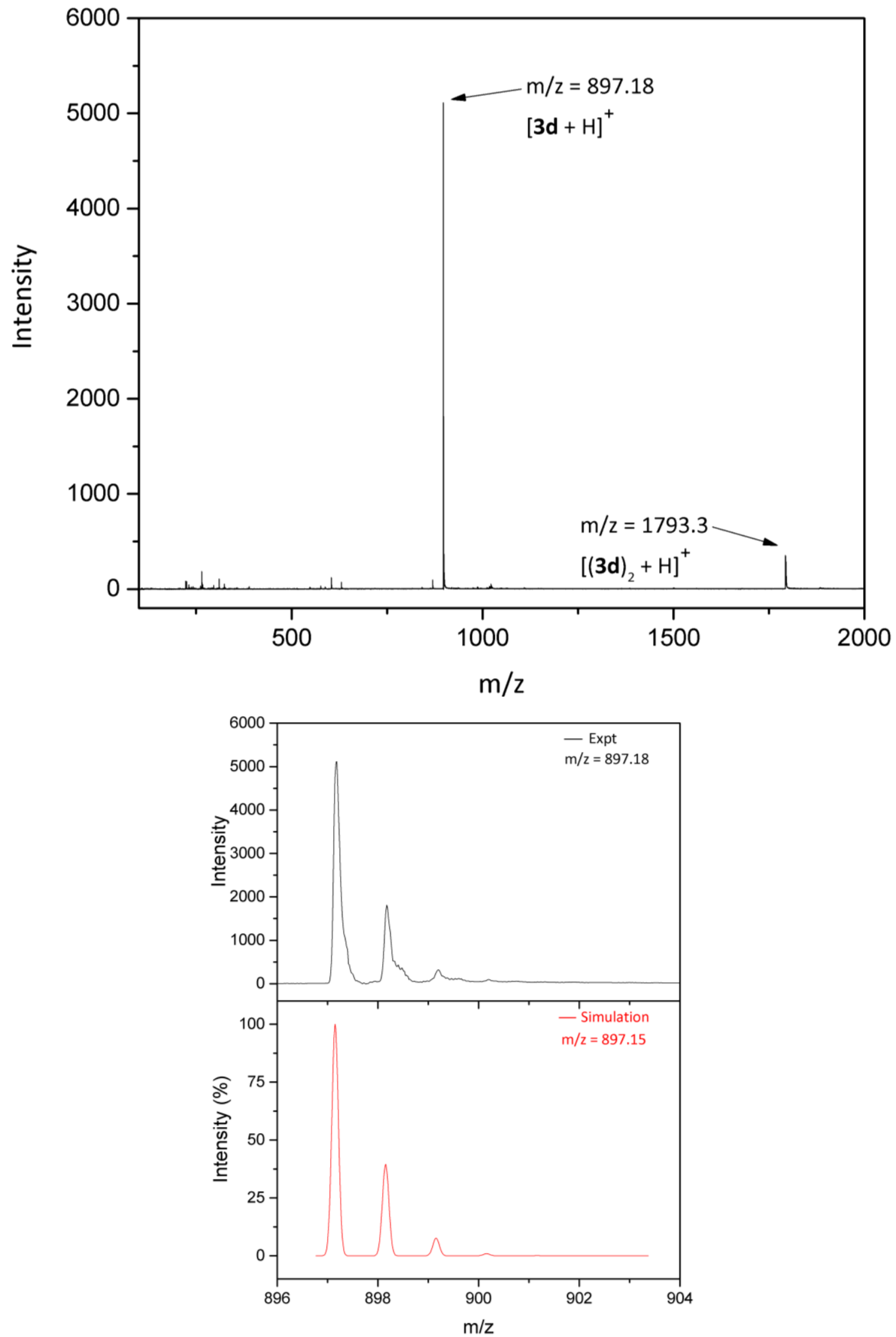

Figure S88. ESI mass spectrum of 3d, recorded in a mixture of $\mathrm{CHCl}_{3}$ and $\mathrm{MeOH}$ using positive ion mode, plus an expansion and isotope simulation of the parent ion. 

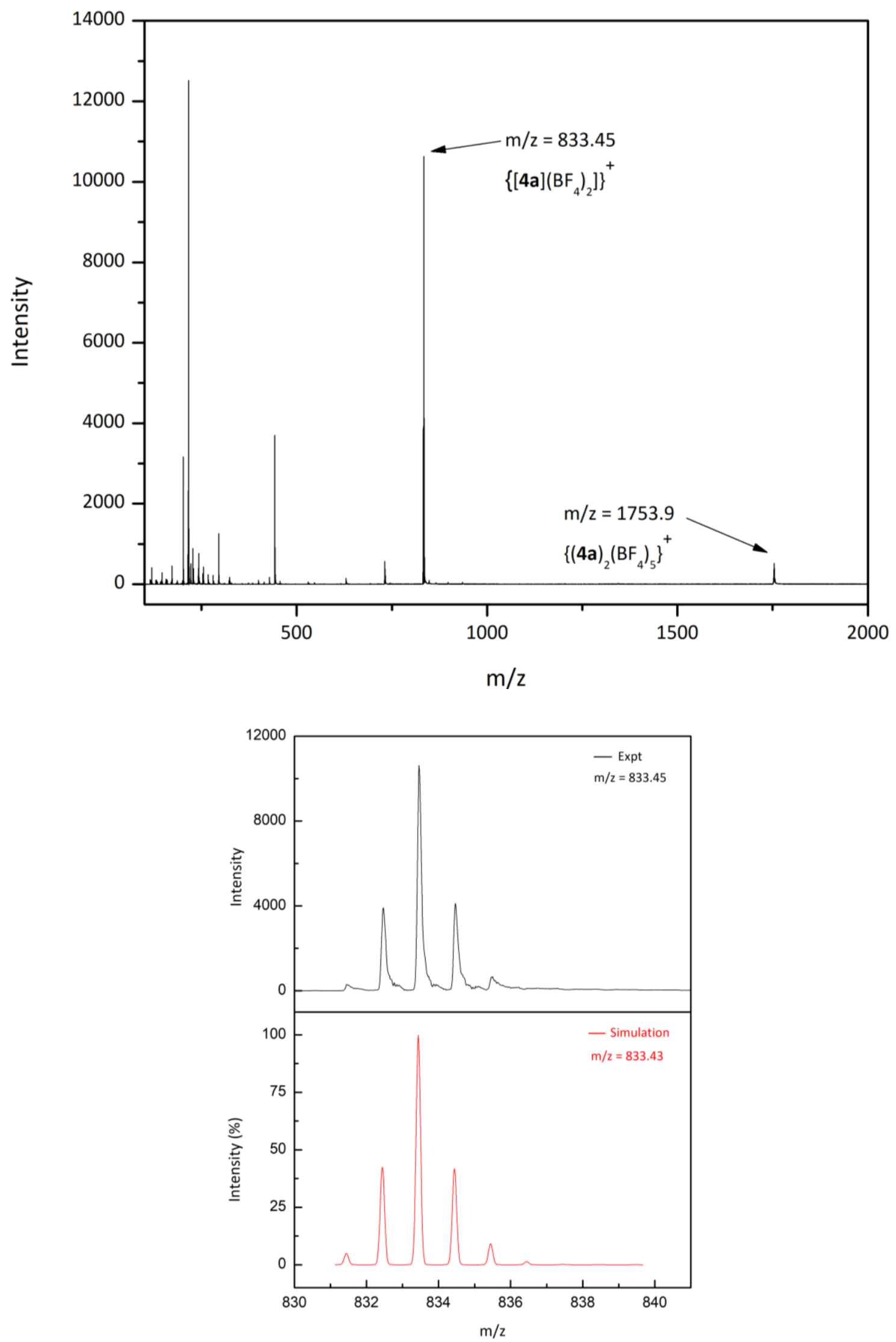

Figure S89. ESI mass spectrum of $[4 a]\left(\mathrm{BF}_{4}\right)_{3}$, recorded in $\mathrm{CH}_{3} \mathrm{CN}$ solution using positive ion mode, plus an expansion and isotope simulation of the parent ion. 

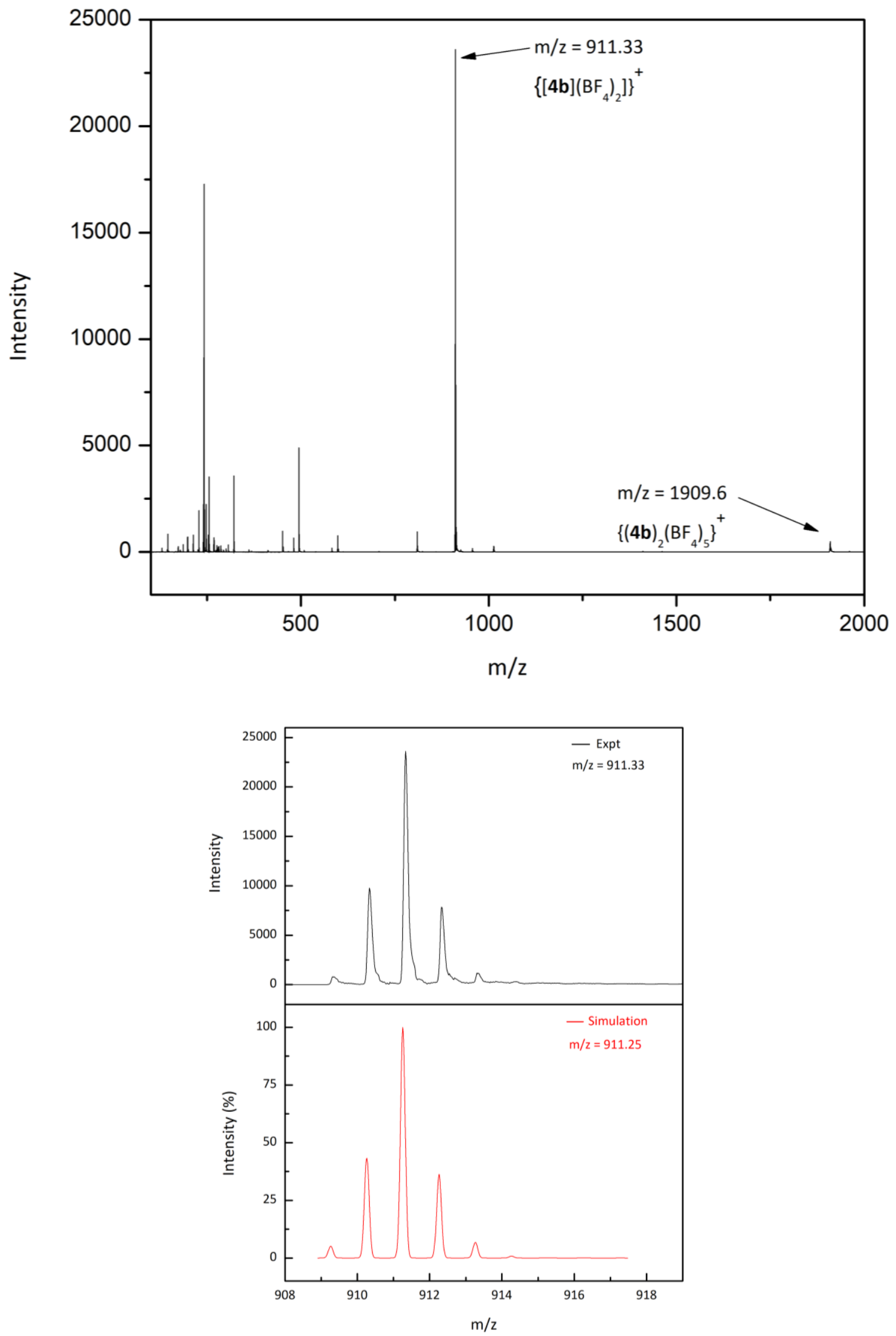

Figure 590. ESI mass spectrum of $[4 \mathbf{b}]\left(\mathrm{BF}_{4}\right)_{3}$, recorded in $\mathrm{CH}_{3} \mathrm{CN}$ solution using positive ion mode, plus an expansion and isotope simulation of the parent ion. 

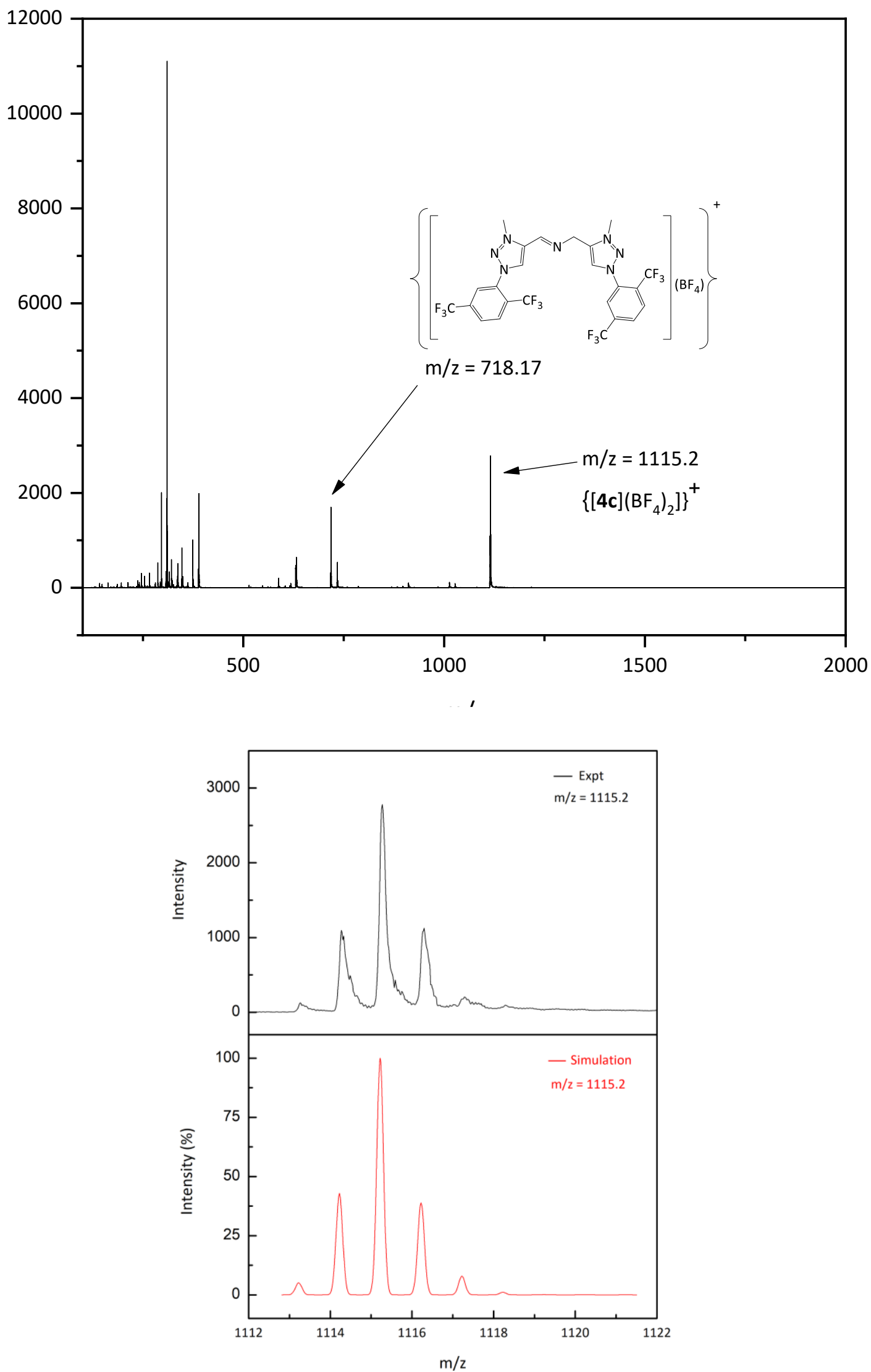

Figure S91. ESI mass spectrum of $[4 \mathrm{c}]\left(\mathrm{BF}_{4}\right)_{3}$, recorded in $\mathrm{CH}_{3} \mathrm{CN}$ solution using positive ion mode, plus an expansion and isotope simulation of the parent ion. 

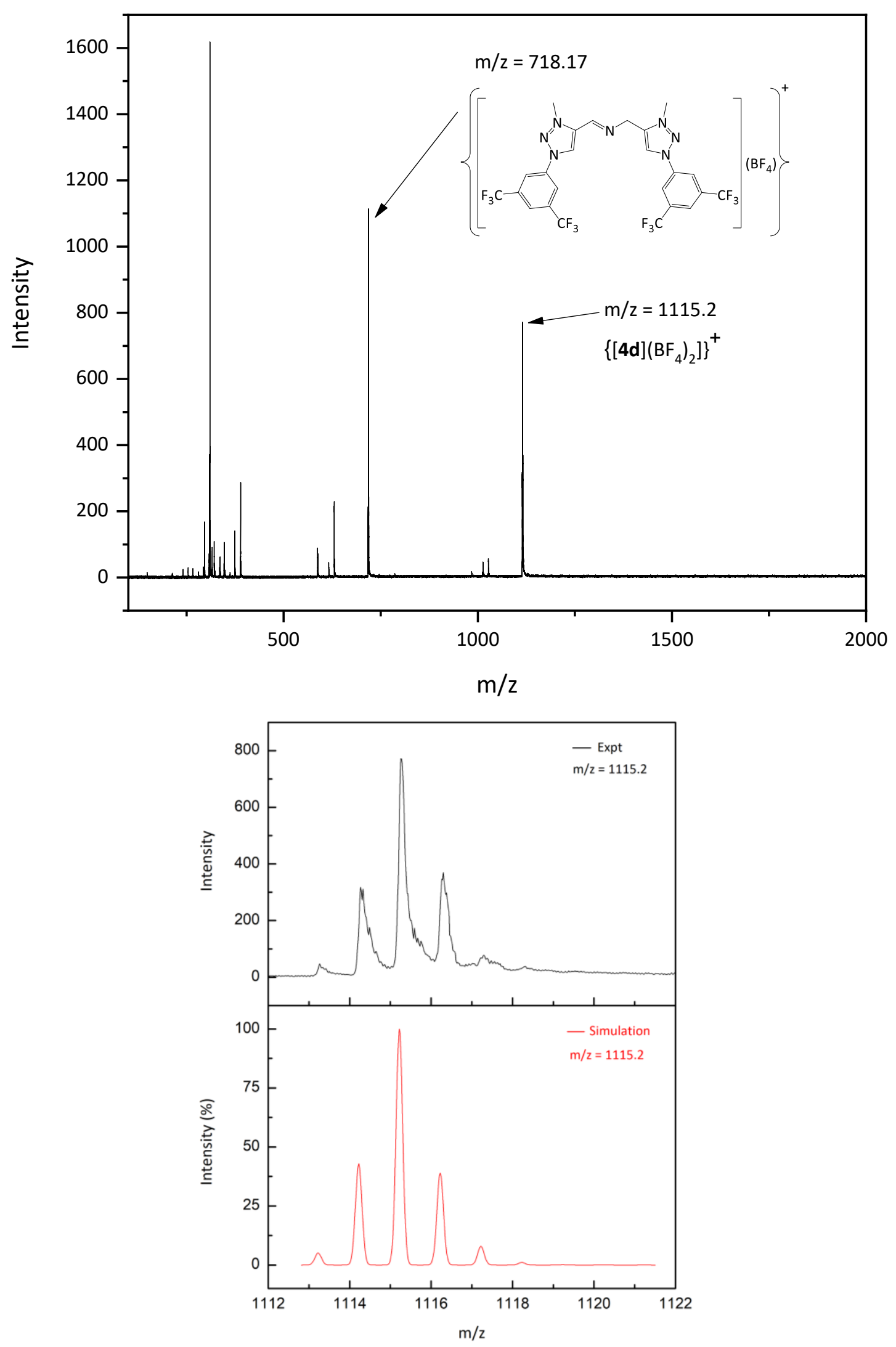

Figure S92. ESI mass spectrum of [4d] $\left(\mathrm{BF}_{4}\right)_{3}$, recorded in $\mathrm{CH}_{3} \mathrm{CN}$ solution using positive ion mode, plus an expansion and isotope simulation of the parent ion. 

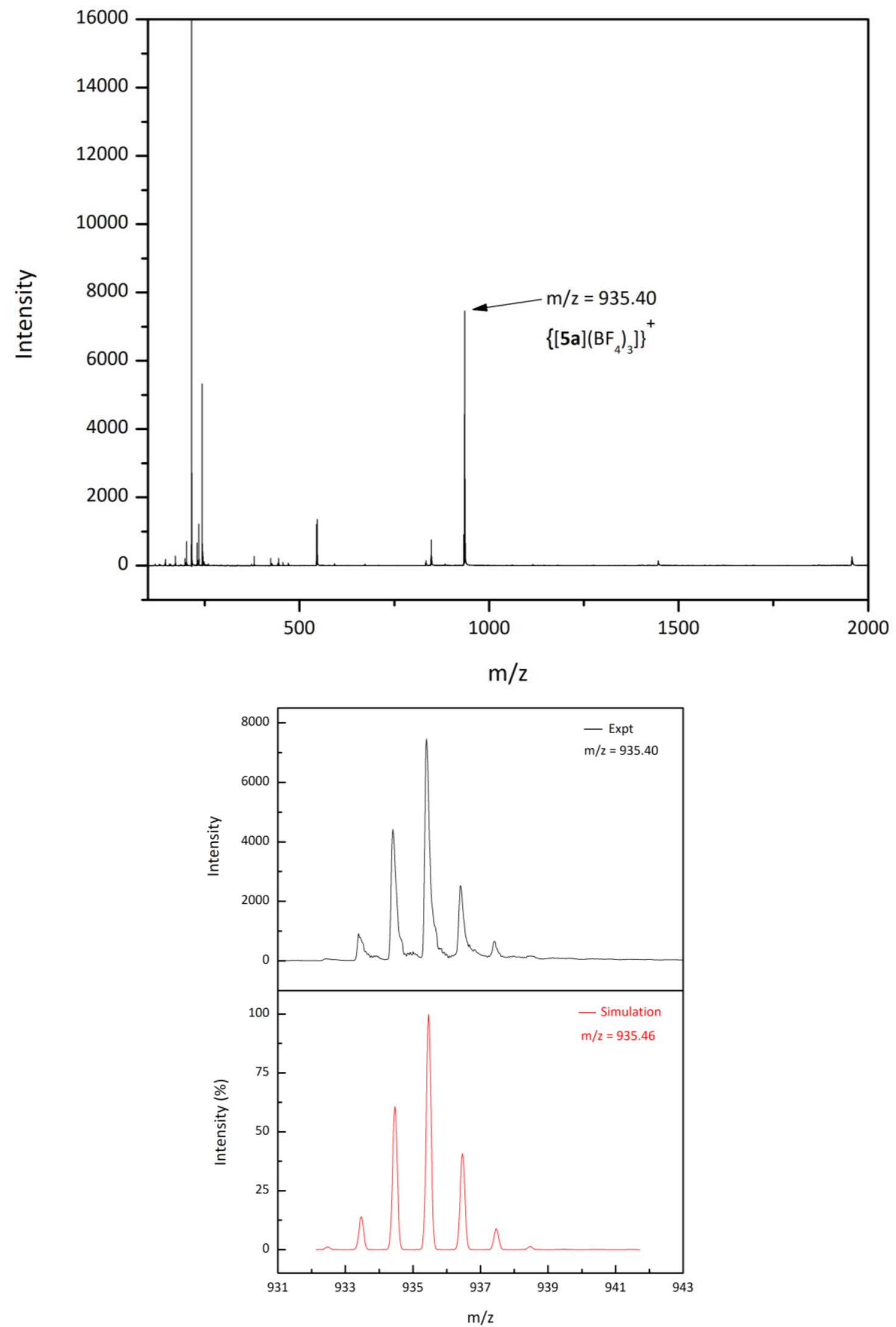

Figure S93. ESI mass spectrum of [5a](BF $\mathrm{BF}_{4}$, recorded in $\mathrm{CH}_{3} \mathrm{CN}$ solution using positive ion mode, plus an expansion and isotope simulation of the parent ion. 

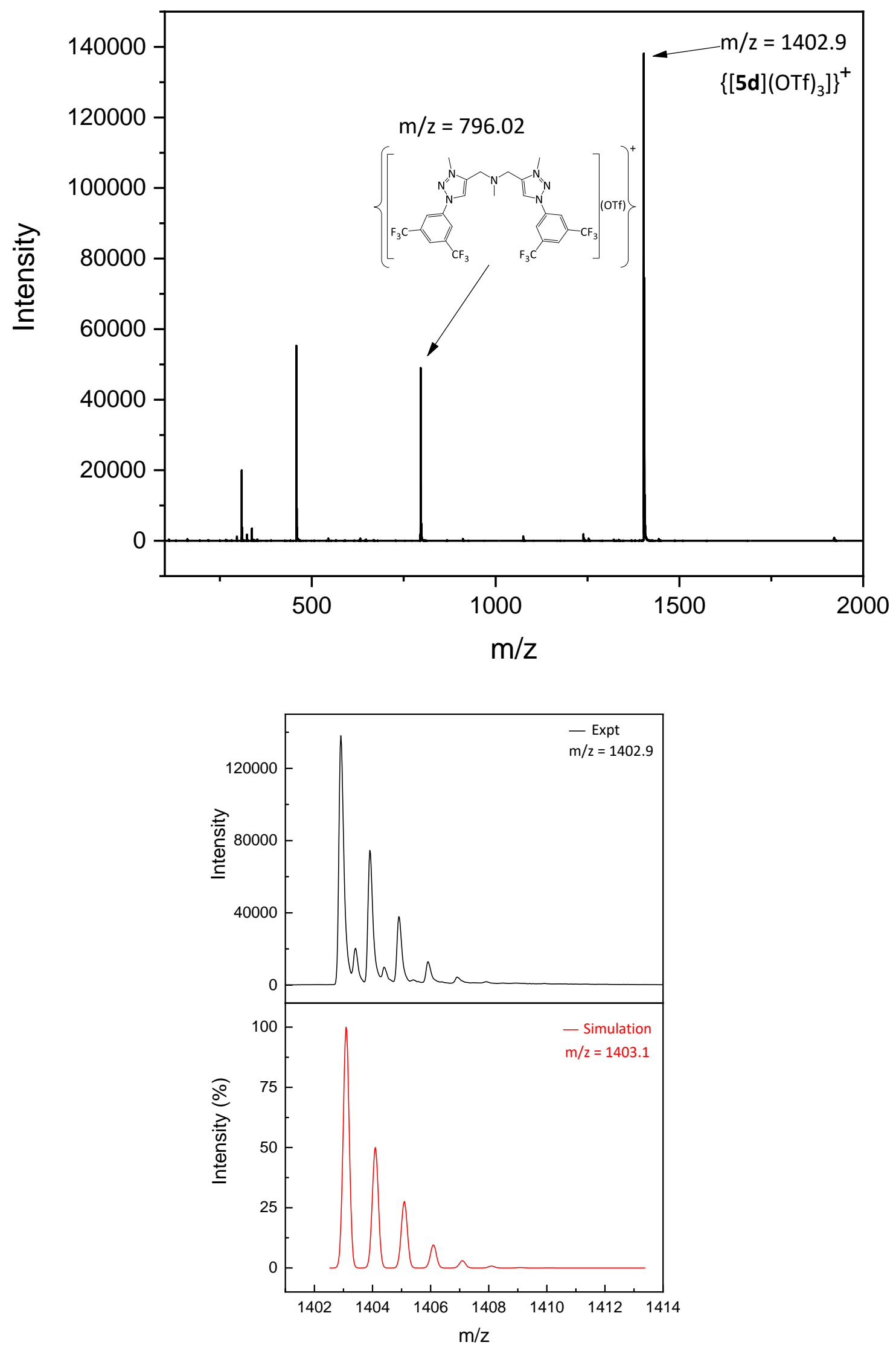

Figure S94. ESI mass spectrum of [5d](OTf $)_{4}$, recorded in $\mathrm{CH}_{3} \mathrm{CN}$ solution using positive ion mode, plus an expansion and isotope simulation of the parent ion. 

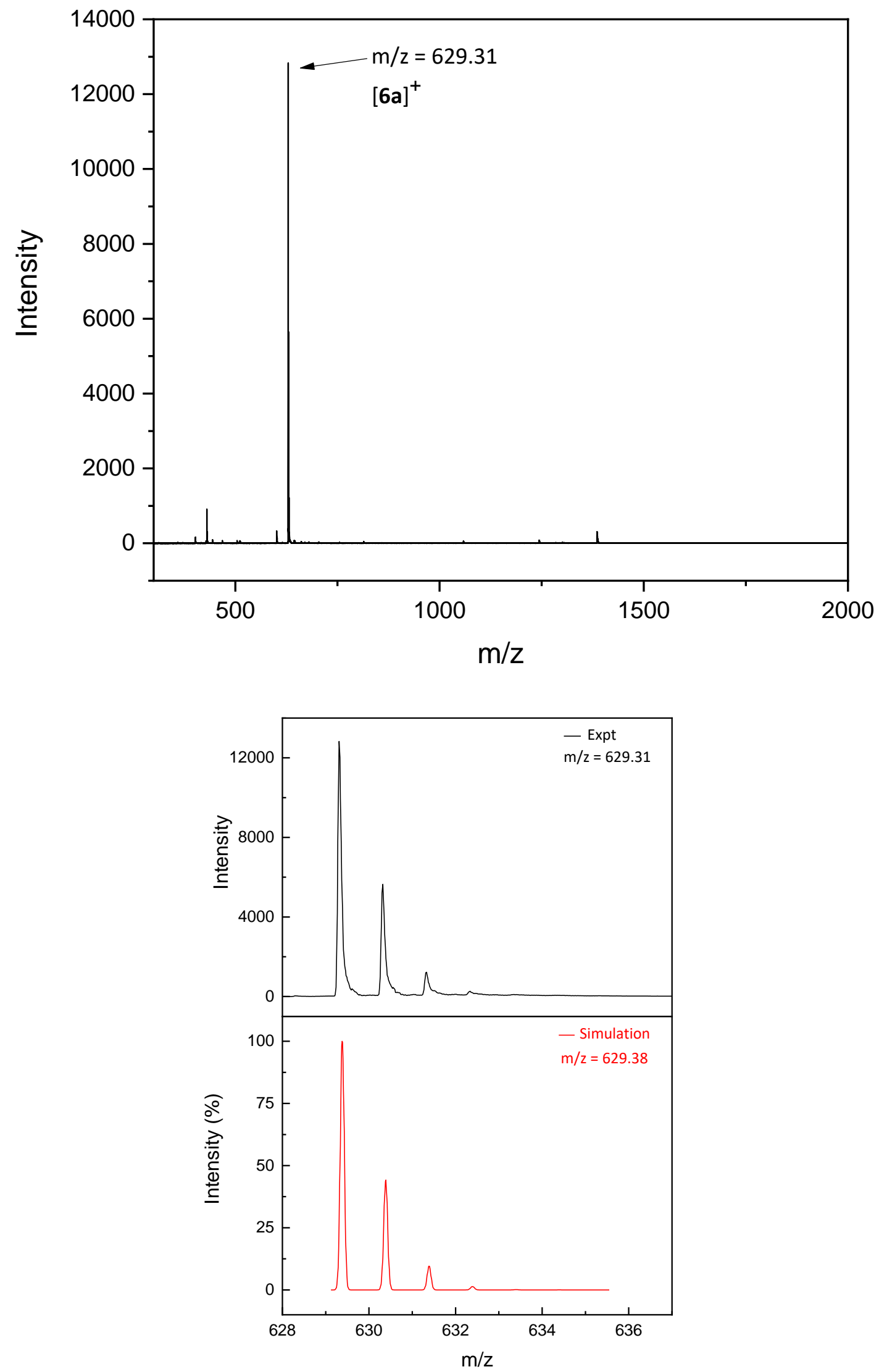

Figure S95. ESI mass spectrum of [6a](I), recorded in $\mathrm{CH}_{3} \mathrm{CN}$ solution using positive ion mode, plus an expansion and isotope simulation of the parent ion. 

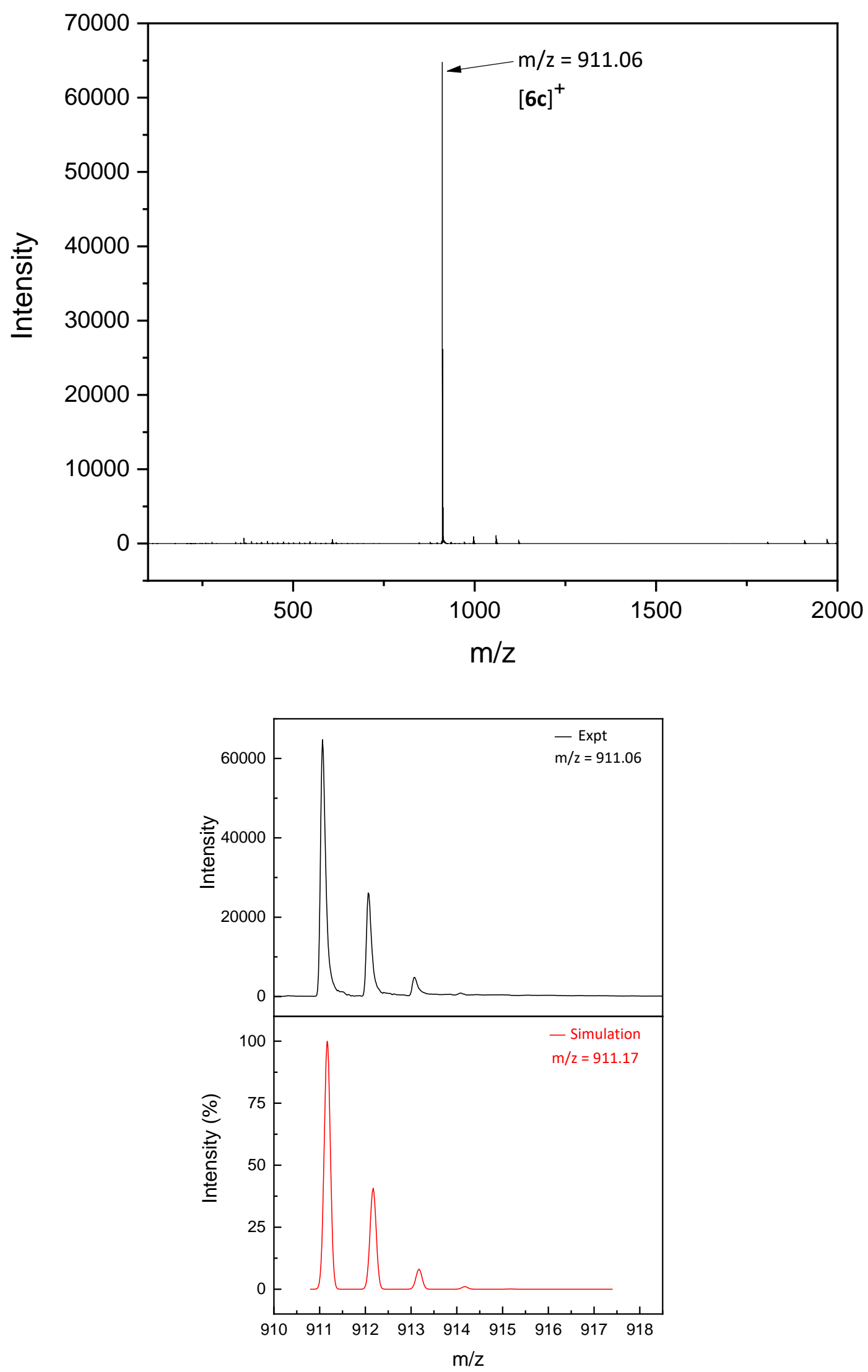

Figure S96. ESI mass spectrum of [6c](OTf), recorded in $\mathrm{CH}_{3} \mathrm{CN}$ solution using positive ion mode, plus an expansion and isotope simulation of the parent ion. 

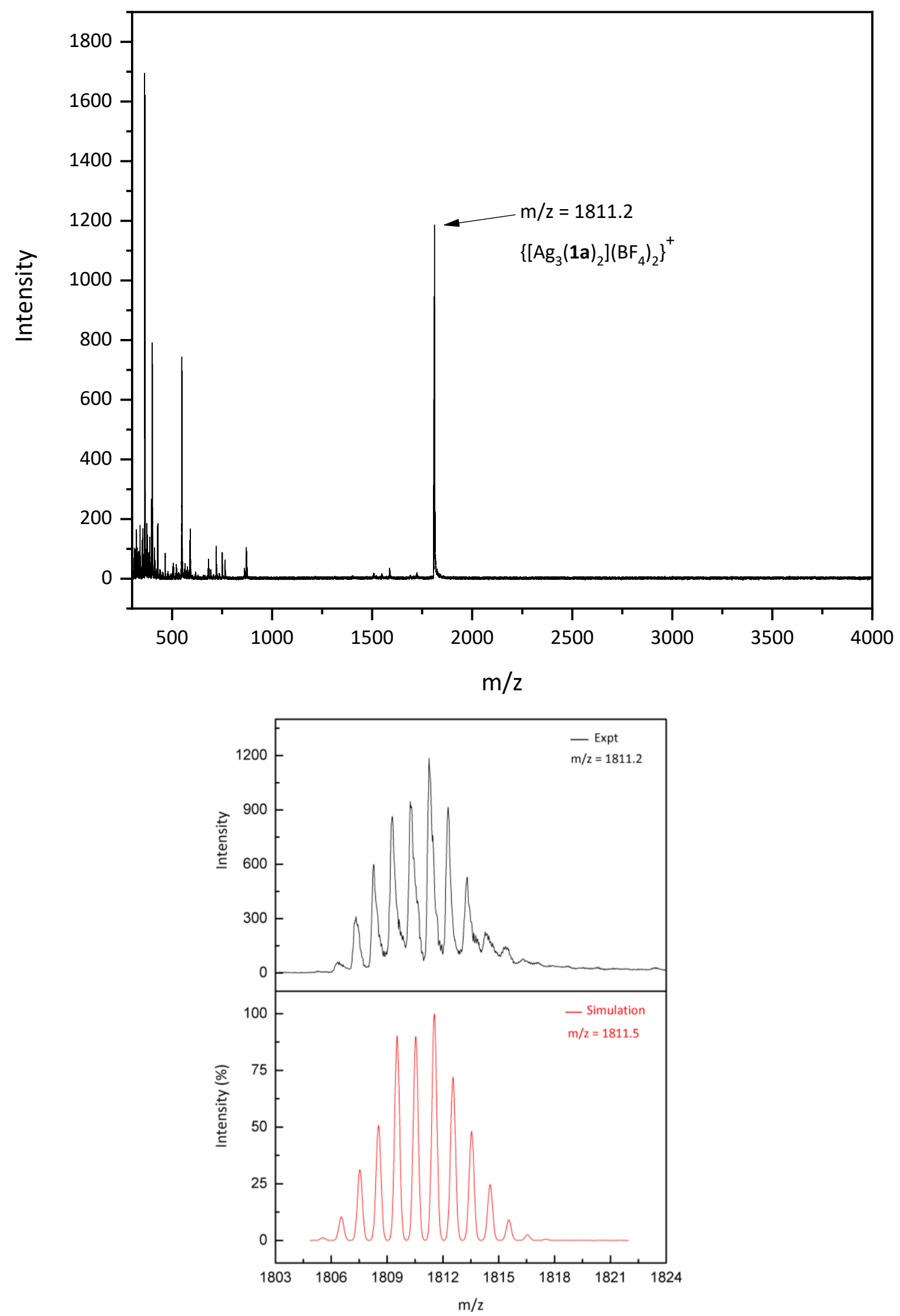

Figure S97. ESI mass spectrum of $\left[\mathrm{Ag}_{3}(1 \mathrm{a})_{2}\right]\left[\mathrm{BF}_{4}\right]_{3}$, recorded in $\mathrm{CH}_{3} \mathrm{CN}$ solution using positive ion mode, plus an expansion and isotope simulation of the parent ion. 

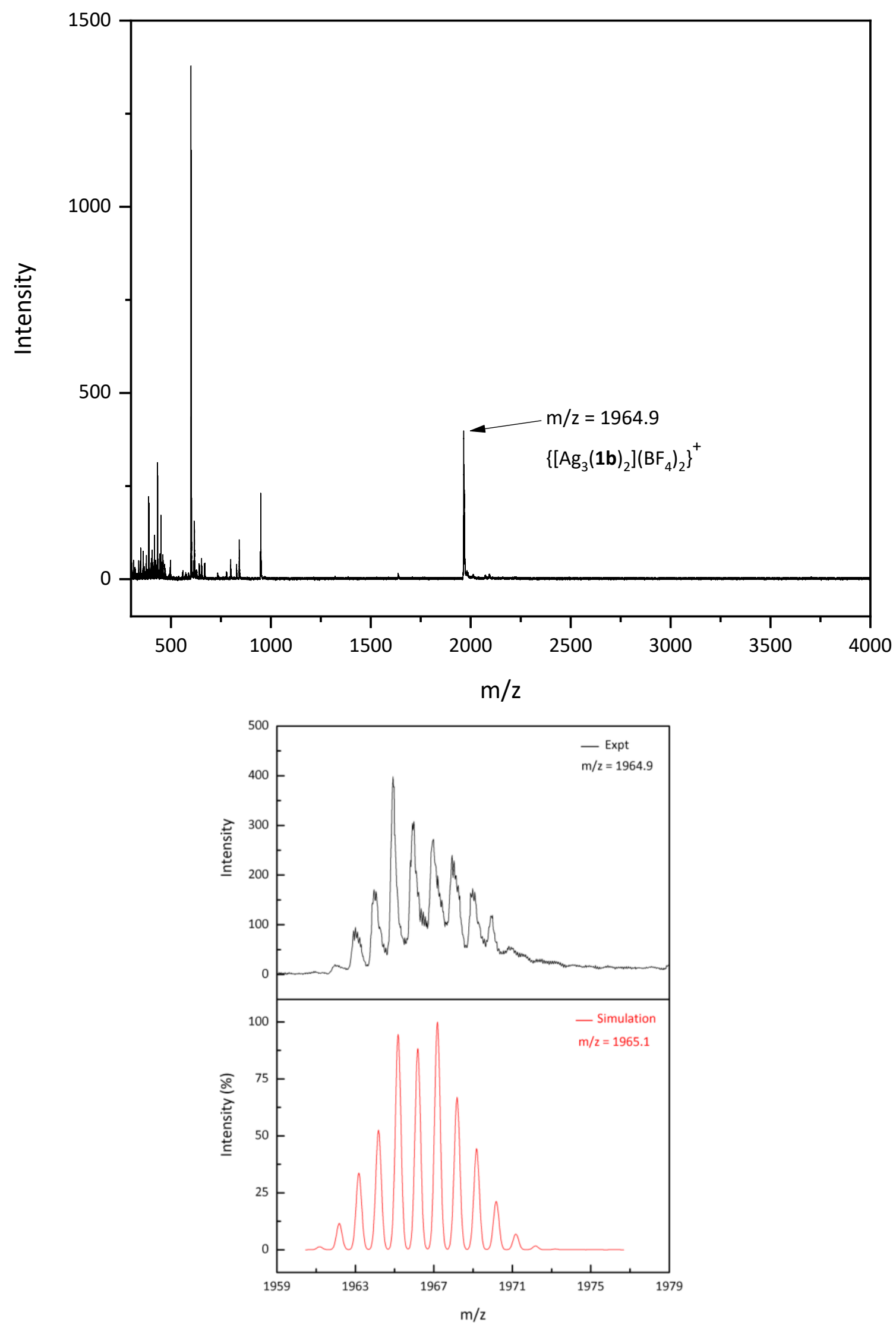

Figure S98. ESI mass spectrum of $\left[\mathrm{Ag}_{3}(\mathbf{1} \mathbf{b})_{2}\right]\left[\mathrm{BF}_{4}\right]_{3}$, recorded in $\mathrm{CH}_{3} \mathrm{CN}$ solution using positive ion mode, plus an expansion and isotope simulation of the parent ion. 

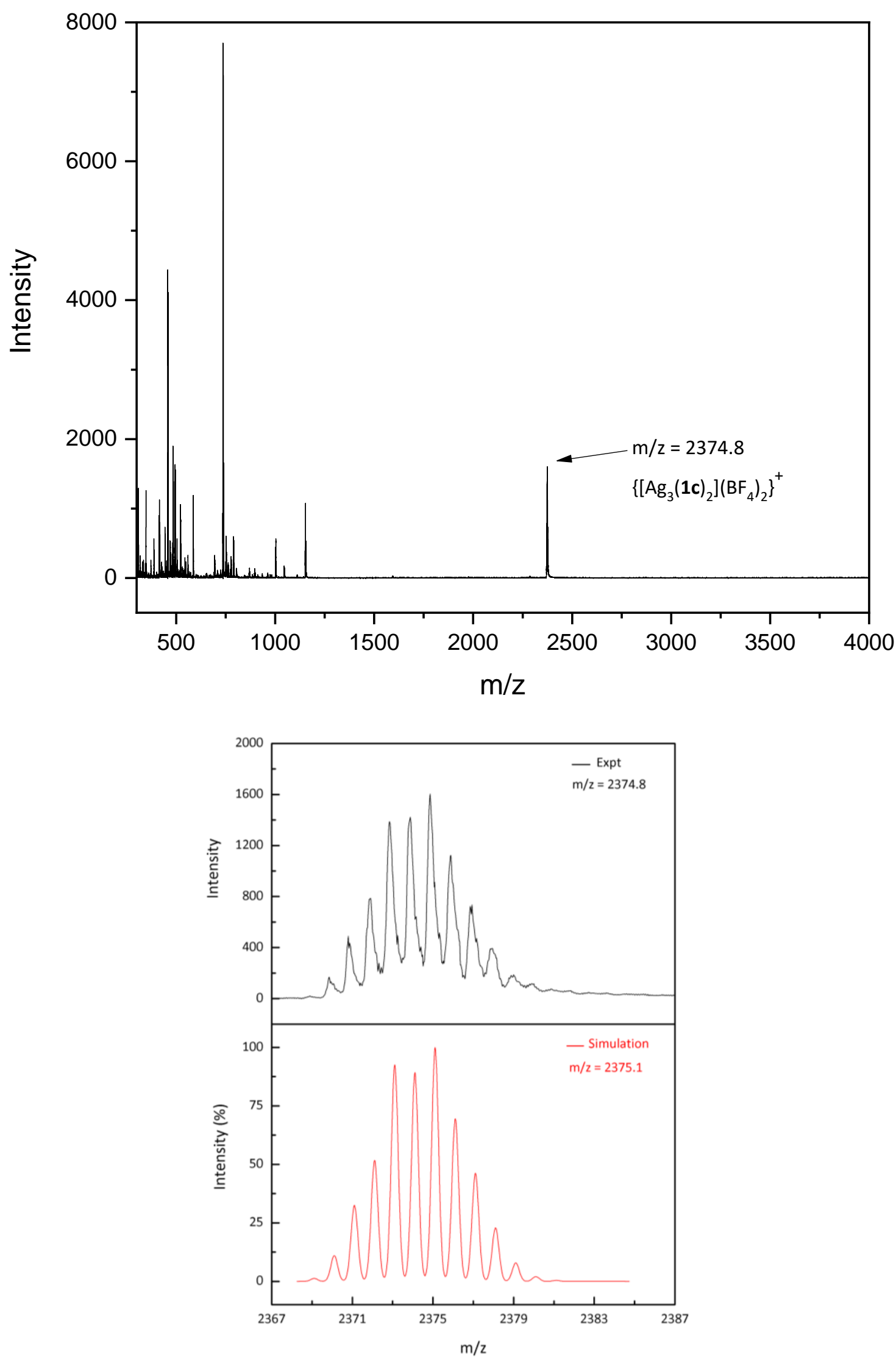

Figure S99. ESI mass spectrum of $\left[\mathrm{Ag}_{3}(1 \mathrm{c})_{2}\right]\left[\mathrm{BF}_{4}\right]_{3}$, recorded in $\mathrm{CH}_{3} \mathrm{CN}$ solution using positive ion mode, plus an expansion and isotope simulation of the parent ion. 

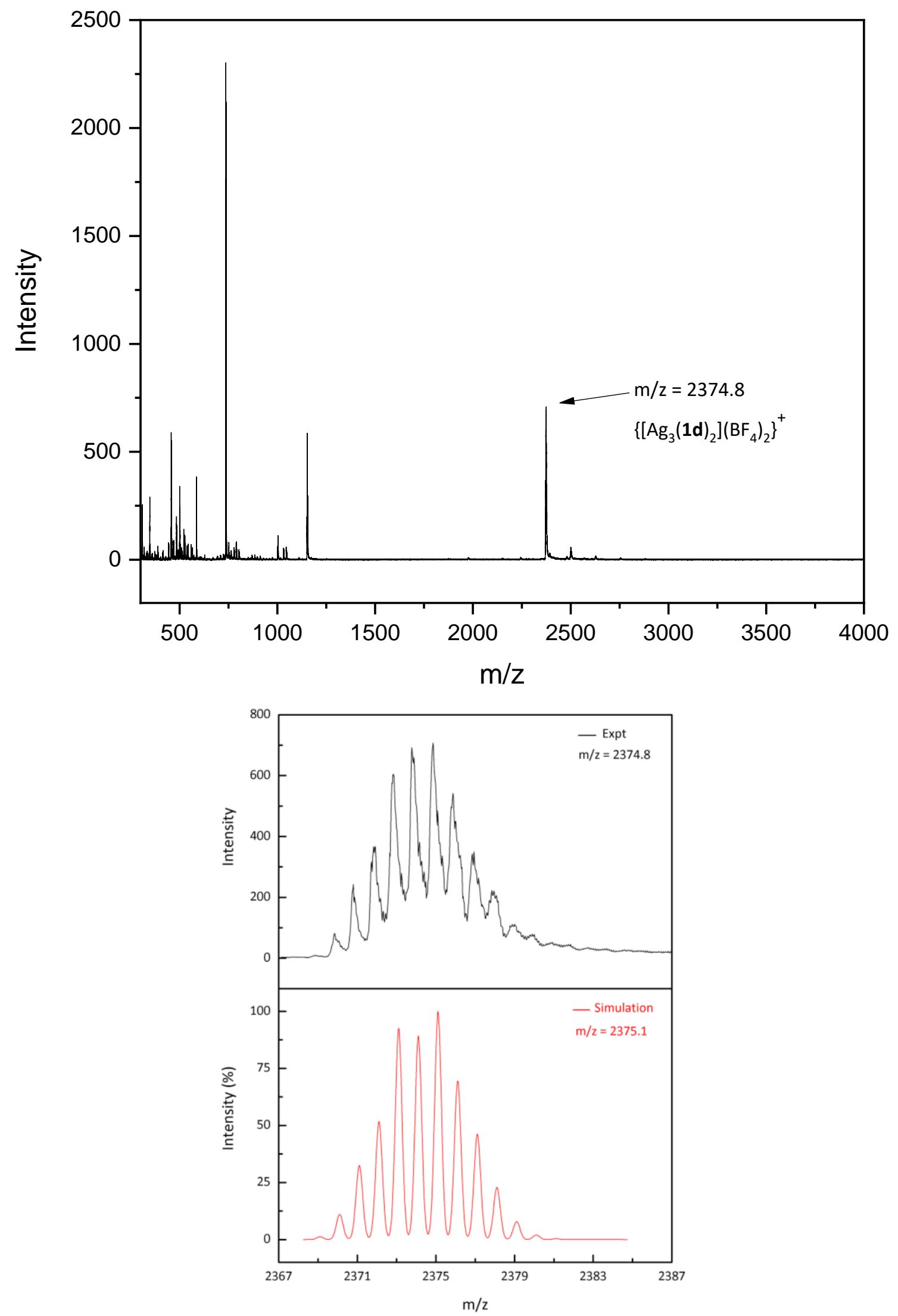

Figure S100. ESI mass spectrum of $\left[\mathrm{Ag}_{3}(\mathbf{1 d})_{2}\right]\left[\mathrm{BF}_{4}\right]_{3}$, recorded in $\mathrm{CH}_{3} \mathrm{CN}$ solution using positive ion mode, plus an expansion and isotope simulation of the parent ion. 

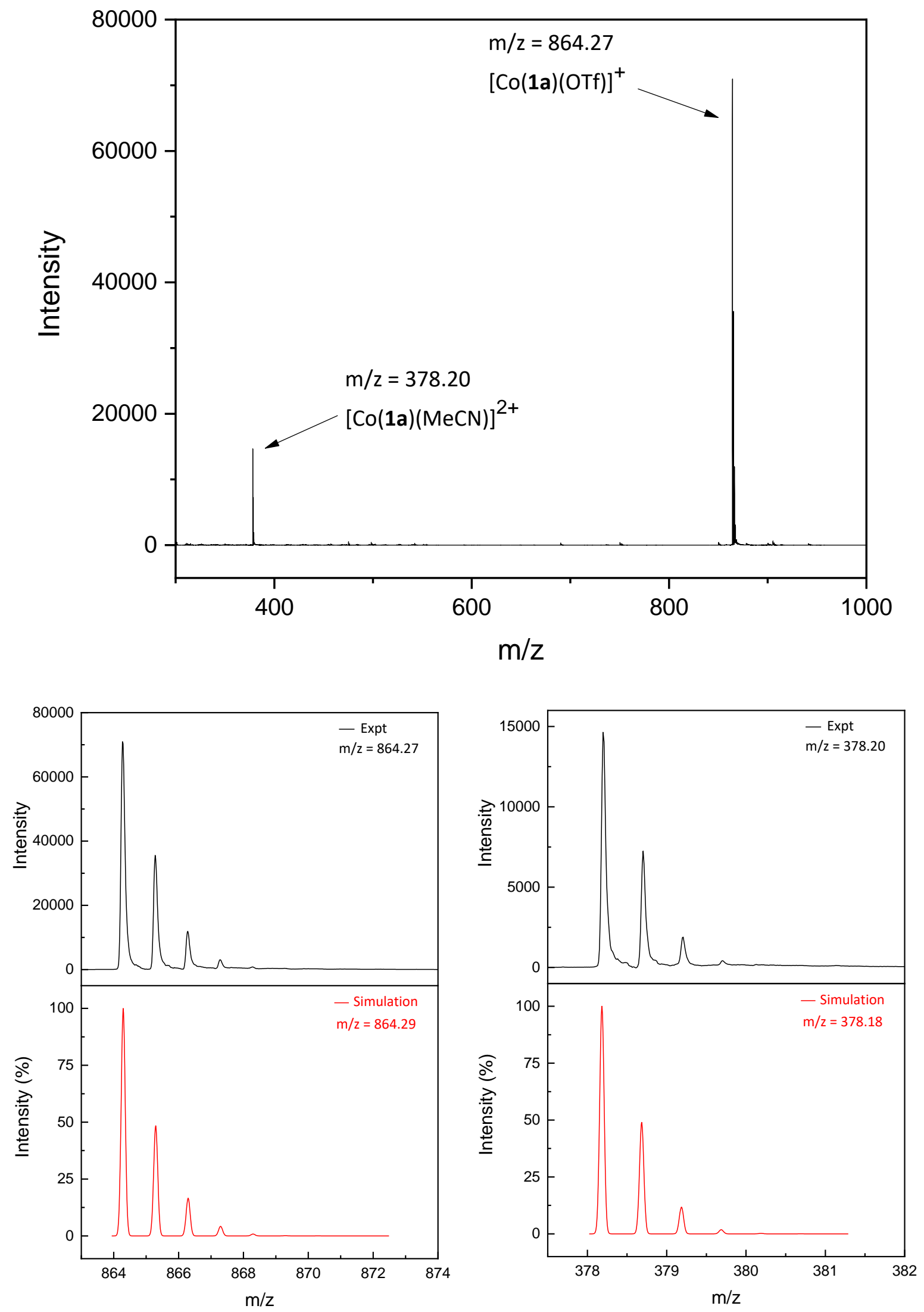

Figure S101. ESI mass spectrum of $[\mathrm{Co}(1 \mathrm{a})(\mathrm{MeCN})](\mathrm{OTf})_{2}$, recorded in $\mathrm{CH}_{3} \mathrm{CN}$ solution using positive ion mode, plus an expansion and isotope simulation of the parent ion. 

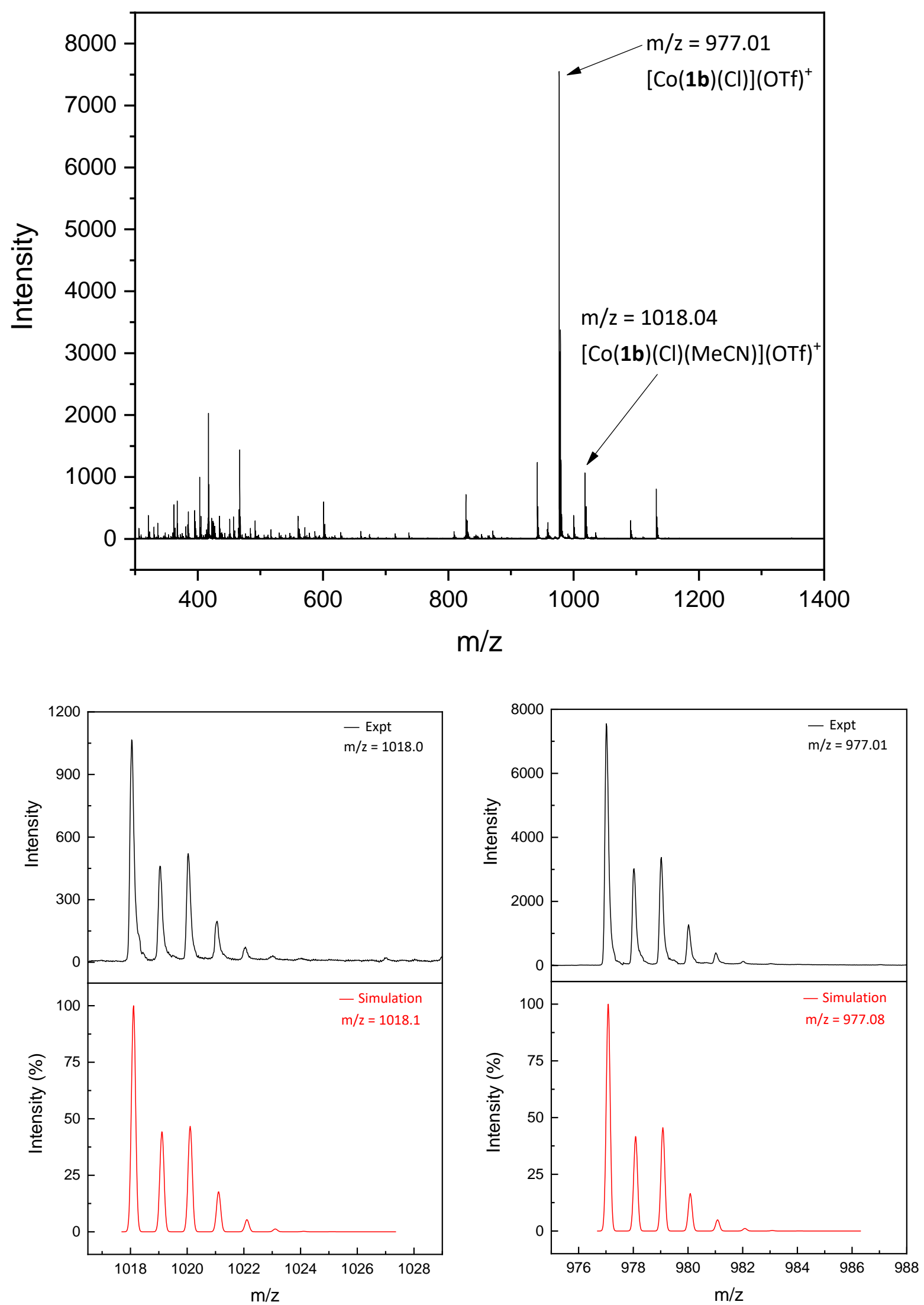

Figure S102. ESI mass spectrum of $[\mathrm{Co}(\mathbf{1 b})(\mathrm{Cl})(\mathrm{MeCN})](\mathrm{OTf})_{2}$, recorded in $\mathrm{CH}_{3} \mathrm{CN}$ solution using positive ion mode, plus an expansion and isotope simulation of the parent ion. 

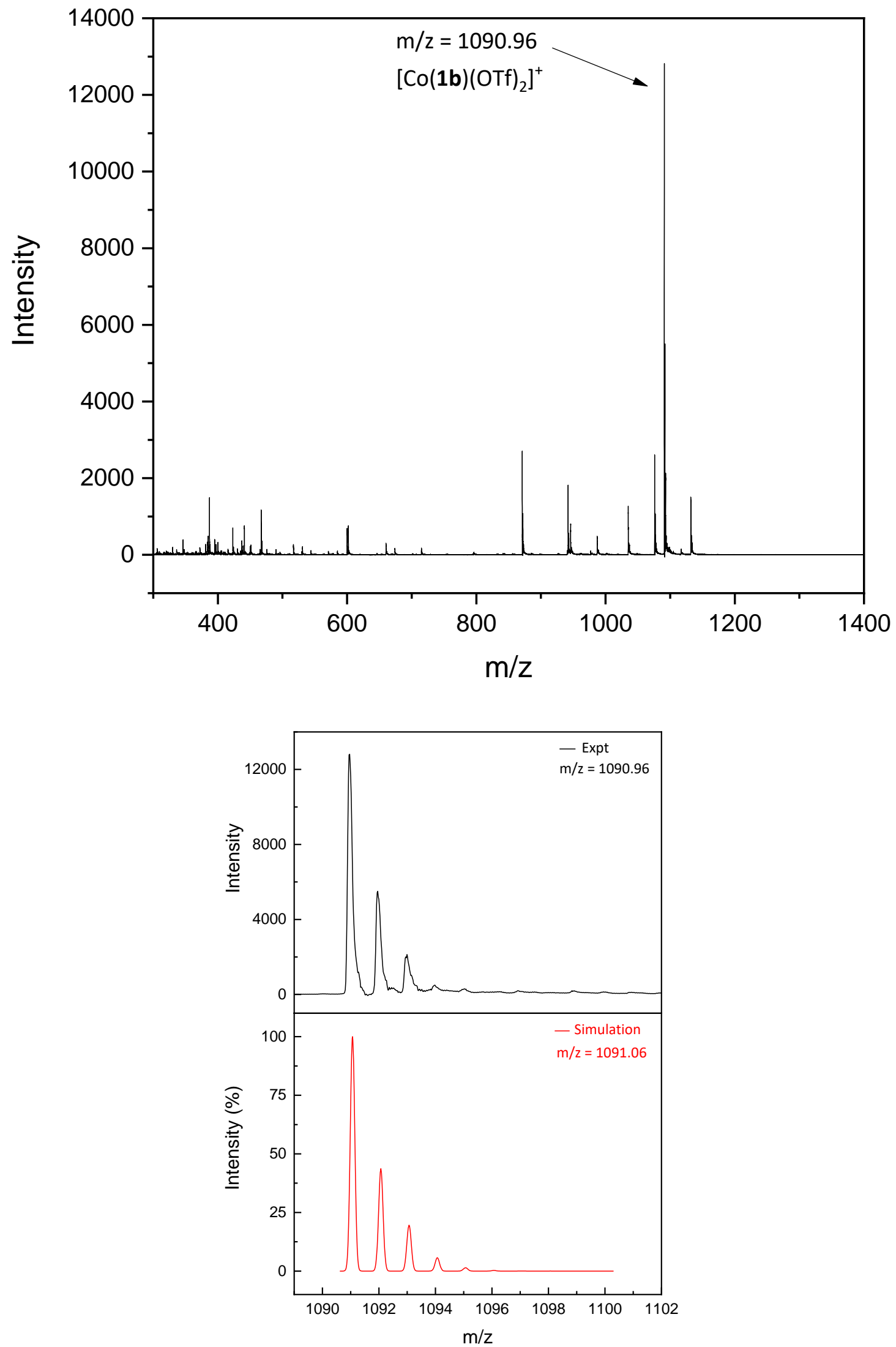

Figure S103. ESI mass spectrum of $\left[\mathrm{Co}(\mathbf{1 b})(\mathrm{MeCN})_{2}\right](\mathrm{OTf})_{3}$, recorded in $\mathrm{CH}_{3} \mathrm{CN}$ solution using positive ion mode, plus an expansion and isotope simulation of the parent ion. 


\section{$\underline{\text { X-ray Crystallography }}$}

Crystals of $[\mathbf{4 a}](\mathrm{OTf})_{3}, \quad[\mathbf{4 d}](\mathrm{OTf})_{3}, \quad\left[\mathrm{Ag}_{3}(\mathbf{1} \mathbf{a})_{2}\right](\mathrm{OTf})_{3}, \quad\left[\mathrm{Ag}_{3}(\mathbf{1} \mathbf{b})_{2}\right]\left(\mathrm{BF}_{4}\right)_{3}, \quad\left[\mathrm{Ag}_{3}(\mathbf{1 c})_{2}\right]\left(\mathrm{BF}_{4}\right)_{3}$, $\left[\mathrm{Ag}_{3}(\mathbf{1 d})_{2}\right](\mathrm{OTf})_{3},[\mathrm{Co}(\mathbf{1 a})(\mathrm{MeCN})](\mathrm{OTf})$, and $[\mathrm{Co}(\mathbf{1} \mathbf{b})(\mathrm{Cl})(\mathrm{MeCN})](\mathrm{OTf})_{2}$ were mounted on a Bruker X8 Quest CPAD area detector diffractometer and data was collected using an I $\mu \mathrm{S} 3.0$ Microfocus Mo-Ka radiation $(\lambda=0.71073 \AA$ A $)$. $\left[\mathrm{Co}(\mathbf{1 b})(\mathrm{MeCN})_{2}\right](\mathrm{OTf})_{3}$ was mounted on a Bruker Kappa Apex II area detector diffractometer and data was collected using an I $\mathrm{S}$ S 3.0 Microfocus Cu-K $\alpha$ radiation $(\lambda=1.54178 \AA$ ). Data reduction and absorption corrections were performed using the SAINT and SADABS software packages, respectively. ${ }^{[9]}$ All structures were solved by direct methods and refined via full-matrix least squares differences on $F^{2}$, using the SHELXT 2016 software package. ${ }^{[10,11]}$ All non-hydrogen atoms were refined anisotropically, after which hydrogen atoms were introduced at calculated positions and further refinement of data was performed. Renderings of the crystal structures were created using the ORTEP-3 software package. ${ }^{[12]}$ All crystallographic data associated with this manuscript have been deposited with, and can be obtained free of charge from, the Cambridge Crystallography Data Center (deposition numbers: CCDC 2013469 - 2013474, and 2058781- 2058783).

The following discussion is a continuation/elaboration of that provided in the main manuscript. $\mathrm{Ag}-\mathrm{Ag}$ bonding in $\left[\mathrm{Ag}_{3}(\mathbf{1 b})\right]^{3+}$ causes a perturbation of its structural parameters from those of the other silver complexes, which are very similar to one another. As might be expected, the average $\mathrm{Ag}-\mathrm{C}$ bond length in $\left[\mathrm{Ag}_{3}(\mathbf{1} \mathbf{b})\right]^{3+}$ is slightly longer $(2.113 \AA)$ than the corresponding, essentially identical, values observed for the complexes supported by $\mathbf{1 a}, \mathbf{1} \mathbf{c}$, and $1 \mathbf{d}(2.083,2.075$ and $2.079 \AA$, respectively). Similarly, the average $\mathrm{C}-\mathrm{Ag}-\mathrm{C}$ bond angles in all four silver complexes are close to $180^{\circ}$, with the largest deviation from linearity being seen for ligand $\mathbf{1 b}$, for which the average value is $164.4^{\circ}$. Those of the other complexes ligated by 1a, 1 c, and $1 \mathbf{d}$ are $176.0,177.7$, and $175.9^{\circ}$, respectively.

In the complexes $\left[\mathrm{Ag}_{3}(\mathbf{1 a})\right]^{3+},\left[\mathrm{Ag}_{3}(\mathbf{1} \mathbf{c})\right]^{3+}$, and $\left[\mathrm{Ag}_{3}(\mathbf{1 d})\right]^{3+}$, the average separation of the carbenic donor atoms (C...C), within each ligand (5.966, 5.92 and $5.980 \AA$, respectively), are comparable to their respective $\mathrm{Ag} \cdot \cdots \mathrm{Ag}$ distances. In contrast, the corresponding average $\mathrm{C} \cdot \mathrm{C} \mathrm{C}$ distance in $\left[\mathrm{Ag}_{3}(\mathbf{1 b})\right]^{3+}$, of $4.51 \AA$, is significantly longer than the average $\mathrm{Ag}-\mathrm{Ag}$ bond length. To accommodate this difference, the $\mathrm{C}$ donors twist away from the $\mathrm{Ag}$ ions. This is best viewed by looking down the near linear axis comprised of the centroid of the three $\mathrm{Ag}$ atoms $\left(\mathrm{Ag}_{\text {cent }}\right)$ 
and the centroids of the three donor $\mathrm{C}$-atoms $\left(\mathrm{C}_{\text {cent }}\right)$ of each ligand $\left(\mathrm{C}_{\text {cent }} \cdots A \mathrm{Ag}_{\text {cent }} \cdots \mathrm{C}_{\text {cent }}\right.$ angles vary between 170.68 and $178.89^{\circ}$ ). When the complexes of $\mathbf{1 a}, \mathbf{1 c}$ and $\mathbf{1 d}$ are viewed from this perspective, the $\mathrm{Ag}$ ions and $\mathrm{C}$ atoms directly coordinated to them are nearly eclipsed with respect to one another (Figures S93, S95 and S96). However, they are clearly staggered with respect to one another in $\left[\mathrm{Ag}_{3}(\mathbf{1 b})\right]^{3+}$ (Figure S94). This can be quantified using the $\mathrm{C} \cdots \mathrm{C}_{\text {cent }} \cdots \mathrm{Ag}_{\text {cent }} \cdots \mathrm{Ag}$ torsion angles of the individual $\mathrm{Ag}-\mathrm{C}$ bonds, which have average values of near zero in $\left[\mathrm{Ag}_{3}(\mathbf{1 a})\right]^{3+},\left[\mathrm{Ag}_{3}(\mathbf{1 c})\right]^{3+}$, and $\left[\mathrm{Ag}_{3}(\mathbf{1 d})\right]^{3+}\left(1.05,4.24\right.$ and $4.88^{\circ}$, respectively) and $36.93^{\circ}$ in $\left[\mathrm{Ag}_{3}(\mathbf{1 b})\right]^{3+}$. As a consequence, $\left[\mathrm{Ag}_{3}(\mathbf{1 b})\right]^{3+}$ has a more compact structure, with an average $A g_{\text {cent }} \cdots C_{\text {cent }}$ separation of $1.386 \AA$, whereas those of the other complexes are between 2.057 and $2.218 \AA$.

In all four complexes, the arms of the two tridentate ligands form propellers of opposing orientation. As a result, the Trz donors of the two ligands are staggered with respect to one another. This is evident from the angles between the mean planes of the two Trz rings coordinated to each $\mathrm{Ag}^{\prime}$ ion; the complexes supported by $\mathbf{1 a}, \mathbf{1 b}, \mathbf{1} \mathbf{c}$ and $\mathbf{1 d}$ have average values of $38.07,75.80,26.32$, and $28.91^{\circ}$, respectively. The larger value for $\left[\operatorname{Ag}_{3}(\mathbf{1} \mathbf{b})\right]^{3+}$ is a direct result of its more compact structure, which also impacts the orientation of the electron lone pair on the tertiary amine centres of the ligands. $\ln \left[\mathrm{Ag}_{3}(\mathbf{1} \mathbf{b})\right]^{3+}$ they point towards the centre of the sandwich complex, whereas they point in the opposite direction in the others. 


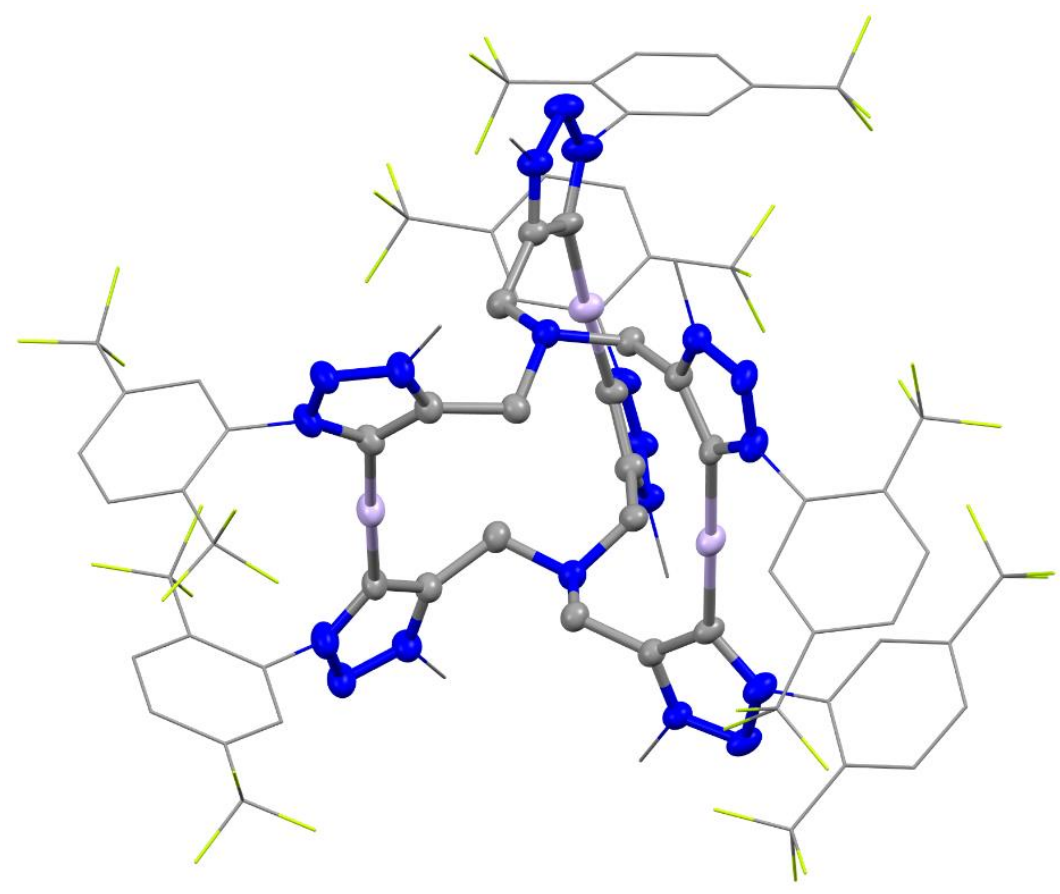

Figure S104. X-ray crystal structure of $\left[\mathrm{Ag}_{3}\left(\mathbf{1 C}_{2}\right]\left(\mathrm{BF}_{4}\right)_{3}\right.$. Whereas the methyl and aryl substituents are rendered as sticks, the remainder is depicted using $20 \%$ probability thermal ellipsoids. For clarity, hydrogen atoms, counterions and solvent molecules are omitted. Atom colour scheme: Ag, light purple-pink; C, grey; F, yellow-green; N, blue. 


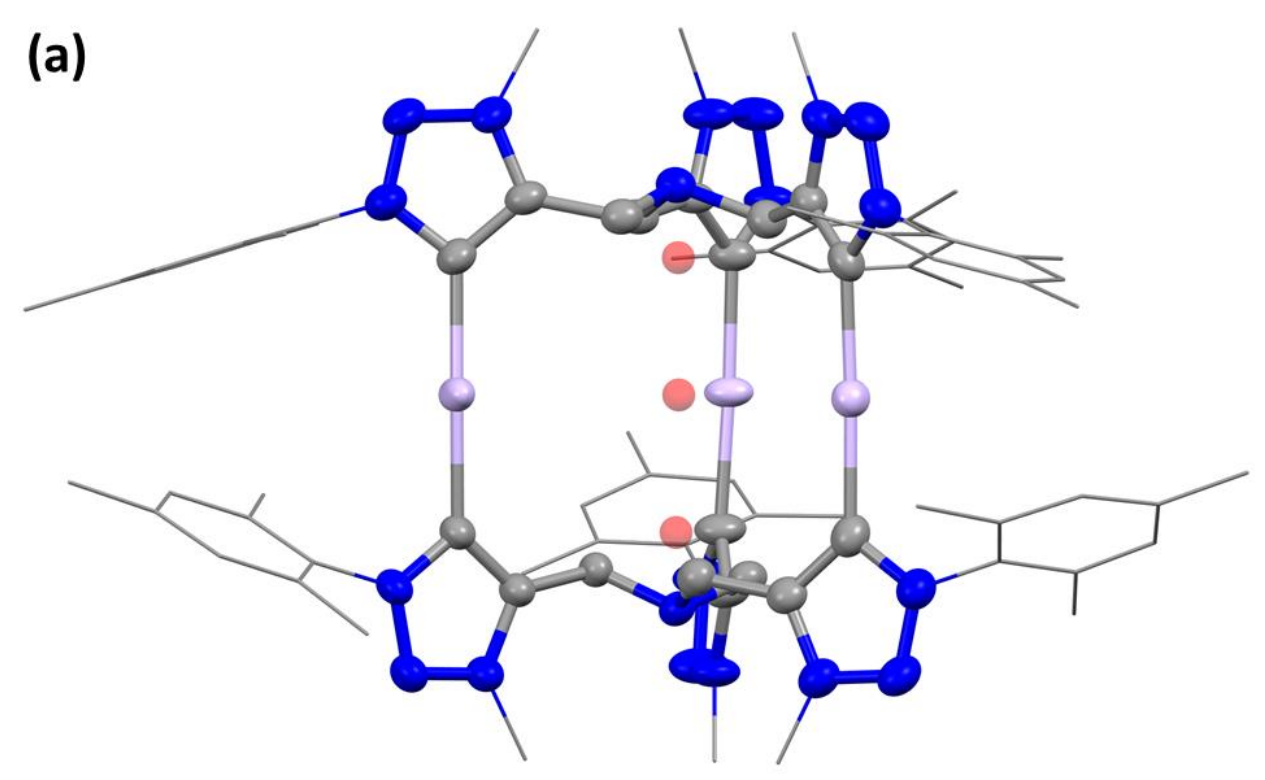

(b)

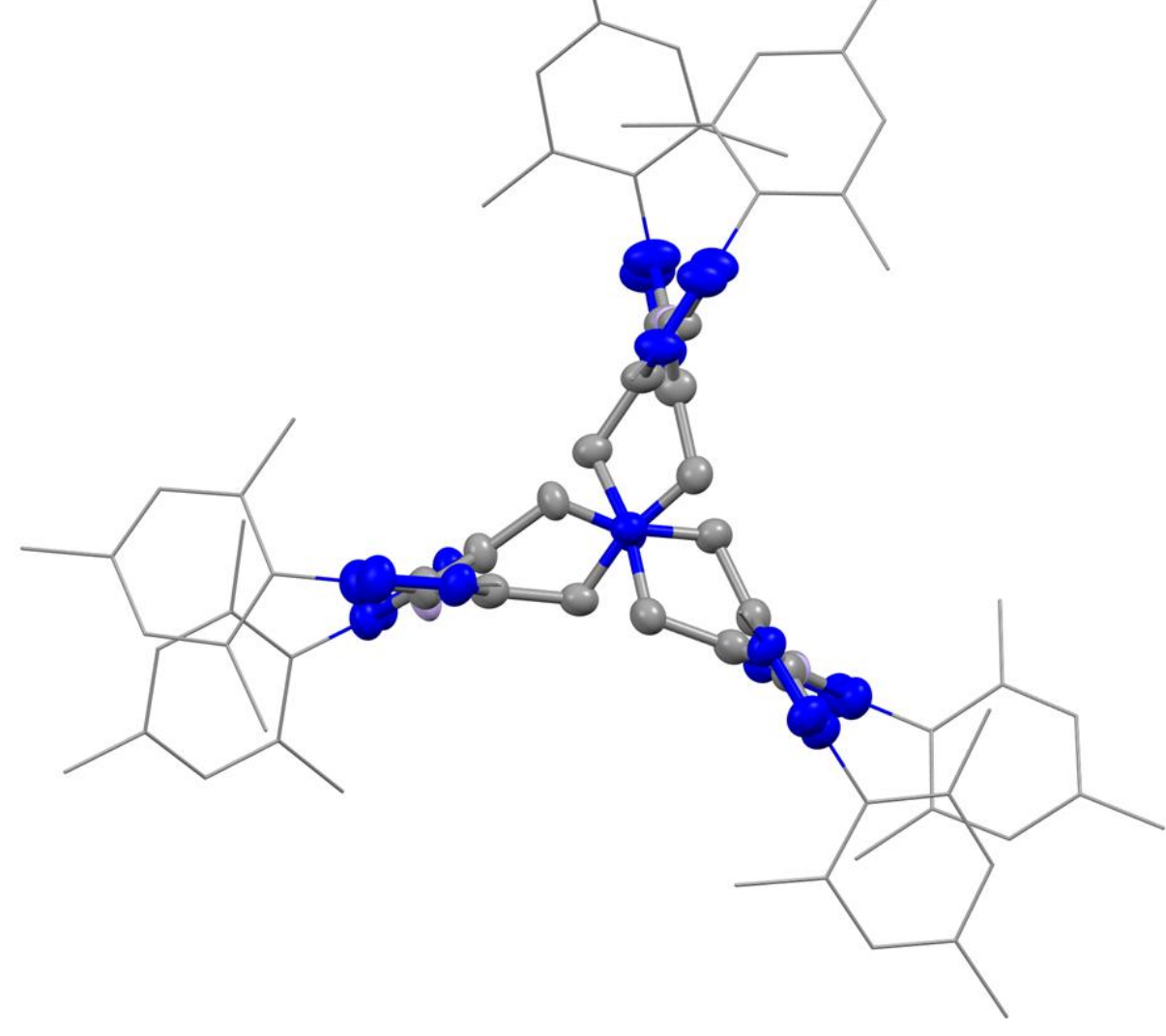

Figure S105. X-ray crystal structure of $\left[\mathrm{Ag}_{3}(1 \mathrm{a})_{2}\right](\mathrm{OTf})_{3}$, showing the $\mathrm{C}_{\text {cent }}, \mathrm{Ag}_{\text {cent }}$ and $\mathrm{C}_{\text {cent }}$ centroids (red spheres), viewed from the (a) side and (b) above. Whereas the methyl and aryl substituents are rendered as sticks, the remainder is depicted using $50 \%$ probability thermal ellipsoids. For clarity, hydrogen atoms, counterions and solvent molecules are omitted. Atom colour scheme: Ag, light purple-pink; C, grey; N, blue. 

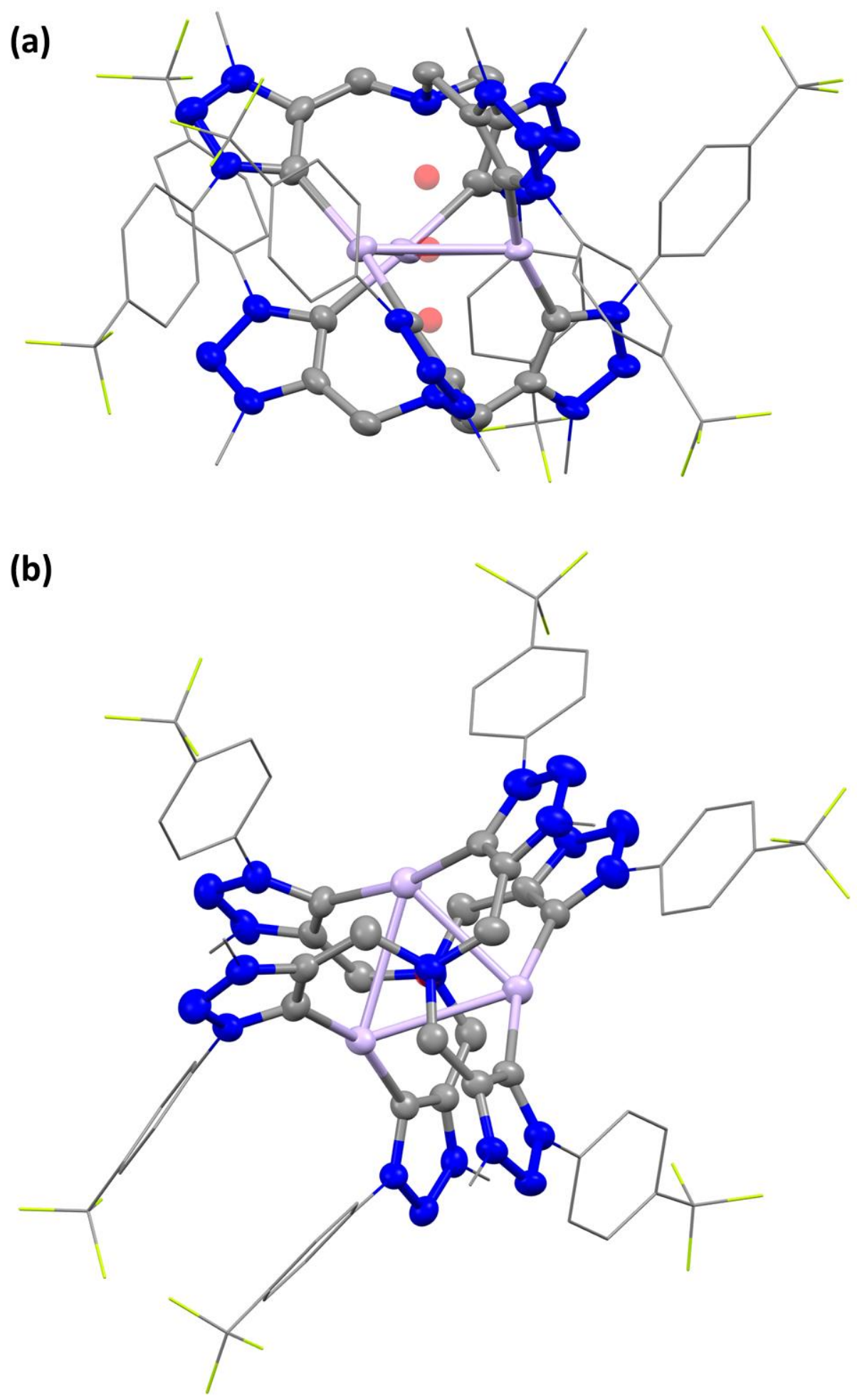

Figure S106. X-ray crystal structure of $\left[\mathrm{Ag}_{3}(\mathbf{1 b})_{2}\right]\left(\mathrm{BF}_{4}\right)_{3}$, showing the $\mathrm{C}_{\text {cent, }}, \mathrm{Ag}_{\text {cent }}$ and $\mathrm{C}_{\text {cent }}$ centroids (red spheres), viewed from the (a) side and (b) above. Whereas the methyl and aryl substituents are rendered as sticks, the remainder is depicted using $50 \%$ probability thermal ellipsoids. For clarity, hydrogen atoms, counterions and solvent molecules are omitted. Atom colour scheme: Ag, light purple-pink; C, grey; F, yellow-green; N, blue. 


\section{(a)}
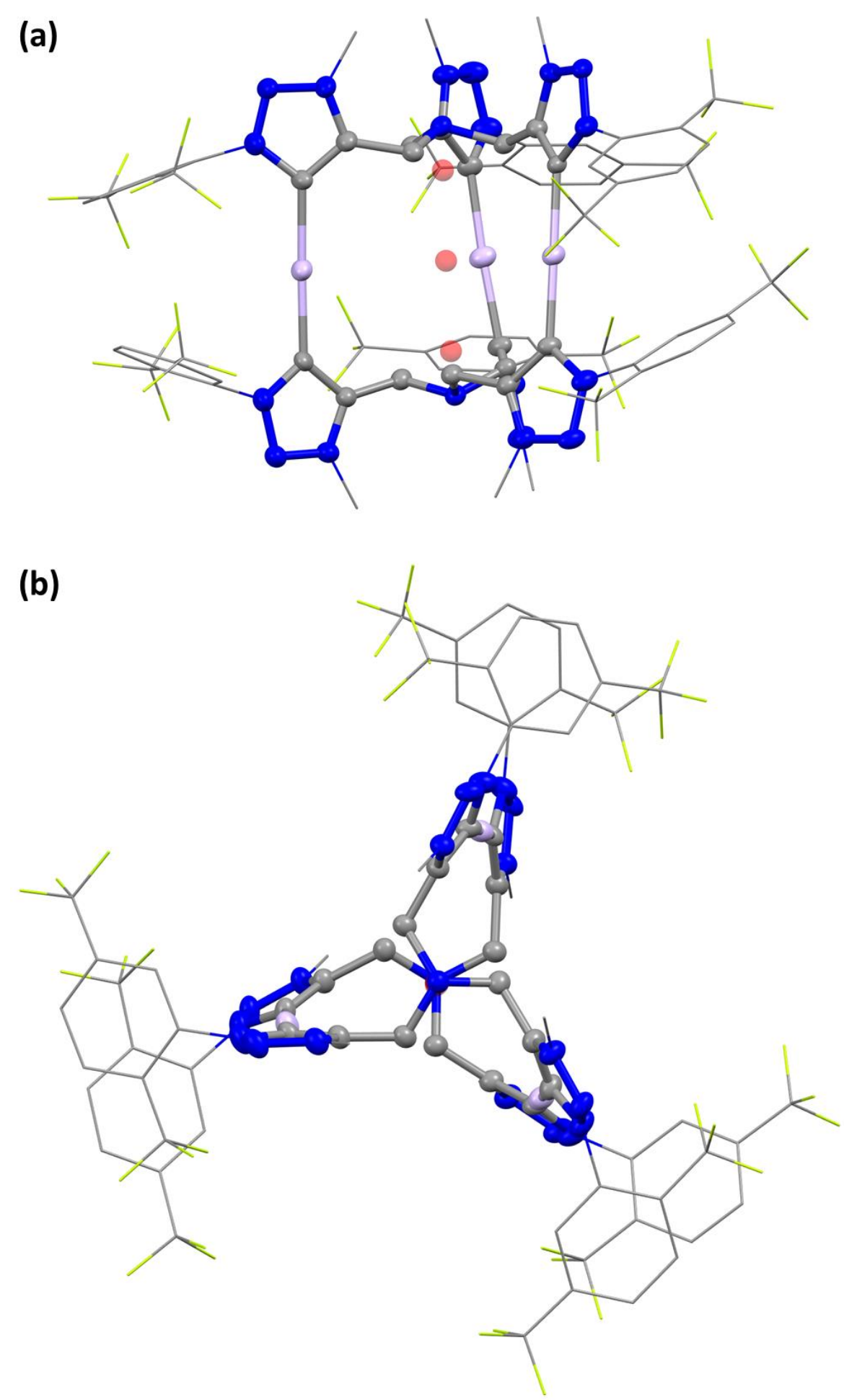

Figure S107. X-ray crystal structure of $\left[\mathrm{Ag}_{3}(\mathbf{1 c})_{2}\right]\left(\mathrm{BF}_{4}\right)_{3}$, showing the $\mathrm{C}_{\text {cent }}, \mathrm{Ag}_{\text {cent }}$ and $\mathrm{C}_{\text {cent }}$ centroids (red spheres), viewed from the (a) side and (b) above. Whereas the methyl and aryl substituents are rendered as sticks, the remainder is depicted using $20 \%$ probability thermal ellipsoids. For clarity, hydrogen atoms, counterions and solvent molecules are omitted. Atom colour scheme: Ag, light purple-pink; C, grey; F, yellow-green; N, blue. 


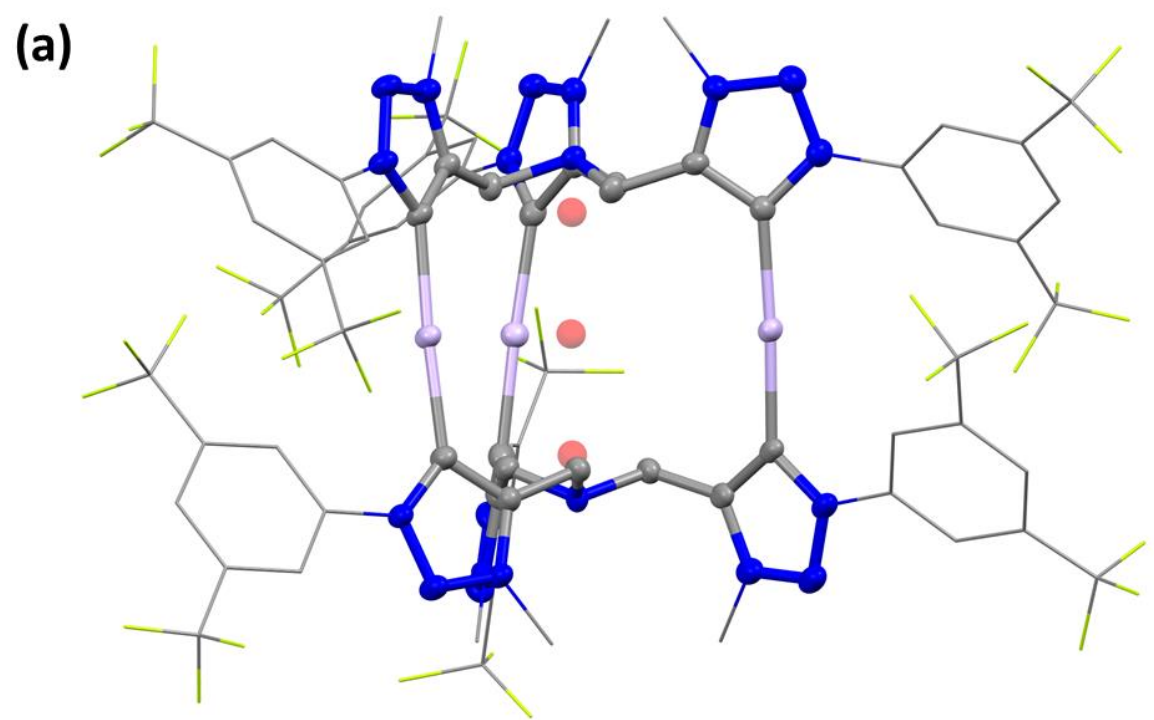

(b)

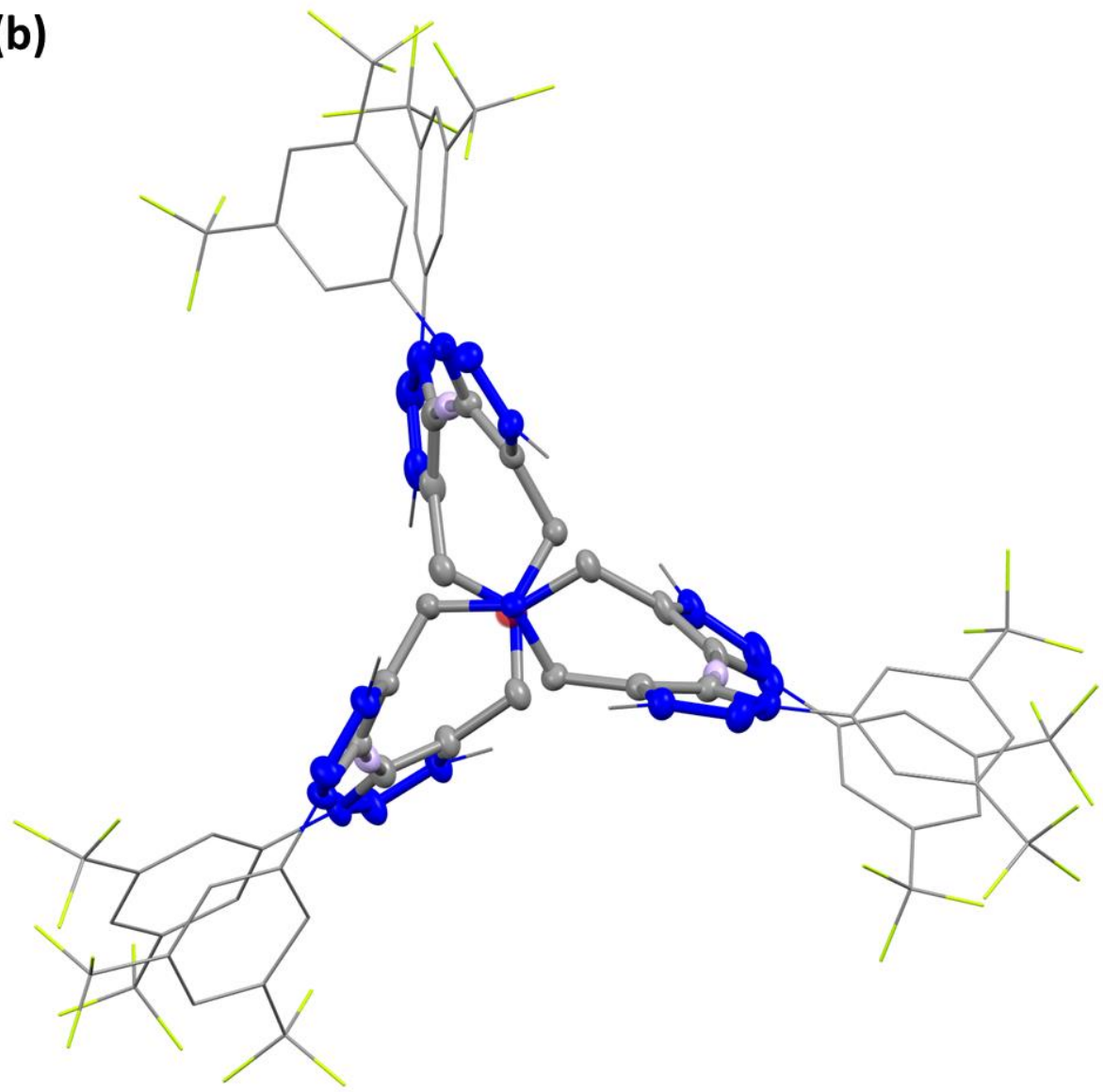

Figure S108. X-ray crystal structure of $\left[\mathrm{Ag}_{3}(1 \mathrm{~d})_{2}\right](\mathrm{OTf})_{3}$, showing the $\mathrm{C}_{\text {cent }}, \mathrm{Ag}_{\text {cent }}$ and $\mathrm{C}_{\text {cent }}$ centroids (red spheres), viewed from the (a) side and (b) above. Whereas the methyl and aryl substituents are rendered as sticks, the remainder is depicted using $50 \%$ probability thermal ellipsoids. For clarity, hydrogen atoms, counterions and solvent molecules are omitted. Atom colour scheme: Ag, light purple-pink; C, grey; F, yellow-green; N, blue. 
(a)

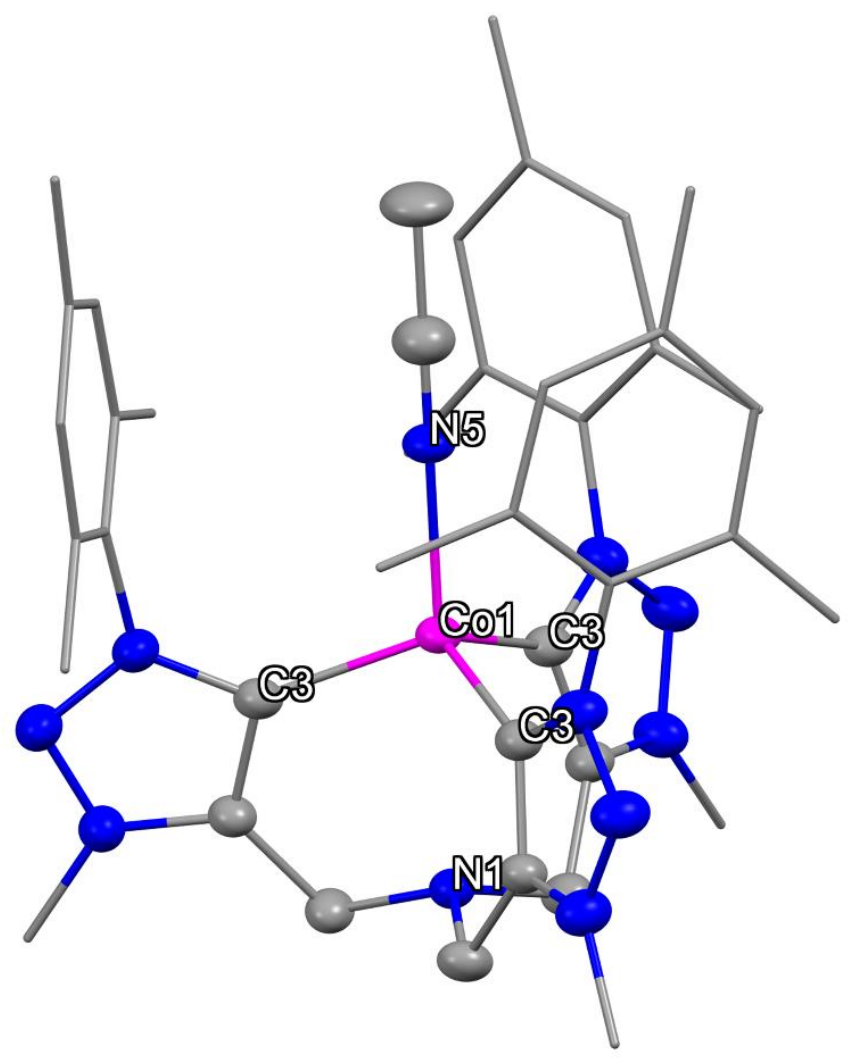

(b)

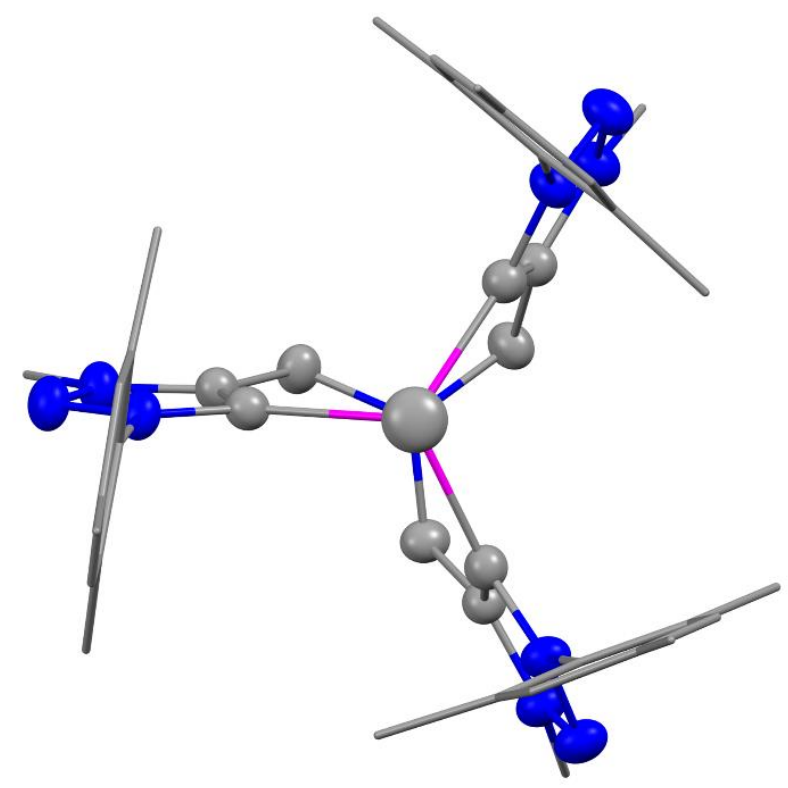

Figure S109. X-ray crystal structure of $[\mathrm{Co}(1 \mathrm{a})(\mathrm{NCMe})](\mathrm{OTf})_{2}$, (a) with atom labelling and (b) viewed from above. There are three distinct molecules per unit cell, but only one of them is included here. Whereas the methyl and aryl substituents are rendered as sticks, the remainder is depicted using $50 \%$ probability thermal ellipsoids. For clarity, hydrogen atoms, counterions and solvent molecules are omitted. Atom colour scheme: $\mathrm{C}$, grey; $\mathrm{Co}$, magenta; $\mathrm{N}$, blue. 

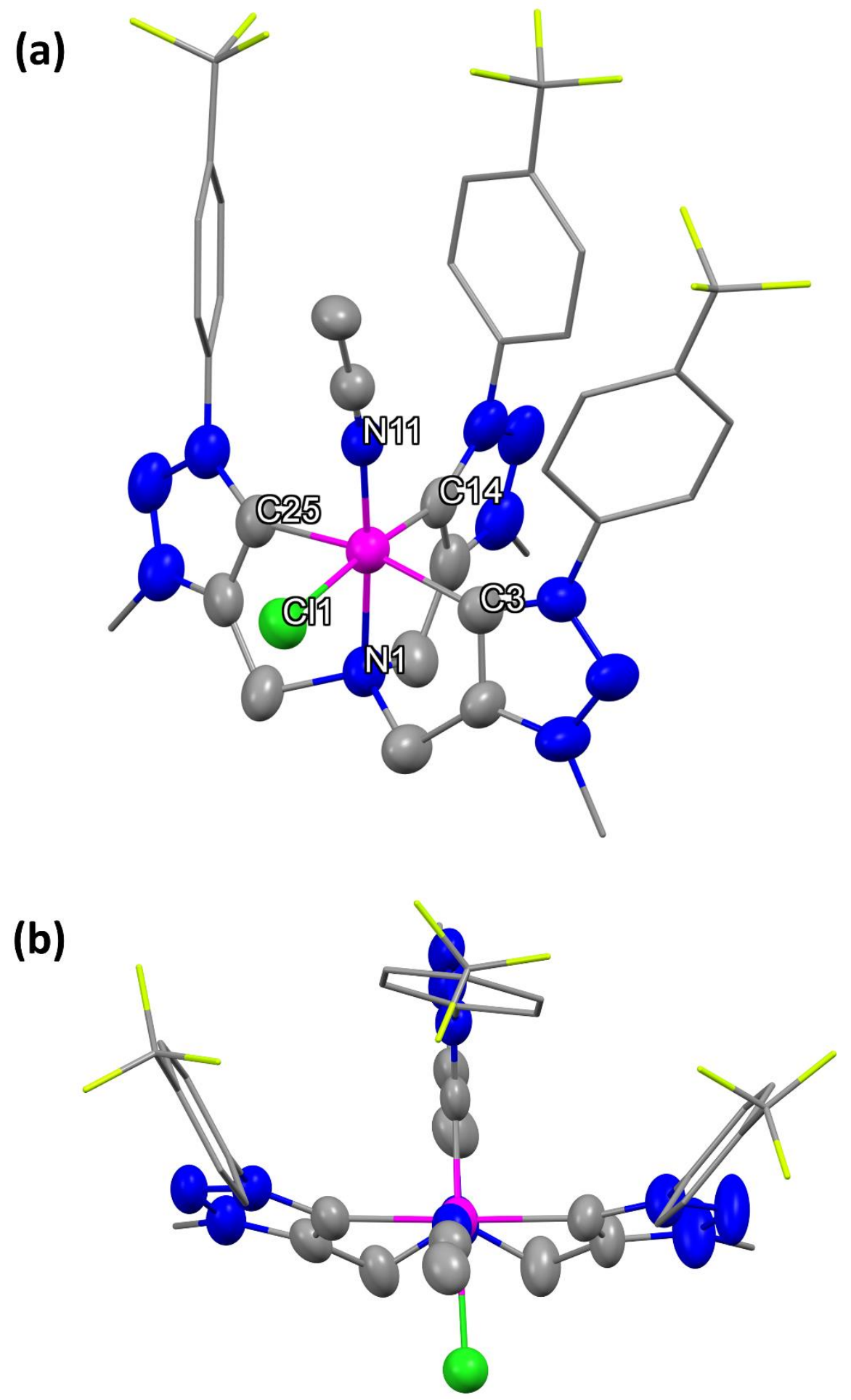

Figure S110. X-ray crystal structure of $[\mathrm{Co}(\mathbf{1 b})(\mathrm{Cl})(\mathrm{NCMe})](\mathrm{OTf})_{2}$, (a) with atom labelling and (b) viewed from above. Whereas the methyl and aryl substituents are rendered as sticks, the remainder is depicted using $50 \%$ probability thermal ellipsoids. For clarity, hydrogen atoms, counterions and solvent molecules are omitted. Atom colour scheme: $\mathrm{C}$, grey; $\mathrm{Cl}$, green; $\mathrm{Co}$, magenta; $\mathrm{F}$, yellow-green; $\mathrm{N}$, blue. 
(a)

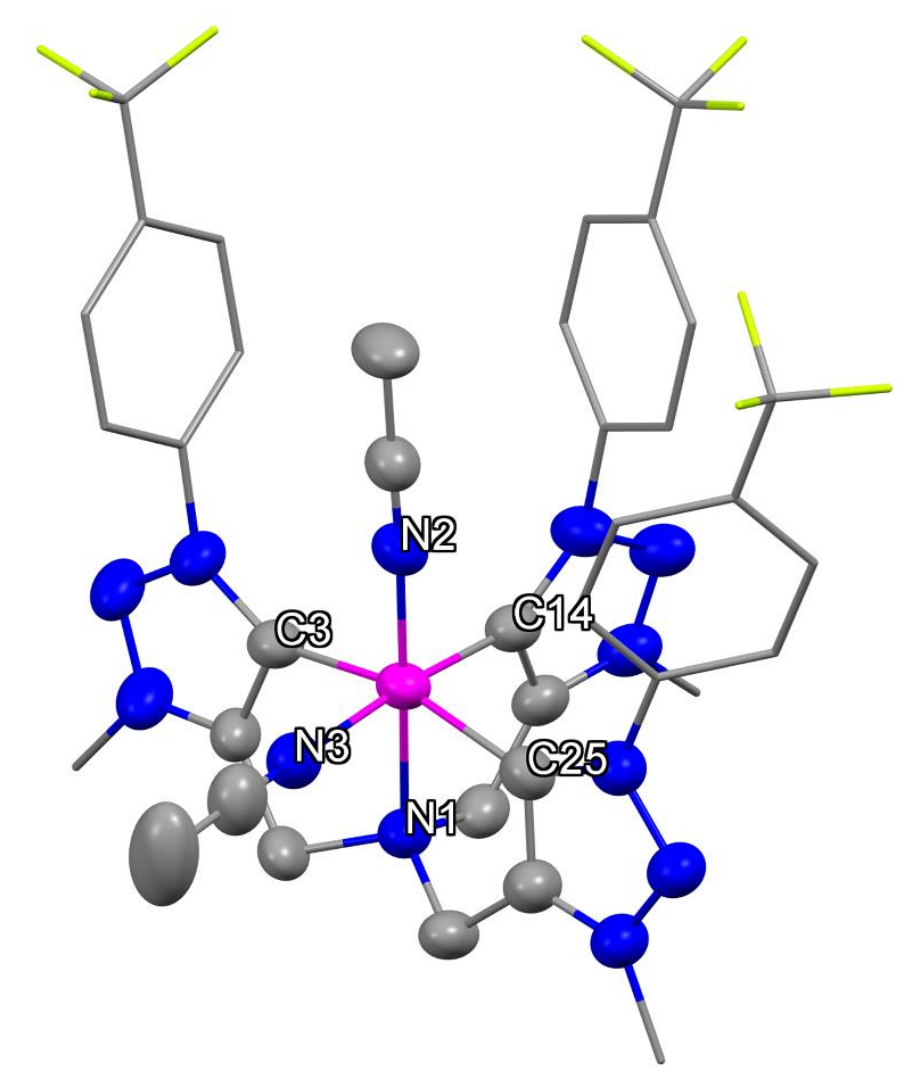

(b)

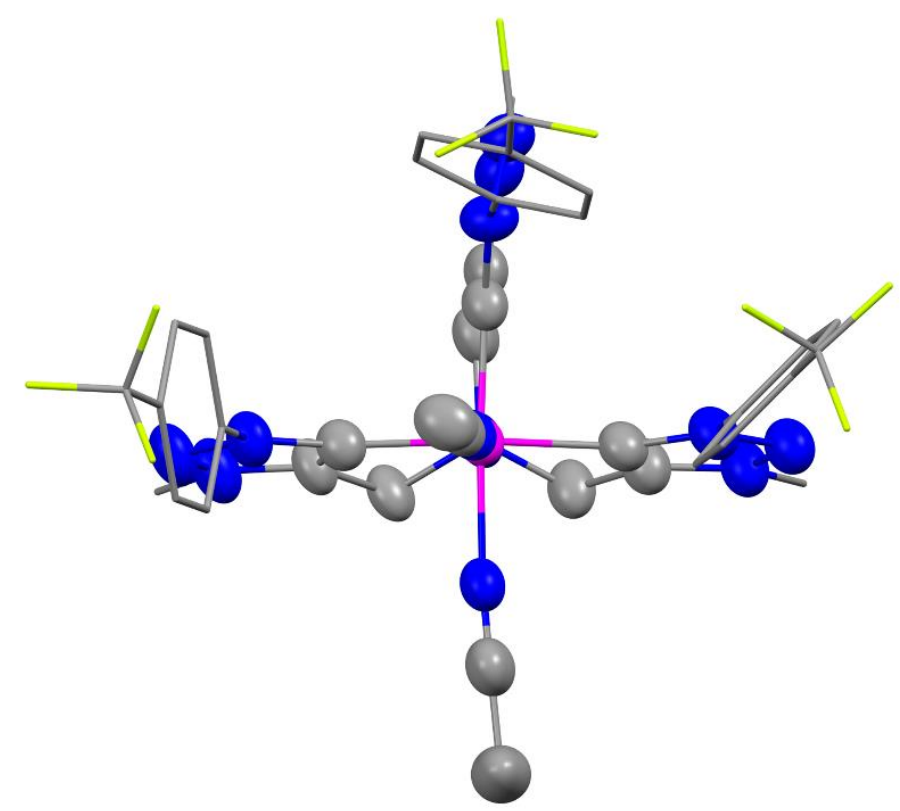

Figure S111. X-ray crystal structure of $\left[\mathrm{Co}(\mathbf{1 b})\left(\mathrm{NCMe}_{2}\right](\mathrm{OTf})_{3},(a)\right.$ with atom labelling and (b) viewed from above. Whereas the methyl and aryl substituents are rendered as sticks, the remainder is depicted using $50 \%$ probability thermal ellipsoids. For clarity, hydrogen atoms, counterions and solvent molecules are omitted. Atom colour scheme: $\mathrm{C}$, grey; $\mathrm{Co}$, magenta; F, yellow-green; $\mathrm{N}$, blue. 
Table S1. Crystallographic and structure refinement data for [4a](OTf $)_{3}$ and [4d](OTf $)_{3}$.

\begin{tabular}{|c|c|c|}
\hline Compounds & {$[4 a](O T f)_{3}$} & {$[4 d](O T f)_{3}$} \\
\hline Appearance & colorless block & colorless plate \\
\hline Formula & $\mathrm{C}_{46} \mathrm{H}_{59} \mathrm{~F}_{9} \mathrm{~N}_{11} \mathrm{O}_{9.50} \mathrm{~S}_{3}$ & $\mathrm{C}_{43} \mathrm{H}_{37} \mathrm{~F}_{27} \mathrm{~N}_{10} \mathrm{O}_{10} \mathrm{~S}_{3}$ \\
\hline$F W, \mathrm{~g} \mathrm{~mol}^{-1}$ & 1185.22 & $1463.00 \mathrm{~g} / \mathrm{mol}$ \\
\hline Cryst. Syst. & triclinic & triclinic \\
\hline Space group & $P-1$ & $P-1$ \\
\hline Size, $\mathrm{mm}^{3}$ & $0.160 \times 0.180 \times 0.220$ & $0.080 \times 0.240 \times 0.320$ \\
\hline$T, \mathrm{~K}$ & $100(2)$ & $100(2)$ \\
\hline Wavelength, Å & 0.71073 & 0.71073 \\
\hline$a, \AA$ & $12.9008(3)$ & $8.6811(5)$ \\
\hline$b, \AA$ & $15.7537(3)$ & $14.9167(10)$ \\
\hline$c, \AA$ & $15.9230(4)$ & $22.1204(16)$ \\
\hline$\alpha$, deg & $114.4602(7)$ & $90.603(2)$ \\
\hline$\beta, \operatorname{deg}$ & $96.5585(9)$ & $98.746(2)$ \\
\hline$\gamma$, deg & $104.0742(8)$ & $93.010(2)$ \\
\hline$V, \AA^{3}$ & $2771.32(11)$ & $2826.7(3)$ \\
\hline$Z$ & 2 & 2 \\
\hline$d_{\text {calc., }} \mathrm{g} \mathrm{cm}^{-3}$ & 1.420 & 1.719 \\
\hline$\mu, \mathrm{mm}^{-1}$ & 0.227 & 0.285 \\
\hline Refl. collected & 88630 & 44452 \\
\hline GOF & 1.083 & 1.024 \\
\hline$\Delta / \sigma_{\max }$ & 0.004 & 0.001 \\
\hline$R ; I>2 \sigma(I)$ & 0.0621 & 0.0889 \\
\hline $\begin{array}{l}\text { Largest diff. peak and } \\
\text { hole, } e \AA^{-3}\end{array}$ & $1.633 /-0.854$ & $1.324 /-0.878$ \\
\hline $\begin{array}{l}\text { R.M.S. deviation from } \\
\text { mean. } \AA^{-3}\end{array}$ & 0.066 & 0.123 \\
\hline
\end{tabular}


Table S2. Crystallographic and structure refinement data for $\left.\left[\mathrm{Ag}_{3}(\mathbf{1 a})_{2}\right](\mathrm{OTf})_{3},\left[\mathrm{Ag}_{3}(\mathbf{1} \mathbf{b})_{2}\right]\left(\mathrm{BF}_{4}\right)_{3},\left[\mathrm{Ag}_{3}(\mathbf{1 c})_{2}\right]_{(\mathrm{BF}}\right)_{3}$, and $\left[\mathrm{Ag}_{3}(\mathbf{1} \mathbf{d})_{2}\right](\mathrm{OTf})_{3}$.

\begin{tabular}{|c|c|c|c|c|}
\hline Compounds & {$\left[\mathrm{Ag}_{3}(1 \mathrm{a})_{2}\right](\mathrm{OTf})_{3}$} & {$\left[\mathrm{Ag}_{3}(\mathbf{1 b})_{2}\right]\left(\mathrm{BF}_{4}\right)_{3}$} & {$\left[\mathrm{Ag}_{3}(1 \mathrm{C})_{2}\right](\mathrm{BF} 4)_{3}$} & {$\left[\mathrm{Ag}_{3}(1 \mathrm{dd})_{2}\right](\mathrm{OTf})_{3}$} \\
\hline Appearance & colorless block & colorless plate & colorless block & colorless plate \\
\hline Formula & $\mathrm{C}_{85} \mathrm{H}_{102} \mathrm{Ag}_{3} \mathrm{~F}_{9} \mathrm{~N}_{22} \mathrm{O}_{9} \mathrm{~S}_{3}$ & $\mathrm{C}_{77.25} \mathrm{H}_{80} \mathrm{Ag}_{3} \mathrm{~B}_{3} \mathrm{~F}_{30} \mathrm{~N}_{23.50} \mathrm{O}_{3.75}$ & $\mathrm{C}_{72} \mathrm{H}_{48} \mathrm{Ag}_{3} \mathrm{~B}_{3} \mathrm{~F}_{48} \mathrm{~N}_{20}$ & $\mathrm{C}_{79} \mathrm{H}_{54} \mathrm{Ag}_{3} \mathrm{~F}_{45} \mathrm{~N}_{22} \mathrm{O}_{9} \mathrm{~S}_{3}$ \\
\hline$F W, \mathrm{~g} \mathrm{~mol}^{-1}$ & 2166.67 & 2323.68 & 2461.34 & 2730.23 \\
\hline Cryst. Syst. & monoclinic & tetragonal & monoclinic & triclinic \\
\hline Space group & P $12 / n 1$ & $\mathrm{P} 4 / \mathrm{n}$ & C $12 / c 1$ & $P-1$ \\
\hline Size $\left(\mathrm{mm}^{3}\right)$ & $0.220 \times 0.240 \times 0.280$ & $0.020 \times 0.120 \times 0.240$ & $0.240 \times 0.300 \times 0.420$ & $0.060 \times 0.120 \times 0.140$ \\
\hline$T, \mathrm{~K}$ & $100(2)$ & $100(2)$ & $100(2)$ & $100(2)$ \\
\hline Wavelength, Å & 0.71073 & 0.71073 & 0.71073 & 0.71073 \\
\hline$a, \AA$ & $18.9089(7)$ & $40.583(3)$ & $19.7822(13)$ & $13.8010(4)$ \\
\hline$b, \AA$ & $23.2641(6)$ & $40.583(3)$ & $34.184(2)$ & 19.8401(6) \\
\hline$c, \AA$ & 25.7911(9) & $11.8012(8)$ & $25.6517(16)$ & $21.4321(7)$ \\
\hline$\alpha, \operatorname{deg}$ & 90 & 90 & 90 & $82.2560(10)$ \\
\hline$\beta, \operatorname{deg}$ & $98.0210(11)$ & 90 & $112.610(2)$ & $79.1830(10)$ \\
\hline$\gamma, \operatorname{deg}$ & 90 & 90 & 90 & $70.2680(10)$ \\
\hline$V, \AA^{3}$ & $11234.5(6)$ & 19436.(3) & $16013.4(18)$ & $5409.5(3)$ \\
\hline
\end{tabular}


1.281

1.588

calc., $\mathrm{g} \mathrm{cm}^{-3}$

0.645

0.715

1.021

1.676

Refl. collected

101012

77402

0.450

0.734

GOF

1.022

1.034

110128

109624

$\Delta / \sigma_{\max }$

0.001

0.002

1.047

1.027

$R ; \mid>2 \sigma(I)$

0.0671

0.0828

0.004

0.002

Largest diff. peak and 2.930/-1.489

hole, $\mathrm{e} \AA^{-3}$

R.M.S. deviation from 0.109

$1.058 /-0.911$

0.1108

0.0688

mean, $\mathrm{e}^{-3}$

$1.132 /-0.880$

0.112

0.144

0.138 
Table S3. Crystallographic and structure refinement data of cobalt complexes.

\begin{tabular}{|c|c|c|c|}
\hline Compounds & {$[\mathrm{Co}(\mathrm{la})(\mathrm{MeCN})](\mathrm{OTf})_{2}$} & {$[\mathrm{Co}(1 \mathrm{~b})(\mathrm{Cl})(\mathrm{MeCN})](\mathrm{OTf})_{2}$} & {$\left[\mathrm{Co}(1 \mathrm{~b})(\mathrm{MeCN})_{2}\right](\mathrm{OTf})_{3}$} \\
\hline Appearance & purple plate & red block & orange block \\
\hline Formula & $\mathrm{C}_{45} \mathrm{H}_{54} \mathrm{CoF}_{6} \mathrm{~N}_{12} \mathrm{O}_{6} \mathrm{~S}_{2}$ & $\mathrm{C}_{37} \mathrm{H}_{32} \mathrm{ClCoF}_{15} \mathrm{~N}_{11} \mathrm{O}_{6} \mathrm{~S}_{2}$ & $\mathrm{C}_{40} \mathrm{H}_{33} \mathrm{CoF}_{18} \mathrm{~N}_{12} \mathrm{O}_{9} \mathrm{~S}_{3}$ \\
\hline$F W, \mathrm{~g} \mathrm{~mol}^{-1}$ & 1096.05 & 1170.23 & 1322.89 \\
\hline Cryst. Syst. & trigonal & tetragonal & orthorhombic \\
\hline Space group & P 3 c 1 & I 41/a & $\mathrm{Pbca}$ \\
\hline Size $\left(\mathrm{mm}^{3}\right)$ & $0.040 \times 0.240 \times 0.300$ & $0.160 \times 0.200 \times 0.220$ & $0.120 \times 0.220 \times 0.280$ \\
\hline$T, \mathrm{~K}$ & $100(2)$ & $100(2)$ & $100(2)$ \\
\hline Wavelength, $\AA$ & 0.71073 & 0.71073 & 1.54178 \\
\hline$a, \AA$ & $19.2173(4)$ & $26.8073(10)$ & $18.5579(10)$ \\
\hline$b, \AA$ & $19.2173(4)$ & $26.8073(10)$ & $21.1947(10)$ \\
\hline$c, \AA$ & $24.2408(6)$ & $26.8860(16)$ & $26.1256(12)$ \\
\hline$\alpha$, deg & 90 & 90 & 90 \\
\hline$\beta, \operatorname{deg}$ & 90 & 90 & 90 \\
\hline$\gamma$, deg & 120 & 90 & 90 \\
\hline$V, \AA^{3}$ & $7752.9(4)$ & 19321.1(18) & $10276.0(9)$ \\
\hline$Z$ & 6 & 16 & 8 \\
\hline$d_{\text {calc., }} \mathrm{g} \mathrm{cm}^{-3}$ & 1.409 & 1.609 & 1.710 \\
\hline$\mu, \mathrm{mm}^{-1}$ & 0.493 & 0.611 & 4.959 \\
\hline Refl. collected & 95488 & 124999 & 35018 \\
\hline GOF & 1.046 & 1.062 & 1.133 \\
\hline$\Delta / \sigma_{\max }$ & 0.005 & 0.001 & 0.001 \\
\hline$R ; \mathrm{I}>2 \sigma(\mathrm{I})$ & 0.0484 & 0.1114 & 0.1155 \\
\hline $\begin{array}{l}\text { Largest diff. peak } \\
\text { and hole, } \AA^{-3}\end{array}$ & $0.491 /-0.618$ & $1.724 /-1.071$ & $0.992 /-0.646$ \\
\hline $\begin{array}{l}\text { R.M.S. deviation } \\
\text { from mean, } \AA^{-3}\end{array}$ & 0.063 & 0.125 & 0.129 \\
\hline
\end{tabular}


Table S4. Selected bond lengths $(\AA)$ and angles $\left(^{\circ}\right)$ for $\left[\mathrm{Ag}_{3}(\mathbf{1} \mathbf{a})_{2}\right](\mathrm{OTf})_{3},\left[\mathrm{Ag}_{3}(\mathbf{1} \mathbf{b})_{2}\right]\left(\mathrm{BF}_{4}\right)_{3},\left[\mathrm{Ag}_{3}(\mathbf{1} \mathbf{c})_{2}\right]\left(\mathrm{BF}_{4}\right)_{3}$, and $\left[\mathrm{Ag}_{3}(\mathbf{1 d})_{2}\right](\mathrm{OTf})_{3} .{ }^{\mathrm{a}]}$

\begin{tabular}{|c|c|c|c|c|}
\hline & {$\left[\mathrm{Ag}_{3}(1 \mathrm{a})_{2}\right](\mathrm{OTf})_{3}$} & {$\left[\mathrm{Ag}_{3}(1 \mathrm{~b})_{2}\right]\left(\mathrm{BF}_{4}\right)_{3}$} & {$\left[\mathrm{Ag}_{3}(1 \mathrm{C})_{2}\right](\mathrm{BF} 4)_{3}$} & {$\left[\mathrm{Ag}_{3}(\mathbf{1 d})_{2}\right](\mathrm{OTf})_{3}$} \\
\hline $\mathrm{Ag}-\mathrm{C}$ & $\begin{array}{l}2.073(5), 2.078(5), 2.078(5), \\
2.084(5), 2.087(5), 2.100(7)\end{array}$ & $\begin{array}{l}\text { 2.120(7), 2.123(7), 2.117(7), } \\
2.112(6), 2.104(7), 2.100(7)\end{array}$ & $\begin{array}{c}2.085(9), 2.070(9), 2.070(9) \\
2.085(9), 2.07,2.07\end{array}$ & $\begin{array}{l}2.084(4), 2.084(4), 2.080(4), \\
2.082(4), 2.074(4), 2.072(4)\end{array}$ \\
\hline $\mathrm{C}-\mathrm{C}$ & $\begin{array}{c}5.953(7), 6.10(1), 5.80(1) \\
5.960(8) 5.924(7), 6.061(7)\end{array}$ & $\begin{array}{l}4.71(1), 4.24(1), 4.51(1), \\
4.33(1), 4.48(1), 4.80(1)\end{array}$ & $\begin{array}{c}5.94(1), 5.85(2), 5.98(1), \\
5.98(1), 5.94(1), 5.85(2)\end{array}$ & $\begin{array}{r}5.850(5), 5.985(6), 6.120(5), \\
6.163(5) 5.898(6), 5.862(5)\end{array}$ \\
\hline $\mathrm{Ag}-\mathrm{Ag}$ & $\begin{array}{c}5.9673(7), 6.0234(7) \\
6.0944(7)\end{array}$ & $3.184(1), 3.163(1), 2.983(1)$ & $5.994,5.994,5.793(1)$ & $\begin{array}{c}6.2809(6), 5.9293(6) \\
5.9876(6)\end{array}$ \\
\hline $\mathrm{C}_{\text {cent }}-\mathrm{Ag}_{\mathrm{cent}}$ & $2.085,2.078$ & $1.405,1.367$ & $2.060,2.375$ & $2.060,2.054$ \\
\hline $\mathrm{C}-\mathrm{Ag}-\mathrm{C}$ & $172.6(3), 177.5(2), 177.9(2)$ & $164.6(3), 160.3(3), 168.3(3)$ & $179.2,176.9(4), 176.9(4)$ & $174.9(2), 176.2(2), 176.6(2)$ \\
\hline $\mathrm{C}_{\text {cent }}-\mathrm{Ag}_{\text {cent }}-\mathrm{C}_{\text {cent }}$ & 177.38 & 175.40 & 170.68 & 178.89 \\
\hline $\begin{array}{l}\text { Angle between the mean } \\
\text { planes of the } 2 \mathrm{Trz} \text { rings } \\
\text { coordinated to each } \mathrm{Ag}^{+}\end{array}$ & $34.04,36.34,43.83$ & $84.69,89.86,52.85$ & $27.60,27.60,23.77$ & $30.48,28.96,27.28$ \\
\hline $\mathrm{C}-\mathrm{C}_{\text {cent }}-\mathrm{Ag}_{\text {cent }}-\mathrm{Ag}$ & $\begin{array}{c}-1.19,-1.13,-0.14,-1.18,- \\
1.41,1.26\end{array}$ & $\begin{array}{c}-36.27,-32.49,-39.31,-34.59 \\
-37.03,-41.87\end{array}$ & $\begin{array}{c}4.09,3.90,4.88,1.95,9.30 \\
1.30\end{array}$ & $\begin{array}{c}-4.66,-4.67,-4.29,-5.63,- \\
5.65,-4.37\end{array}$ \\
\hline
\end{tabular}

[a] $\mathrm{Ag}_{\mathrm{cent}}$ and $\mathrm{C}_{\text {cent }}$ correspond to the centroids of the silver(I) ions and coordinated $\mathrm{C}$-atoms of each ligand, respectively. 
Table S5. Selected bond lengths $(\AA ̊)$ and angles $\left(^{\circ}\right)$ for $[\mathrm{Co}(\mathbf{1 a})(\mathrm{MeCN})](\mathrm{OTf})_{2},[\mathrm{Co}(\mathbf{1 b})(\mathrm{Cl})(\mathrm{MeCN})](\mathrm{OTf})_{2}$, and $\left[\mathrm{Co}(\mathbf{1 b})(\mathrm{MeCN})_{2}\right](\mathrm{OTf})_{3}{ }^{[\mathrm{a}]}$

\begin{tabular}{cccc}
\hline Bond Length $(\AA)$ & {$[\mathrm{Co}(\mathbf{1 a})(\mathrm{MeCN})]^{2+}$} & {$[\mathrm{Co}(\mathbf{1 b})(\mathrm{Cl})(\mathrm{MeCN})]^{2+}$} & {$\left[\mathrm{Co}(\mathbf{1 b})(\mathrm{MeCN})_{2}\right]^{3+}$} \\
\hline Co1-C3 & $2.016(4)$ & $1.979(7)$ & $1.913(10)$ \\
Co1-C14 & $2.032(4)$ & $1.871(8)$ & $1.870(9)$ \\
Co1-C25 & $2.002(5)$ & $1.985(8)$ & $1.966(10)$ \\
Co1-N1 & 2.747 & $2.094(7)$ & $2.116(7)$ \\
Co1-N2 & $2.066(6)$ & $1.890(8)$ & $1.864(7)$ \\
Co1-N3 & - & - & $1.963(8)$ \\
Co1-Cl1 & - & $2.305(2)$ & -
\end{tabular}

\begin{tabular}{|c|c|c|c|}
\hline Bond Angle $\left({ }^{\circ}\right)$ & & & \\
\hline C3-Co1-C14 & $110.04(11)$ & $89.7(3)$ & $92.8(4)$ \\
\hline $\mathrm{C} 14-\mathrm{Co} 1-\mathrm{C} 25$ & 109.76(13) & $87.0(3)$ & $89.6(4)$ \\
\hline $\mathrm{C} 3-\mathrm{Co} 1-\mathrm{C} 25$ & $109.49(13)$ & $163.5(3)$ & $163.5(3)$ \\
\hline $\mathrm{C} 3-\mathrm{Co} 1-\mathrm{N} 1$ & - & $82.2(3)$ & $81.8(3)$ \\
\hline $\mathrm{C} 14-\mathrm{Co} 1-\mathrm{N} 1$ & - & $84.3(3)$ & $83.9(3)$ \\
\hline $\mathrm{C} 25-\mathrm{Co} 1-\mathrm{N} 1$ & - & $81.3(3)$ & $82.2(3)$ \\
\hline $\mathrm{C} 3-\mathrm{Co} 1-\mathrm{N} 2$ & $108.89(12)$ & $100.0(8)$ & $94.8(3)$ \\
\hline $\mathrm{C} 14-\mathrm{Co} 1-\mathrm{N} 2$ & 109.18(13) & $98.9(8)$ & $93.4(3)$ \\
\hline $\mathrm{C} 25-\mathrm{Co} 1-\mathrm{N} 2$ & $109.45(13)$ & $96.5(8)$ & $101.4(3)$ \\
\hline $\mathrm{C} 3-\mathrm{CO} 1-\mathrm{N} 3$ & - & - & $89.4(4)$ \\
\hline $\mathrm{C} 14-\mathrm{Co} 1-\mathrm{N} 3$ & - & - & $174.9(3)$ \\
\hline $\mathrm{C} 25-\mathrm{Co} 1-\mathrm{N} 3$ & - & - & $87.0(4)$ \\
\hline $\mathrm{C} 3-\mathrm{Co} 1-\mathrm{Cl} 1$ & - & $88.0(2)$ & - \\
\hline $\mathrm{C} 14-\mathrm{Co} 1-\mathrm{Cl} 1$ & - & $174.0(3)$ & - \\
\hline $\mathrm{C} 25-\mathrm{Co} 1-\mathrm{Cl} 1$ & - & $93.6(2)$ & - \\
\hline $\mathrm{N} 1-\mathrm{Co} 1-\mathrm{N} 2$ & - & $176.0(9)$ & $175.5(3)$ \\
\hline $\mathrm{N} 2-\mathrm{Co} 1-\mathrm{N} 3$ & - & - & $91.1(3)$ \\
\hline $\mathrm{N} 3-\mathrm{Co} 1-\mathrm{N} 1$ & - & - & $91.8(3)$ \\
\hline $\mathrm{N} 1-\mathrm{Co} 1-\mathrm{Cl}$ & - & $89.9(2)$ & - \\
\hline $\mathrm{N} 2-\mathrm{Co} 1-\mathrm{Cl} 1$ & - & $86.9(8)$ & - \\
\hline $\begin{array}{l}\text { Angle between the Trz } \\
\text { rings and aryl rings }\end{array}$ & $81.78,87.50,87.96$ & $49.34,50.84,76.05$ & $47.29,78.88,86.82$ \\
\hline
\end{tabular}

[a] The X-ray crystal structure of $[\mathrm{Co}(1 \mathrm{a})(\mathrm{MeCN})](\mathrm{OTf})_{2}$ contains 3 molecules per unit cell. The structural parameters of all three are similar, so those of only one is listed. 


\section{Density Functional Theory (DFT) Calculations}

DFT calculations were performed with Gaussian 16, ${ }^{[13]}$ using the Becke Three-Parameter functional with the non-local correlation by Perdew and Wang (B3PW91) ${ }^{[14]}$ and the D3 version of Grimme's dispersion with Becke-Johnson damping (GD3BJ) ${ }^{[15]}$. Whereas $\mathrm{H}, \mathrm{C}$ and $\mathrm{N}$ atoms were represented with the 6-31G(d,p)++ basis set, $\mathrm{Ag}$ atoms were described by the Stuttgart/Dresden Effective Core Potential and the associated basis set, as implemented in Gaussian 16a. ${ }^{[16]}$ Molecular geometries of the methylated derivatives of 3a were optimized without any geometric constraints in both vacuum and using the SMD continuum solvent model (dichloromethane) ${ }^{[17]}$, whereas geometry optimizations of the "open" and "closed" structures of $\left[\mathrm{Ag}_{3}(\mathbf{1} \mathbf{a})_{2}\right]^{3+}$ and $\left[\mathrm{Ag}_{3}(\mathbf{1} \mathbf{b})_{2}\right]^{3+}$ were performed only in vacuum. To confirm that the optimized structures correspond to energetic minima and calculate free energy $(G)$ corrections, frequency calculations were performed at the same level of theory. Calculations of QTAIM (quantum theory of atoms in molecules) plots were performed using Multiwfn ${ }^{[18]}$ software and the bond nature of the critical points $(3,-1)$ were checked manually in the log file. A list of coordinates for computed minima can be found below.

Table S6. DFT calculated free energies of mono-, di- and trimethylated $3 a$ in both vacuum and dichloromethane. The latter are given in parentheses.

\begin{tabular}{ccccccc}
\hline Me position & \multicolumn{2}{c}{ Monomethylated } & \multicolumn{2}{c}{ Dimethylated } & \multicolumn{2}{c}{ Trimethylated } \\
& $\mathrm{G}($ a.u. $)$ & $\begin{array}{c}\Delta \mathrm{G}^{\mathrm{a}} \\
(\mathrm{kcal} / \mathrm{mol})\end{array}$ & $\mathrm{G}($ a.u. $)$ & $\begin{array}{c}\Delta \mathrm{G}^{\mathrm{a}} \\
(\mathrm{kcal} / \mathrm{mol})\end{array}$ & $\mathrm{G}($ a.u. $)$ & $\begin{array}{c}\Delta \mathrm{G}^{\mathrm{a}} \\
(\mathrm{kcal} / \mathrm{mol})\end{array}$ \\
\hline Triazole & -1983.12 & 0.00 & -2022.71 & 0.00 & -2062.19 & 0.00 \\
& $(-1983.22)$ & $(0.00)$ & $(-2022.92)$ & $(0.00)$ & $(-2062.61)$ & $(0.00)$ \\
Central amine (+ & -1983.10 & 11.7 & -2022.69 & 13.5 & -2062.15 & 26.1 \\
Triazole) & $(-1983.21)$ & $(2.69)$ & $(-2022.91)$ & $(2.97)$ & $(-2062.59)$ & $(11.7)$ \\
\hline
\end{tabular}

a Free energies are given relative to the lowest energy isomer.

Table S7. DFT calculated free energies (in vacuum) of "open" and "closed" structures of $\left[\operatorname{Ag}_{3}(\mathbf{1 a})_{2}\right]^{3+}$ and $\left[\mathrm{Ag}_{3}(\mathbf{1} \mathbf{b})_{2}\right]^{3+}$. ${ }^{\mathrm{a}}$ Free energies $(\Delta \mathrm{G})$ are given relative to the lowest energy isomer.

\begin{tabular}{ccccc}
\hline Structure & \multicolumn{2}{c}{$\left[\mathrm{Ag}_{3}(\mathbf{1 b})_{2}\right]^{3+}$} & \multicolumn{2}{c}{$\left[\mathrm{Ag}_{3}(\mathbf{1 a})_{2}\right]^{3+}$} \\
& $\mathrm{G}(\mathrm{a} . \mathrm{u})$ & $\begin{array}{c}\Delta \mathrm{G}^{\mathrm{a}} \\
(\mathrm{kcal} / \mathrm{mol})\end{array}$ & $\mathrm{G}(\mathrm{a} . \mathrm{u})$ & $\begin{array}{c}\Delta \mathrm{G}^{\mathrm{a}} \\
(\mathrm{kcal} / \mathrm{mol})\end{array}$ \\
\hline Open & 5877.37 & 31.84 & 4563.10 & 0.00 \\
Closed & 5877.42 & 0.00 & 4563.06 & 22.38 \\
\hline
\end{tabular}




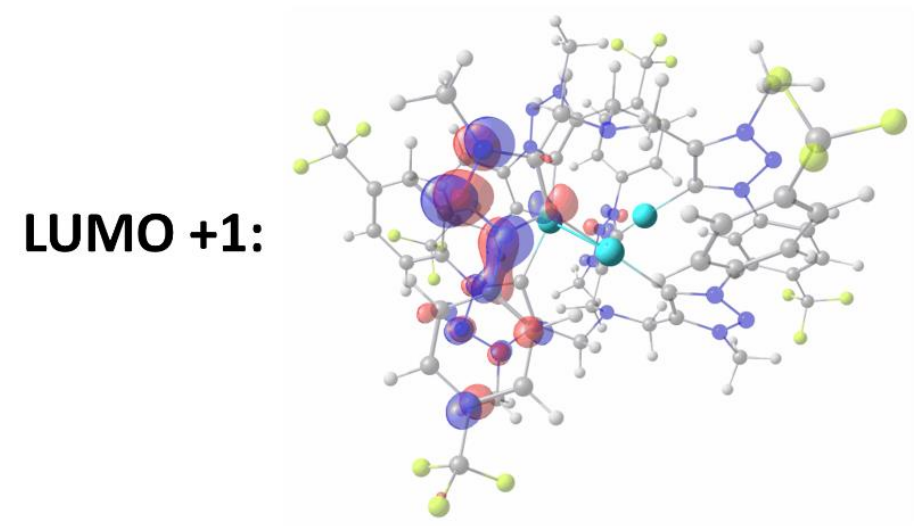

$-8.49 \mathrm{eV}$

LUMO:

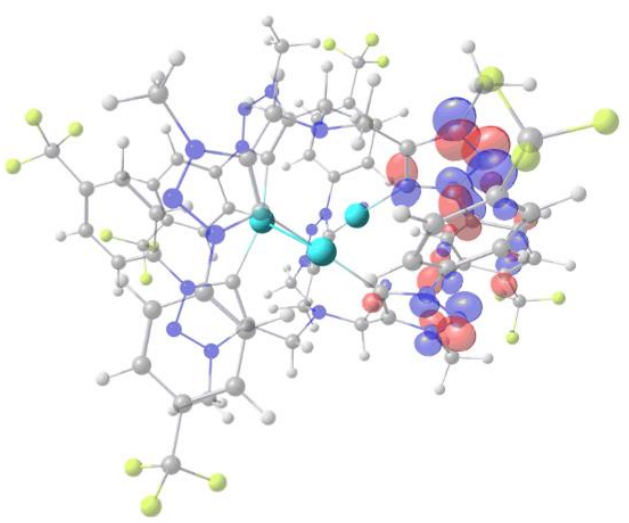

$-8.54 \mathrm{eV}$

HOMO:

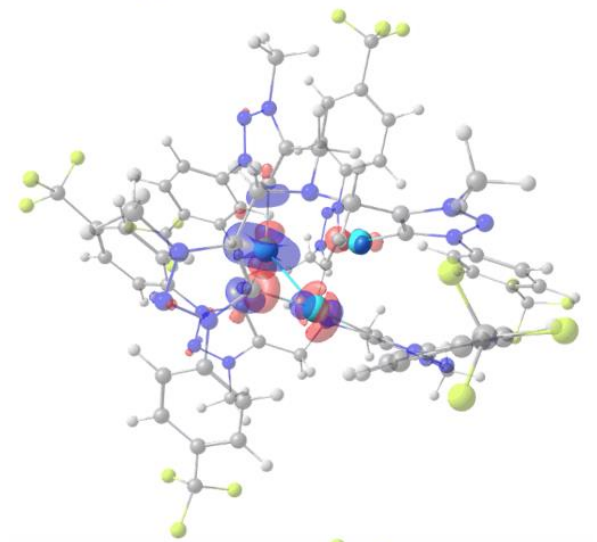

$-12.54 \mathrm{eV}$

HOMO -1:

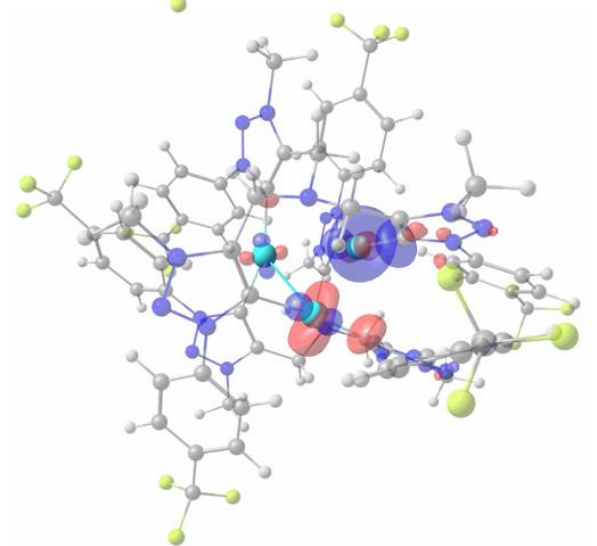

$-12.66 \mathrm{eV}$

Figure S112. Frontier orbital plots for the "closed" structure of $\left[\operatorname{Ag}_{3}(1 \mathbf{a})_{2}\right]^{3+}$. 


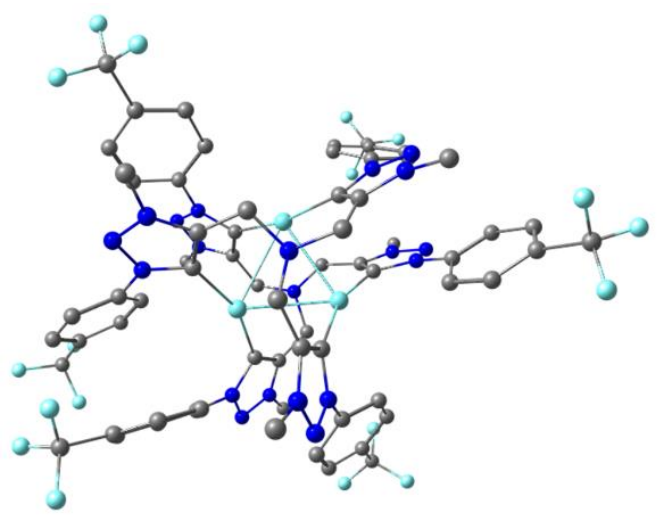

$0.0 \mathrm{kcal} / \mathrm{mol}$

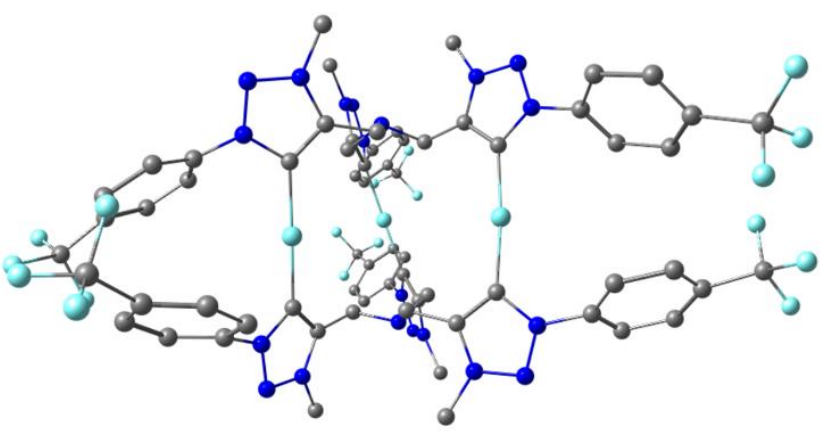

$31.8 \mathrm{kcal} / \mathrm{mol}$

Figure S113. DFT geometry optimized "closed" (left) and "open" (right) structures of $\left[\mathrm{Ag}_{3}(\mathbf{1} \mathbf{b})_{2}\right]^{3+}$.

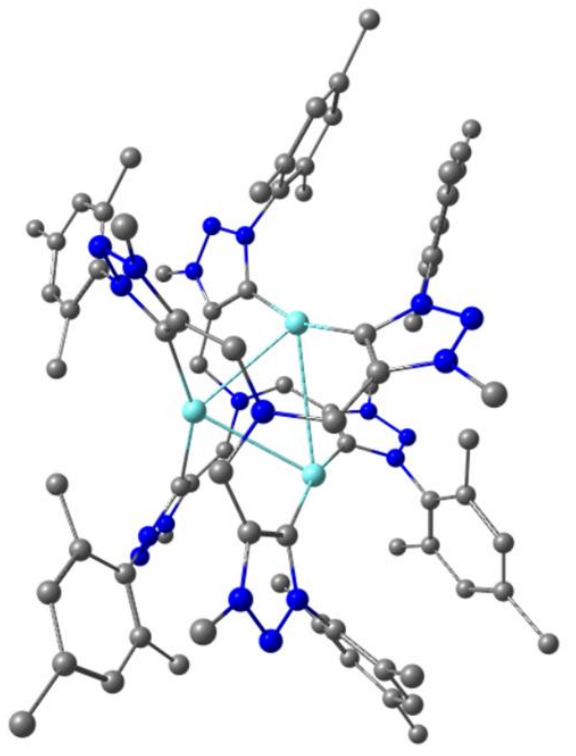

$22.3 \mathrm{kcal} / \mathrm{mol}$

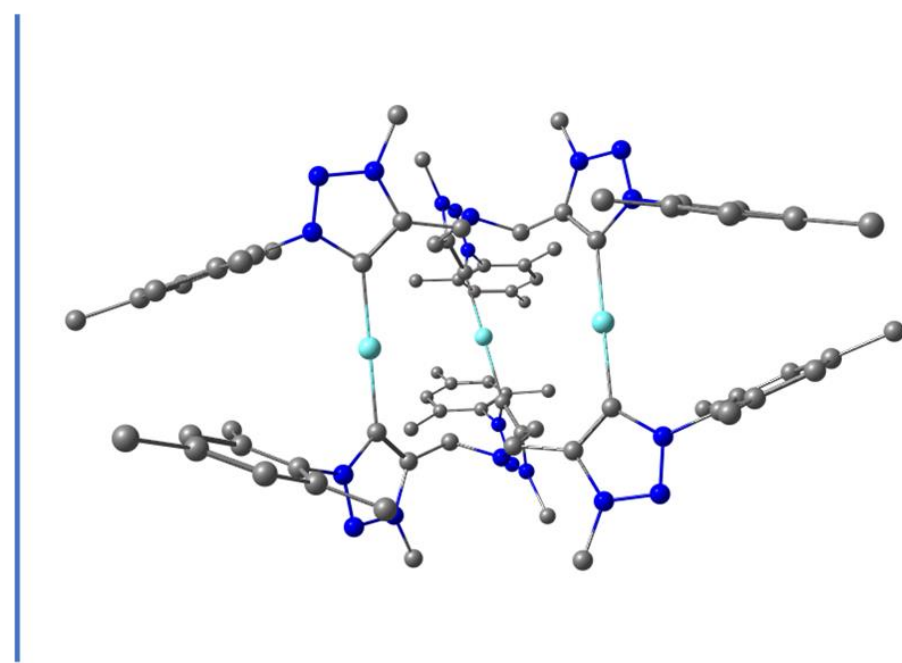

$0.0 \mathrm{kcal} / \mathrm{mol}$

Figure S114. DFT geometry optimized "closed" (left) and "open" (right) structures of $\left[\operatorname{Ag}_{3}(1 \mathbf{a})_{2}\right]^{3+}$. 


\section{Atomic coordinates of DFT geometry optimized structures}

\begin{tabular}{|c|c|c|c|}
\hline \multicolumn{4}{|c|}{ onomethylated 3a (triazole) } \\
\hline & -2.201607000 & -1.576569000 & -2.05062000 \\
\hline & -1.954273000 & -2.630478000 & -2.20 \\
\hline & -2.244859000 & -1.092202000 & -3.030246000 \\
\hline & & -1.490330000 & \\
\hline & -4.489049000 & -0.493787000 & -1.388672000 \\
\hline & 4861000 & 0.426694000 & -1.943343000 \\
\hline & -6.606601000 & -0.216204000 & -0.085553000 \\
\hline & & & \\
\hline & & 000 & 000 \\
\hline & & 000 & 000 \\
\hline & 6000 & 1.735294000 & 1000 \\
\hline & & & \\
\hline & & & \\
\hline & & & \\
\hline & 000 & 5000 & 6000 \\
\hline & & & \\
\hline & 988000 & 6000 & 6000 \\
\hline & & & \\
\hline & & & 5000 \\
\hline & 7000 & 000 & 0000 \\
\hline & & & 000 \\
\hline & & & \\
\hline & & & \\
\hline & & & 7000 \\
\hline & & & \\
\hline & & & 000 \\
\hline & & & \\
\hline & & & \\
\hline & -1 & & 000 \\
\hline & & & 00 \\
\hline & & & 000 \\
\hline & & & 000 \\
\hline & & & \\
\hline & & & 00 \\
\hline & & & \\
\hline & & & \\
\hline & & & \\
\hline & & & 000 \\
\hline & & & \\
\hline & & & \\
\hline & & -3. & 000 \\
\hline & 8.072722000 & 3000 & -0.8 \\
\hline & & & \\
\hline & & & \\
\hline & & & 3000 \\
\hline & & & 2000 \\
\hline & & -1.2 & 1000 \\
\hline & & -1.5 & $-0 . \varepsilon$ \\
\hline & 4.106643000 & -0.3 & -1. \\
\hline & 3.600111000 & -0.621385000 & -2.404972000 \\
\hline & 4.874307000 & 0.389928000 & -1.717257000 \\
\hline & & & \\
\hline & -1.255911000 & 0.49 & -1.23 \\
\hline 1 & -2.178600000 & 0.772526000 & -0.694725000 \\
\hline & -1.345182000 & 0.867120000 & -2.256427000 \\
\hline & & & -0 \\
\hline & 1.066452000 & 1.668549000 & -1.044290000 \\
\hline & 1.511380000 & 1.647471000 & -2.026409000 \\
\hline 6 & 2.924898000 & 2.937843000 & 0.050103000 \\
\hline
\end{tabular}

$\begin{array}{rrrr}6 & 3.092703000 & 4.065077000 & -0.769664000 \\ 6 & 1.985977000 & 4.593412000 & -1.638715000 \\ 1 & 1.018109000 & 4.551006000 & -1.130252000 \\ 1 & 1.891736000 & 4.022670000 & -2.569501000 \\ 1 & 2.185364000 & 5.632684000 & -1.910701000 \\ 6 & 4.334343000 & 4.701064000 & -0.747808000 \\ 1 & 4.482746000 & 5.575960000 & -1.376135000 \\ 6 & 5.381397000 & 4.252545000 & 0.062612000 \\ 6 & 6.698299000 & 4.975604000 & 0.084201000 \\ 1 & 6.946248000 & 5.384200000 & -0.899884000 \\ 1 & 7.510704000 & 4.315435000 & 0.400668000 \\ 1 & 6.665974000 & 5.817216000 & 0.787141000 \\ 6 & 5.163858000 & 3.137360000 & 0.873737000 \\ 1 & 5.967082000 & 2.774618000 & 1.510633000 \\ 6 & 3.941022000 & 2.463195000 & 0.891125000 \\ 6 & 3.746746000 & 1.277255000 & 1.788764000 \\ 1 & 3.353610000 & 1.585315000 & 2.763870000 \\ 1 & 4.695504000 & 0.761470000 & 1.956247000 \\ 1 & 3.034126000 & 0.565268000 & 1.373458000 \\ 7 & -1.097623000 & -0.959444000 & -1.301101000 \\ 7 & -3.938289000 & -2.434846000 & -0.472212000 \\ 7 & -5.074569000 & -2.078637000 & 0.057088000 \\ 7 & -5.420336000 & -0.898555000 & -0.494558000 \\ 7 & 2.353452000 & -1.730903000 & 1.627328000 \\ 7 & 2.666729000 & -2.044082000 & 0.385542000 \\ 7 & -0.173232000 & 1.498924000 & 0.782673000 \\ 7 & 0.910687000 & 2.136168000 & 1.124469000 \\ 7 & 1.675268000 & 2.247999000 & 0.018917000 \\ 7 & 1.057535000 & -1.512833000 & 1.594484000 \\ 6 & 0.372519000 & -1.118317000 & 2.817162000 \\ 1 & -0.336213000 & -1.897455000 & 3.100817000 \\ 1 & 1.129393000 & -0.993343000 & 3.589248000 \\ 1 & -0.143691000 & -0.174714000 & 2.631189000\end{array}$

Monomethylated 3a (central amine)

$\begin{array}{lrrr}6 & -0.023031000 & -1.398108000 & -0.172421000 \\ 1 & 0.778811000 & -1.900159000 & 0.368001000 \\ 1 & -0.974369000 & -1.760423000 & 0.219731000 \\ 6 & 0.155323000 & 0.060937000 & 0.023307000 \\ 6 & -0.448871000 & 1.182027000 & -0.505328000 \\ 1 & -1.220528000 & 1.341355000 & -1.241066000 \\ 6 & -0.031917000 & 3.615137000 & -0.087095000 \\ 6 & 0.982667000 & 4.353973000 & -0.710109000 \\ 6 & 2.262864000 & 3.707303000 & -1.153706000 \\ 1 & 2.810897000 & 4.367962000 & -1.829792000 \\ 1 & 2.088192000 & 2.753180000 & -1.659736000 \\ 1 & 2.908391000 & 3.486791000 & -0.296946000 \\ 6 & 0.757463000 & 5.719060000 & -0.895561000 \\ 1 & 1.526837000 & 6.313838000 & -1.381967000 \\ 6 & -0.425265000 & 6.336096000 & -0.477119000 \\ 6 & -0.624552000 & 7.814030000 & -0.662496000 \\ 1 & -1.684344000 & 8.065126000 & -0.762972000 \\ 1 & -0.095410000 & 8.180111000 & -1.547017000 \\ 1 & -0.237438000 & 8.367296000 & 0.202104000 \\ 6 & -1.405561000 & 5.556901000 & 0.144191000 \\ 1 & -2.327701000 & 6.024864000 & 0.480183000 \\ 6 & -1.230089000 & 4.188428000 & 0.356250000\end{array}$




\begin{tabular}{|c|c|c|c|}
\hline & -2.289490000 & 3.366189000 & 3000 \\
\hline & -3.002359000 & 4.012637000 & 1.551126000 \\
\hline & -1.855513000 & 00 & 000 \\
\hline & -2.853499000 & .764937000 & .311431000 \\
\hline & 1.201525000 & -1.317561000 & 38000 \\
\hline & 0.865355000 & -0.363779000 & -2.805713000 \\
\hline & 1.354974000 & -2.013209000 & -3.227027000 \\
\hline & 2.443306000 & -1.038942000 & -1.637328000 \\
\hline & 3.314024000 & -1.821973000 & -0.909274000 \\
\hline & 3.344580000 & -2.872412000 & -0.666058000 \\
\hline & 5.395301000 & -1.2226 & 000 \\
\hline & 5.429020000 & -0.688 & 7000 \\
\hline & 4.308762000 & 5000 & 6000 \\
\hline & 4.340004000 & 1.164861000 & 1.744576000 \\
\hline & 4.382930000 & 0.251830000 & 2000 \\
\hline & 3.326 & -0.251 & 3000 \\
\hline & 6.556178000 & -0.961484000 & 2.419993000 \\
\hline & 6.605028000 & -0.558689000 & 3.428836000 \\
\hline & 7.616 & -1.73 & 000 \\
\hline & 8.80 & -2.0 & 000 \\
\hline & 8.585545000 & -2.872814000 & 3.484325000 \\
\hline & 9.063536000 & -1.175810000 & 07000 \\
\hline & 9.677629000 & -2.3 & 2.2 \\
\hline & 7.541422000 & -2.235831000 & 0.635 \\
\hline & 8.365648000 & -2.824413000 & 0.239927000 \\
\hline & 6.438331000 & -1.99 & 1000 \\
\hline & 6.386941000 & -2.52204 & -1.5 \\
\hline & 7.376959000 & -2.859718000 & 46000 \\
\hline & 6.042098000 & -1.758600000 & -2.293655000 \\
\hline & 5.703514000 & -3.374754000 & -1.672451000 \\
\hline & -1.22 & -1. & 9000 \\
\hline & -1.122658000 & -2.030139000 & -3.34 \\
\hline & -1.198000000 & -0.455573000 & -2.530726000 \\
\hline & -2.477965000 & -1.916116000 & -1.691141000 \\
\hline & -3.317621000 & -1.125727000 & -0.932964000 \\
\hline & -3.299218000 & -0.081869000 & -0.661493000 \\
\hline & -5.408394000 & -1.676817000 & 0.314712000 \\
\hline & -6.687331000 & -1.641781000 & -0.254766000 \\
\hline & -6.894487000 & -1.907599000 & -1.717894000 \\
\hline & -6.693984000 & -2.956475000 & -1.960008000 \\
\hline & -6.220446000 & -1.302384000 & -2.333602000 \\
\hline & -7.922616000 & -1.680618000 & -2.008414000 \\
\hline & -7.757911000 & -1.356745000 & 0.593093000 \\
\hline & -8.761664000 & -1.324593000 & 0.176517000 \\
\hline & -7.572015000 & -1.111364000 & 1.957740000 \\
\hline & -8.742227000 & -0.777554000 & 2.839189000 \\
\hline & -8.997698000 & 0.286152000 & 2.757194000 \\
\hline & -8.521751000 & -0.983311000 & 3.890185000 \\
\hline & -9.631612000 & -1.347116000 & 2.553449000 \\
\hline & -6.276636000 & -1.163117000 & 2.479673000 \\
\hline & -6.119822000 & -0.987463000 & 3.541045000 \\
\hline & -5.171637000 & -1.447536000 & 1.675034000 \\
\hline & -3.785092000 & -1.514892000 & 2.249783000 \\
\hline & -3.256226000 & -2.409950000 & 1.906089000 \\
\hline & & $-1.537>$ & 3.341148 \\
\hline
\end{tabular}

$\begin{array}{lrrr}1 & -3.184110000 & -0.646217000 & 1.956842000 \\ 7 & 0.040389000 & -1.894447000 & -1.597765000 \\ 7 & 1.090530000 & 0.475317000 & 0.926297000 \\ 7 & 1.097704000 & 1.771417000 & 0.982144000 \\ 7 & 0.173490000 & 2.216078000 & 0.109360000 \\ 7 & 3.999116000 & 0.295340000 & -0.907868000 \\ 7 & 4.254460000 & -0.954269000 & -0.468699000 \\ 7 & -2.989178000 & -3.181100000 & -1.710687000 \\ 7 & -4.079181000 & -3.213021000 & -1.004151000 \\ 7 & -4.291581000 & -1.966449000 & -0.527880000 \\ 7 & 2.906550000 & 0.245062000 & -1.603995000 \\ 6 & 0.173593000 & -3.383244000 & -1.526029000 \\ 1 & 1.125981000 & -3.620369000 & -1.052313000 \\ 1 & 0.136140000 & -3.786426000 & -2.537188000 \\ 1 & -0.649219000 & -3.785793000 & -0.936668000\end{array}$

\section{Dimethylated 3a (triazole only).}

$\begin{array}{llll}6 & -0.200952000 & -0.537302000 & 0.102028000 \\ 1 & -1.107916000 & -0.861984000 & 0.618856000 \\ 1 & 0.103257000 & 0.406253000 & 0.560275000 \\ 6 & 0.878429000 & -1.549946000 & 0.297650000 \\ 6 & 2.234880000 & -1.365282000 & 0.469134000 \\ 1 & 2.849872000 & -0.483114000 & 0.560252000 \\ 6 & 4.115468000 & -3.005207000 & 0.646317000 \\ 6 & 4.804792000 & -3.409606000 & -0.502747000 \\ 6 & 4.120974000 & -3.463088000 & -1.838566000 \\ 1 & 4.849878000 & -3.596696000 & -2.641099000 \\ 1 & 3.551977000 & -2.548477000 & -2.037618000 \\ 1 & 3.410740000 & -4.295897000 & -1.880816000 \\ 6 & 6.144978000 & -3.770600000 & -0.355020000 \\ 1 & 6.703346000 & -4.083894000 & -1.233745000 \\ 6 & 6.781328000 & -3.739173000 & 0.889592000 \\ 6 & 8.215713000 & -4.165005000 & 1.027806000 \\ 1 & 8.719305000 & -3.618232000 & 1.830203000 \\ 1 & 8.770083000 & -4.007933000 & 0.098239000 \\ 1 & 8.279086000 & -5.232871000 & 1.270708000 \\ 6 & 6.049134000 & -3.331625000 & 2.009299000 \\ 1 & 6.531412000 & -3.306551000 & 2.983277000 \\ 6 & 4.707312000 & -2.960281000 & 1.913889000 \\ 6 & 3.924411000 & -2.529907000 & 3.120527000 \\ 1 & 4.489035000 & -2.726012000 & 4.034703000 \\ 1 & 2.969478000 & -3.061980000 & 3.182559000 \\ 1 & 3.695888000 & -1.458523000 & 3.089191000 \\ 6 & -1.243183000 & -1.410721000 & -1.924687000 \\ 1 & -0.645352000 & -2.333199000 & -1.903767000 \\ 1 & -1.421856000 & -1.166983000 & -2.977279000 \\ 6 & -2.558204000 & -1.651673000 & -1.267098000 \\ 6 & -3.577602000 & -0.781785000 & -0.954599000 \\ 1 & -3.686180000 & 0.280877000 & -1.102366000 \\ 6 & -5.797435000 & -1.103619000 & 0.162475000 \\ 6 & -5.870825000 & -0.780980000 & 1.520505000 \\ 6 & -4.672283000 & -0.895303000 & 2.416501000 \\ 1 & -4.304733000 & -1.926208000 & 2.459913000 \\ 1 & -4.922245000 & -0.581012000 & 3.431702000\end{array}$




\begin{tabular}{|c|c|c|c|}
\hline & 0 & & \\
\hline & 5000 & 5000 & \\
\hline & -7.204140000 & .103934000 & 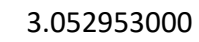 \\
\hline & -8.2280 & & \\
\hline & -9.5444 & 0 & \\
\hline & -9.662039000 & 1000 & \\
\hline & 178000 & 0.062725000 & \\
\hline & 9000 & 000 & \\
\hline & -8.09 & -0.6 & \\
\hline & -8.961261000 & -0.537 & \\
\hline & -6.8808 & & \\
\hline & -6.7 & & \\
\hline & -7.6 & 00 & \\
\hline & 000 & 00 & \\
\hline & -6. & 00 & \\
\hline & 0.5 & & \\
\hline & 0.213942000 & 00 & -3. \\
\hline & 1.345 & -0.6 & -2 \\
\hline & & & \\
\hline & 0.6 & & \\
\hline & -0.37 & 000 & \\
\hline & 000 & 00 & \\
\hline & 1.4 & & \\
\hline & 1.36 & 0 & \\
\hline & 2.16 & 00 & \\
\hline & 0.4 & & \\
\hline & & & \\
\hline & 1.43 & & \\
\hline & 1.31 & 00 & \\
\hline & 1.54 & 0 & \\
\hline & & & \\
\hline & 0.45 & & \\
\hline & 2.17 & & \\
\hline & 1.6 & 0 & \\
\hline & & & \\
\hline & 1.801 & 00 & -0.9 \\
\hline & 1.765670 & 723 & כ. \\
\hline & & & \\
\hline & & & \\
\hline & .828105000 & 6.584032000 & -2.91 \\
\hline & 1.24 & 00 & -2.8 \\
\hline & -0.562777000 & & \\
\hline & 0.648270000 & -2.894361000 & \\
\hline & 1.774550000 & -3.537609 & 037 \\
\hline & 0 & 0 & \\
\hline & & & \\
\hline & -4.530353000 & -1.538211000 & \\
\hline & 2.843181000 & 2.789231000 & -1.1 \\
\hline & 1.687841000 & 298862000 & -0.78 \\
\hline & -2.9930 & -2.86 & -0.8 \\
\hline & -2.294477000 & -4.1412 & \\
\hline & -2.203667000 & -4.4498680 & \\
\hline & -1.307461000 & -4.012222000 & -0.426835 \\
\hline & -2.88884 & -4.862906000 & 0.3 \\
\hline & & & \\
\hline
\end{tabular}

$\begin{array}{llll}6 & 3.600281000 & 0.792847000 & -2.219733000 \\ 1 & 3.525506000 & -0.215244000 & -1.810745000 \\ 1 & 3.503208000 & 0.765165000 & -3.305977000 \\ 1 & 4.547763000 & 1.246708000 & -1.935520000\end{array}$

Dimethylated 3a (central amine + triazole).

\begin{tabular}{|c|c|c|c|}
\hline & 3000 & 6000 & 0 \\
\hline & -1.062982000 & -2.174187000 & -1.8139760 \\
\hline & 0 & 0 & \\
\hline & 000 & 0 & \\
\hline & -3.9 & & \\
\hline & -4.297427000 & .000 & -1 \\
\hline & -6.20 & 00 & \\
\hline & -6.4 & & \\
\hline & -5.3 & & 0 \\
\hline & -5.759128000 & 000 & 00 \\
\hline & -4.8 & & \\
\hline & & & \\
\hline & -7.8 & 00 & \\
\hline & -8.053526 & 00 & \\
\hline & -8.8 & & \\
\hline & 0 & & \\
\hline & 0.925699000 & 000 & - 11 \\
\hline & -10.3 & 000 & \\
\hline & -10. & & \\
\hline & & & \\
\hline & 00 & & \\
\hline & 00 & 00 & \\
\hline & 0 & & \\
\hline & 0 & & \\
\hline & -6.3 & -3. & \\
\hline & 0 & & \\
\hline & 0.0 & & \\
\hline & 0 & & \\
\hline & 0 & & \\
\hline & .9774 & 0 & $37+3$ \\
\hline & 0 & & \\
\hline & & & \\
\hline & 26124 & & \\
\hline & 0 & & \\
\hline & & & \\
\hline & & & \\
\hline & .830756000 & -3.530709000 & -3.09 \\
\hline & $3.72880 €$ & 0 & \\
\hline & & & \\
\hline & 6.774898000 & -4.099085000 & -1.87 \\
\hline & 6.93233 & 00 & \\
\hline & & & \\
\hline & 0 & & \\
\hline & 8.661966000 & -5.120708000 & -0.620023 \\
\hline & 8.6693 & -5.12 & 1565308 \\
\hline & 0 & 0 & 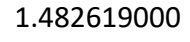 \\
\hline & 0 & -4.22 & 202 \\
\hline & 4.889681000 & -3.707341000 & \\
\hline
\end{tabular}




\begin{tabular}{lrrr}
6 & 4.140923000 & -3.565347000 & 2.811428000 \\
1 & 4.788741000 & -3.804012000 & 3.656957000 \\
1 & 3.275179000 & -4.235664000 & 2.844951000 \\
1 & 3.768085000 & -2.543863000 & 2.946416000 \\
6 & -1.089499000 & 0.994521000 & -0.553055000 \\
1 & -1.846515000 & 0.806145000 & 0.209013000 \\
1 & -1.576838000 & 1.340323000 & -1.465316000 \\
6 & -0.089432000 & 1.984296000 & -0.084142000 \\
6 & 0.455074000 & 3.068195000 & -0.742364000 \\
1 & 0.307078000 & 3.484933000 & -1.726721000 \\
6 & 2.143771000 & 4.760309000 & 0.010526000 \\
6 & 1.710673000 & 5.971033000 & 0.563250000 \\
6 & 0.394515000 & 6.067923000 & 1.278405000 \\
1 & 0.388399000 & 5.444966000 & 2.179165000 \\
1 & -0.429737000 & 5.723900000 & 0.644009000 \\
1 & 0.191449000 & 7.099405000 & 1.573854000 \\
6 & 2.555912000 & 7.073077000 & 0.430695000 \\
1 & 2.245122000 & 8.024762000 & 0.854637000 \\
6 & 3.787913000 & 6.980825000 & -0.225482000 \\
6 & 4.665451000 & 8.191263000 & -0.374695000 \\
1 & 4.369911000 & 8.777152000 & -1.253810000 \\
1 & 5.714210000 & 7.910571000 & -0.504673000 \\
1 & 4.587743000 & 8.849719000 & 0.495303000 \\
6 & 4.177840000 & 5.747219000 & -0.755527000 \\
1 & 5.138305000 & 5.659473000 & -1.256937000 \\
1 & -0.334942000 & -5.018419000 & 0.905685000 \\
6 & 3.368688000 & 4.614849000 & -0.648852000 \\
6 & 3.794663000 & 3.288250000 & -1.208967000 \\
1 & 3.706569000 & 2.495329000 & -0.458169000 \\
1 & 4.832319000 & 3.327440000 & -1.547550000 \\
1 & 3.172693000 & 2.999597000 & -2.064228000 \\
7 & -0.478551000 & -0.366092000 & -0.889663000 \\
7 & -3.010438000 & -2.135811000 & 0.127224000 \\
7 & -4.230188000 & -2.012666000 & 0.557709000 \\
7 & -4.841348000 & -1.103483000 & -0.227719000 \\
7 & 1.940969000 & -4.067733000 & 0.222656000 \\
7 & 2.870245000 & -3.135432000 & 0.290847000 \\
7 & 0.460224000 & 1.930755000 & 1.163552000 \\
\hline & 1.307442000 & 2.905183000 & 1.305358000 \\
\hline & 1.307238000 & 3.608582000 & 0.154680000 \\
\hline & 0.686993000 & -0.183134000 & -1.803922000 \\
\hline & 1.010714000 & -1.161874000 & -2.155074000 \\
\hline
\end{tabular}

\section{Trimethylated 3a (triazole only).}

$\begin{array}{rrrr}6 & -0.714632000 & -0.380244000 & -0.294657000 \\ 1 & -0.080256000 & -1.091393000 & -0.829232000 \\ 1 & -0.567609000 & 0.589126000 & -0.776098000 \\ 6 & -2.156046000 & -0.762513000 & -0.476925000\end{array}$

\begin{tabular}{|c|c|c|c|}
\hline & & & \\
\hline & 3.470273000 & 000 & 00 \\
\hline & 5.737659000 & 00 & -0.602334000 \\
\hline & 0 & 0 & \\
\hline & -5.8 & 00 & 000 \\
\hline & -6.4884 & 3000 & 3000 \\
\hline & 000 & 5000 & 2000 \\
\hline & & 00 & 000 \\
\hline & 00 & -0.6 & 000 \\
\hline & -8.415546000 & 393000 & 7000 \\
\hline & -8.4 & 0 & \\
\hline & -9.9 & & \\
\hline & -10 . & 00 & 00 \\
\hline & 000 & & \\
\hline & 00 & 00 & \\
\hline & 0 & 0.3 & \\
\hline & -8.122727000 & 0.82 & -2 \\
\hline & -6.29 & 0.0 & \\
\hline & -5.4 & & \\
\hline & -6. & & \\
\hline & 00 & 00 & boc \\
\hline & -4.7 & 1.1 & -2 \\
\hline & -0.2 & 0 & \\
\hline & 0 & 00 & \\
\hline & -0.1 & -1.3 & \\
\hline & 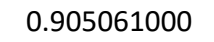 & -2.3 & \\
\hline & & & \\
\hline & 00 & & \\
\hline & 4.03 & -3.3 & -0 . \\
\hline & 4.0 & -3.8 & \\
\hline & & & \\
\hline & 0 & & \\
\hline & 2.9 & -4.3 & \\
\hline & U & -3 & \\
\hline & & & \\
\hline & 0 & -4.35 & -2. \\
\hline & .47 & -3.73 & -1 \\
\hline & & & \\
\hline & & & \\
\hline & .776606000 & -4.720162000 & -2.69 \\
\hline & 3.6 & -4.0 & \\
\hline & & -3.3 & \\
\hline & & -3.102 & \\
\hline & 1807 & -3.1192 & 00 \\
\hline & & -2.65 & \\
\hline & & -2.6 & \\
\hline & 4.473624000 & -3.29697 & \\
\hline & 4.723607000 & -1.633 & 00 \\
\hline & -0.80 & 0.8 & \\
\hline & -0.57 & 0 & \\
\hline & & 20 & \\
\hline & & & \\
\hline & 1.0272 & 2.34 & \\
\hline & 1.79 & 1.668317000 & 0.39 \\
\hline & & & \\
\hline
\end{tabular}




\begin{tabular}{llll}
6 & 2.287033000 & 4.475709000 & -1.383234000 \\
6 & 1.277789000 & 3.793079000 & -2.260537000 \\
1 & 0.255621000 & 4.067817000 & -1.978526000 \\
1 & 1.357309000 & 2.701905000 & -2.191272000 \\
1 & 1.430043000 & 4.072773000 & -3.304764000 \\
6 & 3.359037000 & 5.188981000 & -1.920237000 \\
1 & 3.460239000 & 5.243374000 & -3.000922000 \\
6 & 4.294864000 & 5.835813000 & -1.106479000 \\
6 & 5.421076000 & 6.622099000 & -1.713261000 \\
1 & 6.308324000 & 6.605865000 & -1.074182000 \\
1 & 5.129499000 & 7.671786000 & -1.840546000 \\
1 & 5.692458000 & 6.235301000 & -2.699227000 \\
6 & 4.147802000 & 5.762742000 & 0.282653000 \\
1 & 4.870055000 & 6.259235000 & 0.925274000 \\
6 & 3.094750000 & 5.065218000 & 0.874601000 \\
6 & 2.930217000 & 5.003480000 & 2.365054000 \\
1 & 2.061704000 & 5.587432000 & 2.688727000 \\
1 & 3.813263000 & 5.408313000 & 2.863356000 \\
1 & 2.780861000 & 3.976196000 & 2.714666000 \\
7 & -0.255361000 & -0.280772000 & 1.088813000 \\
7 & -2.604497000 & -2.009269000 & -0.816905000 \\
7 & -3.911072000 & -2.076648000 & -0.906128000 \\
7 & -4.329409000 & -0.862882000 & -0.618809000 \\
7 & 2.128767000 & -4.226623000 & 1.143437000 \\
7 & 2.759809000 & -3.204131000 & 0.607671000 \\
7 & 0.069506000 & 4.313248000 & 1.093302000 \\
7 & 1.113646000 & 3.686249000 & 0.596527000 \\
7 & 1.010501000 & -3.717581000 & 1.607704000 \\
6 & 0.049446000 & -4.585629000 & 2.273998000 \\
1 & -0.084153000 & -4.247685000 & 3.302613000 \\
1 & -0.898152000 & -4.542867000 & 1.734505000 \\
1 & 0.449152000 & -5.597657000 & 2.255443000 \\
7 & -0.700609000 & 3.356259000 & 1.559321000 \\
6 & -1.950793000 & 3.707229000 & 2.219193000 \\
1 & -2.769551000 & 3.169725000 & 1.739631000 \\
1 & -1.887007000 & 3.434845000 & 3.273911000 \\
1 & -2.089993000 & 4.781540000 & 2.113244000 \\
6 & -1.824727000 & -3.198721000 & -1.140367000 \\
\hline & -2.527004000 & -4.017002000 & -1.286492000 \\
\hline & -1.261079000 & -3.018115000 & -2.056797000 \\
\hline
\end{tabular}

Trimethylated 3a (central amine + triazole).

\begin{tabular}{|c|c|c|c|}
\hline & & & \\
\hline & -8.016845000 & 000 & 2271000 \\
\hline & 000 & 0 & $50+2>$ \\
\hline & 000 & & \\
\hline & 000 & & 0 \\
\hline & 02000 & 4000 & 1900 \\
\hline & 8000 & 4000 & 300 \\
\hline & 1000 & & 000 \\
\hline & 000 & 00 & 000 \\
\hline & -8.958752000 & -2.698661000 & 273000 \\
\hline & -6.9 & -2 & $-U$. \\
\hline & -6.3 & 0 & 000 \\
\hline & -7.1 & 00 & 1000 \\
\hline & 000 & & 000 \\
\hline & 000 & -3 & -0 . \\
\hline & 0.2 & & \\
\hline & -0.56 & -0.7 & 000 \\
\hline & 0.8 & 00 & 00 \\
\hline & 1.1 & & \\
\hline & 2.5 & & \\
\hline & 3.2 & & 000 \\
\hline & 4.1 & & \\
\hline & 4.3 & & \\
\hline & 0 & & 000 \\
\hline & 00 & -5 . & -1 \\
\hline & 3.6 & & -3 \\
\hline & 2 & & \\
\hline & 0 & & \\
\hline & 5.83 & -5 & -2 \\
\hline & 6 & 0 & 00 \\
\hline & & & \\
\hline & & & \\
\hline & 7.8 & -6.5 & -1. \\
\hline & 0 & & \\
\hline & & & \\
\hline & 7.13 & & \\
\hline & 5.12 & -3.6 & 00 \\
\hline & & & \\
\hline & & & \\
\hline & 000 & -3.2 & 000 \\
\hline & 000 & -1.8 & 00 \\
\hline & 1.0 & & \\
\hline & -1.87 & & -0.0 \\
\hline & -1.408535000 & 52172 & -1.74 \\
\hline & -0.1 & & 00 \\
\hline & & & \\
\hline & & & -1.849057000 \\
\hline & 2.20 & 00 & 00 \\
\hline & 1.81 & & -0.1 \\
\hline & 0.37 & 0 & -0.0 \\
\hline & -0.02 & & \\
\hline & -0.25 & & - \\
\hline & 0.254783000 & & -0.38 \\
\hline & 2.8330 & 170000 & -0.2 \\
\hline & & & \\
\hline
\end{tabular}




$\begin{array}{lrrr}6 & 4.183253000 & 6.776270000 & -0.256896000 \\ 6 & 5.250875000 & 7.821685000 & -0.403203000 \\ 1 & 5.298174000 & 8.180874000 & -1.438184000 \\ 1 & 6.235529000 & 7.426719000 & -0.141172000 \\ 1 & 5.043063000 & 8.689600000 & 0.229920000 \\ 6 & 4.517029000 & 5.422665000 & -0.154253000 \\ 1 & 5.562621000 & 5.127354000 & -0.156591000 \\ 6 & 3.541209000 & 4.432486000 & -0.035605000 \\ 6 & 3.908922000 & 2.981524000 & 0.088300000 \\ 1 & 3.334034000 & 2.486821000 & 0.878290000 \\ 1 & 4.969952000 & 2.879441000 & 0.325663000 \\ 1 & 3.728105000 & 2.441857000 & -0.849112000 \\ 7 & -0.303888000 & -0.240583000 & -0.875586000 \\ 7 & -2.751610000 & -1.408468000 & 0.670424000 \\ 7 & -4.004024000 & -1.297662000 & 1.002863000 \\ 7 & -4.696549000 & -1.056750000 & -0.125726000 \\ 7 & 1.877932000 & -3.895579000 & 0.779201000 \\ 7 & 2.878178000 & -3.156792000 & 0.344117000 \\ 7 & -0.290079000 & 2.716673000 & 1.019504000 \\ 7 & 0.536896000 & 3.706651000 & 1.229834000 \\ 7 & 1.186498000 & 3.856654000 & 0.092519000 \\ 6 & 0.780706000 & -0.147733000 & -1.906526000 \\ 1 & 1.090340000 & -1.155106000 & -2.181486000 \\ 1 & 1.625469000 & 0.401896000 & -1.494592000 \\ 1 & 0.378741000 & 0.363356000 & -2.781447000 \\ 7 & 0.856317000 & -3.082740000 & 0.850074000 \\ 6 & -0.399173000 & -3.621690000 & 1.370438000 \\ 1 & -0.264884000 & -3.832825000 & 2.432394000 \\ 1 & -1.197022000 & -2.893671000 & 1.230071000 \\ 1 & -0.622209000 & -4.540288000 & 0.828185000 \\ 6 & -1.230508000 & 2.346327000 & 2.073185000 \\ 1 & -0.866230000 & 2.777591000 & 3.003483000 \\ 1 & -2.212446000 & 2.754275000 & 1.826843000 \\ 1 & -1.277872000 & 1.261124000 & 2.150274000\end{array}$

Open structure of $\left[\mathrm{Ag}_{3}(1 \mathrm{la})_{2}\right]^{3+}$.

$\begin{array}{lrrr}47 & -3.439063000 & -0.131932000 & 0.070547000 \\ 47 & 1.431619000 & 2.921344000 & 0.085698000 \\ 47 & 1.676937000 & -2.774147000 & -0.094281000 \\ 6 & -5.829827000 & -0.473416000 & -2.317483000 \\ 6 & -6.870907000 & 0.393486000 & -1.979207000 \\ 6 & -8.072863000 & -0.188910000 & -1.578107000 \\ 1 & -8.907186000 & 0.458673000 & -1.327072000 \\ 6 & -8.231401000 & -1.573600000 & -1.495455000 \\ 6 & -7.143756000 & -2.395273000 & -1.809948000 \\ 1 & -7.254026000 & -3.474649000 & -1.745784000 \\ 6 & -5.926556000 & -1.867107000 & -2.234307000 \\ 6 & -4.767761000 & -2.752497000 & -2.597713000 \\ 1 & -5.048728000 & -3.804729000 & -2.526899000 \\ 1 & -3.913416000 & -2.583219000 & -1.931056000 \\ 1 & -4.430880000 & -2.564453000 & -3.623145000 \\ 6 & -9.549071000 & -2.177405000 & -1.102127000 \\ 1 & -10.218023000 & -1.435564000 & -0.660237000 \\ 1 & -9.419250000 & -2.996691000 & -0.388539000\end{array}$

\begin{tabular}{|c|c|c|c|}
\hline & 1000 & 00 & \\
\hline & 96729000 & 1.883 & -2 \\
\hline & -5.855899000 & 2.204625000 & -1.41 \\
\hline & -7.594249000 & 2.389891000 & -1.678 \\
\hline & 6.502474000 & 000 & -3 \\
\hline & -3.479034000 & 3000 & 2000 \\
\hline & 61000 & 7000 & 000 \\
\hline & -2.625510000 & 1.311088000 & 0000 \\
\hline & 428000 & 4000 & 000 \\
\hline & -2.678408000 & 6000 & -5.3 \\
\hline & 6000 & $0.8 \mathrm{~s}$ & -6 . \\
\hline & -1.123554000 & 1.130632000 & -2.61 \\
\hline & 000 & 000 & -3 \\
\hline & 3000 & 000 & \\
\hline & -0.541230000 & 2000 & 3000 \\
\hline & -1.504657000 & -1.466049000 & 9000 \\
\hline & 000 & -1.2 & -1 \\
\hline & 0 & -2 & \\
\hline & 0.089829000 & -2.39 & -5.5 \\
\hline & 39000 & 000 & -5 \\
\hline & 000 & $-3 .($ & \\
\hline & 000 & -2 & \\
\hline & 1.370737000 & -2.84 & -2 \\
\hline & 000 & -4.4 & -2 \\
\hline & 3.0 & & \\
\hline & 00 & 00 & 000 \\
\hline & 000 & -7.6 & -1 \\
\hline & 000 & -6.0 & -1 \\
\hline & 5.5 & & \\
\hline & 000 & -4 & -1.88 \\
\hline & 7000 & -3.8259 & $-2.4 C$ \\
\hline & 0 & -2 & -2 \\
\hline & & & \\
\hline & 00 & -2 & 00 \\
\hline & 4.042368000 & -1.774429000 & -1.8 \\
\hline & 6.5 & -6.8 & -1 \\
\hline & 00 & & \\
\hline & 5000 & -7.85 & -1.1 \\
\hline & 7.175156000 & -6.4 & -0.7 \\
\hline & 00 & 00 & \\
\hline & & -5.9 & \\
\hline & 1.678147000 & -7.494287000 & -2.305769000 \\
\hline & .293428000 & -6.347924000 & -3.5 \\
\hline & 0 & & \\
\hline & 00 & 0.27 & -1.6 \\
\hline & 5000 & -0.18900 & -3.2 \\
\hline & 3000 & 1.898169000 & -2 \\
\hline & & 1.807 & 68000 \\
\hline & 1.680157000 & 2.553128000 & -6.201373000 \\
\hline & 1.074518000 & 1.037546000 & -5.457992000 \\
\hline & 2.831467000 & 1.354 & $-5.55604 \mathrm{~S}$ \\
\hline & 2000 & 2.923 & -1.97 \\
\hline & 1.936072000 & 5.382094000 & -2.312626000 \\
\hline & 0.733889000 & 6.099484000 & -2.272678000 \\
\hline & 0.793575000 & 7.424052000 & -1.846247000 \\
\hline
\end{tabular}




\begin{tabular}{|c|c|c|c|}
\hline & 0 & & \\
\hline & 0 & 0 & \\
\hline & 172347000 & 5000 & - \\
\hline & 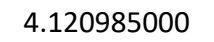 & 0 & - \\
\hline & & & \\
\hline & 0 & & \\
\hline & 0 & & \\
\hline & & & \\
\hline & & & \\
\hline & 2.04 & 000 & 000 \\
\hline & 0 & 000 & 000 \\
\hline & 00 & & \\
\hline & 0 & 00 & 00 \\
\hline & -0.5 & 00 & -2 \\
\hline & -0. & & \\
\hline & -1.3 & & 000 \\
\hline & -0.7 & 00 & 000 \\
\hline & 000 & & 000 \\
\hline & -6.7 & 00 & 000 \\
\hline & -7.9 & & \\
\hline & -8.7 & 00 & 000 \\
\hline & -8.2 & & \\
\hline & -7.2 & & \\
\hline & & & \\
\hline & -5.9 & & \\
\hline & -4.9 & & \\
\hline & -4 . & & \\
\hline & 0 & & \\
\hline & -4. & & \\
\hline & -9.6 & 00 & \\
\hline & & & \\
\hline & 000 & & \\
\hline & -10 & & 0 \\
\hline & 0 & & \\
\hline & & & \\
\hline & & & \\
\hline & -6.15 & $-2.7 €$ & 000 \\
\hline & -3. & 0 & \\
\hline & & & \\
\hline & -2.37 & & \\
\hline & -1.3 & & \\
\hline & 0 & & \\
\hline & & & \\
\hline & -0.953220000 & -1.213780000 & 2.697079000 \\
\hline & -0.63 & -2.17 & 00 \\
\hline & & & \\
\hline & & & \\
\hline & -0.72 & 00 & \\
\hline & 000 & 1.361780000 & 000 \\
\hline & & .2457 & \\
\hline & 0000 & 917640000 & \\
\hline & 0 & 0 & \\
\hline & ( & 1.252096000 & \\
\hline & -0.7 & 2.890785000 & 5.731587 \\
\hline & & & \\
\hline
\end{tabular}

\begin{tabular}{|c|c|c|c|}
\hline & & 0 & \\
\hline & 672000 & 09000 & \\
\hline & .483747000 & 677631000 & 7000 \\
\hline & .486516000 & 271310000 & 2.346047000 \\
\hline & 0 & 000 & 5000 \\
\hline & 0 & 00 & 000 \\
\hline & 00 & 000 & 2000 \\
\hline & 0 & 000 & 000 \\
\hline & & 000 & \\
\hline & 0 & 000 & 000 \\
\hline & .000 & 7.386006000 & 1000 \\
\hline & 021000 & 000 & 1000 \\
\hline & 00 & 000 & \\
\hline & 0 & 00 & 000 \\
\hline & 6000 & 000 & 000 \\
\hline & 7.2 & 000 & 000 \\
\hline & 000 & 000 & 000 \\
\hline & 000 & 000 & 000 \\
\hline & 3.966486000 & 5000 & 8000 \\
\hline & 0 & & \\
\hline & & & \\
\hline & 0 & 00 & 000 \\
\hline & 000 & 000 & 000 \\
\hline & 0 & 00 & 000 \\
\hline & 0 & 00 & \\
\hline & 8000 & 665000 & 000 \\
\hline & 00 & -1.0 & 000 \\
\hline & & & \\
\hline & 000 & -2.7 & \\
\hline & 000 & 000 & 000 \\
\hline & 000 & 1480000 & 2000 \\
\hline & 0 & & 000 \\
\hline & 0 & -7.9 & 000 \\
\hline & 000 & -7.6 & 000 \\
\hline & & & \\
\hline & & & \\
\hline & 1000 & -5.46 & 1000 \\
\hline & 5.048874000 & -4.461721000 & 1.919741000 \\
\hline & 000 & -4.8 & 000 \\
\hline & & & \\
\hline & .838273000 & -3.5695 & 9000 \\
\hline & 00 & $-9 . c$ & 000 \\
\hline & 3.288542000 & -9.783639000 & 24000 \\
\hline & 4.387456000 & -9.140813000 & 0.21 \\
\hline & 2.661616000 & -9.334758000 & -0.147630000 \\
\hline & 0.155706000 & -5.603391000 & 98000 \\
\hline & 0.109968000 & -5.317422000 & 71000 \\
\hline & -0.548302000 & -6.422943000 & 2.255520000 \\
\hline & -0.184130000 & -4.745487000 & 1.817268000 \\
\hline & 2.127585000 & -2.347499000 & 4.120596000 \\
\hline & 2.483388000 & -3.604617000 & 3.989810000 \\
\hline & 2.363463000 & -3.827680000 & 2.690386000 \\
\hline & -4.576970000 & 0.086903000 & 2.17700200 \\
\hline & -4.406734000 & 0.404216000 & -4.020390000 \\
\hline & & & \\
\hline
\end{tabular}




$\begin{array}{lrrr}7 & -0.170076000 & 0.150029000 & -3.164848000 \\ 7 & 0.773409000 & -2.694705000 & -4.261513000 \\ 7 & 1.765295000 & -3.554361000 & -4.261055000 \\ 7 & 2.107996000 & -3.633600000 & -2.984885000 \\ 7 & 1.771349000 & 2.466832000 & -4.131198000 \\ 7 & 1.971502000 & 3.761776000 & -4.054442000 \\ 7 & 1.877025000 & 4.015751000 & -2.757525000 \\ 7 & -4.476647000 & -0.453647000 & 2.911162000 \\ 7 & -4.253532000 & -0.764130000 & 4.177470000 \\ 7 & -2.979137000 & -1.077634000 & 4.199749000 \\ 7 & 0.774623000 & 2.684374000 & 4.296022000 \\ 7 & 1.740463000 & 3.572390000 & 4.263552000 \\ 7 & 2.003945000 & 3.696567000 & 2.972198000 \\ 7 & -0.072630000 & -0.164007000 & 3.241118000 \\ 7 & 3.225463000 & 0.181512000 & 0.021175000 \\ 6 & 4.166303000 & 0.858207000 & -0.056123000 \\ 6 & 5.331861000 & 1.718935000 & -0.151290000 \\ 1 & 5.166224000 & 2.632386000 & 0.428535000 \\ 1 & 5.513480000 & 1.989296000 & -1.194432000 \\ 1 & 6.213955000 & 1.204640000 & 0.239091000\end{array}$

Closed structure of $\left[\mathrm{Ag}_{3}(1 \mathrm{a})_{2}\right]^{3+}$.

$\begin{array}{llll}47 & 1.762462000 & -0.601514000 & 0.275458000 \\ 47 & -0.040047000 & 1.929726000 & 0.027239000 \\ 47 & -1.755440000 & -0.707674000 & -0.272638000 \\ 6 & 1.466724000 & 1.670281000 & -2.828675000 \\ 1 & 1.124564000 & 2.598273000 & -2.354808000 \\ 1 & 1.665969000 & 1.913372000 & -3.889189000 \\ 6 & 2.738694000 & 1.213304000 & -2.190012000 \\ 6 & 2.971551000 & 0.336629000 & -1.137323000 \\ 6 & 4.269123000 & 2.384323000 & -3.853982000 \\ 1 & 5.335058000 & 2.599260000 & -3.832951000 \\ 1 & 3.708506000 & 3.316015000 & -3.808987000 \\ 1 & 4.022495000 & 1.825794000 & -4.760300000 \\ 6 & 5.223192000 & -0.617229000 & -0.384298000 \\ 6 & 6.065901000 & -1.435565000 & -1.156964000 \\ 6 & 7.068146000 & -2.147314000 & -0.501234000 \\ 1 & 7.739164000 & -2.764415000 & -1.091912000 \\ 6 & 7.236277000 & -2.084970000 & 0.881524000 \\ 6 & 6.314071000 & -1.341684000 & 1.617646000 \\ 1 & 6.395277000 & -1.319994000 & 2.701580000 \\ 6 & 5.295795000 & -0.601701000 & 1.017811000 \\ 6 & 8.369662000 & -2.793102000 & 1.563676000 \\ 6 & -0.825667000 & 1.165501000 & -3.352847000 \\ 1 & -1.473192000 & 0.292613000 & -3.502138000 \\ 1 & -0.609559000 & 1.570276000 & -4.359051000 \\ 6 & -1.620510000 & 2.161982000 & -2.563060000 \\ 6 & -1.705608000 & 2.401921000 & -1.196839000 \\ 6 & -2.950919000 & 2.986952000 & -4.577504000 \\ 1 & -3.774475000 & 3.692723000 & -4.670569000 \\ 1 & -3.258123000 & 2.007081000 & -4.948833000 \\ 1 & -2.091438000 & 3.348092000 & -5.145107000 \\ 6 & -3.523646000 & 3.769707000 & 0.025681000 \\ 6 & -4.909720000 & 3.543274000 & 0.134148000\end{array}$

\begin{tabular}{|c|c|c|c|}
\hline & & & \\
\hline & 000 & 000 & 000 \\
\hline & .935015000 & 0 & 192472000 \\
\hline & 0 & & \\
\hline & -3.0 & & 000 \\
\hline & 3000 & 00 & 000 \\
\hline & 389000 & & 000 \\
\hline & 00 & & 000 \\
\hline & 00 & -0.8 & 000 \\
\hline & 0.843644000 & -0.549563000 & -4.3 \\
\hline & 939000 & -1.7 & 000 \\
\hline & -1.0 & -1.8 & \\
\hline & 0.700168000 & -3.2 & 00 \\
\hline & 000 & -4.3 & 000 \\
\hline & 00 & -3 . & 00 \\
\hline & 00 & -2 & \\
\hline & -2.652292000 & -3.7 & -1.4 \\
\hline & -3.820378000 & -3.8 & -2.1 \\
\hline & -4.5 & -4.4 & \\
\hline & -5.8 & -4 . & \\
\hline & 772000 & -4.9 & 000 \\
\hline & 1000 & -4 & 000 \\
\hline & -3.6 & -5.1 & \\
\hline & -2.5 & -4.2 & -0 \\
\hline & -6.10 & -5.6 & 000 \\
\hline & -0.8 & -0.6 & \\
\hline & & & \\
\hline & -1.8 & & \\
\hline & 0.12 & -1.7 & 00 \\
\hline & 1.09 & -1.8 & 1.8 \\
\hline & & & \\
\hline & -0.40 & & \\
\hline & -1.73 & -3.4 & 00 \\
\hline & -0.5 & -2 & \\
\hline & & & \\
\hline & 3.888813000 & -3.85 & 2.2 \\
\hline & 5.033647000 & -4.4 & 00 \\
\hline & & & \\
\hline & & & \\
\hline & 4.075833000 & -4.504 & 000 \\
\hline & 000 & -4.7 & 00 \\
\hline & 2.892071000 & & \\
\hline & & & \\
\hline & 1.409216000 & 0.257 & 25077 \\
\hline & 0 & 0 & 00 \\
\hline & & & \\
\hline & 1.621108000 & 2.398599000 & 1.261177000 \\
\hline & 2.860723000 & 2.926 & 00 \\
\hline & 3.679393000 & 3.63611 & 4.760863000 \\
\hline & 332000 & 1.94 & 000 \\
\hline & 1.998351000 & 3.2707 & 0 \\
\hline & $3.420158 c$ & 3.81 & 00 \\
\hline & 2.735728000 & 4.646833000 & -0.839515000 \\
\hline & 3.440998000 & 5.208563000 & -1.900612000 \\
\hline & & & \\
\hline
\end{tabular}




\begin{tabular}{|c|c|c|c|}
\hline & & & \\
\hline & 000 & 000 & \\
\hline & 000 & 0 & \\
\hline & 0 & & \\
\hline & 0 & 00 & -3.1 \\
\hline & 000 & 000 & 2.85 \\
\hline & 3000000 & 43000 & \\
\hline & 000 & 000 & \\
\hline & 00 & 00 & 2.1 \\
\hline & -3.002981000 & 69000 & 1.1 \\
\hline & -4.38 & & \\
\hline & 0 & & \\
\hline & -3.8 & 000 & \\
\hline & 000 & 000 & \\
\hline & -5.2 & 000 & \\
\hline & -6.06 & 00 & \\
\hline & -7.045579000 & 08000 & 0.2 \\
\hline & -7.70879 & -2.9 & $0 . c^{2}-1$ \\
\hline & -7.2 & -2 & \\
\hline & -6.2 & 0 & \\
\hline & 2000 & -1.2 & \\
\hline & 00 & -0.6 & \\
\hline & -8.3 & & \\
\hline & 0.35 & 00 & -2.7 \\
\hline & 3.95 & 00 & -2 \\
\hline & 0 & & -2 \\
\hline & & & \\
\hline & 0 & & \\
\hline & & & -2 \\
\hline & -2.79 & 3.2 & -1.1 \\
\hline & & & \\
\hline & -0.9 & & \\
\hline & -1.5 & -3.1 & \\
\hline & -0.4 & & \\
\hline & & & \\
\hline & & & \\
\hline & 1.615 & -3.0 & 1.9 \\
\hline & & & \\
\hline & & & \\
\hline & 2.703009000 & 269560000 & 1.204 \\
\hline & -4.02 & 00 & \\
\hline & & & \\
\hline & -4.37 & & \\
\hline & -5.96130 & 5.691530000 & 0284411 \\
\hline & -7.0 & 00 & $-c$ \\
\hline & -6.2 & & \\
\hline & -8.601850000 & -3.781674000 & -1.331926 \\
\hline & -8.042151000 & -3.071475000 & 2.8 \\
\hline & -9.2 & 00 & 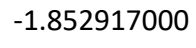 \\
\hline & 6.36 & -5.50 & -0.0 \\
\hline & & -5.2 & -1.11 \\
\hline & 6.202417000 & 0 & \\
\hline & 7.236925000 & 09000 & \\
\hline & 8.86650 & 2000 & $0.8 s$ \\
\hline & & & \\
\hline
\end{tabular}

\begin{tabular}{|c|c|c|c|}
\hline & & & \\
\hline & 000 & 3000 & \\
\hline & 7000 & 000 & \\
\hline & 967460000 & .760253000 & -4.089 \\
\hline & 00 & 00 & . \\
\hline & & & \\
\hline & 000 & 000 & 3.0 \\
\hline & 00 & 000 & 2.4 \\
\hline & 00 & & \\
\hline & 000 & .000 & 2.7 \\
\hline & 80000 & -1.089525000 & 3.0 \\
\hline & -4.321751000 & 2000 & -1.9 \\
\hline & -3.84 & & -1.3 \\
\hline & 00 & 00 & -2.3 \\
\hline & 07000 & 0.599697000 & $-2 . \varepsilon$ \\
\hline & 000 & 00 & \\
\hline & -5.3 & & \\
\hline & -6.0 & 00 & -0. \\
\hline & -6.702140000 & 00 & -0.9 \\
\hline & -1.4 & 00 & \\
\hline & & & \\
\hline & -1.2 & & -0 \\
\hline & -1.1 & & \\
\hline & 0 & & -0 . \\
\hline & 00 & 00 & \\
\hline & 1.121 & 00 & 0.3 \\
\hline & 1.0 & & -1.2 \\
\hline & & & \\
\hline & & & \\
\hline & 6.5 & & \\
\hline & 00 & & \\
\hline & & & \\
\hline & 0 & & \\
\hline & 0 & 00 & \\
\hline & & & \\
\hline & & & \\
\hline & 6.449762000 & -0.80606 & \\
\hline & 6.309563000 & -2.550432000 & -2.9 \\
\hline & 0 & 00 & 2. \\
\hline & & & \\
\hline & 0.950955000 & -3.133676000 & -0.5 \\
\hline & 2.16 & & \\
\hline & 1.395356000 & -4.646313000 & -1.3 \\
\hline & 3.840069000 & & \\
\hline & .312317000 & -4.191053000 & 4346 \\
\hline & 852334000 & -3.3 & 4.1 \\
\hline & & & \\
\hline & -1.305467000 & -4.196872000 & 0.6732660 \\
\hline & -1.482192000 & -4.571511000 & 1.683 \\
\hline & -0.538839000 & -4.831944000 & 0.22104 \\
\hline & -0.899731000 & -3.182701000 & 0.7411390 \\
\hline & -3.886173000 & -3.288982000 & -3.60180 \\
\hline & -3.374646000 & -2.325111000 & -3.697447 \\
\hline & -3.418429000 & -3.981139000 & -4.309022 \\
\hline & & & \\
\hline
\end{tabular}


Open structure of $\left[\mathrm{Ag}_{3}(1 \mathrm{~b})_{2}\right]^{3+}$.

\begin{tabular}{|c|c|c|c|}
\hline & & & \\
\hline & 0.000127000 & 3.362679000 & -0.0000 \\
\hline & 3.049197000 & -1.722358000 & -0.022100000 \\
\hline & -5.237996000 & -2.459138000 & -2.693293000 \\
\hline & -6.088989000 & -2.268981000 & 065000 \\
\hline & -7.147875000 & -3.150809000 & 89000 \\
\hline & -7.837492000 & -3.014209000 & -0.601696000 \\
\hline & -7.332488000 & -4.207470000 & -2.319725000 \\
\hline & -6.477788000 & -4.378259000 & -3.4 \\
\hline & -6.641572000 & 000 & -4. \\
\hline & -5.421130000 & -3.4960 & 6000 \\
\hline & -8.438993000 & 573000 & -2.06 \\
\hline & -3.237719000 & 37000 & 86000 \\
\hline & -2.438611000 & -0.2694 & 1000 \\
\hline & -2.489398000 & 0.496380000 & -5.142237000 \\
\hline & -1.402085000 & 0.560252000 & -5.105300000 \\
\hline & -2.92 & & -5.1 \\
\hline & -2.817 & 000 & $-6 . c$ \\
\hline & -1.251106000 & 0.531358000 & 8000 \\
\hline & -1.37023 & 000 & 000 \\
\hline & -1.22 & 0.5 & -1.2 \\
\hline & 0.22987 & 000 & -2 \\
\hline & -0.615613000 & -1.965822000 & -2.64 \\
\hline & 0.23 & -1.3 & -1.2 \\
\hline & 1.514314000 & & \\
\hline & 0.878155000 & -2.385171000 & -5.185601000 \\
\hline & 0.277 & -1.4 & -5.1 \\
\hline & 0.233785000 & $-3.26 t$ & -5.1 \\
\hline & $1.4 \varepsilon$ & -2.4 & -6.0 \\
\hline & 2.653299000 & -2.211929000 & -2.011 \\
\hline & 4.823198000 & -3.251729000 & -2.737 \\
\hline & 5.091603000 & -4.073554000 & -1.645083000 \\
\hline & 6.390012000 & & \\
\hline & 6.622531000 & -5.189393000 & -0.6 \\
\hline & 7.395100000 & -4.155867000 & -2.350994000 \\
\hline & 7.10774 & -3.3 & \\
\hline & 7.895874000 & -3.069955000 & -4.137563000 \\
\hline & 5.809893000 & -2.886379000 & -3.647171000 \\
\hline & 8.815646000 & -4.592932000 & -2.087420000 \\
\hline & 1.13 & 0000 & -2 \\
\hline & 000 & 0.844480000 & -1.3 \\
\hline & 2.048278000 & 0.480735000 & -2.931284000 \\
\hline & 0.949709000 & 2.312803000 & -2.827934000 \\
\hline & 1.727763000 & 2.229974000 & -5.247826000 \\
\hline & 1.474098000 & 2.846897000 & -6.108539000 \\
\hline & 1.280724000 & 1.240608000 & -5.344636000 \\
\hline & 2.813633000 & 2.145188000 & -5.164161000 \\
\hline & 0.505156000 & 3.336594000 & -2.006528000 \\
\hline & 0.106224000 & 5.746976000 & -2.512883000 \\
\hline & -1.059687000 & 5.902725000 & -1.762403000 \\
\hline & -1.463017000 & 7.180801000 & -1.405911000 \\
\hline & -2.372086000 & 7.323999000 & -0.832265000 \\
\hline & & 307000 & -1.811480 \\
\hline
\end{tabular}

\begin{tabular}{|c|c|c|c|}
\hline & & & \\
\hline & 000 & 000 & 00 \\
\hline & 868692000 & .836651000 & 2. \\
\hline & 0 & 00 & -1 \\
\hline & 3000 & 000 & 00 \\
\hline & -5.092015000 & 6000 & 1. \\
\hline & 470000 & 000 & \\
\hline & 000 & 000 & \\
\hline & 9000 & 000 & 00 \\
\hline & -7.108047000 & -3.341664000 & 3.4 \\
\hline & -7.89 & -3 & \\
\hline & -5.8 & & \\
\hline & -8.81 & 00 & 00 \\
\hline & 000 & 000 & \\
\hline & -1.5 & 00 & \\
\hline & -0.8 & & \\
\hline & -0.277307000 & -1.476 & 5 \\
\hline & -0.234149000 & -3.2 & \\
\hline & -1.4 & & \\
\hline & -0.2 & & \\
\hline & 00 & -1. & \\
\hline & -0.23 & -1 & \\
\hline & -1.1 & & \\
\hline & -1.2 & 00 & \\
\hline & -2.04 & 00 & \\
\hline & -0.9 & & \\
\hline & -0.5 & & \\
\hline & -1.72 & & \\
\hline & -1.280 & 1.2 & \\
\hline & -2.8 & 0 & \\
\hline & & & \\
\hline & -0.1 & & \\
\hline & 1.06 & & \\
\hline & 1.4 & & \\
\hline & 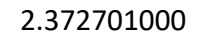 & & \\
\hline & 0.7132 & 8.28 & \\
\hline & -0.4471 & 8.11 & \\
\hline & & & \\
\hline & -0.86844 & & \\
\hline & 1.232242000 & 33000 & \\
\hline & 1.25 & & \\
\hline & $U$ & & \\
\hline & 942000 & & \\
\hline & 2.438605000 & 2697 & 00 \\
\hline & 2.48 & & \\
\hline & & & \\
\hline & 1.402063000 & 0.560046000 & 5.105224000 \\
\hline & 2.928 & 00 & 5.1 \\
\hline & 3.237636000 & 328000 & \\
\hline & 5.237 & -2.45 & \\
\hline & 5.420871000 & -3.49664 & \\
\hline & 6.47 & & \\
\hline & 6.641186000 & -5.196296000 & \\
\hline & 7.332232000 & -4.208035000 & 2.31 \\
\hline & & 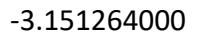 & \\
\hline
\end{tabular}




\begin{tabular}{|c|c|c|c|}
\hline & 000 & -3.014 & 0.6 \\
\hline & 6.088917000 & -2.269367000 & 1.607134000 \\
\hline & 8.438656000 & -5.203210000 & 2.066287000 \\
\hline & 2.934741000 & -0.280195000 & 94247000 \\
\hline & 3.985939000 & -1.052356000 & 4.113007000 \\
\hline & 4.155537000 & -1.545682000 & 2.896903000 \\
\hline & -4.155633000 & -1.545281000 & -2.89 \\
\hline & -3.986049000 & 5000 & -4.11 \\
\hline & -2.934792000 & -0.279851000 & 19000 \\
\hline & 0.010752000 & 0.014436000 & -2.869827000 \\
\hline & 1.773309000 & -2.379355000 & -4.037873000 \\
\hline & 2.973230000 & -2.891573000 & -4.157923000 \\
\hline & 3.486038000 & -2.785553000 & -2.942244000 \\
\hline & 1.189040000 & 2.867256000 & -4.054642000 \\
\hline & 0.926611000 & 4.15 & 0000 \\
\hline & 0.515815000 & 4.417558000 & 37000 \\
\hline & -3.486323000 & -2.785250000 & 2.942197000 \\
\hline & -2.973571000 & -2.891262000 & 4.157901000 \\
\hline & -1.773591000 & -2.379180000 & 4.037875000 \\
\hline & -1.188777000 & 2.86 & 000 \\
\hline & -0.926350000 & 4.153307000 & 4.081239000 \\
\hline & -0.515592000 & 4.417565000 & 2.850905000 \\
\hline & -0.010726000 & 0.0 & 000 \\
\hline & 1.503079000 & 9.737292000 & 0.145 \\
\hline & 0.362022000 & 10.621605000 & 1.776016000 \\
\hline & 2.378935000 & 9.897820000 & 2.118836000 \\
\hline & 35000 & 9.898 & -2.1 \\
\hline & -1.501954000 & 9.7373 & -0.14 \\
\hline & -0.361010000 & 10.621598000 & -1.775828000 \\
\hline & 8.866741000 & -5.858971000 & -1.664933000 \\
\hline & 9.581806000 & -4.471279000 & 03000 \\
\hline & 9.355181000 & -3.816222000 & -1.119036000 \\
\hline & 8.056315000 & -6.069063000 & 1.098660000 \\
\hline & 9.553646000 & -4.597765000 & 1.648742000 \\
\hline & 8.721071000 & -5.918878000 & 3.158903000 \\
\hline & -9.355752000 & -3.815470000 & 1.119531000 \\
\hline & -8.867275000 & -5.858314000 & 1.665038000 \\
\hline & -9.582068000 & -4.470836000 & 3.176027000 \\
\hline & -9.553968000 & -4.597049000 & 996000 \\
\hline & -8.721398000 & -5.918218000 & -3.159099000 \\
\hline & -8.056777000 & -6.068443000 & -1.098812000 \\
\hline & -5.565856000 & -2.258525000 & 4.497004000 \\
\hline & -4.293883000 & -4.373339000 & 0.974408000 \\
\hline & -5.944644000 & -1.428095000 & -0.936929000 \\
\hline & -4.751070000 & -3.608022000 & -4.447368000 \\
\hline & 4.293427000 & -4.373602000 & -0.974416000 \\
\hline & 5.565664000 & -2.259112000 & -4.497109000 \\
\hline & 4.750780000 & -3.608611000 & 4.447240000 \\
\hline & 5.944643000 & -1.428409000 & 0.937075000 \\
\hline & 1.772395000 & 6.689040000 & -3.498616000 \\
\hline & -1.657843000 & 5.038895000 & -1.492177000 \\
\hline & 1.658142000 & 5.038731000 & 1.492217000 \\
\hline & -1.772272000 & 6.689144000 & 3.498127000 \\
\hline
\end{tabular}

Closed structure of $\left[\mathrm{Ag}_{3}(\mathbf{1 b})_{2}\right]^{3+}$.

\begin{tabular}{|c|c|c|c|}
\hline & & & \\
\hline & 0.845740000 & -1.646744000 & 0 \\
\hline & 1.514908000 & 1.060210000 & -0.504006000 \\
\hline & -0.323117000 & -2.316035000 & -2.799532000 \\
\hline & 0.314659000 & -2.960847000 & 6000 \\
\hline & -0.408815000 & -2.803422000 & 96000 \\
\hline & -1.673111000 & -2.212321000 & -2.166410000 \\
\hline & -2.144714000 & -1.352783000 & -1.180810000 \\
\hline & -2.759195000 & -4.0 & 00 \\
\hline & -3.699 & -4.5 & 000 \\
\hline & -1.931957000 & 7000 & 000 \\
\hline & -2.703647000 & -3.604077000 & 000 \\
\hline & -4.451369000 & 64000 & 4000 \\
\hline & -5.780597000 & -1.191933000 & 000 \\
\hline & -6.058953000 & -1.517579000 & -1.584893000 \\
\hline & -6.740526000 & 82000 & 0.2 \\
\hline & -7.77 & -0.6 & \\
\hline & 000 & 000 & 00 \\
\hline & -5.031446000 & 3000 & 000 \\
\hline & 000 & 4000 & 00 \\
\hline & -4.0 & -0.8 & \\
\hline & -3.032775000 & 00 & 000 \\
\hline & -7.373669000 & 1000 & 000 \\
\hline & -7.1 & 0.0 & 00 \\
\hline & -8.62 & & \\
\hline & -7.301523000 & 1.725625000 & 7000 \\
\hline & 1.700953000 & -1.097190000 & -3.32 \\
\hline & 2.0334 & -0.07 & -3.5 \\
\hline & 1.80 & 0 & 00 \\
\hline & 2.618125000 & -1.637358000 & 3000 \\
\hline & 2.4410 & -1.8 & 000 \\
\hline & 4.605644000 & -1.8 & 306000 \\
\hline & 5.6723 & & \\
\hline & 4.423193000 & -0.897648000 & -4.32 \\
\hline & 4.242432000 & -2.672770000 & -4.482482000 \\
\hline & 4.15 & & \\
\hline & 5.442927000 & -2.178336000 & 61000 \\
\hline & 6.107136000 & -1.715537000 & 0.455188000 \\
\hline & 5.857299000 & -2.4 & 2000 \\
\hline & 6.85 & -2.1 & \\
\hline & 4.986865000 & -3.01821 & 3.3 \\
\hline & 3.713619000 & -3.423585000 & 2.982621000 \\
\hline & 3.061771000 & -3.942647000 & 3.6774 \\
\hline & 3.300756000 & -3.197554000 & \\
\hline & 2.332145000 & -3.542593000 & 1.340517000 \\
\hline & 5.374970000 & -3.202337000 & 4.833078000 \\
\hline & 4.670639000 & -2.320873000 & 5.597662000 \\
\hline & 6.671188000 & -2.965906000 & 5.040894000 \\
\hline & 5.075311000 & -4.425175000 & 5.272185000 \\
\hline & -0.426580000 & -0.070882000 & -3.706135000 \\
\hline & -1.491990000 & -0.310858000 & -3.613273000 \\
\hline & -0.179349000 & -0.159770000 & -4.779279000 \\
\hline & & & \\
\hline
\end{tabular}




\begin{tabular}{|c|c|c|c|}
\hline & 00 & 0 & -2 \\
\hline & -1.539545000 & 2.389599000 & -5.155803000 \\
\hline & 1.927222000 & 3.393720000 & 2000 \\
\hline & 0 & 0 & \\
\hline & 0000 & 00 & 000 \\
\hline & 000 & 4.15 & 000 \\
\hline & 1.630427000 & 276509000 & 8000 \\
\hline & 1000 & 3.6 & \\
\hline & 1.882 & 00 & 00 \\
\hline & 00 & 5.3 & 000 \\
\hline & 0.864202000 & 6.0 & 000 \\
\hline & 480000 & & \\
\hline & -1.1 & 6.7 & \\
\hline & -0.664773000 & 5.063924000 & 8000 \\
\hline & -1.646258000 & 4.99 & -1 \\
\hline & 00 & & \\
\hline & 00 & 7.6 & 00 \\
\hline & 2.019853000 & 000 & 1.3 \\
\hline & 1.757118000 & 6.467833000 & 2.85 \\
\hline & 0.6325 & & \\
\hline & 0.732150000 & 2.745 & 3.6 \\
\hline & 1.484215000 & 2.60 & 2.06 \\
\hline 6 & -0.63 & & \\
\hline & -1.3 & & \\
\hline & -1.02 & 4.7 & 000 \\
\hline & -1.656168000 & 5.6 & 000 \\
\hline+ & 024147000 & 5.06 & 00 \\
\hline & -1.2 & & \\
\hline 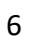 & -3.55 & 2.7 & 00 \\
\hline 6 & -4.85 & 2.8 & 000 \\
\hline \pm & -5 & 0 & \\
\hline & -5.91 & & \\
\hline & -6.925897000 & 2.548855000 & 000 \\
\hline 6 & -5.659987000 & 2.11590 & -1.6 \\
\hline v & 00 & .07 & -2 \\
\hline 1 & -4.17 & bo & \\
\hline 6 & -3.295453000 & 2.407286000 & -1.3 \\
\hline 1 & -2.27 & 0 & -1 \\
\hline & 392000 & & \\
\hline 6 & -0.37 & 0.2717 & 00 \\
\hline 1 & -1.227923000 & .95865 & 00 \\
\hline 1 & -0.085 & po & 00 \\
\hline 6 & -0.858739000 & & \\
\hline 6 & -0.564114000 & -1.820445000 & 1.986788000 \\
\hline 6 & -2.518180000 & -1.342446000 & 61000 \\
\hline 1 & -3.323022000 & -2.053 & 5.2 \\
\hline 1 & -2.924579000 & -0.33 & 4.94 \\
\hline 1 & -1.797916000 & -1.3 & 5.84 \\
\hline 6 & -1.684751000 & -3.9200 & 1.173850000 \\
\hline 6 & -0.597328000 & -4.552074000 & 5000 \\
\hline 1 & 0.412280000 & -4.281042000 & 0.856492000 \\
\hline 6 & -0.826102000 & -5.549879000 & -0.362379000 \\
\hline 1 & 0.01000 & -6.057819000 & -0.830900 \\
\hline b & 135046000 & -5.919155000 & -0.678872 \\
\hline
\end{tabular}

\begin{tabular}{|c|c|c|c|}
\hline & 0 & 0 & \\
\hline & $4.2 \angle 803$ & 5.597700000 & \\
\hline & 2.993008000 & -4.291594000 & 088043 \\
\hline & 2928000 & 00 & 1.36760200 \\
\hline & -2.365450000 & 3000 & -1.6 \\
\hline & $-1.45 \mathrm{~s}$ & 2000 & -2 \\
\hline & -3.587778000 & -6.940665000 & -2.2 \\
\hline & -2.2 & & \\
\hline & 1.98 & 0.3 & \\
\hline & 1.90 & 000 & 3.2 \\
\hline & 2.237574000 & 0.705216000 & \\
\hline & 000 & oo & \\
\hline & 3.10 & 0 & 08 \\
\hline & 4.921300000 & 0.608 & 4.03 \\
\hline & 6.00 & & \\
\hline & 4.6 & & \\
\hline & 4.5 & 00 & 4.6 \\
\hline & 5.119596000 & 000 & -0.65 \\
\hline & 2000 & 00 & -0.7 \\
\hline & 7.02 & & \\
\hline & 7.124296000 & 0.774 & -1.9 \\
\hline & 8.17850 & 0.529 & -2.0 \\
\hline & 6.42 & & \\
\hline & 5.0 & & \\
\hline & 4.54 & 00 & -3.8 \\
\hline & 000 & 00 & -1.7 \\
\hline & 000 & 00 & -1.7 \\
\hline & 7.09 & 0 & -4 \\
\hline & 6.81 & -0. & -5. \\
\hline & 8.42 & 00 & -4.3 \\
\hline & 6.6 & & \\
\hline & -6.4 & & \\
\hline & -7.027922000 & 00 & -3.4 \\
\hline & -7.916637000 & 1.472147000 & -1.87 \\
\hline & 318 & 00 & $-2 . \varepsilon$ \\
\hline & -2.719 & -3.0 & -2 \\
\hline & -3.812278000 & -2.74 & -1.87 \\
\hline & -3.45 & -1.7 & \\
\hline & 3.928831 & & \\
\hline & 4.606289000 & -2.274275 & -1.50 \\
\hline & 3.708610000 & -2.247124000 & -0.530336000 \\
\hline & -0.810893000 & 2.390886000 & -3.8 \\
\hline & -0.63 & 0 & -3 \\
\hline & 0.064 & 3.18 & -2.1 \\
\hline & 0.688 & 0.81 & 2.7 \\
\hline & -1.332922000 & 3.819774000 & \\
\hline & -2.438390000 & 3.986325000 & 1.72233700 \\
\hline & -2.445510000 & 2.952888000 & 0.89547300 \\
\hline & -1.866327000 & -1.712904000 & 3.779 \\
\hline & -2.243950000 & -2.811356000 & 3.17 \\
\hline & -1.459305000 & -2.863137000 & 2.10792600 \\
\hline & 4.392616000 & 0.691517000 & 2.6733980 \\
\hline & 5.229996000 & 0.868329000 & 1.68672400 \\
\hline & 4.453693000 & .932702000 & 0.612307 \\
\hline
\end{tabular}




\section{$\underline{\text { References }}$}

[1] S. W. Kwok, J. R. Fotsing, R. J. Fraser, V. O. Rodionov and V. V. Fokin, Org. Lett., 2010, $12,4217-4219$

[2] F. Sebest, L. Casarrubios, H. S. Rzepa, A. J. P. White and S. Díez-González, Green Chem., 2018, 20, 4023-4035.

[3] LIFE and BRAIN GMBH. 8-Triazolylxanthine Derivatives, Processes For Their Production And Their Use As Adenosine Receptor Antagonists. EP2465859A1, 2012.

[4] D. F. Evans, J. Chem. Soc. 1959, 2003.

[5] D. F. Evans, G. V. Fazakerley and P. R. F. Phillips, J. Chem. Soc. A, 1971, 1931.

[6] H. D. Crawford and J. Swanson, J. Chem. Educ., 1971, 48, 382.

[7] D. H. Grant, J. Chem. Educ., 1995, 72, 39.

[8] G. J. P. Britovsek, V. C. Gibson, S. K. Spitzmesser, K. P. Tellmann, A. J. P. White and D. J. Williams, J. Chem. Soc. Dalton Trans., 2002, 1159.

[9] SAINT and SADABS, Bruker AXS Inc.: Madison, WI, 2007.

[10] G. M. Sheldrick, SHELXL-97, Program for crystal structure refinement; Göttingen, 1997.

[11] G. M. Sheldrick, Acta Crystallogr., Sect. A: Found. Crystallogr., 2008, 64, 112.

[12] L. J. Farrugia, J. Appl. Cryst., 2012, 45, 849.

[13] Gaussian 16, Revision C.01, Frisch, M. J.; Trucks, G. W.; Schlegel, H. B.; Scuseria, G. E.; Robb, M. A.; Cheeseman, J. R.; Scalmani, G.; Barone, V.; Petersson, G. A.; Nakatsuji, H.; Li, X.; Caricato, M.; Marenich, A. V.; Bloino, J.; Janesko, B. G.; Gomperts, R.; Mennucci, B.; Hratchian, H. P.; Ortiz, J. V.; Izmaylov, A. F.; Sonnenberg, J. L.; Williams-Young, D.; Ding, F.; Lipparini, F.; Egidi, F.; Goings, J.; Peng, B.; Petrone, A.; Henderson, T.; Ranasinghe, D.; Zakrzewski, V. G.; Gao, J.; Rega, N.; Zheng, G.; Liang, W.; Hada, M.; Ehara, M.; Toyota, K.; Fukuda, R.; Hasegawa, J.; Ishida, M.; Nakajima, T.; Honda, Y.; Kitao, O.; Nakai, H.; Vreven, T.; Throssell, K.; Montgomery, J. A., Jr.; Peralta, J. E.; Ogliaro, F.; Bearpark, M. J.; Heyd, J. J.; Brothers, E. N.; Kudin, K. N.; Staroverov, V. N.; Keith, T. A.; Kobayashi, R.; Normand, J.; Raghavachari, K.; Rendell, A. P.; Burant, J. C.; lyengar, S. S.; Tomasi, J.; Cossi, M.; Millam, J. M.; Klene, M.; Adamo, C.; Cammi, R.; Ochterski, J. W.; Martin, R. L.; Morokuma, K.; Farkas, O.; Foresman, J. B.; Fox, D. J. Gaussian, Inc., Wallingford CT, 2016.

[14] A. D. Becke, J. Chem. Phys., 1993, 98, 5648 
[15] S. Grimme, S. Ehrlich and L. Goerigk, J. Comp. Chem., 2011, 32, 1456.

[16] (a) W. J. Hehre, K. Ditchfield and J. A. Pople, J. Chem. Phys., 1972, 56, 2257; (b) P. C. Hariharan and J. A. Pople, Theor. Chim. Acta, 1973, 28, 213; (c) M. M. Francl, W. J. Pietro, W. J. Hehre, J. S. Binkley, M. S. Gordon, D. J. DeFrees, and J. A. Pople, J. Chem. Phys., 1982, 77, 3654. T. Clark, J. Chandrasekhar, G. W. Spitznagel, and P. v. R. Schleyer, J. Comp. Chem., 1983, 4, 294-301.

[17] A. V. Marenich, C. J. Cramer and D. G. Truhlar, J. Phys. Chem. B., 2009, 113, 6378.

[18] T. Lu, F. Chen, Multiwfn: A Multifunctional Wavefunction Analyzer, J. Comput. Chem., 2012, 33, 580-592. 